University of Louisville

ThinkIR: The University of Louisville's Institutional Repository

\title{
The emerging oral pathogen, filifactor alocis, disrupts neutrophil functions to enhance survival and dysregulate inflammation.
}

Irina Miralda Molina

University of Louisville

Follow this and additional works at: https://ir.library.louisville.edu/etd

Part of the Bacterial Infections and Mycoses Commons, and the Oral Biology and Oral Pathology Commons

\section{Recommended Citation}

Miralda Molina, Irina, "The emerging oral pathogen, filifactor alocis, disrupts neutrophil functions to enhance survival and dysregulate inflammation." (2020). Electronic Theses and Dissertations. Paper 3386.

https://doi.org/10.18297/etd/3386

This Doctoral Dissertation is brought to you for free and open access by ThinkIR: The University of Louisville's Institutional Repository. It has been accepted for inclusion in Electronic Theses and Dissertations by an authorized administrator of ThinkIR: The University of Louisville's Institutional Repository. This title appears here courtesy of the author, who has retained all other copyrights. For more information, please contact thinkir@louisville.edu. 
THE EMERGING ORAL PATHOGEN, FILIFACTOR ALOCIS, DISRUPTS NEUTROPHIL FUNCTIONS TO ENHANCE SURVIVAL AND DYSREGULATE INFLAMMATION

\author{
By: \\ Irina Miralda Molina \\ B.S., University of Louisville, 2014 \\ M.S., University of Louisville, 2017
}

\begin{abstract}
A Dissertation
Submitted to the Faculty of the

School of Medicine of the University of Louisville in Partial Fulfillment of the Requirements for the Degree of
\end{abstract}

Doctor of Philosophy in Microbiology and Immunology

Department of Microbiology \& Immunology University of Louisville School of Medicine Louisville, Kentucky

May 2020 
Copyright 2020 by Irina Miralda Molina

All rights reserved 

THE EMERGING ORAL PATHOGEN, FILIFACTOR ALOCIS, DISRUPTS NEUTROPHIL FUNCTIONS TO ENHANCE SURVIVAL AND DYSREGULATE INFLAMMATION

By

Irina Miralda Molina

B.S. University of Louisville, 2014

M.S. University of Louisville, 2017

A Dissertation Approved on

April 15, 2020

By the following Dissertation Committee:

Silvia M. Uriarte, Ph.D.

Matthew B. Lawrenz, Ph.D.

James E. Graham, Ph.D.

Pascale Alard, Ph.D.

Jan Potempa, Ph.D. 


\section{DEDICATION}

This dissertation is dedicated to

my amazing family, Abner ${ }^{2}$ and Carmen ${ }^{2}$ Miralda,

who I try to make proud with every breath I take,

and to those who live defiantly to follow their passion.

"You may say I'm a dreamer, but I'm not the only one," John Lennon. 


\section{ACKNOWLEDGEMENTS}

I would first like to thank my mentor, Dr. Silvia Uriarte for taking a chance on me as an undergraduate student with zero lab experience. In the past 8 years I have come to admire you for your enthusiasm, curiosity, fearlessness, and kindness. Words will never capture my gratitude to you for believing in me, encouraging me, advocating for me, and giving me the confidence to pursue a career in science. We are the perfect example of the quality of student that can evolve from a loving and supportive lab environment and I look forward to spoiling my future students the way you have done with me!

Besides my advisor, I would also like to thank my committee members, Dr. Pascale Alard, Dr. Jan Potempa, Dr. James Graham and Dr. Matthew Lawrenz. Your enthusiasm, support and guidance has been invaluable for the completion of this dissertation. I would specifically like to highlight Dr. Lawrenz, who acted as my department co-mentor. This department and its' students are so fortunate to have you. Thank you for listening, understanding and guiding me in the toughest times of my dissertation. I would also like to give a special thank you to my other co-mentor, Dr. James Graham, who opened his lab for me to earn the microbiology part of this degree. I always cherished your insight and mentorship, and I will always miss our Friday meetings. Thank you to Dr. Russell Salter for offering an avenue to connect with diversity students in STEM. Attending the SACNAS meetings will forever be one of my fondest memories. 
A huge heartfelt thank you to the past and present members of the Uriarte lab family. Chris and Max, thank you for your being amazing lab mates and contributing to my project. To the brilliant Dr. Aruna Vashishta, thank you for everything. Our collaboration and friendship is one of the things I will cherish the most from my time in graduate school. To our close collaborators (and their labs), Dr. Richard Lamont and Dr. Juhi Bagaitkar, without your knowledge, expertise, and this project would not have been possible. Juhi, thank you for always taking time out of your busy schedule to teach me techniques and give me too many ideas for experiments. Your passion for science and learning is infectious, and I look forward to seeing your career skyrocket. I would also like to say a heartfelt thank you to Terri Manning who is an incredible person and has tolerated me being a neutrophil tyrant for the past year. Similarly, I would like to thank all of the amazing past and present members of the Kidney Disease Program, who have helped me grow up as a scientist and as a person.

I cannot begin to express my appreciation to my many friends, who have lent their joy during the successess and their support during the difficult times. It really does take a village, and I'm so glad they were mine. Finally, it is difficult to describe the debt of gratitude I feel for my loving family. Your work ethic, tenacity, and passion inspire me every day to be the best version of myself. May this achievement be the first of many to validate the sacrifices you have made to see me succeed. 


\begin{abstract}
:
THE EMERGING ORAL PATHOGEN, FILIFACTOR ALOCIS, DISRUPTS NEUTROPHIL FUNCTIONS TO ENHANCE SURVIVAL AND DYSREGULATE INFLAMMATION
\end{abstract}

Irina Miralda Molina

April 15, 2020

Periodontitis is an irreversible, chronic inflammatory disease where pathogenic microbial communities accumulate in the gingival crevice. Advances in cultureindependent techniques have facilitated the identification of new bacterial species in periodontal lesions, such as the Gram-positive anaerobe, Filifactor alocis. Neutrophils are a major component of the innate host response, and the outcome of their interaction with F. alocis may be a determinant of oral health. Neutrophil functions typically protect the host against periodontal disease, oral pathogens have adapted to evade or disarm neutrophil microbicidal functions while promoting mechanisms that drive inflammation, which also provides a source of nutrients for growth. This study has two main goals: to determine how F. alocis interferes with microbicidal mechanisms to survive in neutrophils and to examine how $F$. alocis contributes to the chronicity of periodontitis by promoting inflammation.

This dissertation characterizes human neutrophil global gene expression during infection with $F$. alocis (Chapter 2). Challenge of human neutrophils with $F$. alocis resulted in the differential expression of genes involved in multiple neutrophil effector functions 
such as chemotaxis, cytokine and chemokine signaling pathways, and apoptosis. F. alocis challenge also affected the expression of components from the TNF $\alpha$ and MAPK kinase signaling pathways. This resulted in transiently dampened p38 MAPK activation by secondary stimuli TNF $\alpha$. Functionally, the $F$. alocis-mediated inhibition of p38 activation by $\mathrm{TNF} \alpha$ resulted in decreased cytokine production but had no effect on priming of the respiratory burst response, or the delay of apoptosis by TNF $\alpha$. Since this modulatory effect was characteristic of viable $F$. alocis only, this is an $F$. alocis' mechanism to control neutrophils and their functional responses.

I also examined neutrophil functional mechanisms in bone marrow neutrophils from TLR2 ${ }^{-/-}$mice (Chapter 3). I found that compared to WT, TLR2 ${ }^{-/-}$neutrophils were more efficient at killing $F$. alocis, but the increase in killing capacity was not due to a difference in phagocytosis or production of ROS. Instead, it was due to an increase in fusion of myeloperoxidase granules with the $F$. alocis phagosome. Meanwhile, $F$. alocis promotes inflammation by inducing the release of pro-inflammatory cytokines through TLR2 signaling. The TLR2 adaptor protein, MyD88, was also critical for cytokine production, but the events downstream of MyD88 follow non-canonical signaling.

Apoptotic pathways were also showed significant changes in mRNA levels when neutrophils are challenged with $F$. alocis. Neutrophils had prolonged lifespans and higher functional capacity than unchallenged cells (Chapter 4). Despite their viability, F. alocischallenged cells were significantly efferocytosed by macrophages, which developed a proinflammatory profile (Chapter 5). Collectively, this data confirms that $F$. alocis interferes with TLR2 signaling to promote its survival within neutrophils and promotes chronic 
inflammation by extending neutrophil lifespan and delaying the resolution of inflammation. 


\section{TABLE OF CONTENTS}

DEDICATION ............................................................................................... ii

ACKNOWLEDGEMENTS _.................................................................................. iv

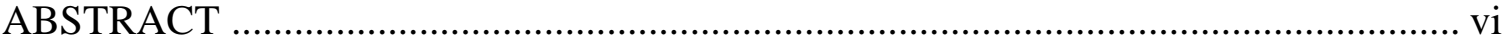

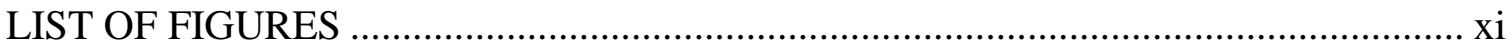

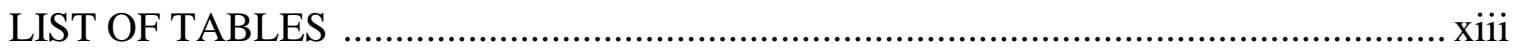

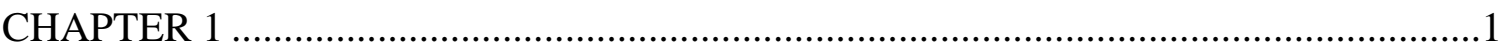

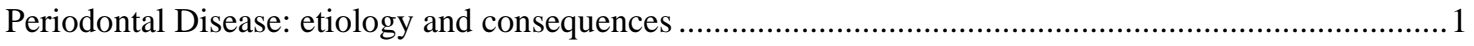

A moving target: Making an inventory of oral microbes in periodontitis ...................................... 4

Filifactor alocis: finding its place in dysbiotic periodontal community .............................................6

Enduring inflammation: F. alocis survival mechanisms \& virulence factors .................................... 8

F. alocis interactions with oral epithelium and monocytes ........................................................

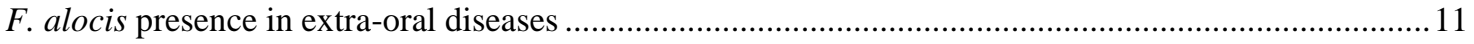

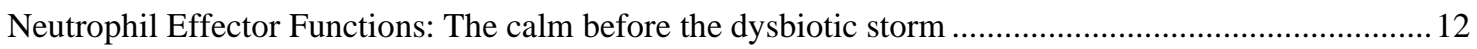

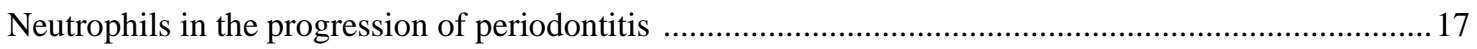

Manipulation of Neutrophil Effector Functions by Periodontal Pathogens ......................................... 19

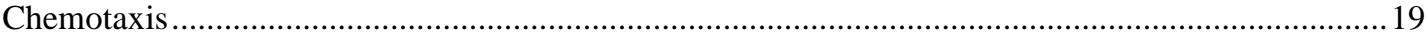

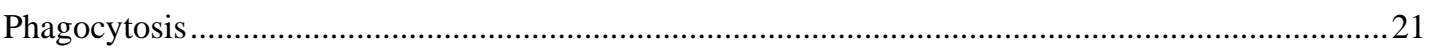

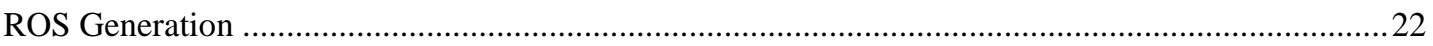

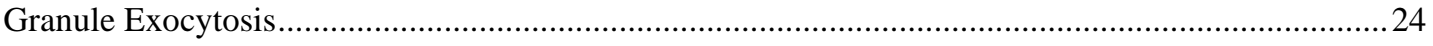

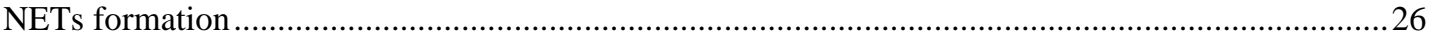

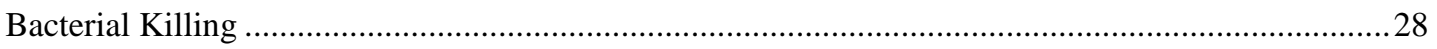

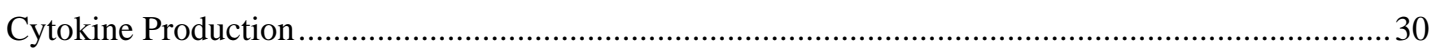

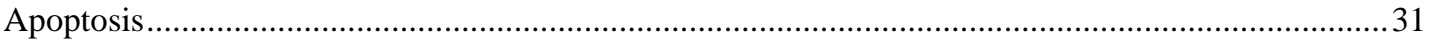

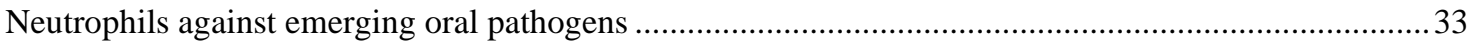

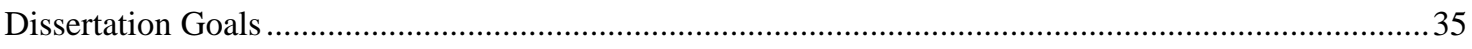

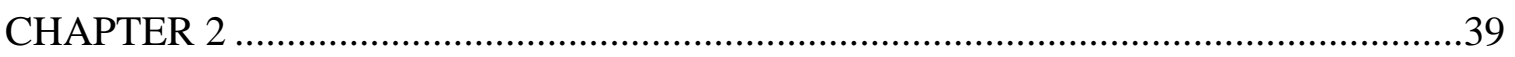

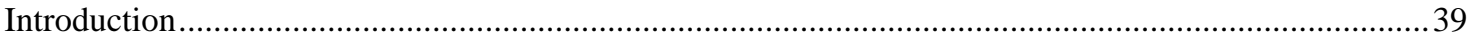

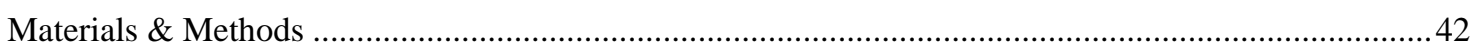

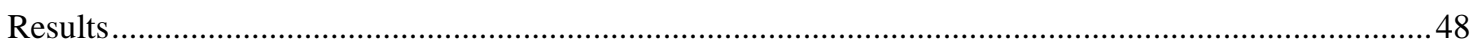

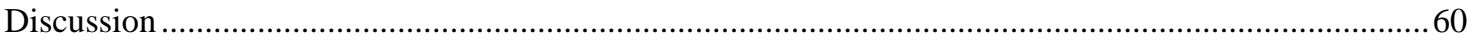




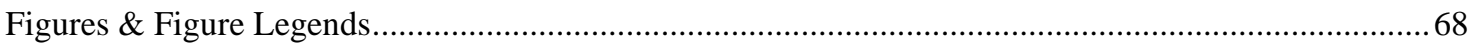

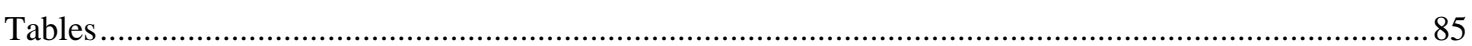

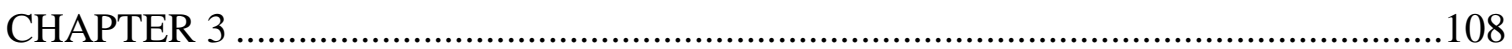

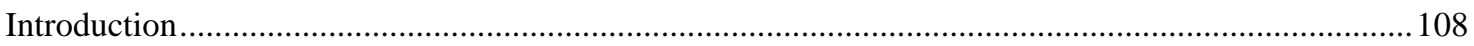

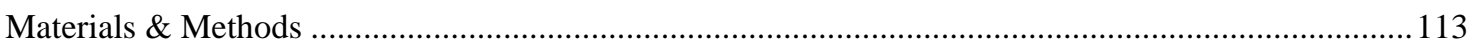

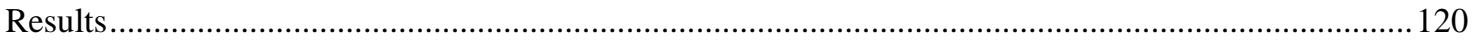

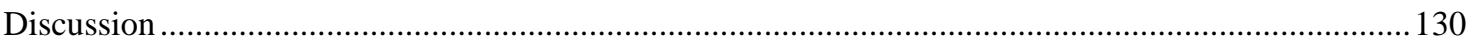

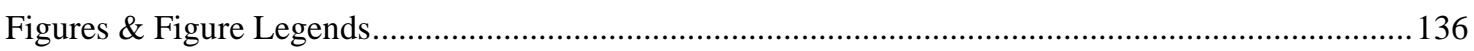

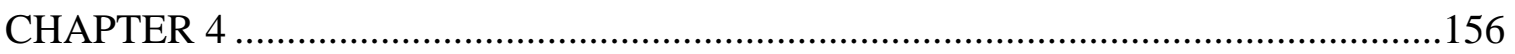

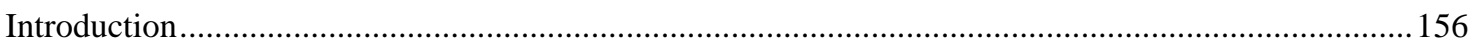

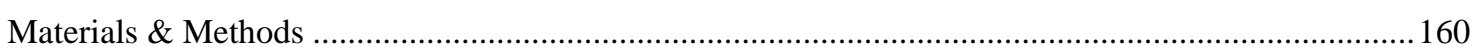

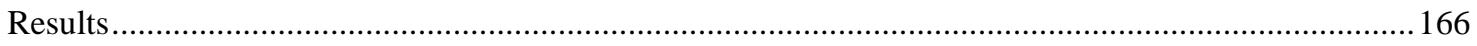

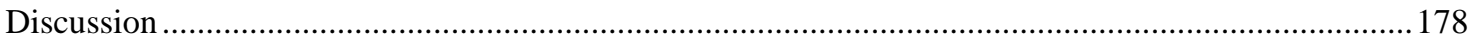

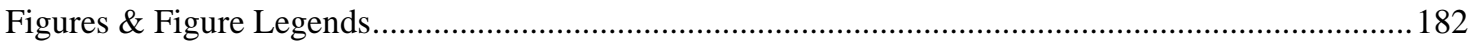

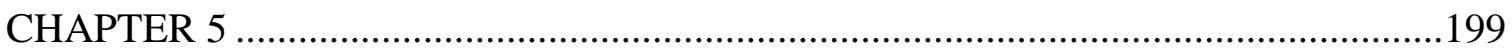

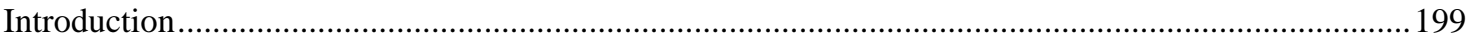

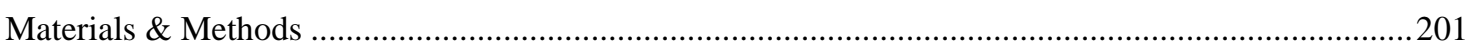

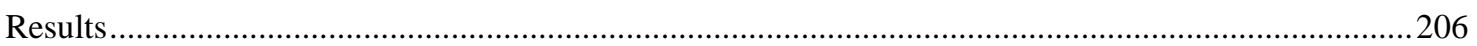

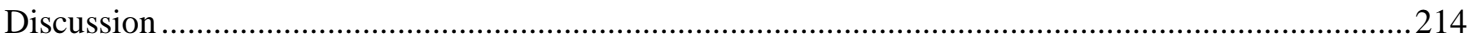

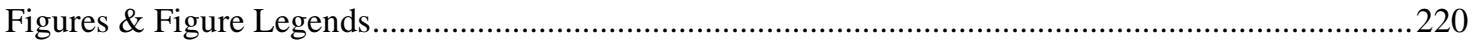

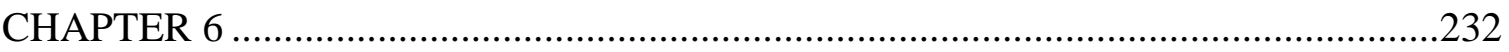

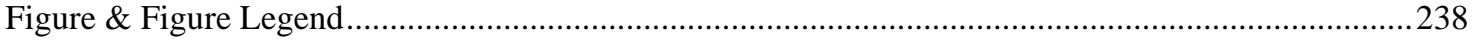

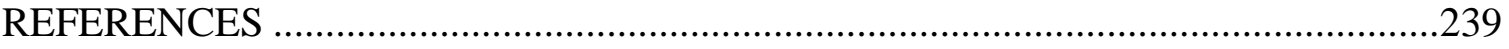

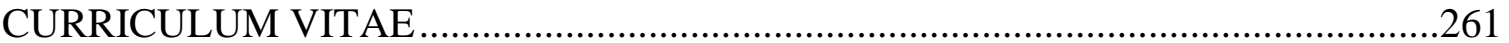




\section{LIST OF FIGURES}

Figure 1.1: Sequential steps of classic neutrophil recruitment and activation.................14

Figure 1.2: Mechanisms by established oral pathogens to disrupt neutrophil functions ...19

Figure 2.1: Global changes in the transcriptome of $F$. alocis-challenged neutrophils ......68

Figure 2.2: Biological processes affected during $F$. alocis challenge. .............................70

Figure 2.3: Pathways affected during $F$. alocis challenge............................................71

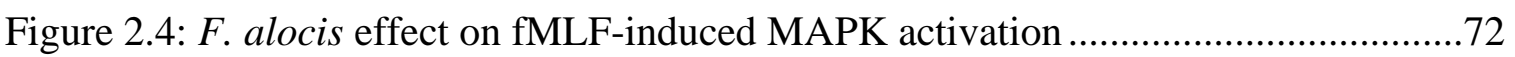

Figure 2.5: F. alocis effect on TNF $\alpha$-induced MAPK activation ....................................74

Figure 2.6: $F$. alocis effect on TNF $\alpha$-induced AKT activation ......................................76

Figure 2.7: Dampened p38 MAPK phosphorylation affects the activation of translation

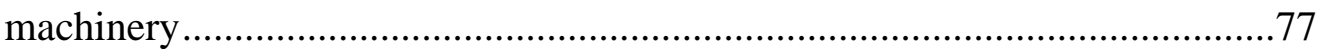

Figure 2.8: F. alocis challenge has no effect on the protein expression of NADPH oxidase

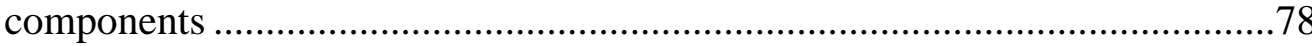

Figure 2.9: $F$. alocis effect on TNF $\alpha$-induced functional responses ...............................79

Figure 2.10: BIRB-796 and 6-hour F. alocis treatment do not inhibit TNF $\alpha$ pro-survival

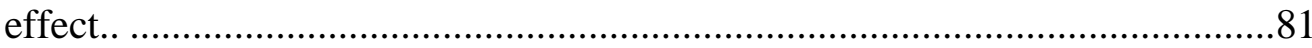

Figure. 2.11: BIRB-796 and 10-hour F. alocis treatments do not inhibit the TNF $\alpha$

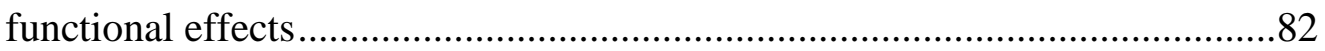

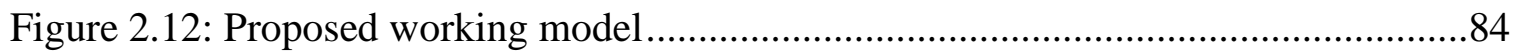

Figure 3.1: Toll-like Receptor (TLR) signaling cascades..........................................110

Figure 3.2: F. alocis induces changes in gene expression of genes in TLR signaling

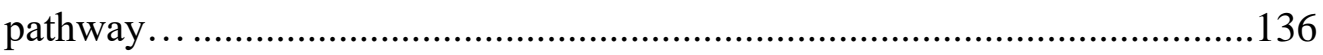

Figure 3.3: F. alocis is recognized by TLR2/6 heterodimers ......................................138

Figure 3.4: F. alocis survival is partially linked to TLR2 signaling..............................140

Figure 3.5: F. alocis induces minimal ROS generation from both WT and TLR2 ${ }^{-/-}$bone

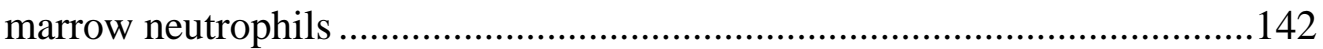

Figure 3.6: F. alocis modulates TLR2 signaling to block phagosome maturation..........143

Figure 3.7: F. alocis activates NF- $\mathrm{BB}$ and induces the production of cytokines through

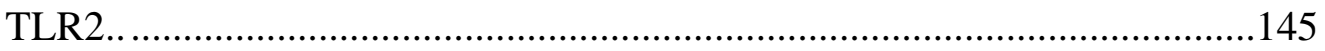

Figure 3.8: MyD88 signaling is not required for $\mathrm{F}$. alocis viability ............................147

Figure 3.9: F. alocis induces minimal ROS generation from both WT and MyD88 ${ }^{-/-}$bone

marrow neutrophils ...................................................................... 149

Figure 3.10: F. alocis phagosome maturation requires MyD88 at long timepoints ........151

Figure 3.11: F. alocis-induced cytokine production is MyD88-dependent.....................153 
Figure 3.12: F. alocis does not affect MyD88 protein levels and does not activate IRAK4

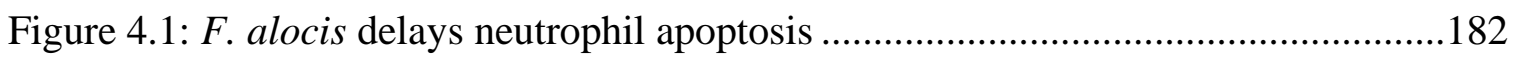

Figure 4.2: F. alocis induces changes in gene expression of apoptotic genes.................184

Figure 4.3: F. alocis induces a pattern of apoptosis gene expression like Anaplasma phagocytophilum ................................................................................186

Figure 4.4: F. alocis upregulates gene expression of anti-apoptotic MCL-1 and delays its

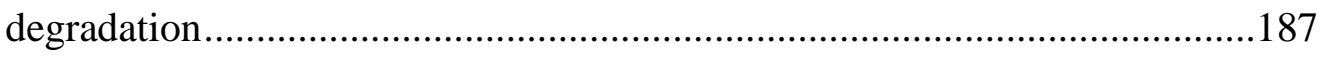

Figure 4.5: F. alocis upregulates genes \& protein expression of XIAP .........................188

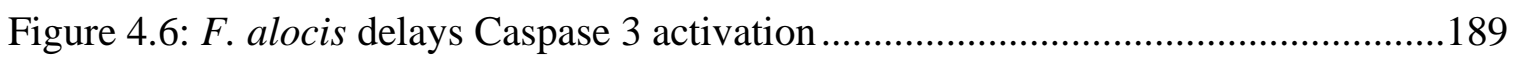

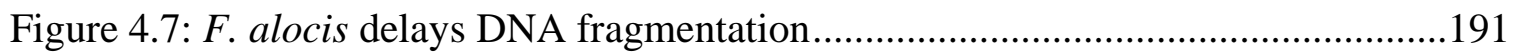

Figure 4.8: F. alocis minimally activates the initiator caspases, 8 and 9 ......................192

Figure 4.9: Contact with $F$. alocis through TLR2/6 is necessary for prolonged neutrophil lifespan......

Figure 4.10: Factors released during F. alocis \& neutrophil interaction are pro-survival 195

Figure 4.11: F. alocis-challenged neutrophils retain functional capacity for longer timepoints

Figure 5.1: Efferocytosis of $F$. alocis-challenged neutrophils is more efficient than media-cultured neutrophils

Figure 5.2: F. alocis-challenged human neutrophils expression of "don't eat me" signals

Figure 5.3: F. alocis-challenged human neutrophils persist in macrophages after 24 hours

Figure 5.4: F. alocis challenged human neutrophils are not efficiently degraded by

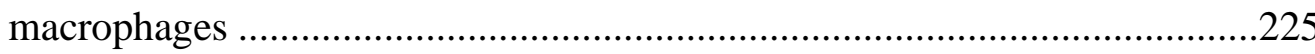

Figure 5.5: F. alocis neutrophils as "trojan horses".

Figure 5.6: Cytokine profile of efferocytic macrophages after ingestion of $F$. alocis neutrophils

Figure 5.7: Chemokine profile of efferocytic macrophages after ingestion of $F$. alocis neutrophils.

Figure 6.1: Working model of $F$. alocis effects on neutrophils. .238 


\section{LIST OF TABLES}

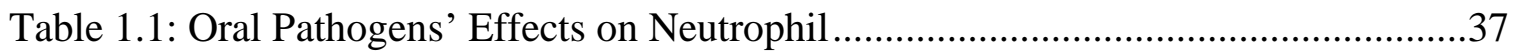

Table 2.1: qPCR primer sequences used to validate RNAseq results.............................85

Table 2.2: Significant biological processes from DAVID analysis categorized by cell

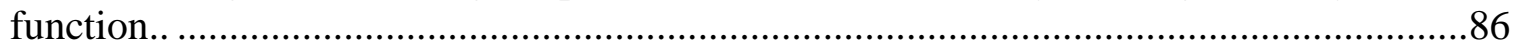

Table 2.3: Genes involved in the Biological Process of Inflammation \& Neutrophil activation by MetaCore

Table 2.4: Fold change of Components of NADPH Oxidase Complex compared to basal.

Table 2.5: KEGG pathways significantly enriched for differentially expressed genes during challenge with $F$. alocis. 


\section{CHAPTER 1:}

\section{INTRODUCTION}

\section{Periodontal Disease: etiology and consequences}

Periodontal diseases affect the integrity of one or several tissues of the periodontium, which is comprised of the gingiva, periodontal ligament, cementum and alveolar bone. The prevalence of the disease is high, affecting $42 \%$ of adults 30 years or older in the USA alone, with older adults and those living under the poverty line being the most susceptible to develop the disease [1]. The term periodontal disease usually refers to the most common inflammatory disorders, gingivitis and periodontitis. Out of the two, gingivitis is the mildest form of periodontal disease and can be easily reversed by effective oral hygiene. On the contrary, periodontitis is an irreversible, chronic inflammatory disease that causes the loss of connective tissue, alveolar bone, and eventually the loss of teeth [2]. Preventative and treatment measures are aimed at controlling or removing the periodontal biofilm and include deep cleaning, antibiotics and surgery in severe cases [3]; but unfortunately, their efficacy is transient since the infection almost always returns. Even in cases where inflammation is resolved and some tissue is regenerated, it is impossible for the lost tooth support to be restored.

The progression of periodontal disease into periodontitis is one of the most severe and irreversible forms of the disease. This development depends on the disruption of 
periodontal host-microbe homeostasis due to a shift in the composition of microbial populations in the oral cavity. Unlike some infectious diseases, periodontitis does not develop from a monoculture infection, but rather the colonization of a heterotypic community of organisms where the presence of one organism enhances the colonization or virulence of others [4]. These interspecies interactions include one organism providing the substrate for attachment and colonization of another, the utilization of released metabolic by-products of one organism as a nutrient source by another organism, or the coordinated break-down of complex substrates [5-8]. The physical interactions among organisms and the diffusion of soluble factors that benefit the whole community results in interdependence between the different bacterial species, each providing a discrete set of communityessential genes. This is explained by the current accepted model for the etiology and pathogenesis of periodontitis, which is called the poly-microbial synergy and dysbiosis (PSD) model $[9,10]$. Under the PSD model, a perturbation of the symbiotic microbial community will result in an increase in the diversity and number of microbial burden. Ultimately, this gives rise to a dysbiotic microbial community that can adapt to and take advantage of the inflammatory environment to enhance the population's bacterial fitness [10].

Under homeostatic conditions, colonizing bacteria initially assemble into physiologically compatible communities that can communicate through sophisticated signaling mechanisms. Any overgrowth or pathology is efficiently controlled by the host inflammatory response, and the gingiva will return to its normal, mild inflammatory state. However, changes in host immune competence, diet, or behaviors like smoking can affect microenvironmental factors like inflammation, $\mathrm{pH}$, redox potential or nutrient availability 
that can drive the selection and enrichment of specific pathogenic bacteria. While the microbiota associated with healthy oral tissues is considered more generalist, the diseaseassociated microbiota is influenced by the traits of specialized microorganisms that have an elevated virulence potential. In mouse models of the disease, the colonization of a keystone pathogen, like Porphyromonas gingivalis, introduces pathogenicity that can elevate the virulence of the entire microbial community, even if there are low numbers of the keystone pathogen present [11]. It is important to note that it is the combination of polymicrobial constituents and their cooperative functional genes that unite to shape and stabilize the dysbiotic microbiota. As the dysbiotic community develops, they will stimulate inflammatory responses that are poorly restrained by susceptible hosts and ineffective at confining the bacterial community. The aggravated and dysregulated responses of the immune system will contribute to tissue destruction that affords a source of nutrients for the microbial community's growth, and thus a continuous cycle of dysbiosis and inflammation ensues.

Importantly, this cycle of dysbiosis and inflammation is not an isolated event. Periodontitis has been linked to systemic inflammation and other adverse impacts on systemic health, amongst them several comorbidities like cancers, rheumatoid arthritis, cardiovascular disease, Alzheimer's disease and other inflammatory conditions [12-15]. The link between these seemingly independent diseases has been explained through several plausible mechanisms. First, locally produced pro-inflammatory cytokines from the oral cavity can enter the bloodstream to induce inflammation in distal sites. For example, tumor necrosis factor (TNF) $\alpha$, interleukin (IL)-1 $\beta$ and IL-6 can cause an acute-phase response in the liver that increases systemic levels of C-reactive protein, fibrinogen and serum amyloid 
A, that contributes to atherosclerosis or intra-uterine inflammation [16, 17]. Through ulceration of the gingiva, periodontal bacteria can also enter the bloodstream and disseminate to other organs. P. gingivalis has been detected in atherosclerotic lesions where it supports a pro-atherogenic environment and Fusobacterium nucleatum has been detected in the placenta where it drives inflammation-induced pregnancy complications $[18,19]$. Finally, since large quantities of bacteria are swallowed daily, it has been proposed that oral bacteria like $P$. gingivalis can alter the gut microbiota and drive increased gut permeability to cause systemic inflammation [20]. Therefore, finding efficient treatments against periodontitis may provide solutions against the exacerbation of many other pathologies.

\section{$\underline{\text { A moving target: Making an inventory of oral microbes in periodontitis }}$}

The microbial shift observed in the gingival crevice from a symbiotic microbiota to a dysbiotic polymicrobial community has been tracked and refined throughout the years as culture-independent techniques have advanced [4, 8, 21, 22]. Initial studies from the turn of the $20^{\text {th }}$ century, used the microbiological techniques available at that time to begin characterizing the etiological agents responsible for periodontitis [23]. Those initial studies discovered spirochetes, fusiforms and streptococci [23, 24] present in periodontal pockets. Later, using microscopic techniques, Listgarten et al. $[25,26]$ showed the frequency of each morphology type in the composition of the subgingival microbiota of patients from different stages of oral disease. The development of immunological techniques and DNA probes helped identify the microbial species present in the subgingival pockets [27]. However, high-throughput analysis of microbial communities from subgingival pockets 
became possible with molecular DNA-based technologies. Using community fingerprinting techniques like restriction fragment length polymorphism [28], or denaturing gradient gel electrophoresis [29], variation and shift in the composition of the microbial community in periodontitis could be identified. The development of DNA-DNA checkerboard hybridization helped to understand the specific association of oral bacteria with health and disease [30]. Socransky et al. [31] described the presence of five microbial communities in subgingival biofilms associated with health and different stages of periodontitis. The 16S rRNA approach has revolutionized the identification of bacterial taxa whether they are cultivable or 'yet-to-be-cultivated', in a mixed population and showed diversity of the oral microbiota [32, 33]. Next generation sequencing technologies further reformed the study of oral microbial diversity with existence of underappreciated periodontal pathogens [34-38]. The pioneer traditional studies together with the more advanced 16S rRNA gene comparative analysis identified presence of approximately 700 predominant taxa in oral microbiome of which approximately $1 / 3$ is 'yet-to-be-cultivated' [39]. About 400-500 taxa were reported in the subgingival crevice alone [32, 37].

Taken together, the plaque analysis from periodontitis-diseased sites has revealed the presence of $P$. gingivalis, Tannerella forsythia and Treponema denticola related with disease severity [31, 40] whereas the presence of Aggregatibacter actinomycetemcomitans has been associated with aggressive periodontitis [24]. F. nucleatum is a commensal species in the oral cavity that increases in abundance in diseased individuals; however, it has been associated with most types of periodontal diseases (chronic and aggressive periodontitis, gingivitis, and endodontic infections) due to its highly synergistic interactions with $P$. gingivalis and $T$. forsythia [41]. The microbes described above are the 
most well-studied periodontal pathogens and have their pathogenic credentials wellestablished. However, examining the microbial composition of the gingival crevice using more advanced techniques revealed the complexity of the microbial communities. A review comparing 41 published studies from 1999 to 2013, showed that 17 newly identified species comprised of five Gram-positive (Eubacterium saphenum, Mogibacterium timidum, Peptoanaerobacter stomatis, Filifactor alocis and Enterococcus faecalis), eight Gram-negative and four not-yet-cultivable group have a moderate association with the etiology of periodontitis [42].

\section{Filifactor alocis: finding its place in dysbiotic periodontal community}

The oral microbiome consists of about 700 predominant taxa [39] and out of these, about 400-500 taxa have been reported in the subgingival cervice alone [32, 37]. Many of these microbial species are "yet-to-be-cultivated," but have been identified due to advances in $16 \mathrm{~S}$ rRNA gene sequencing technology. Filifactor alocis is one of these putative periopathogens identified by culture-independent techniques. Initially, F. alocis was identified from the gingival sulcus in gingivitis and periodontitis patients, and named Fusobacterium alocis [43], but this bacterium was later reclassified under the genus Filifactor [44].

Multiple studies have shown high incidence of $F$. alocis in the subgingival plaques of periodontitis patients, but a complete absence or low number detection in healthy patients [34, 45-47]. A study conducted on 490 subgingival plaques identified $F$. alocis as

the second most prevalent bacteria in chronic periodontitis, the third most prevalent bacteria in generalized aggressive periodontitis, and low prevalence in the control subjects resistant to periodontitis [48]. The occurrence of $F$. alocis has also been reported in 
endodontic infections [49-52] and peri-implantitis [53]. Moreover, a review comparing 41 published studies shows that there is consensus amongst published literature in regards to F. alocis being one of the 17 newly identified species associated with the etiology of periodontitis [42]. Due to the stark difference in colonization of healthy vs. diseased tissues, $F$. alocis has been designated a top marker for generalized aggressive periodontitis along with $P$. gingivalis, $T$. denticola and $T$. forsythia [54].

In the oral cavity, $F$. alocis forms biofilms close to apical and middle thirds of the gingival pocket, which is in close proximity to soft tissue [48]. A comparison of the subgingival microbiota in periodontitis patients showed that $F$. alocis is more prominent in the fourth layer of plaque, which is characterized as a loose layer between the attached biofilm and the soft tissue where some traditional pathogens like T. denticola are also found [55]. When the biofilms and saliva of healthy adults were cultured for three weeks in growth media that simulates the inflamed subgingival environment, there was an enrichment of periodontal pathogens like $P$. gingivalis, $F$. alocis, and several Peptostreptococcus and Treponema spp, which further establishes the classification of $F$. alocis an inflammaphilic oral pathogen [56]. This finding also supports the idea that inflammatory changes in the subgingival nutritional environment favors an increase in the abundance of pathogenic bacterial species like F. alocis.

Characterization of the bacterial composition of different oral sites like saliva, the supragingival area, or the subgingival area showed positive correlation and possible synergistic interactions between $F$. alocis and eight oral pathogens including $P$. gingivalis and T. forsynthia [57]. Further, in vitro studies showed that co-culture of $F$. alocis with $P$. gingivalis enhanced biofilm formation [58] and this biofilm is heterotypic, with enhanced 
$P$. gingivalis growth [59]. Similarly, $F$. alocis can also form biofilms with $F$. nucleatum and A. actinomycetemcomitans [59]. Taken together, these studies demonstrate that $F$. alocis is strongly associated with periodontitis, and that $F$. alocis is a key player in biofilm formation.

\section{Enduring inflammation: $F$. alocis survival mechanisms \& virulence factors}

F. alocis is a slow-growing, non-spore forming, obligate anaerobic rod that has been classified as Gram positive and asaccharolytic, due to its metabolic preference for specific amino acids like arginine $[44,60,61] . \quad F$. alocis manufactures many proteins involved in arginine and citrulline synthesis pathways such as arginine deiminase, aminotransferases, arginine decarboxylase, and acetyl ornithine transferase, suggesting that the nutritional requirement is adequately fulfilled by the byproducts of the disrupted host tissue produced as the consequence of the chronic periodontal inflammation [21, 58, 61]. Also, arginine degradation helps increase the $\mathrm{pH}$, which counteracts the acidic conditions generated through carbohydrate metabolism in the mixed oral community [62].

One of the properties of $F$. alocis that plays a role in the bacterium's survival in periodontal tissues is its resistance to oxidative stress; in fact, its growth was stimulated in media supplemented with hydrogen peroxide [58]. This is likely mediated through $F$. alocis' expression of superoxide reductase, which catalyzes the conversion of toxic superoxides into hydrogen peroxide. Additionally, the presence of sialidase activity helps F. alocis play the role of oxidative sink and stabilize the microbial community in the inflammatory environment of periodontal pockets [62]. F. alocis' contribution enhanced P. gingivalis survival in hydrogen-peroxide-induced oxidative stress [62]. 
Another important asset of $F$. alocis is the presence of different proteases. While most of them are membrane bound, they play an important role in protein or peptide modification and secretion. For example, a collagen peptidase was identified in the $F$. alocis extracellular fraction that can contribute to tissue destruction in periodontitis [63]. F. alocis also expresses a cytoplasmic enzyme, acetyl-ornithine transaminase, that is involved in arginine catabolism but also inhibits the activation of complement component 3 convertase ( $\mathrm{C} 3$ convertase) when present on the bacterial surface. Because of the discovery of this dual function, it was designated as F. alocis complement inhibitor, FACIN [64]. FACIN, lipoproteins, autolysins, and another 28 proteins were detected in the extracellular vesicles from $F$. alocis [65]. Outer membrane vesicles like these represent an efficient mode of disseminating bacterial effector components that manipulate host cell responses. To conclude, all these studies show that $F$. alocis has the arsenal to disrupt normal host cell processes and shields itself and bystander bacteria from the hostile environment of periodontitis.

\section{$\underline{F \text {. alocis interactions with oral epithelium and monocytes }}$}

The inflamed environment of subgingival plaques favors the growth of periodontitis-associated pathogens like F. alocis [56], which is further supported by the finding that this bacteria is abundantly present in subgingival plaques $[34,46]$. Since $F$. alocis is mostly found in the deep layers of biofilm close to the soft tissue, it is constantly interacting with host cells. Studies focusing on the interaction of $F$. alocis with gingival epithelial cells have shown that this bacterium can adhere and invade epithelial cells [62, 66]. Upon contact with $F$. alocis, pro-inflammatory cytokines like TNF- $\alpha$, IL-1 $\beta$ and IL-6 
are secreted from epithelial cells and the bacteria induces apoptosis in oral epithelial cells via activation of caspase 3 and suppression of MEK1/2 signaling pathway [66]. When $F$. alocis is co-cultured with $P$. gingivalis and epithelial cells, the level of internalization of both bacteria by epithelial cells increases more than in monoculture. Their uptake is mediated through endocytic events, and electron microscopy showed that the endocytic vesicles contained both pathogens. Ultimately, uptake of $F$. alocis and $P$. gingivalis leads to change in epithelial cell morphology and cell death [58]. In human oral keratinocytes, F. alocis induced apoptosis in time and dose-dependent manner through the action of the recombinant $F$. alocis peptidase U32 that has collagenase activity and induces the expression of pro-apoptotic genes like Apaf-1, caspase3 and 9 and cytochrome C [67].

F. alocis can also drive inflammation through the secretion of pro-inflammatory cytokines and mediators from the epithelium and infiltrating monocytes. By activating NOD1 in mesothelial cells, heat-killed $F$. alocis induces the expression of CXCL-1, an inflammatory neutrophil chemokine [68]. Additionally, F. alocis causes increases in matrix metalloproteinase (MMP)-1 gene expression and protein synthesis in fibroblastic and monocytic cells that can further contribute to tissue damage in periodontitis [69]. $F$. alocis also induces release of IL-8, IL-6, and TNF- $\alpha$ from human monocytes and human oral epithelial cells in vitro; however, host behaviors can modify this response, since it was reported that marijuana-derived cannabinoids suppress the $F$. alocis-mediated release of pro-inflammatory cytokines by activating the CB2/PI3K axis signaling pathway [70]. Notably, recognition of the whole bacterium is not necessary to elicit pro-inflammatory responses. Extracellular vesicles isolated from the culture of $F$. alocis induced the

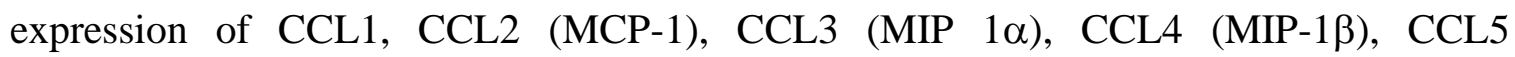


(RANTES), CXCL1, CXCL10, ICAM-1, IL-1 $\beta$, IL-1ra, IL-6, Il-8, Macrophage Migration Inhibitory Factor (MIF), TNF- $\alpha$ and SerpinE in a human monocytic cell line and also caused the expression of CXCL1, granulocyte-colony stimulating factor (G-CSF), granulocyte-macrophage colony stimulating factor (GM-CSF), IL-6 and Il-8 in human oral kerationocytes cell line [65]. Thus, F. alocis and its bacterial products can fuel a proinflammatory environment that benefits the asaccharolytic bacteria's growth.

\section{$\underline{F . \text { alocis presence in extra-oral diseases }}$}

In the past few decades, the link between dental bacteremia and systemic adverse conditions has drawn much attention from researchers [71]. Recent developments in culture-independent techniques has facilitated the detection and identification of microbial species in extra-oral infectious sites [72]. The first in vivo study to address this with $F$. alocis used a mouse subcutaneous chamber model, which showed that $F$. alocis infection (1) triggered a local inflammatory response that included recruitment of neutrophils into the chamber and proinflammatory cytokine production, and (2) can exit the inoculation site and colonize distal organs like the spleen, liver, lung, and kidney where presence of the bacteria caused significant damage to both proximal and distal tubular epithelial cells in the kidney [73]. Corroborating the data from this mouse model, two case reports detected the presence of $F$. alocis in extra-oral diseases. In the first report, a high concentration of F. alocis was detected by 16S DNA analysis in the pleural fluid from a 65-year old patient hospitalized for thoracic empyema [74]. The second study reported that 16S rRNA sequencing identified $F$. alocis in the brain abscess fluid from an 85-year old patient hospitalized for dysarthria and left side paralysis [75]. These studies strengthen F. alocis' 
potential for systemic dissemination and position the bacteria as a pathogen that can colonize and drive inflammation in distal organs outside the oral cavity.

\section{Neutrophil Effector Functions: The calm before the dysbiotic storm}

Polymorphonuclear leukocytes, or neutrophils account for $40 \%$ to $60 \%$ of peripheral blood leukocytes in humans and are the most abundant white blood cell present in the gingival crevice or periodontal pocket $[76,77]$. They play an essential role in the innate immune response, as demonstrated by the development of life-threatening infections or uncontrolled inflammation in individuals with severe neutropenia or genetic disruptions of neutrophil anti-microbial capabilities [78-80]. While it is true that to effectively clear invading organisms, neutrophils must be capable of mounting rapid, vigorous responses to activating stimuli, uncontrolled or prolonged neutrophil activation uses antimicrobial responses to injure normal host cells, leading to pathologic changes to tissues and organs in autoimmune and inflammatory diseases like periodontitis [81]. Consequently, although neutrophil activation is normally tightly regulated, oral pathogens have evolved mechanisms to manipulate neutrophil functions to the bacteria's advantage. To better understand how the dysbiotic microbial community in the oral cavity disarms neutrophils at every step of their lifespan, first it is important to appreciate these effector functions at their optimal response. Thus, here I highlight normal neutrophil functions before discussing how oral pathogens distort them.

Figure 1.1 depicts the different functions that neutrophils perform from circulation to the site of infection, which will be described with more detail in this section. Circulating neutrophils exist in a basal state, characterized by non-adherence, a round morphology, 
minimal transcriptional activity, and a limited capacity to respond to activating stimuli. However, microbial invasion or tissue injury will release pathogen-associated molecular pattern (PAMPs) or damage-associated molecular pattern (DAMPs) molecules that induce sentinel immune cells to release pro-inflammatory cytokines. Those cytokines first modify the adhesion molecules on endothelial cells to facilitate the capture of circulating neutrophils [82]. Additionally, the cytokines from tissue-resident cells will affect the adhesion receptor pattern of neutrophils by inducing the shedding of selectins and the fusion of neutrophil secretory vesicles with the plasma membrane, which leads to increased expression of integrins and other receptors that augment the responsiveness of neutrophils. Transmigration through the endothelial cell layer occurs primarily at endothelial cell junctions and is mediated by a number of adhesion molecules [83, 84]. Next, neutrophils will release proteolytic enzymes like gelatinase to traverse the vascular basement membrane and enter the extravascular space [85]. Once there, neutrophils detect the intensity of chemotactic gradients and move with directionality first towards intermediate chemoattractants (IL-8, leukotriene B4, and platelet activated factor) and then towards end target chemoattractants (bacterial formylated peptides and complement fragments C5a and C3a) $[86,87]$. 


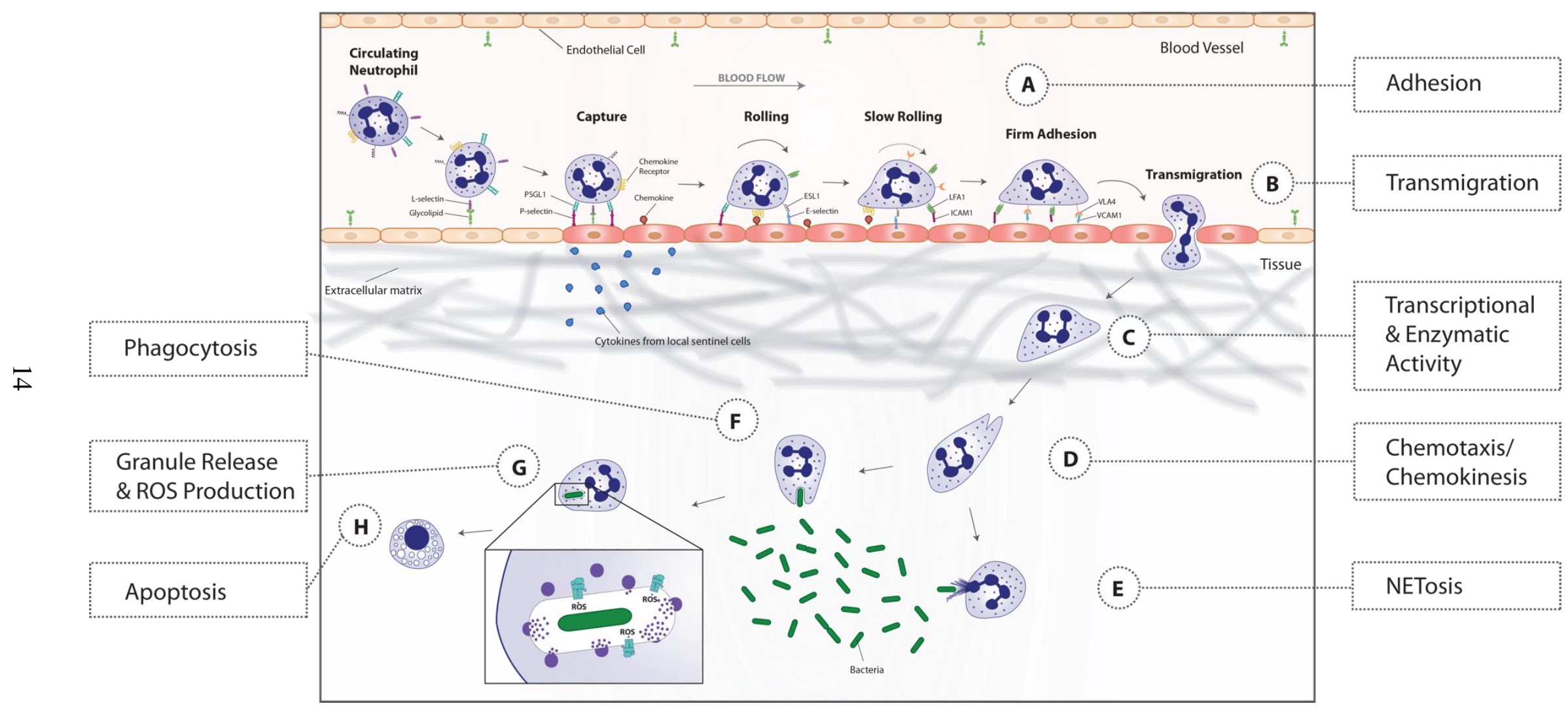

Figure 1.1: Sequential steps of classic neutrophil recruitment and activation. 
As professional phagocytes, neutrophils will rapidly and readily undergo the receptor-mediated process of phagocytosis [88]. Phagocytosis occurs most efficiently by the recognition of complement or IgG-opsonized particles, and while non-opsonized phagocytosis can occur it is less efficient since neutrophils possess fewer non-opsonic receptors than macrophages [89]. Once the particle is engulfed, production of reactive oxygen species (ROS) and fusion of antimicrobial granules will contribute to the phagosome's maturation and lethality [88]. The complementary actions of the granule proteases and ROS production will create a highly toxic environment that few microbes can survive.

Reactive oxygen species (ROS) are generated through the conversion of molecular oxygen to superoxide by the multi-component NADPH oxidase complex. The oxidase is comprised of three membrane subunits (gp91 ${ }^{\text {phox }} / \mathrm{NOX} 2, \mathrm{p} 22^{\mathrm{phox}}$, and Rap1A), and four cytosolic proteins (p47 $7^{\text {phox }}, \mathrm{p} 67^{\text {phox }}, \mathrm{p} 40^{\text {phox }}$, and Rac2). Spatial separation of the membrane and cytosolic components maintains enzymatic inactivity in resting neutrophils. Upon stimulation, the cytosolic components translocate to the membrane bound components to form the catalytically active enzyme complex [90-92]. Notably, neutrophils have the capacity to tailor their response depending on the type of stimuli they encounter. Stimulation of neutrophils by a soluble stimulus, such as fMLF, triggers assembly and activation of the NADPH oxidase at the plasma membrane and release of superoxide anions towards the extracellular space. In contrast, if neutrophils encounter a particulate stimuli, for example a bacterium, assembly and activation of the NADPH oxidase will take place at the membrane of the bacteria-containing phagosome with release of superoxide anions inside the phagosome [93-95]. 
Neutrophil granules are divided into four classes based on granule density and contents [96-99]. Secretory vesicles are created by endocytosis, while gelatinase (tertiary), specific (secondary), and azurophilic (primary) granules are formed from the trans-Golgi network during neutrophil maturation [100]. Like ROS production, the different neutrophil granule subtypes can either be recruited to the bacteria-containing phagosome or stimulated to undergo exocytosis and release their matrix content to the extracellular environment [101]. Normally granule subsets undergo an ordered release based on stimulus intensity, termed graded exocytosis [97, 98]. A weak stimuli induces mobilization of secretory vesicles, and increasing stronger stimulation is required to mobilize gelatinase, specific, and azurophil granules, respectively [102]. The diverse repertoire of proteins and receptors present at the membrane of each granule subtype, as well as within the granule lumen, highlights the important role each granule plays in the different neutrophil responses during inflammation [103-105]. However, the antimicrobial efficacy of neutrophils on intracellular and extracellular microorganisms is enhanced by the ability to combine both oxygen-dependent and independent mechanisms.

Under homeostatic conditions, after their release from the bone marrow into circulation, neutrophils are programmed to undergo apoptosis $\sim 12-24$ hours and are cleared by macrophages in the liver, spleen, and bone marrow [106]. In tissues, neutrophil lifespan is prolonged by cytokines (IL-1 $\beta$, TNF $\alpha$, GM-CSF, G-CSF, and interferon (IFN) $\gamma$ ), microbial components, and the local environment [107]. Moreover intracellular pathogens like Anaplasma phagocytophilum, Chlamydia pneumoniae, and Francisella tularensis can promote neutrophil viability as a way to protect their replicative niche, whereas other microbes like Streptococcus pyogenes, Staphylococcus aureus, and Pseudomonas 
aeruginosa accelerate apoptosis, trigger neutrophil lysis, or redirect cell death towards necrosis to evade intracellular killing [89]. In addition to apoptosis pathways activated through extrinsic receptor ligation or intrinsic mitochondrially-mediated pathways, neutrophils can also undergo apoptosis through a process termed phagocytosis-induced cell death (PICD), that is triggered by phagocytosis of complement- or antibody-coated particles and bacteria, production of NADPH oxidase-derived ROS, and global changes in gene expression $[108,109]$. Apoptosis is a critical step to minimize tissue damage by downregulating the phagocytic and proinflammatory capacity of neutrophils and preventing release of neutrophil cytotoxic components into the extracellular space [110112]. Additionally, timely apoptosis and clearance of neutrophil corpses by macrophages minimizes tissue damage because this process dampens pro-inflammatory cytokine production and reprograms macrophages to a pro-resolution phenotype that favors restoration of tissue homeostasis [113-115].

\section{Neutrophils in the progression of periodontitis}

Neutrophils are the most abundant white blood cell present in the gingival crevice or periodontal pocket [77]. They are recruited into the periodontal pocket by following chemotactic gradients produced endogenously by the junctional gingival epithelium and serum-derived factors or exogenously by the microbial community. This chemotactic gradient is comprised of pro-inflammatory cytokines like interleukin (IL)-8, IL-1b, and chemokines like CXCL12 secreted by gingival epithelial cells, pro-inflammatory serumderived products like C5a, and microbial and biofilm derived chemotactic factors like Nformyl-met-leu-phe (fMLF) peptide, lipopolysaccharide (LPS) [116, 117]. As neutrophils 
migrate toward the crevicular fluid, they will form a protective wall between the bacterial community and the junctional epithelium [118-120]. Here, neutrophil microbicidal mechanisms will reduce the bacterial burden, but the host must strike a balance between the protective and damaging effects of neutrophils since excessive neutrophil-driven inflammation results in collateral tissue destruction.

Neutrophils are indispensable in the innate immune response against bacterial infection and are critical for maintaining homeostasis in the oral cavity (reviewed in [121]). This is best illustrated in patients with neutropenic diseases or genetic defects that affect neutrophil function like Leukocyte adhesion deficiency type I (LAD-I syndrome), Chediak-Higashi syndrome, and Papillon-Lefèvre syndrome, who develop severe periodontitis (reviewed in $[122,123])$. Nonetheless, despite their beneficial role in homeostasis, neutrophils are implicated as the main immune cells responsible for the progression of periodontitis [124, 125]. Under periodontal disease conditions, neutrophils are supernumerary, hyperactivated, or display dysregulated functions. Neutrophil effector functions are likely altered in periodontitis through products from the chronic inflammatory environment that contribute to a primed and hyperactive phenotype [126, 127] or through the manipulating mechanisms of periodontal pathogens, which will be the focus of this section (Table 1.1).

Many studies have compared neutrophils from periodontitis patients to those of healthy controls, and found that while periodontitis neutrophils had augmented phagocytic capacity, they showed defects in chemotaxis, apoptosis and released significantly higher quantities of reactive oxygen species (ROS), anti-bacterial enzymes, neutrophil extracellular traps (NETs), and pro-inflammatory cytokines [128-131]. Together, this 
results in the inefficient killing of microbial pathogens, unresolved inflammation, and the destruction of the tooth supporting tissues via the release of tissue-degrading enzymes or inflammatory and toxic molecules [132-134]. To better understand the interaction of emerging periodontal pathogens like $F$. alocis on neutrophil functions, it is necessary to know the context of how well-established pathogenic oral bacteria can obstruct normal neutrophil functions to promote disease (Figure 1.2).

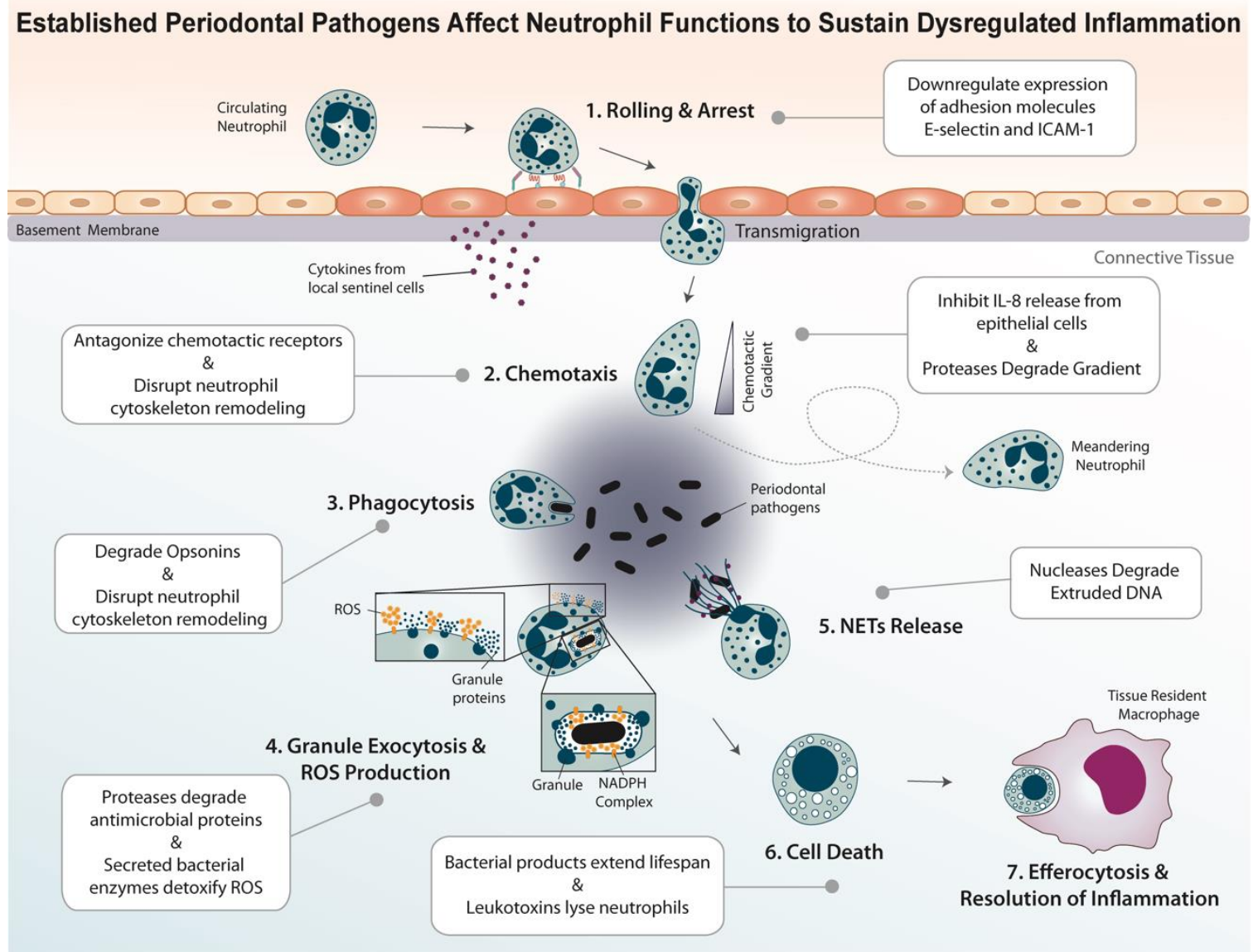

Figure 1.2: Mechanisms by established oral pathogens to disrupt neutrophil functions

\section{Manipulation of Neutrophil Effector Functions by Periodontal Pathogens}

Chemotaxis: 
As the bacterial burden increases during periodontitis progression, neutrophil recruitment and accumulation also magnifies. Not only are neutrophils the predominant immune cell isolated from various oral fluids, but neutrophil counts correlate with the level of inflammation, the increasing depth of the periodontal pocket and the severity of chronic periodontitis $[117,135-137]$. Defects in chemotaxis greatly contribute to the high presence of neutrophils in tissues, and pathogenic oral bacteria are known to modulate this neutrophil function. A clinical study from Roberts et. al showed that peripheral blood neutrophils from chronic periodontitis patients had reduced chemotactic capacity, velocity, and accuracy towards IL-8 and fMLF gradients, which was partially recovered when the patients received non-surgical therapy [138]. One of the most effective ways pathogenic oral bacteria restrict neutrophil chemotaxis is indirectly, by interfering with the chemotactic gradient. $T$. denticola can degrade IL-8 through the action of dentilisin, a major outer membrane protease [139]. Additionally, T. denticola and $P$. gingivalis can both suppress IL-8 production by gingival epithelial cells to cause local chemokine paralysis $[140,141]$.

Alternatively, oral bacteria can disarm neutrophil migration by acting on the neutrophil itself. The supernatant from sonicated organisms like $F$. nucleatum, $A$. actinomycetemcomitans, and $P$. gingivalis can bind and antagonize neutrophil chemotactic receptors to prevent the detection of the chemotactic gradient and thus, inhibit neutrophil migration towards known chemoattractants $[142,143]$. Microbial products like the Major Sheath Protein (MSP) of $T$. denticola can also interfere with cytoskeletal signaling pathways that mediate cell movement [144-148]. Pathogenic oral bacteria also employ misdirection mechanisms: $P$. gingivalis can downregulate the expression of cell adhesion 
molecules like E-selectin and intracellular adhesion molecule (ICAM) 1 that are required for leukocyte extravasation [149-152], while leukotoxin from A. actinomycetemcomitans enhances random migration of neutrophils [153].

When the neutrophil directed migration pattern is altered, they will become vagrant cells and accumulate at the periodontal pocket and adjacent connective tissue. This meandering neutrophil behavior is beneficial to the microbial community because it decreases the chances of neutrophil-bacteria interaction while increasing the chances for bacterial colonization. Paradoxically, the same periodontal bacteria can contribute to the recruitment of neutrophils to the gingival tissue through the actions of their proteases. Mirolysin and karilysin from $T$. forsythia and arginine-specific gingipain (RgpA) from $P$. gingivalis can cleave complement component 5 (C5) to generate the anaphylatoxin $\mathrm{C} 5 \mathrm{a}$, which strongly recruits neutrophils [154-156]. It has been shown that the concentration of gingipains released by $P$. gingivalis is tailored to promote bacterial survival. At early infection stages gingipains can be found at low concentrations, promoting cleavage of C5 and generation of $\mathrm{C5a}$ which stimulates neutrophil recruitment that will enhance tissue destruction and provide nutrients for the dysbiotic community. However, when their concentration increases once the biofilm has been formed in deeper periodontal pockets, they inhibit the complement pathway to protect $P$. gingivalis and bystander bacteria from complement and neutrophil killing [157].

Phagocytosis:

The insidious manipulation of neutrophils by oral pathogens continues at the level of phagocytosis and microbicidal mechanisms. T. denticola and $P$. gingivalis efficiently 
block neutrophil phagocytic events by interfering with cytoskeleton pathways, which provides protective advantages to other bacteria in the oral cavity $[148,158,159]$. Neutrophils treated with MSP from T. denticola had diminished incorporation of actin monomers during de novo filament assembly, which resulted in decreased uptake of IgGcoated spheres [148]. Alternately, P. gingivalis activates PI3K-dependent signaling pathways that suppress RhoA GTPase activity and actin polymerization, thus inhibiting the formation and extension of lamellipodia necessary to form the phagocytic cup [158, 159]. While some oral bacteria like $T$. denticola, A. actinomycetemcomitans, and $P$. gingivalis naturally avoid phagocytosis by neutrophils, the presence of complement and antibodies can overcome this resistance $[128,160,161]$. The problem that arises is that $T$. denticola and $P$. gingivalis proteases can degrade $\mathrm{C} 3$ or other upstream components of the complement pathway to synergistically inhibit complement opsonization and killing [154, 157]. Additionally, despite the finding that patients with periodontitis have high serum IgG levels against $P$. gingivalis, the lysine-specific gingipain $\mathrm{K}(\mathrm{Kgp})$ is able to cleave $\operatorname{IgG} 1$ and $\operatorname{IgG} 3$ at the hinge region, which separates the antigen binding region of the antibody from the effector fragment, resulting in the inactivation of IgG-mediated opsonization [162-164]. When neutrophils are not able to engulf the bacteria, they resort to extracellular killing mechanisms that also drive inflammation.

\section{ROS Generation:}

Activation of neutrophils, either by a particulate or soluble stimulus, results in assembly of the NADPH oxidase multi-component enzymatic complex and robust production of ROS [165]. Generally, ROS produced from neutrophils contributes to 
bacterial killing and intracellular signaling; however, excessive ROS can have cytotoxic effects on periodontal tissues through oxidative damage to DNA and proteins, interference with cell growth and cell cycle progression, and induction of apoptosis of gingival fibroblasts [166-170]. Indirectly, ROS can also induce alveolar bone resorption through their role as intracellular signaling molecules in osteoclastogenic pathways [171]. Like other effector functions, the normal ROS generation by neutrophils is compromised in the context of periodontitis. Neutrophils isolated from subjects with periodontitis released significantly more reactive oxygen species in the absence of stimulus when compared to neutrophils from healthy controls [128], but this was not due to increased expression of NADPH oxidase components [172]. When tested against individual oral bacteria in vitro, neutrophils can mount an ROS response of varying degrees against $T$. denticola, $T$. forsythia, $F$. nucleatum, A. actinomycetemcomitans, and $P$. gingivalis, although the extent of ROS production heavily depends on the strain used, multiplicity of infection, and opsonization [160, 173-177]. Once generated, ROS do not discriminate between host and pathogen cells, and contribute heavily to disease progression since patients with hyperactive ROS response are more susceptible to periodontitis [178, 179]. Notably, patients with Chronic Granulomatous Disease (CGD), who cannot mount a respiratory burst response, are not more susceptible to periodontitis, suggesting that ROS are not critical for maintenance of periodontal health [180].

The contribution of ROS to periodontal destruction was highlighted in a clinical study that treated chronic periodontitis patients with lycopene, an antioxidant [181]. Lycopene-treated patients had reduced oxidative stress and improved clinical parameters like gingival inflammation, periodontal probing depth, and clinical attachment loss. This 
improved outcome could be observed up to 4 months after discontinuing antioxidant treatment, suggesting that if ROS formation can be limited, the destruction of periodontal tissues can also be restricted. The inefficacy of ROS against oral pathogens may be due, in part, to bacterial virulence factors that confer protection for the entire bacterial community. Several oral pathogens like A. actinomycetemcomitans, $F$. nucleatum, and $P$. gingivalis express superoxide dismutase (SOD), which catalyzes the dismutation of superoxides into hydrogen peroxide [182-185]. The master neutrophil manipulator, $P$. gingivalis also expresses rubrerythrin (Rbr) and alkyl hydroperoxidase reductase (Ahp), which detoxify hydrogen peroxide [186-188]. Additionally, through the proteolytic action of gingipains, P. gingivalis can acquire heme deposits on its cell surface, which act as an oxidative sink to further protect bacteria against the deleterious effects of ROS [189, 190]. Other periodontal bacteria may be acting directly on neutrophils to control the ROS response, for example $F$. nucleatum is able to inhibit fMLF-induced superoxide generation, although the mechanism for this phenotype is still unknown [177].

\section{Granule Exocytosis:}

Cationic antimicrobial peptides are one of the very first lines of defense against microbial invaders. In the oral cavity and gingival tissues, the extracellular defense is predominantly mediated by release of human beta-defensins from epithelial cells and neutrophil derived cathelicidin-type peptide LL-37 and alpha-defensins found in the neutrophil cytoplasm and granules [191]. The crevicular fluid from chronic periodontitis patients contained significantly elevated quantities of LL-37 and alpha-defensins [192, 193]; regardless, periodontal pathogens have increased resistance to antimicrobial 
peptides, though this response seems to depend on the strain of bacteria tested [194-198]. The high resistance of periodontal pathogens to bactericidal activity of these peptides can be partially explained through the action of bacterially derived proteolytic peptides. From T. forsythia, miropin, and karilysin efficiently inhibited the activity of a broad range of proteases (LL-37, neutrophil and pancreatic elastases, cathepsin G, subtilisin, and trypsin) $[199,200]$, and gingipains from $P$. gingivalis directly cleaved multiple host antimicrobial proteins, which conferred resistance against extracellular, non-oxidative killing mechanisms [201-204]. Interestingly, the expression of these gingipains is increased when $P$. gingivalis undergoes oxidative stress, suggesting there is a synergistic, compounding attack against neutrophil mechanisms [205].

While neutrophil granule contents can be released into the extracellular matrix or into a microbe-containing phagosome, most studies related to periodontal pathogens have focused on the release of neutrophil granule contents in the context of tissue degradation. A. actinomycetemcomitans, $F$. nucleatum, $T$. denticola, and $P$. gingivalis have all been reported to induce the release of Matrix metalloproteinase 8 (MMP8), Matrix metalloproteinase 9 (MMP9), elastase and lysozyme [173, 196, 197, 206-209]. The exocytosis of neutrophil granules has also been confirmed in neutrophils isolated from the oral cavity, since neutrophils from periodontitis patients express increased levels of degranulation markers on their surface [210]. Elevated levels of neutrophil elastase, plasminogen, and MMP9 were detected in periodontal ligament from chronic periodontitis patients [211]. Zymographic analysis of these three proteinases was tested in vitro on periodontal ligaments, and elastase was the only proteinase involved in the degradation of collagen fibrils. Notably, the morphological features from the in vitro system were similar 
to that of the periodontal ligament in chronic periodontitis, which directly implicates this neutrophil enzyme in the early destructive stages of periodontal disease [211]. Similarly, bacterial proteases can also directly induce tissue degradation. Karilysin from T. forsythia shares structural homology to human matrix metalloproteinases and can cleave elastin, fibrinogen and fibronectin [212].

Host-derived protease inhibitors are produced locally as negative-feedback loops to maintain homeostasis in oral tissues and regulate the activity of neutrophil proteases [213]. Two of these protease inhibitors are secretory leukocyte protease inhibitor (SLPI) inhibits neutrophil elastase activity and elafin targets elastase and protease 3 (PR3). Gene expression of these protease inhibitors was significantly induced when gingival epithelial cells were infected with $P$. gingivalis, but not $T$. forsythia, A. actinomycetemcomitans, or F. nucleatum [214]. It has been suggested that $P$. gingivalis manipulates this system to specifically dampen neutrophil antimicrobial responses since SLPI and elafin have no inhibitory action against gingipains [215]. Paradoxically, gingipains will degrade SLPI and elafin, supporting the notion that $P$. gingivalis sustains some level of inflammation to maintain infection $[214,216]$. Taken together, these studies show there is an additional level of complexity where oral pathogens can interfere with the host regulation of extracellular neutrophil granule protein activity to protect the microbial community while still maintaining inflammation.

\section{NET formation:}

NETs represent an immune defense mechanism deployed by neutrophils to immobilize and kill invading microbes or contain biofilms from disseminating into other 
sites of the body [217]. However, as with all other neutrophil mechanisms, this function is a double edged sword that has also been implicated in inflammation and induction of autoimmunity [218]. Confocal and electron microscopy of gingival biopsies and crevicular exudates from patients with chronic periodontitis confirmed there was NET formation in the oral cavity [219]. A later study found that neutrophils are attracted to the supragingival biofilms, where they are stimulated to release NETs [220]. When individual oral bacteria were tested against neutrophils, A. actinomycetemcomitans and $F$. nucleatum both induced NET formation. F. nucleatum activated neutrophils through nucleotide oligomerization domain (NOD) 1 and 2 and induced a time-dependent, robust release of NETs [221]. This NETosis was independent of TLR stimulation and ROS production [176]. A. actinomycetemcomitans was also able to induce NETosis through stimulation by the whole bacterium as well as through stimulation with leukotoxin alone [153]. The presence of serum and signaling through complement receptor (CR)1 enhanced NET release induced by A. actinomycetemcomitans [222]. Contrasting reports have shown that $P$. gingivalis does $[223,224]$ and does not [176] induce NET formation, though this conflicting data may be mediated by bacterial strain differences. No studies have directly tested whether $T$. forsythia or T. denticola can induce NET release from neutrophils. Regardless of whether NETosis was induced by the oral pathogen, both A. actinomycetemcomitans and $P$. gingivalis could be trapped by HOCl-produced NETs [176].

Despite initial trapping by NETs, several oral microbes like $P$. gingivalis, Prevotella intermedia, and F. nucleatum, but not A. actinomycetemcomitans, express nucleases with differing DNA degradation capacities [225, 226]. Additionally, $P$. gingivalis expresses Porphyromonas peptidylarginine deiminase (PPAD), an enzyme that 
citrullinates histone $\mathrm{H} 3$, thereby facilitating the bacterial escape from NETs [227-230]. When neutrophils were cultured with $P$. gingivalis or purified Rgp gingipains, both stimulants induced NETs that not only lacked bactericidal activity but instead stimulated the growth of bacteria species otherwise susceptible to killing in NETs [224]. The protection against killing by NETs was mediated through the proteolysis of bactericidal components on the NETs. Taken together, some periodontal pathogens may be playing a dual role in NETosis: they are the potent direct inducers of NETs formation but simultaneously prevent bacterial entrapment and subsequent killing by NETs.

\section{Bacterial Killing:}

Neutrophils isolated from the crevicular fluid of periodontitis patients showed decreased phagocytic activity and intracellular killing against A. actinomycetemcomitans and $P$. gingivalis as compared to neutrophils from healthy controls, indicating that there is a pervasive inhibition of bacterial killing during periodontitis [231]. When cultured under anaerobic conditions, neutrophils were unable to eliminate $P$. gingivalis as tested by colony forming units (CFU) [204]. P. gingivalis was also resistant to killing by neutrophil granule contents, likely because $P$. gingivalis culture supernatant contains ginigipains that could inactivate cathepsin $\mathrm{G}$, elastase, bacterial-permeability increasing factor and defensins [204, 232]. Addition of gingipain inhibitors to the P. gingivalis culture supernatant and neutrophils, neutrophils regained their microbicidal capacity against the bacteria [233]. Neutrophils were also able to significantly kill $F$. nucleatum and $A$. actinomycetemcomitans strain $\mathrm{Y} 4$ within an hour $[128,234,235]$. Other strains of $A$. actinomycetemcomitans were able to resist intracellular microbidical mechanisms after an 
hour of challenge, which correlates with the levels of leukotoxin expression [236]. Notably, exposure of neutrophils to nicotine severely dampens their ability to kill $F$. nucleatum and A. actinomycetemcomitans, hinting at the severity of effects from host-driven susceptibility [237]. Killing of T. denticola and T. forsythia has been reported by in vitro studies using macrophages [238, 239], but to the best of our knowledge, no studies have examined whether neutrophils can directly kill $T$. denticola or T. forsythia.

Whether the pathogenic bacteria can block killing by neutrophils on its own is not as important in the case of the periodontal dysbiotic community because the protective effects from one species could benefit others that do not have any virulence factors of this type. Succinic acid, a metabolic, fatty acid byproduct of $P$. gingivalis and other Bacteroides species, abolished neutrophil killing of Escherichia coli by decreasing neutrophils ability to produce ROS [240, 241]. Similarly, when neutrophils were incubated with short chain fatty acids derived from anaerobic bacteria, their ability to undergo granule exocytosis and produce ROS was also diminished [242].

Out of the established periodontal pathogens, P. gingivalis' survival around neutrophils is the best characterized and depends on the cross-talk between two receptors on the surface of neutrophils in vivo $[158,243]$. While $P$. gingivalis directly activates TLR2/1 receptors, the C5a formed by the gingipain-dependent cleavage of C5 will activate the C5a receptor (C5aR1). The co-stimulation of these receptors results in the degradation of the TLR adaptor protein MyD88 [158, 244]. This reroutes signaling through another adaptor protein, MyD88 adaptor-like (Mal, also known as TIRAP). This is significant because activation of the MyD88-dependent signaling pathway is associated with the initiation of antimicrobial responses while Mal-dependent pathways activate PI3K and 
block phagocytosis, while still resulting in the release of pro-inflammatory cytokines. Thus, by redirecting signaling through Mal instead of MyD88, $P$. gingivalis dismantles the killing activity of neutrophil, but not the proinflammatory activity [122]. Nonetheless, little to no literature is available to explain how some oral pathogens can survive within the hostile environment of the neutrophil phagosome.

\section{Cytokine Production:}

The role of neutrophils as regulators of the immune response has gained increasing recognition because of their capacity to transcribe, perform de novo synthesis, and release different cytokines and chemokines [245-247]. Depending on the type of stimulation they encounter, neutrophils can produce and release an array of different cytokines and chemokines, which is significant in the amplification loop of the local immune response [246-250]. Although each individual neutrophil may not produce quantities comparable to other immune cells, in periodontitis where neutrophils accumulate in great numbers, the collective release of cytokines and chemokines by neutrophils can especially play a role in tissue damage. It is unknown whether T. forsythia or A. actinomycetemcomitans induce direct cytokine production by neutrophils, but reports show that T. denticola, F. nucleatum, and heat-killed $P$. gingivalis induce release of pro-inflammatory cytokine IL-1 $\beta[160,251]$. F. nucleatum and its LPS also induce robust release of IL-8 and TNF $\alpha$ from neutrophils $[131,177]$. LPS from A. actinomycetemcomitans stimulated the release of significantly greater amounts of IL- $1 \beta, \mathrm{TNF} \alpha$, and IL- 8 than the response elicited by $P$. gingivalis-LPS [252]. However, challenge of neutrophils with $P$. gingivalis results in the release of TNF $\alpha$, IL-8, and CCL2 $[252,253]$. A noteworthy distinction is that the best practice for measuring 
neutrophil-derived cytokines is to test a neutrophil population that is as pure as possible since contamination of even $0.01 \%$ of monocytes can skew the cytokine and chemokine populations significantly $[254,255]$.

Apoptosis:

Signs of cell death are readily observed in gingival tissue [256, 257]. The gingival tissues of chronic periodontitis patients show increased activity of caspase 3 and caspase 7, the executioners of apoptotic cell death [258]. Moreover, periodontal pathogens, such as T. denticola, $P$. gingivalis, and $T$. forsythia, elicit the release of adenosine triphosphate (ATP), uric acid, heat shock protein (HSP)60, and High Mobility Group Box (HMGB)1 from THP-1 derived macrophages, indicating the presence of pyroptotic cell death in periodontal tissue [259]. Although cell death pathways in epithelial cells and macrophages have been well studied after interaction with periodontal pathogens, there are only a few publications that have addressed neutrophil viability after encounters with oral bacteria.

Transcriptionally, neutrophils from chronic periodontitis patients have significantly upregulated pro-survival pathways [260]; however, most studies on periodontal bacteria and neutrophil lifespan have only measured cell death at very short-timepoints (three hours or less) post bacterial challenge. For example, $F$. nucleatum, $P$. gingivalis and $T$. denticola did not induce lactate dehydrogenase $(\mathrm{LDH})$ release from neutrophils after 1-hour challenge with neutrophils [196]. Incubation of neutrophils with T. denticola for an hour resulted in a mild increase in cell death only detected in neutrophils that phagocytosed antibody coated $T$. denticola at a multiplicity of infection (MOI) of 100 bacteria per neutrophil [160]. Under the same experimental set-up, F. nucleatum induced cell death in 
a MOI and antibody opsonization-dependent manner $[160,261]$. The induction of apoptosis persisted when neutrophils were treated with multiple strains of $F$. nucleatum [177]. Uptake of antibody-opsonized A. actinomycetemcomitans after 1 hour of challenge also resulted in the rapid cell death of neutrophils [236]. The induction of apoptosis after such short timepoints suggests the neutrophils challenged with A. actinomycetemcomitans, $P$. gingivalis, $F$. nucleatum, and $T$. denticola underwent phagocytosis-induced cell death (PICD), which is linked to the production of ROS [108, 262]; however, since periodontal bacteria can resist neutrophil phagocytosis, the probability of PICD may not occur very often in vivo.

Compounding the length of neutrophil lifespan is the presence of several proinflammatory mediators that promote neutrophil longevity at sites of inflammation, such as LPS, liopoteichoic acids (LTA), TNF $\alpha, \mathrm{C} 5 \mathrm{a}, \mathrm{IL}-1 \alpha$, granulocyte-macrophage colonystimulating factor (GM-CSF), granulocyte colony-stimulating factor (G-CSF), and interferon (IFN) $\gamma$, and render these granulocytes resistant to extrinsic ligand-induced apoptosis (FS-7-associated surface antigen (FAS) and TNF) [263, 264]. Additionally, the products of periodontal bacteria can also lead to changes in neutrophil survival. Neutrophil challenge for 1-3 hours with MSP from T. denticola does not induce cell death [148] and culture of neutrophils with the LPS from three different $P$. gingivalis strains delayed apoptosis in a dose-dependent fashion [265-267], but leukotoxin from $A$. actinomycetemcomitans directly lyses neutrophils [236, 268]. Notably, it is unknown whether direct interaction with $T$. forsythia or products from the bacteria will affect neutrophil lifespan. 
Induction of neutrophil apoptosis is beneficial to the bacteria because it will prevent neutrophil phagocytosis and microbicidal mechanisms from eliminating the bacterial burden. However, when neutrophil apoptosis is delayed, the inflammophillic bacteria still profit because inflammation worsens by two mechanisms: (1) collateral tissue damage increases because neutrophils will accumulate and continue to release degradative neutrophil enzymes and ROS, and (2) resolution of inflammation is delayed because clearance of apoptotic cells though efferocytosis cannot take place, which is essential for the initiation of tissue restoration mechanisms.

\section{Neutrophils against emerging oral pathogens}

Considering the identification of novel bacteria associated with periodontitis, there is still a significant gap in our understanding of the role of these organisms in the disease etiology. Without a full understanding of the pathogenic potential of abundant organisms in the periodontal lesions, there is a substantial barrier to the development of new approaches to prevent or ameliorate the disease. Since neutrophils are a key component of host innate immunity that must be overcome by pathogenic periodontal bacteria, studies on how neutrophils control or are thwarted by emerging oral pathogens afford vital contributions to the scientific community.

So far, characterization of $F$. alocis' interaction with neutrophils has demonstrated that this putative oral pathogen can modulate neutrophil effector functions for its nefarious purposes. Unlike many periodontal pathogens, $F$. alocis does not resist phagocytosis by human neutrophils [269]. In fact, most neutrophils challenged with $F$. alocis rapidly internalized the bacteria independent of viability or opsonization with complement. This is 
likely because viable $F$. alocis is capable of disrupting the neutrophil antimicrobial response. After 4 hours of challenge, $>50 \%$ of $F$. alocis remain viable inside neutrophils, and electron microscopy analysis of $F$. alocis-infected neutrophils showed that electron dense bacteria could still be found inside neutrophil phagosomes after 20 hours of coculture, suggesting a defect in the degradation and clearance of engulfed bacteria.

To dissect how $F$. alocis could actively remain viable despite the toxic capacity of neutrophils, the progression of phagosome maturation was assessed by testing oxygen dependent and independent mechanisms of intracellular killing against viable and heatkilled $F$. alocis. When the production of intracellular ROS was measured, heat-killed $F$. alocis induced a robust ROS response that was four times greater than the amount of ROS produced by neutrophils stimulated with viable $F$. alocis [269]. Furthermore, viable $F$. alocis did not suppress the respiratory burst induced by a secondary stimulus like heatkilled Staphylococcus aureus; suggesting that inhibition of ROS production is not a global effect, but rather a local phagosomal mechanism geared towards promoting survival. The second, oxygen-independent arm of intracellular killing is mediated through the fusion of pre-formed granules with the bacteria-containing phagosome. However, live F. alocis could also control granule trafficking to the phagosome to prevent phagosome maturation. Viable $F$. alocis phagosomes had decreased co-localization with key antimicrobial proteins lactoferrin and elastase whereas twice as many heat-killed F. alocis phagosomes had efficient co-localization [269]. Thus, F. alocis actively subverts elimination by neutrophil by inducing a minimal respiratory burst response and preventing phagosome maturation.

As an inflammophilic bacteria, F. alocis still drives pro-inflammatory processes to secure a source of nutrients for replication. F. alocis is recognized by neutrophils through 
ligation of TLR2, which triggers the exocytosis of three of the four neutrophil granule subtypes through activation of both p38 MAPK and ERK1/2 [270]. The release of toxic granules has implications for tissue degradation, which could be directly observed through gelatin zymography of supernatants of $F$. alocis-stimulated neutrophils [269]. In addition, neutrophils challenged with either live or heat-killed $F$. alocis, showed enhanced random and directed migration towards IL-8; an effect that was dependent on the bacteria-induced granule exocytosis [270].

Remarkably, $F$. alocis appears to play the role of referee and helps limit the system's overall inflammation. Specifically, $F$. alocis does not induce NET formation at any bacterial dose or time tested; additionally, pre-treatment of neutrophils with F. alocis significantly inhibits PMA-induced NETs [271]. F. alocis is recognized by TLR2/6 heterodimers and promotes the release of neutrophil-derived cytokines and chemokines but to a lesser extent compared to another emerging oral bacterium, Peptoanaerobacter stomatis [253]. Additionally, F. alocis induced significant release of anti-inflammatory cytokine, IL-1ra, which was greater than the amount induced by $P$. gingivalis.

\section{$\underline{\text { Dissertation Goals }}$}

Much work remains to fully understand the mechanism of how $F$. alocis contributes to the progression of periodontitis. A large piece of that puzzle is to define how the putative oral pathogen interacts with neutrophils. Based on published work, $F$. alocis follows a similar pattern as described for other periodontal pathogens, to evade killing by neutrophils while simultaneously promoting inflammation. Nonetheless, it was unknown how F. alocis manipulates neutrophil functions. I hypothesized that $F$. alocis interferes with signaling 
pathways to block extermination by neutrophils while inducing a pro-inflammatory environment, so I completed a transcriptome study of $F$. alocis-challenged neutrophils (Chapter 2) and found that Toll-like receptor (TLR) signaling (Chapter 3), apoptosis pathways (Chapter 4), and efferocytic clearing (Chapter 5) are affected in F. alocischallenged neutrophils. 
Table 1.1: Oral Pathogens' Effects on Neutrophil

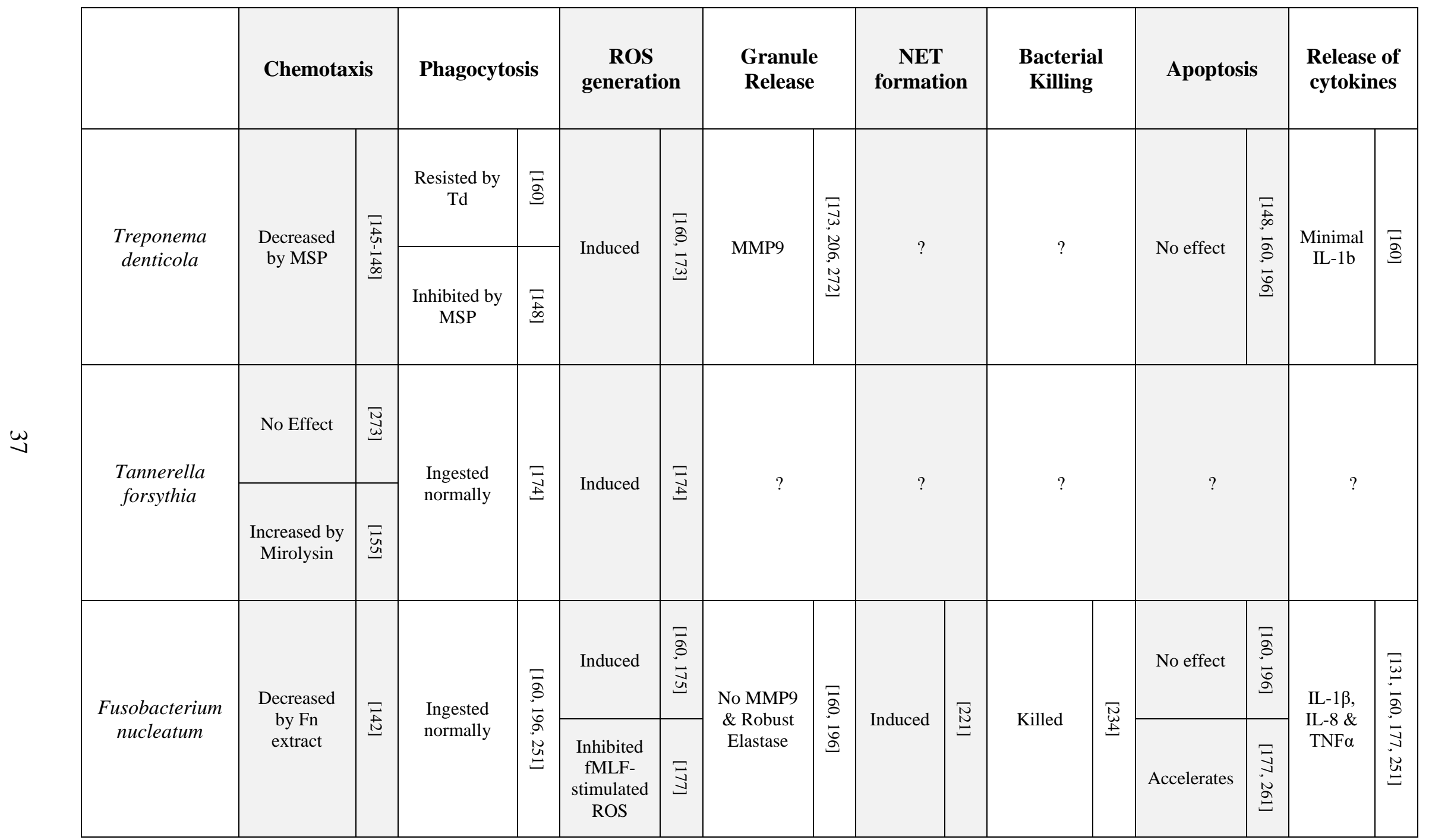




\begin{tabular}{|c|c|c|c|c|c|c|c|c|c|c|c|c|c|c|c|c|}
\hline \multirow{3}{*}{$\begin{array}{l}\text { Aggregatibacter } \\
\text { actinomycetem- } \\
\text { comitans }\end{array}$} & \multicolumn{2}{|c|}{ Chemotaxis } & \multicolumn{2}{|c|}{ Phagocytosis } & \multicolumn{2}{|c|}{$\begin{array}{c}\text { ROS } \\
\text { generation }\end{array}$} & \multicolumn{2}{|l|}{$\begin{array}{l}\text { Granule } \\
\text { Release }\end{array}$} & \multicolumn{2}{|c|}{$\begin{array}{c}\text { NET } \\
\text { formation }\end{array}$} & \multicolumn{2}{|c|}{$\begin{array}{c}\text { Bacterial } \\
\text { Killing }\end{array}$} & \multicolumn{2}{|c|}{ Apoptosis } & \multicolumn{2}{|c|}{$\begin{array}{l}\text { Release of } \\
\text { cytokines }\end{array}$} \\
\hline & $\begin{array}{l}\text { Decreased } \\
\text { by Aa } \\
\text { extract }\end{array}$ & 䓌 & Resists & $\pi$ & & $=$ & $\begin{array}{l}\text { MMP8 by } \\
\text { Aa \& }\end{array}$ & N & & $\overline{\bar{u}}$ & Not Killed & 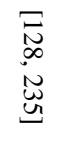 & $\begin{array}{c}\text { Accelerated } \\
\text { by Aa }\end{array}$ & $\begin{array}{l}\bar{N} \\
\text { G } \\
0\end{array}$ & $\begin{array}{l}\text { IL-1 } \beta \text {, } \\
\text { TNF } \alpha \\
\text { and IL- }\end{array}$ & $\pi$ \\
\hline & $\begin{array}{c}\text { Increased by } \\
\text { leukotoxin }\end{array}$ & 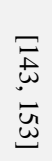 & phagocytosis & 9 & & $u$ & $\begin{array}{l}\text { Elastase by } \\
\text { Leukotoxin }\end{array}$ & $\tilde{\infty}$ & & 孞 & Killed & 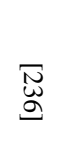 & $\begin{array}{l}\text { Lysed by } \\
\text { leukotoxin }\end{array}$ & $\begin{array}{l}\bar{N} \\
\mathscr{\alpha}\end{array}$ & $\begin{array}{l}8 \text { (by } \\
\text { Aa } \\
\text { LPS) }\end{array}$ & $\underline{N}$ \\
\hline \multirow[t]{2}{*}{$\begin{array}{c}\text { Porphyromonas } \\
\text { gingivalis }\end{array}$} & \multirow{2}{*}{$\begin{array}{l}\text { Extract from } \\
\text { Pg was } \\
\text { chemotactic, } \\
\text { but inhibited } \\
\text { chemotaxis } \\
\text { to other } \\
\text { stimuli }\end{array}$} & \multirow[t]{2}{*}{$\underset{\mathbb{E}}{\bar{E}}$} & $\begin{array}{c}\text { Resists } \\
\text { phagocytosis }\end{array}$ & 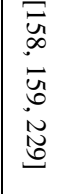 & \multirow[t]{2}{*}{$\begin{array}{c}\text { Minimally } \\
\text { induced }\end{array}$} & \multirow[t]{2}{*}{$\bar{\Xi}$} & \multirow[t]{2}{*}{ MMP9 } & \multirow[t]{2}{*}{$\bar{\Xi}$} & \multirow[t]{2}{*}{$\begin{array}{l}\text { No } \\
\text { effect }\end{array}$} & \multirow[t]{2}{*}{$\bar{\Xi}$} & Not killed & 产 & \multirow[t]{2}{*}{ No effect } & \multirow{2}{*}{ 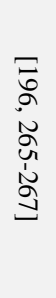 } & \multirow{2}{*}{$\begin{array}{l}\text { IL-1 } \beta \text {, } \\
\text { TNF } \alpha \text {, } \\
\text { IL-8 } \\
\text { and } \\
\text { CCL2 }\end{array}$} & \multirow[t]{2}{*}{ 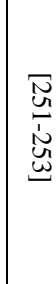 } \\
\hline & & & $\begin{array}{l}\text { Ingested } \\
\text { normally }\end{array}$ & 3 & & & & & & & Killed & 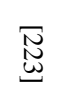 & & & & \\
\hline Filifactor alocis & Inhibits & $\underset{\widetilde{N}}{\mathbb{N}}$ & $\begin{array}{l}\text { Ingested } \\
\text { normally }\end{array}$ & $\sqrt{0}$ & Minimal & $\sqrt{0}$ & $\begin{array}{c}\text { Secretory } \\
\text { vesicles, } \\
\text { Gelatinase } \\
\& \\
\text { Specific } \\
\text { granules }\end{array}$ & ్ㅗㅇ & $\begin{array}{c}\text { Does } \\
\text { not } \\
\text { induce } \\
\& \\
\text { inhibits } \\
\text { PMA- } \\
\text { induced }\end{array}$ & $\sqrt{n}$ & Not killed & N & $?$ & & $\begin{array}{l}\text { IL-1ra, } \\
\text { CCL4 }\end{array}$ & 㥑 \\
\hline $\begin{array}{c}\text { Peptoanaero- } \\
\text { bacter stomatis }\end{array}$ & $\begin{array}{c}\text { Induces } \\
\text { migration } \\
\text { of } \\
\text { neutrophils }\end{array}$ & $\underset{\underline{\omega}}{\widetilde{\sim}}$ & $\begin{array}{c}\text { Resists } \\
\text { phagocytosis }\end{array}$ & $\sqrt[N]{\mathcal{N}}$ & Robust & $\stackrel{\sqrt[N]{U}}{3}$ & $\begin{array}{l}\text { Robust, } \\
\text { all } \\
\text { granules }\end{array}$ & $\stackrel{\sqrt[N]{ }}{\mathrm{J}}$ & Induced & $\sqrt{N}$ & Killed & $\stackrel{\sqrt[N]{3}}{3}$ & $?$ & & $\begin{array}{c}\text { IL-1 } \beta, \\
\text { TNF } \alpha \text {, } \\
\text { IL-1ra, } \\
\text { CXCL1, } \\
\text { CCL2, } \\
\text { CCL3, } \\
\text { CCL4 }\end{array}$ & 恚 \\
\hline
\end{tabular}




\section{CHAPTER 2:}

\section{WHOLE TRANSCRIPTOME ANALYSIS REVEALS THAT FILIFACTOR ALOCIS MODULATES TNF $\alpha$-STIMULATED MAPK ACTIVATION IN HUMAN NEUTROPHILS.}

\section{Introduction:}

Periodontitis is a chronic inflammatory disease where inflammophilic pathogenic bacterial communities accumulate at the gingival crevice. These dysbiotic microbial communities induce a severe inflammatory response that fails to control bacterial growth and contributes to the irreversible destruction of the tooth-supporting tissues [8]. Historically, periodontal research has focused on the pathogenic members of the 'red complex, which includes Porphyromonas gingivalis, Treponema denticola, and Tannerella forsythia. However, recent human microbiome studies have revealed many previously uncultured organisms with a strong correlation with periodontal disease $[36,38]$. One of these newly appreciated species is Filifactor alocis, a Grampositive anaerobic rod whose pathogenic potential and contribution to periodontal diseases is emerging. F. alocis is consistently and abundantly found in periodontal active lesions [49, 50, 57, 278-280]. Furthermore, F. alocis shares virulence characteristics with other periodontal pathogens such as resistance to oxidative stress, biofilm formation, secretion of proteases, and evasion of the immune system $[48,58,59,62,73]$. 
Neutrophils constitute an overwhelming majority of the leukocytes recruited to the oral cavity, where they are essential for maintaining homeostasis of periodontal tissues [119, 281, 282]. Neutrophils can deploy several strategies to efficiently detect, detain and destroy microbes. These include phagocytosis, release of antimicrobial enzymes or toxic factors, generation of massive amounts of reactive oxygen species (ROS) and the discharge of their nuclear material into neutrophil extracellular traps (NETs) [283]. However, oral pathogens have evolved mechanisms to manipulate neutrophil functional responses to prevent being killed while propagating inflammation [14, 282]. Previous work from our laboratory has shown that despite efficient phagocytosis by neutrophils, $F$. alocis survives within neutrophils by inducing minimal production of intracellular ROS and curtailing the fusion of antimicrobial granules with its phagosome [275, 284]. However, in comparison to the keystone oral pathogen, $P$. gingivalis, and another emerging oral pathogen, Peptoanaerobacter stomatis, challenge with $F$. alocis resulted in a mild release of neutrophil-derived pro-inflammatory cytokines, which resulted in limited recruitment of monocytes and other neutrophils [253]. Thus, I hypothesize that $F$. alocis may modulate neutrophil signaling events to interrupt pro-inflammatory cytokine production and alter immune cell recruitment and communication.

The Mitogen-activated protein kinases (MAPKs) are evolutionarily conserved regulators that carry out signal transduction for many cellular functional processes. MAPK activation cascades are well characterized, and usually begin with the ligation of cell surface receptors followed by activation of a relay cascade of phosphorylation of three core kinases: MAP3K, MAP2K (MEK or MKK) and MAPK. Active MAPKs can phosphorylate a variety of intracellular targets, including transcription factors, nuclear pore proteins, 
membrane transporters, cytoskeletal elements, and other protein kinases, so their activation is subjected to spatiotemporal regulation by complex feedback and crosstalk mechanisms [285, 286]. In human neutrophils, bacterial lipopolysaccharide (LPS) activates Toll-like Receptor (TLR) 4 followed by downstream activation of MAPK signaling pathways and the transcription factor regulator nuclear factor (NF)- $\mathrm{kB}$; both of which can independently regulate the production of inflammatory cytokines and chemokines [287, 288]. Both p38 MAPK and ERK pathways control transcription and translation of inducible cytokines in neutrophils stimulated with LPS or TNFa [289]. Due to the relevant role that MAPK signaling plays in regulation of immune responses, it is not surprising that some pathogens have developed mechanisms to hijack this signaling cascade on immune cells [290, 291]. For example, Mycobacterium tuberculosis acetylates a MAPK phosphatase, DUSP16, to increase phosphatase activity on Janus kinase (JNK) and limit inflammatory cytokine production by bone marrow-derived macrophages [292]. Prior work from our group showed that $F$. alocis initially activates both p38 MAPK and ERK1/2 through TLR2 [275]; however, it is unknown what the MAPK response is after $F$. alocis stimulation for longer time points or how the cells respond to secondary stimuli after $F$. alocis challenge.

Few sequencing studies have tracked transcriptome changes in human neutrophils during challenge with a bacterial pathogen [293-296]. Even fewer studies have measured changes in the neutrophil transcriptome associated with challenge by putative oral pathogens. Thus, I sought to characterize global changes at the gene expression level in human neutrophils during infection with $F$. alocis. Analysis of whole-transcriptome by RNA-based next-generation sequencing (RNAseq) shows that $F$. alocis challenge alters the human neutrophil transcriptome by inducing significant changes in the expression of 
genes involved in various neutrophil effector functions. One of the findings of our RNAseq screen was that $F$. alocis challenge affected the expression of components in both the TNF and MAPK kinase signaling pathways. This resulted in decreased p38 MAPK activation by secondary stimuli TNF $\alpha$, but not fMLF. Moreover, only live $F$. alocis limited the TNF $\alpha$-stimulated production of IL-8, demonstrating that this is one of the mechanisms actively induced by the oral pathogen to control neutrophil functional responses.

\section{$\underline{\text { Materials \& Methods: }}$}

Human neutrophil isolation: Human donor recruitment, blood draws, and the use of the materials required for this procedure were in accordance with the guidelines approved by the Institutional Review Board of the University of Louisville. Neutrophils were isolated from venous blood of healthy donors using plasma-Percoll gradients, as described previously [297]. Neutrophil populations were further enriched to obtain highly pure cells (>99\%) by negative magnetic selection using the Easy Eights EasySep Magnet and human neutrophil enrichment kit (Stemcell Technologies, Vancouver, BC, Canada), as previously described [298]. Cell purity was assessed by simultaneously staining with FITCconjugated anti-CD66b (clone G10F5; BioLegend, San Diego, CA, USA) and APCconjugated anti-CD16 (clone CB16; eBioscience, San Diego, CA, USA) antibodies and determining the percentage of $\mathrm{CD}_{6} 6 \mathrm{~b}^{+} \mathrm{CD} 16^{+}$cells using BD Celesta flow cytometer (BD Biosciences, San Jose, CA, USA). Both pure (>90-95\%) and highly pure (>99\%) neutrophils were cultured in complete RPMI-1640 medium (Sigma-Aldrich, St. Louis, MO, USA) with 5\% human serum (Atlanta Biologicals, Flowery Branch, GA, USA). 
Bacterial strains and growth conditions: F. alocis ATCC 38596 was cultured in brain heart infusion (BHI) broth supplemented $5 \mathrm{mg} / \mathrm{mL}$ yeast extract, L-cysteine $(0.05 \%)$ and arginine $(0.05 \%)$ for 7 days anaerobically at $37{ }^{\circ} \mathrm{C}$ as previously described $[275,276]$. Serum opsonization was performed by incubating $F$. alocis at $37^{\circ} \mathrm{C}$ for $20 \mathrm{~min}$ in $10 \%$ normal human serum (Complement Technology, Inc., Tyler, TX, USA). Heat killed $F$. alocis was generated by incubation at $90{ }^{\circ} \mathrm{C}$ for $60 \mathrm{~min}$. Non-viability was confirmed by incubation in culture media at same conditions used for the live organism.

F. alocis challenge and RNA isolation: Highly pure (>99\%) human neutrophils (10-20 $\times$ $10^{6}$ cells $/ \mathrm{mL}$ ) were unstimulated or challenged with opsonized $F$. alocis at a multiplicity of infection (MOI) of 10 , for 1,3 , or $6 \mathrm{~h}$. The infection was synchronized by centrifugation at $14^{\circ} \mathrm{C}$ for $4 \mathrm{~min}$ at $600 \times g$. After each time point, the cells were harvested using Trizol (Life Technologies, Carlsbad, CA, USA) and stored at $-80^{\circ} \mathrm{C}$ until RNA extraction. RNA was isolated from unstimulated and $F$. alocis challenged human purified neutrophils using the hybrid method of Trizol and RNeasy minikit (Qiagen, Venlo, Netherlands). The aqueous phase containing RNA was loaded on the Qiagen column for further purification of RNA. The purified RNA quality was measured by running sample on Bioanalyzer.

Library Preparation: The isolated RNA was checked for integrity using the Agilent Bioanalyzer 2100 system (Agilent Technologies, Santa Clara, CA, USA) and quantified using a Qubit fluorometric assay (Thermo Fisher Scientific, Waltham, MA, USA). Total RNASeq libraries were prepared following Illumina's TruSeq Stranded Total RNA LT 
with Ribo-Zero Gold library preparation protocol (Illumina Inc., San Diego, CA, USA, Cat\# RS-122-2301). After depletion of ribosomal RNA, all samples were ligated with Illumina adapters and individually barcoded. Absence of adapter dimers and consistent library size of approx. $300 \mathrm{bp}$ was confirmed using the Agilent Bioanalyzer 2100. Library quantitation was performed by qPCR using the KAPA Library Quantitation Kit (Kapa Biosystems, part of Roche Sequencing and Life Science, Wilmington, MA, USA) for Illumina Platforms.

Sequencing run: $1.8 \mathrm{pM}$ of the library pool was loaded with $1 \%$ PhiX spike-in on two NextSeq 500/550 75 cycle High Output Kit v2 sequencing flow cells. Sequencing was performed on the Illumina NextSeq 500 sequencer targeting 50M 1x75bp reads per sample.

Bioinformatic analysis: Each of four single-end raw .fastq files for each replicate was concatenated into one single end .fastq file using the unix cat command. A total of sixteen files representing four independent donors and four experimental conditions were generated. Quality control (QC) of the raw sequence data was performed using FastQC (version 0.10.1) [47]. The interquartile range remained above $30(99.9 \%$ base call accuracy) across the reads. The concatenated sequences were directly aligned to the Homo sapiens reference genome assembly (hg38.fa) using tophat2 (version 2.0.13) [300], generating alignment files in bam format. The alignment rate ranged from 88 to 93 percent across the samples. Differential expression analysis between each treatment condition $(1 \mathrm{hr}$, $3 \mathrm{hrs}$, and 6hrs) and the control condition was performed using cufflinks cuffdiff2 (version 2.2.1) [301, 302]. A q-value cutoff $\leq 0.05$ with an absolute $\left|\log _{2} \mathrm{FC}\right| \geq 1$ was used to determine differential expression. 
Reverse Transcription and quantitative real-time PCR (RT-qPCR): Total RNA isolated from the different experimental conditions was followed by reverse-transcription into cDNA using a High capacity RNA to cDNA kit (Applied Biosystems, Foster City, CA, USA), while qPCR was carried out using SYBR®Green PCR Master Mix Applied Biosystems, Foster City, CA, USA) on an Applied Biosystems StepOne Plus cycler with stepone software V2.2.2. Sequences of the gene-specific primers (Integrated DNA Technologies, Skokie, IL, USA) used in this study are listed in Table 2.1. Data were calculated and expressed as mean normalized expression (MNE) units after GAPDH normalization as previously described [303].

Western Blotting: Neutrophils $\left(10 \times 10^{6}\right.$ cells $\left./ \mathrm{mL}\right)$ were cultured at $37^{\circ} \mathrm{C}, 5 \% \mathrm{CO}_{2}$ in RPMI1640 with 5\% heat-inactivated human serum and left unstimulated, stimulated with FSL (100 ng/mL), challenged with either live or heat-killed $F$. alocis for 1, 3, 6, or 10 hours followed by stimulation with fMLF (300 nM, $1 \mathrm{~min})$ or TNF- $\alpha$ (10 ng/ml, $15 \mathrm{~min})$. After the different experimental procedures, cells were centrifuged at $6,000 \times g$ for $30 \mathrm{~s}$ and lysed for $30 \mathrm{~min}$ on ice in ice-cold lysis buffer (20 mM Tris- $\mathrm{HCl}$ [pH 7.5], $150 \mathrm{mM} \mathrm{NaCl}, 1 \%$ [vol/vol] Triton X-100, 0.5\% [vol/vol] Nonidet P-40, 20 mM NaF, 20 mM NaVO3, $1 \mathrm{mM}$ EDTA, $1 \mathrm{mM}$ EGTA, $5 \mathrm{mM}$ phenylmethylsulfonyl fluoride [PMSF], $21 \mu \mathrm{g} / \mathrm{ml}$ aprotinin, $5 \mu \mathrm{g} / \mathrm{ml}$ leupeptin, and 4mM Diisopropyl fluorophosphates [DFP]). After protein estimation using the Pierce BCA protein assay kit (Thermo Scientific, Waltham, MA, USA), samples were adjusted to a concentration of $2 \mu \mathrm{g} / \mu \mathrm{L} .16-20 \mu \mathrm{g} / \mu \mathrm{L}$ of total cell lysates were separated by $12 \%$ SDS-PAGE and immunoblotted with antibodies for 
phospho-ERK1/2, total ERK1/2, phospho-p38 MAPK, total p38 MAPK, phospho-AKT, total AKT, phospho-S6 (Cell Signaling Danvers, MA, USA), p47phox or p67phox (gift from Dr. William M. Nauseef) all at 1:1000 dilution. The appropriate secondary antibodies were used at 1:2000 dilution (Cell Signaling, Danvers, MA, USA). The ECL system (Amersham Pharmacia Biotech, Little Chalfont, United Kingdom) or the SuperSignal West Femto Maximum Sensitivity Substrate (Thermo Scientific,Waltham, MA, USA) was used to visualize antigen-antibody reactions. Densitometric values of each band were calculated using Image Lab software (BioRad, Hercules, CA, USA).

Superoxide Generation and Priming: Superoxide anion release was measured spectrophotometrically at $550 \mathrm{~nm}$ as the superoxide dismutase-inhibitable reduction of ferricytochrome $\mathrm{c}$ as previously described [297]. Briefly, neutrophils $\left(4 \times 10^{6} \mathrm{cells} / \mathrm{ml}\right)$ were cultured in RPMI supplemented with 5\% heat inactivated human serum, and left untreated or pre-treated with p38 inhibitor BIRB-796 $(75 \mathrm{nM}$, added to media $60 \mathrm{~min}$ before 6 and 10 hour timepoints; Sigma, St. Louis, MO, USA), or with opsonized F. alocis (MOI 10) for 6 and 10 hours at $37^{\circ} \mathrm{C}$ in a shaking waterbath. After this first pre-treatment, $\mathrm{TNF} \alpha(10 \mathrm{ng} / \mathrm{ml}, 10 \mathrm{~min})$ were added to all the samples. Samples were run in duplicate, one of the duplicates was used to detect basal superoxide levels in the presence or absence of each pre-treatment, the other sample was used to measure TNF-priming by further challenge with fMLF ( $300 \mathrm{nM}$ ) for 5 min. After stimulation of superoxide production, the samples were centrifuged for $10 \mathrm{~min}$ at $600 \times \mathrm{g}, 4{ }^{\circ} \mathrm{C}$, supernatants were collected, and optical densities were read. 
IL-8 Cytokine Measurement and Apoptosis: Neutrophils $\left(10 \times 10^{6}\right.$ cells $\left./ \mathrm{mL}\right)$ were cultured in RPMI $+5 \%$ heat inactivated human serum, and left untreated, or pre-treated with TAK1 inhibitor (5Z)-7-Oxozeanol $(3 \mu \mathrm{M}$, added 30 min before 6 and 10 hour timepoints; Cayman, Ann Arbor, MI, USA), or p38 inhibitor BIRB-796 (75 nM, added 60 min before 6 and 10 hour timepoints; Sigma, St. Louis, MO, USA), or opsonized F. alocis (MOI 10) for 6 and 10 hours at $37{ }^{\circ} \mathrm{C}$ in an incubator with $5 \% \mathrm{CO}_{2}$. After the pre-treatment, the volume in the tube was divided evenly between two tubes, one of the tubes received TNF $\alpha$ $(10 \mathrm{ng} / \mathrm{ml})$ and the other nothing. All tubes were returned to the incubator for 4 or 12 hours. After the TNF $\alpha$ stimulation period, cells were centrifuged, their supernatants collected and the pellets tested for apoptosis. $1 \%$ protease and phosphatase inhibitors were added to the supernatants to protect them from degradation. IL- 8 was measured in the supernatants using a commercially available kit (Invitrogen, Carlsbad, CA, USA). Cells were processed for Annexin V/7-AAD staining using the commercially available APC Annexin V Apoptosis Detection Kit with 7-AAD (BioLegend, San Diego, CA, USA). Samples were read on a BD FACSCelesta flow cytometer and analyzed using the FlowJo software (Ashland, OR, USA).

Statistical Analysis: Unless otherwise noted, statistical differences among experimental conditions and time points were analyzed by a repeating measures two-way ANOVA, followed by Bonferroni post-tests using GraphPad Prism Software (Graphpad San Diego, CA, USA). Differences were considered significant at the level P $<0.05$. When a two-way ANOVA was not applicable, one-way ANOVA followed by the post-hoc Tukey multiplecomparison test was used. 


\section{$\underline{\text { Results: }}$}

\section{F. alocis induces global changes in gene expression}

To assess changes in gene expression after $F$. alocis challenge, whole transcriptome by RNA-based next-generation sequencing (RNA-seq) was performed on human neutrophils from 4 individual healthy donors that were either left unstimulated or challenged with $F$. alocis for 1, 3 or 6 hours. All time points for each donor were mapped onto a principal component analysis (PCA) plot to determine the variation in the dataset (Figure 2.1A). All four donors clustered together for each experimental condition, showing that donor variability is not a major contributor in our dataset. Contrastingly, the transcriptional profile of $F$. alocis-stimulated neutrophils clearly separated from basal conditions at each time point. Next, differential expression analysis was completed between each treatment condition using the Tuxedo Suite Program cuffdiff 2 , where a p-

value cutoff $\leq 0.01$ and a $\log$ fold change $\left|\log _{2} \mathrm{FC}\right| \geq 1$ was utilized to compile a list of differentially expressed genes (DEG) for further analyses. Volcano plots for each time point showed that $F$. alocis challenge induced a time-dependent change in gene expression, as the number of red colored dots, which represent the significant DEG, grew at each time point (Figure 2.1B). On the volcano plots, the most significant DEG for each time point were identified. Out of these highlighted DEGs, CAMK1G was upregulated and TNFRSF12A was downregulated at all three time points compared to unstimulated cells. The CAMK1G gene encodes a protein like calcium/calmodulin dependent protein kinase; however, according to RefSeq, its exact function is unknown. TNFRSF12A, also known as Fn14, is a weak inducer of apoptosis that can activate NF-kB signaling pathways, 
promotes oxidative stress, and is linked to high expression of matrix metalloprotease 9 (MMP-9) [304-307].

The number of DEG was determined for each time point and plotted based on whether it was upregulated or downregulated compared to the basal control (Figure 2.1C). The biggest change in transcriptome occurred early in the time course, with 624 genes differentially expressed at one hour and a steep increase in the number of DEG between 1 and 3 hours (Figure 2.1D). At the 6-hour time point, the number increased only marginally from 3 hours. Throughout the time course, the number of genes induced was roughly the same as the number of repressed genes. While our DEG criteria is more stringent than other studies, these global changes in gene expression appear to be unique to $F$. alocis as compared to other transcriptome studies between neutrophils and bacterial challenge [293296]. To validate the RNAseq data, 2 upregulated genes, 2 downregulated genes and 2 genes with no change were selected for validation by quantitative PCR. Figure 2.1E shows the Fragments Per Kilobase of transcript per Million mapped reads (FPKM) expression values for all four donors from the RNAseq screen, while Figure 2.1F shows the mean normalized mRNA expression by qPCR. Overall, the qPCR results validate the RNAseq screen and provide confidence about the targets identified by the high throughput screening analysis.

\section{F. alocis affects neutrophil functional and biochemical processes}

To reduce bias during the bioinformatic analysis, the list of DEG was uploaded into

two separate databases: Database for Annotation, Visualization and Integrated Discovery (DAVID) [308, 309] and MetaCore by Clarivate Analytics. In each 
database, I first identified the significant $(\mathrm{p}<0.01)$ biological processes during challenge with $F$. alocis. From the DAVID analysis, 37, 74, and 86 processes were identified for the 1-, 3- and 6-hour time points, respectively, and categorized by cell function (Table 2.2). Significant process categories in every time point include biological processes related to the inflammatory response, response to microbes, chemotaxis, signal transduction, gene expression and transcription factor regulation, cytokine-mediated responses and production, and apoptosis. However, as the time course progressed, there was a shift in the affected processes. While cytokine-related processes were most prominent at the earlier timepoints, biological processes related to phagosome maturation and metabolic processes became significant at the later time points. Moreover, processes related to protein folding only became significant at 6 hours post bacterial challenge.

Using MetaCore, 71 significant $(\mathrm{p}<0.01)$ network processes were determined in our data set. Since the MetaCore software automatically categorizes the processes by cell function, I determined the frequency of each category (Figure 2.2A). Like the DAVID analysis, most processes were involved with inflammation, signal transduction, the immune response, and apoptosis. Cell function processes with a lower frequency include protein folding, cytoskeleton, transcription, chemotaxis, and autophagy. Next, I plotted the top 25 most significantly upregulated (Figure 2.2B) and downregulated processes (Figure 2.2C). Processes related to inflammation made up 4 out of the top 5 upregulated network processes, but the significance of the inflammatory processes decreased as the time course progressed. In fact, some of these inflammation processes from the upregulated list became significant in the downregulated processes during the later time points, as is the case with processes such as IL-6 signaling and neutrophil activation (Table 2.3). This suggests that 
F. alocis may be dampening inflammatory processes between 1 and 3 hours to prolong its survival or provide protection to bystander species. Together, this data shows that $F$. alocis challenge induces temporal changes in neutrophil functional mechanisms like cytokine production, chemotaxis, vesicular trafficking and degranulation, as well as neutrophil biochemical mechanisms like the regulation of signaling pathways and metabolism. This coincides with previous data from our laboratory that shows that $F$. alocis affects neutrophil cytokine production, chemotaxis, vesicle trafficking, and degranulation functions $[253,284]$.

Based on previous work that demonstrated that $F$. alocis induces minimal intracellular and extracellular ROS production [284], I looked at whether the components of the NAPDH oxidase complex are affected during $F$. alocis challenge (Table 2.4). From the RNAseq data, the only statistically significant results show that the expression of CYBB (gp91phox subunit) increased in a time-dependent manner while the expression of NCF1 (p47phox subunit) decreased by 6 hours of challenge, which was also validated by qPCR (Figure 2.1F). While the minimal ROS activation at the early time points cannot be attributed to modulation of gene expression by F. alocis, generation of ROS at later time points may be inhibited by the expression of a member of the galectin family of carbohydrate binding proteins, galectin 3 . The increased expression of galectin 3 , has already been associated with inhibition of ROS production when human neutrophils were challenged with Candida albicans [310]. In our dataset, both the FPKM expression as well as the qPCR validation show a time dependent increase in galectin-3 mRNA expression (Figure 2.1E-F), and when tested by western blot, F. alocis induced a time dependent increase in galectin-3 protein expression (data not shown). 
F. alocis challenge upregulates cytokine pathways and downregulates signaling pathways

Next, I identified pathways relevant to challenge with $F$. alocis. Using DAVID, the list of DEG was mapped onto predefined pathways from the Kyoto Encyclopedia of Genes and Genomes (KEGG) database. I limited our analysis to highly significant pathways with a $\mathrm{p}$ value $<0.01$, which resulted in 10,26 , and 33 pathways for the 1,3 , and 6-hour time points, respectively (Table 2.5). The F. alocis-neutrophil transcriptome reinforced the pathogenic potential of $F$. alocis by the number of significant pathways linked to pathogens that subvert immune cells (Salmonella, Legionella, Helicobacter pylori, and Influenza A). Similarly, pathways for cancers, rheumatoid arthritis, and inflammatory bowel disease were significant for the $F$. alocis-challenged neutrophil transcriptome in both databases. Oral bacteria continue to be linked to systemic malignancies like those listed above [311], and although $F$. alocis has not been amongst the oral pathogens detected yet, these results hint that it could play a role in the pathogenesis of these diseases.

Two major bacterial recognition receptor signaling pathways were identified in our data set, NOD-like receptor and Toll-like receptor signaling. These receptor pathways align with published data on $F$. alocis-induced cytokine production, where NOD1 is activated during challenge with heat-killed $F$. alocis to produce IL-6 in monocytes [68], and TLR2/6 activation of neutrophils leads to the production and release of cytokines and chemokines [253]. Using Metacore, I divided the significant pathways into the top 20 upregulated (Figure 2.3A) and downregulated (Figure 2.3B) plots. The list of upregulated pathways supports published data that shows initial contact with $F$. alocis results in the early transcription and production of cytokines [253]. At 1 hour, cytokine-related pathways such 
as cytokine-cytokine receptor interaction, TNF signaling pathway, and chemokine signaling pathway were also prominent pathways by KEGG analysis (Table 2.5). In both databases, the NFкB signaling pathway was significant and upregulated, suggesting this transcription factor is responsible for the cytokine and chemokine transcriptome response. While many of the upregulated pathways were related to inflammation and cytokine responses, the downregulated list was largely comprised of signal transduction pathways (Figure 2.3B). The pathways were significantly affected at the later timepoints and include signal transduction by MAPK, GPCR, Rho GTPases, PI3K, PTEN, AKT, and PKA. Out of the list of 20 downregulated pathways, seven relate to MAPK signaling. To further support this analysis, under the signal transduction biological process category, positive regulation of ERK1 and ERK2 cascade and activation of MAPK activity are significant at one hour, but at 3 and 6 hours, inactivation of MAPK activity becomes significant (Table 2.5). I focused on this pathway and determined if $F$. alocis is modulating MAPK signaling in human neutrophils.

\section{$\underline{\text { F. alocis challenge does not affect fMLF-stimulated MAPK signaling }}$}

Since G-protein coupled receptor (GPCR) and MAPK were both hits in our dataset, western blots to evaluate ERK1/2 and p38 MAPK activation were performed on lysates from human neutrophils pretreated with media or media containing $F$. alocis for 1, 3, 6, and 10 hours followed by stimulation with the bacterial peptide N-Formylmethionineleucyl-phenylalanine (fMLF) (Figure 2.4A). Densitometry analysis of the western blots bands for phosphorylated and total ERK1/2 showed that stimulation with $F$. alocis alone has a time dependent increase in phosphorylation of ERK1/2 (Figure 2.4B). This suggests 
there is a bimodal response in the activation of ERK1/2 since it was previously published that ERK phosphorylation peaks at $15 \mathrm{~min}$ and then decreases [275]. In the case of p38 MAPK, the levels of phosphorylated p38 MAPK are also increased in the $F$. alocis pretreated cells as compared to neutrophils cultured in media alone (Figure 2.4C). However, the levels remain steadily elevated throughout the time course. This pattern of phosphorylation for the MAPK is also observed, although at different magnitudes, when neutrophils are pre-treated with heat-killed $F$. alocis (Figure 2.4D, E), or the TLR2/6 agonist FSL1 (Figure 2.4F, G). Despite the increased basal levels of phosphorylated ERK1/2 and p38 MAPK in the viable and heat-killed $F$. alocis pretreated cells, when the neutrophils are stimulated with fMLF after pre-treatment with $F$. alocis, the phosphorylation of both ERK and p38 MAPK is comparable to that of cells cultured in media + fMLF (Figure 2.4B, C). Contrastingly, cells pretreated with FSL1 showed increased phosphorylation of ERK1/2 when stimulated with fMLF, which became significant at 10 hours compared to media-cultured cells stimulated with fMLF alone (Figure 2.4F). A similar trend was observed at the 10-hour time point with phosphorylation of p38 MAPK but the data did not reach statistical significance when compared to fMLF alone (Figure 2.4G).

\section{F. alocis challenge dampens TNF- $\alpha$-stimulated MAPK signaling}

From the DAVID analysis, one of the KEGG pathways that was significantly modulated by $F$. alocis at each timepoint was the TNF signaling pathway (Table 2.5). Similarly, four of the upregulated pathways and one of the dowregulated pathways from the MetaCore analysis involve TNF signaling (Figure 2.3A, B). Since it is well documented 
that stimuli like LPS and TNF $\alpha$ can activate the $\mathrm{p} 38$ and MEK/ERK pathways in neutrophils [287, 312,313] and high levels of TNF $\alpha$ are present in periodontitis active sites [314, 315], I tested the effect of $F$. alocis pre-treatment on TNF $\alpha$-induced MAPK signaling cascade. Whole cell lysates from neutrophils pretreated with media or $F$. alocis followed by stimulation with TNF $\alpha$ were immunoblotted for phosphorylated and total ERK1/2 and p38 MAPK (Figure 2.5A). Densitometry analysis of the ERK immunoblots showed that pre-treatment with $F$. alocis did not impact TNFa-driven phosphorylation of ERK1/2 (Figure 2.5B). However, the TNF $\alpha$-driven phosphorylation of p38 MAPK was significantly dampened in neutrophils pre-treated with F. alocis for 6 and 10 hours as compared to neutrophils cultured in media alone (Figure 2.5C). This effect is dependent on the bacteria being viable, because when the neutrophils were pre-treated with heat-killed F. alocis before stimulation with $\mathrm{TNF} \alpha$, there was no decrease in the $\mathrm{p} 38$ phosphorylation at 6 or 10 hours (Figure 2.5D). Additionally, ligation of TLR2/6 is insufficient to elicit the phenotype observed (Figure 2.5E). This data shows that viable $F$. alocis modulates TNF $\alpha$ induced activation of the MAPK signaling pathway by selectively interfering with the phosphorylation of p38 MAPK, but not ERK1/2.

In human neutrophils, activation of p38 MAPK by TNF $\alpha$ results in the downstream phosphorylation and activation of AKT [316]. Therefore, since the TNF $\alpha$-induced activation of p38 MAPK was affected when neutrophils were pre-treated with $F$. alocis, the activation of AKT should also be compromised. To test this hypothesis, the lysates from media or F. alocis pre-treated neutrophils stimulated with TNFa were immunoblotted for phosphorylated and total AKT (Figure 2.6A). Densitometry analysis of the Western blots demonstrated that TNF $\alpha$-mediated phosphorylation of AKT was also dampened in $F$. 
alocis treated cells as compared to media-cultured neutrophils (Figure 2.6B). The reduced AKT phosphorylation followed the timing of the decreased p38 MAPK phosphorylation with the phenotype reaching statistical significance only at 6 and 10 hours. Like the p38 MAPK phenotype, the lowered AKT activation was dependent on interaction with viable F. alocis (Figure 2.6C) and was not mediated solely through ligation of the TLR2/6 receptor (Figure 2.6D). Also downstream of p38 MAPK phosphorylation is the activation of translation machinery such as the S6 ribosomal protein [287]. Thus, the phosphorylation of S6 was tested in whole cell lysates from neutrophils pre-treated with $F$. alocis prior to TNF $\alpha$ stimulation (Figure 2.7A). Densitometry analysis showed that the activation of S6 in response to TNF $\alpha$ was significantly dampened in neutrophils pre-treated with $F$. alocis for 6 hours in comparison to media treated controls (Figure 2.7B). While this trend continued in the 10-hour pre-treatment condition, it did not reach statistical significance. Together, these results demonstrate that $F$. alocis actively modulates the TNF $\alpha$ signaling pathway by dampening the activation of p38 MAPK and its downstream effectors, AKT and S6 ribosomal protein.

\section{Functional effects of $\mathrm{F}$. alocis' inhibition of TNFa-mediated p38 phosphorylation}

TNF $\alpha$ stimulation can prime the ROS response of neutrophils, extend their lifespan and induce cytokine production (reviewed in [127]). To determine if the interference of TNF $\alpha$ signaling resulted in any phenotypic changes, these three TNF $\alpha$-mediated functional responses were tested on cells cultured with $F$. alocis for 6 and 10 hours prior to TNF $\alpha$ stimulation. The RNA seq screen showed that the mRNA levels of some NADPH oxidase components were affected; thus, before testing the ROS priming response, the protein 
expression of two of the subunits p47phox (NCF1) and p67phox (NCF2) was determined (Figure 2.8). The RNAseq screen showed the gene expression of $\mathrm{p} 67 \mathrm{phox}$ was unchanged when the cells were challenged with $F$. alocis, but the gene expression of p47phox was significantly decreased in F. alocis treated cells (Table 2.4). At the protein level, there was no significant difference between media cultured neutrophils and those exposed to $F$. alocis at any timepoint tested for either p47phox or p67 (Figure 2.8A-C). Stimulation with TNF $\alpha$ also had no effect on either subunit's protein expression in media cultured neutrophils or those exposed to $F$. alocis, demonstrating that any changes observed in the ROS priming response could not be due to differences in the availability of NADPH oxidase components. The basal extracellular superoxide production was similar in cells cultured in media or in media with p38 inhibitor BIRB-796 or $F$. alocis for 1,6 , and 10 hours (Figure 2.9A). However, when BIRB-796 and $F$. alocis pre-treated cells were primed with TNF $\alpha$ followed by stimulation with fMLF, the superoxide production was comparable to that of neutrophils cultured in media prior to the TNF $\alpha$ priming. Thus, I conclude that inhibiting p38 activation through a chemical inhibitor or $F$. alocis does not affect the ability of $\mathrm{TNF} \alpha$ to prime neutrophils' ROS response.

In human neutrophils, TNF $\alpha$ stimulation activates MAPK kinase kinase, TAK1 (also known as MAP3K7), which leads to the downstream phosphorylation of ERK1/2 to delay apoptosis and the phosphorylation of p38 to induce cytokine production (Figure 2.12) [287, 316]. First, the effect of $F$. alocis- impaired p38 activation was tested on apoptosis. Neutrophils were cultured in media or media with a TAK1 inhibitor (5Z)-7-Oxozeanol (30 min), a p38 inhibitor BIRB-796, (60 min) or F. alocis for 6 hours, followed by $\pm \mathrm{TNF} \alpha$ stimulation for 12 hours (Figure 2.9B, Fig. 2.10). Based on Annexin V and 7-AAD 
staining, TNF $\alpha$ stimulation of cells cultured in media was able to decrease the number of apoptotic cells compared to cells left in media alone. When TAK1 was inhibited by (5Z)7-Oxozeanol, neutrophils became apoptotic, especially when treated with TNF $\alpha$. Neutrophils pre-treated with BIRB-796 behaved similarly to media-cultured neutrophils, where TNF $\alpha$ stimulation is pro-survival because inhibition of $\mathrm{p} 38$ does not affect the TNF $\alpha$-ERK1/2 mediated delay in apoptosis. Interestingly, pre-treatment of neutrophils with $F$. alocis alone resulted in a decrease in apoptotic cells, which was not reduced further with TNFa stimulation. Apoptosis was also assessed in cells pre-treated with $F$. alocis for 10 hours prior to the 12-hour stimulation with $\mathrm{TNF} \alpha$, and results matched the 6-hour pretreatment (Figure 2.11A-E). Together, this data reinforces the finding that only ERK1/2 signalling is important in TNF $\alpha$-induced neutrophil survival and that $F$. alocis is selectively inhibiting p38 MAPK.

$\mathrm{TNF} \alpha$ stimulation can also induce the production of cytokines and chemokines, such as interleukin (IL)-8. Thus, the release of IL-8 was tested in the supernatants of cells cultured with media, (5Z)-7-Oxozeanol, BIRB-796, or F. alocis for 6 hours followed by +/- TNF $\alpha$ stimulation for 12 hours (Figure 2.9C). As expected, TNF $\alpha$ stimulation of mediacultured neutrophils induced significant release of IL-8. Culturing the neutrophils with the TAK1 and p38 inhibitors alone did not induce IL-8 production; however, TAK1 and p38 inhibition reduced the release of IL-8 by TNF $\alpha$ stimulation. Contrastingly, the $F$. alocis pre-treatment alone caused robust release of IL-8, which significantly surpassed the IL-8 release of TNF $\alpha$-activated, media-cultured cells. Despite the potent IL-8 production by $F$. alocis alone, the further stimulation of $F$. alocis pre-treated cells with TNF $\alpha$ did not cause significant, additional release of IL-8. To rule out the possibility that $F$. alocis treatment 
alone exhausted the neutrophils' ability to produce IL-8, I also tested neutrophil IL-8 production after pre-treatment with heat-killed $F$. alocis, which show normal p38 activation in response to TNF $\alpha$ stimulation (Figure 2.5D). Similar to viable bacteria, the heat-killed bacteria induced significant IL-8 production from neutrophils, and this production was not significantly enhanced with stimulation by TNF $\alpha$ (Figure 2.9C). This phenotype was also observed when neutrophils were pre-treated for 10 hours with viable or heat-killed F. alocis prior to $\mathrm{TNF} \alpha$ challenge for 12 hours (Figure 2.11F), suggesting that despite a defect in TNF $\alpha$-mediated p38 phosphorylation by the viable bacterium, alternative pathways are activated by $F$. alocis that result in maximal IL-8 production from neutrophils in the timepoints tested. This observation raised the possibility that the inhibition of p38 phosphorylation by $F$. alocis subsides during the 12-hour TNFa stimulation period. Therefore, I tested the phosphorylation of $\mathrm{p} 38$ in neutrophils pre-treated with media or $F$. alocis for 6, 10 and 20 hours prior to TNFa stimulation for $15 \mathrm{~min}$, and the inhibition of p38 phosphorylation by $F$. alocis had dissipated by 20 hours, suggesting the MAPK dampening by $F$. alocis is a transient effect (Figure 2.9D). Finally, to determine if IL-8 production is affected during the period of infection where p38 phosphorylation is dampened, I shortened the TNF $\alpha$ stimulation to 4 hours (Figure 2.9E). Despite the shorter stimulation period, TNF $\alpha$ still caused significant release of IL-8 from media cultured cells. Both viable and heat-killed $F$. alocis induced significant IL-8 production on their own, but their responses diverged after $\mathrm{TNF} \alpha$ stimulation. While heat-killed $F$. alocis pre-treated cells produced a greater amount of IL-8 upon addition of TNF $\alpha$, viable $F$. alocis-treated cells were incapable of generating more IL-8. Combined, this data demonstrates that viable F. alocis blocks $\mathrm{TNF} \alpha$-mediated $\mathrm{p} 38$ activation to reduce transiently the production of pro- 
inflammatory cytokines, but this interference has no effect on other TNF $\alpha$ induced effector functions like ROS priming or pro-survival response.

\section{Discussion:}

As executioners of the innate immune response, neutrophils are recruited to the gingival tissue to provide the host with protection against infection. However, in active periodontitis disease sites the interaction between neutrophils and the dysbiotic microbial community results in dysregulated inflammation, which is detrimental to the host. Composition analysis of the dysbiotic microbial community identified high concentrations of emerging periodontal pathogens such as $F$. alocis. I recently demonstrated that $F$. alocis survives within human neutrophils by inducing minimal ROS production and blocking granule recruitment to the bacteria-containing phagosome [284]. Despite $F$. alocis causing significant changes in the mRNA expression of different neutrophil-derived cytokines and chemokines, lower levels of these inflammatory mediators are released when compared to the response elicited by other oral pathogens [253]. Therefore, a systems biology-level approach was used to define global changes in human neutrophil transcriptome modulated by $F$. alocis. This unbiased approach provides insights into how this emerging oral pathogen might undermine the innate immune system and contribute to disease progression. Our results show that among the 71 significant biological process modulated by $F$. alocis, the highest percent were related to inflammation, signal transduction, immune response, and apoptosis. Furthermore, the KEGG pathway analysis revealed that uptake of F. alocis significantly downregulated the expression of genes associated with signal transduction pathways primarily the MAPK cascade and the TNF $\alpha$ signaling pathways. To 
the best of our knowledge, our results are the first to show that TNF- $\alpha$-induced p38 MAPK activation is significantly impaired in $F$. alocis-challenged neutrophils.

Research studies from the last 20 years have corrected the misconception that neutrophils were unable to induce changes in gene expression because they are short-lived, differentially terminated cells with a densely condensed nucleus [317-320]. Microarraybased approaches show that significant changes in neutrophil gene expression take place after 3-6 h following microbial uptake [319]. Likewise, our results show a significant increase in neutrophil DEGs from 624 genes up to 2671 following 1 and $3 \mathrm{~h}$ of $F$. alocis challenge, respectively. By $6 \mathrm{~h}$ post bacterial challenge the number of DEGs continued to rise up to 3489 with a similar number of upregulated (1739) and downregulated (1750) genes. Interestingly, the transcriptome studies performed thus far on neutrophils following bacterial interactions reveal common as well as pathogen-specific transcriptional profiles, providing novel information about the potential pathogenic persona of the microorganism studied [320]. For example, Anaplasma phagocytophilum induces minimal ROS production by neutrophils and a microarray study following 1.5 up to 24 h post infection shows that the inability to mount the response is not due to modulation of the genes encoding for the different components of the NADPH oxidase [294]. F. alocis is phagocytized by human neutrophils but induces minimal ROS production [284]. In contrast to the transcriptional neutrophil profile elicited by A. phagocytophilum, our RNAseq and qPCR results indicate a significant downregulation of the gene that encodes for one of the cytosolic components of the NADPH oxidase, p47phox, and a significant time dependent increase in the gene expression of galectin 3. P47phox together with p67phox and p40phox form the triad cytoplasmic complex, in a 1:1:1 stoichiometric ratio, which is essential for 
NADPH oxidase activation [321]. Although the minimal ROS induced by $F$. alocischallenged neutrophils was monitored between 1 and $90 \mathrm{~min}$ and the significant decrease in p47phox gene expression was observed at $6 \mathrm{~h}$ post infection, these pathogen-induced changes in the transcript levels could leave neutrophils defective in mounting an appropriate respiratory burst response. Furthermore, cytosolic galectin 3 acts as a negative regulator of ROS production in both human and mouse neutrophils by modulating complement receptor 3 signaling pathway during C. albicans infections [310]. Our results show a time dependent increase in galectin 3 gene expression which could be one of the strategies used by $F$. alocis to inhibit ROS production. The mechanisms by which $F$. alocis modulates ROS production, both during early and late time points of infection, is an area under investigation in our laboratory.

To mount an efficacious antimicrobial response inside the neutrophil phagosome, the synergy between an optimal activation of the NADPH oxidase, and the fusion of the different neutrophil granule subtypes with the bacteria-containing phagosome is essential [321]. Microbial pathogens manipulate either one or both of these antimicrobial processes to evade neutrophil killing [89]. The expression of CEACAM3-binding opacity (Opa) proteins on Neisseria gonorrhoeae renders the organism susceptible to neutrophil killing. The ability of N. gonorrhoeae to switch off the expression of Opa proteins, by phasevariation, prevents azurophilic granule fusion to the phagosome, thus promoting bacterial survival [322]. During the phagocytic cup formation, effector proteins secreted by Yersinia pseudotuberculosis prevent fusion of specific granules to the forming phagosome in human neutrophils [323]. Our transcriptome results show that $F$. alocis challenge significantly downregulated neutrophil processes and signaling pathways involved in the regulation of 
vesicle-mediated transport, neutrophil degranulation, and cellular pathways involved in transport. This significant downregulation of vesicular trafficking and phagosome maturation transcripts is consistent with our previous results that $F$. alocis inhibits specific and azurophilic granule recruitment to the bacteria-containing phagosome [284]. The mechanisms induced by $F$. alocis to modulate neutrophil vesicular trafficking to the phagosome is an area of active investigation in our laboratories.

Neutrophils isolated from periodontitis patients and from healthy controls were transcriptionally active following $3 \mathrm{~h}$ challenge with Fusobacterium nucleatum, which is found in high numbers in the subgingival plaque from periodontitis patients [295]. In this microarray study, $F$. nucleatum induced significant upregulation of genes encoding proinflammatory cytokines and chemokines, and it has been shown that this organism induces the release of high levels of these inflammatory mediators from neutrophils [197]. In our study I looked at the transcriptional response at 1,3 and 6 hours while the microarray study with $F$. nucleatum was performed only at 3 hours. However, our results show that at $1 \mathrm{~h}$ post $F$. alocis challenge there is a significant increase in genes involved in proinflammatory cytokines such as IL- $1 \beta$ and IL-6 but those same signaling pathways were significantly downregulated by 3 and 6 hours. $F$. alocis induces an early increase in both gene expression and protein release of pro-inflammatory mediators such as IL-1 $\beta, \mathrm{TNF} \alpha$, CXCL1, CXCL8, CCL1, CCL2, and CCL3; but the levels released by human neutrophils are significantly lower compared to the response elicited by $P$. gingivalis and $P$. stomatis [253]. These results suggested that $F$. alocis might modulate the protein expression and/or release of inflammatory mediators by neutrophils, and our current results confirm this hypothesis since $F$. alocis pre-treatment limited the release of IL-8 from TNF $\alpha$ stimulation. 
An interesting observation from the RNAseq analysis was that $F$. alocis modulates MAPK signaling pathways. Activation of the different MAPK pathways play a pivotal role in several inflammatory and antimicrobial functional responses both in macrophages and in neutrophils [291]. Pathogenic organisms have evolved different strategies to modulate MAPK activation by releasing bacterial compounds into the host innate immune cell that cause kinase inactivation by proteolysis, post-translational modification at active enzymatic sites, as well as by induction of different phosphatases [290, 291]. Either inactivation or sustained activation of the MAPK signaling pathways will lead to a dysregulated immune response. $F$. alocis challenge induces an early activation of p38 and ERK1/2 in human neutrophils that peaks between 15-30 min and decreases following 60 min of bacterial challenge [275]. I expanded our initial observation, and the data from the present study revealed that $F$. alocis induces a second phase of MAPK activation in human neutrophils beyond 60 min of bacterial challenge. Besides modulation of MAPK signaling, RNAseq analysis showed that $F$. alocis also induced changes in gene expression associated with GPCR and TNF signaling pathways. In the inflamed gingival crevice environment, neutrophils infected with $F$. alocis will be also exposed to bacterial peptides, such as fMLF, as well as to inflammatory cytokines such as TNF $\alpha$. Our results show a similar degree of fMLF-stimulated p38 and ERK1/2 phosphorylation in human neutrophils in the presence or absence of $F$. alocis infection. However, when TNF $\alpha$ was used as the second stimulus, F. alocis-challenged neutrophils showed a significant decrease in p38 MAPK activation. This modulation of TNF $\alpha$-induced p38 phosphorylation was not seen when neutrophils were exposed to heat-killed $F$. alocis or the TLR2/6 agonist, FSL-1, prior to the cytokine stimulation. Furthermore, the combination of $F$. alocis and $\mathrm{TNF} \alpha$ had no impact on 
ERK1/2 activation by the pro-inflammatory cytokine. While many pathogenic organisms can modulate MAPK signaling in innate immune cells as a mechanism to increase bacterial virulence, this is the first time this observation has been shown for F. alocis.

In human neutrophils, $\mathrm{TNF} \alpha$ stimulation results in activation of both p38 and ERK1/2, which are involved in the production of inflammatory cytokines and chemokines independently of NF-kB activation [316]. However, blocking TNF $\alpha$-induced activation of p38, but not ERK1/2, impaired both the transcription and translation of inflammatory cytokines by human neutrophils [287]. Our results show that TNF $\alpha$-induced activation of p38 MAPK is impaired in neutrophils infected with $F$. alocis, the extent to which this affects functional mechanisms was tested and is summarized in Figure 2.12. This manipulation of MAPK signaling pathway by $F$. alocis limited the release of TNF-induced chemokine IL- 8 by neutrophils. This phenotype has been described for other periodontal pathogens, which employ multiple mechanisms to manipulate IL-8 production and limit the influx of neutrophils [282]. In human neutrophils, stimulation by TNF $\alpha$ also has a prosurvival response. It has been shown that activation of MEK and ERK1/2, which are uncoupled in human neutrophils, participate in the pro-survival effect of TNF $\alpha$ [289]. A previous study also showed that $\mathrm{TNF} \alpha$ activated both $\mathrm{p} 38$ and ERK1/2 in human neutrophils, but that only activation of ERK1/2 was necessary for TNF $\alpha$-mediated inhibition of caspase-3 activity and the pro-survival effect [324]. In our study, F. alocis challenge did not affect TNF $\alpha$-induced ERK1/2 activation, and when apoptosis was tested, the pro-survival effect of the cytokine was not impaired. In fact, stimulation with $F$. alocis alone had a pro-survival effect on neutrophils, which was also reflected in the RNAseq analysis where $F$. alocis up-regulated anti-apoptotic signaling pathways in neutrophils. 
It has been shown that phagocytosis of pathogenic bacteria such as Staphylococcus aureus and Streptococcus pyogenes induces changes in neutrophil gene expression involved in the acceleration of apoptosis; whereas a different transcriptional profile, linked to delay neutrophil apoptosis, is induced following A. phagocytophilum and Francisella tularensis infection [294, 296, 320]. A microarray study showed that $F$. nucleatum induces upregulation of anti-apoptotic genes in human neutrophils [295]. Similarly, our RNAseq analysis identified several upregulated pro-survival and downregulated pro-apoptotic differentially expressed genes in neutrophils after $F$. alocis challenge. Extending neutrophil life span delays cell turnover and prevents resolution of inflammation contributing to disease progression. The mechanisms utilized by $F$. alocis to delay neutrophil apoptosis is an area under current investigation in our laboratory.

In conclusion, $F$. alocis induces significant changes in the human neutrophil transcriptome. In particular, biological processes involved with inflammation, signal transduction, vesicular trafficking, neutrophils activation, and apoptosis were significantly regulated. Furthermore, these results show that $F$. alocis modulated both the TNF and MAPK kinase signaling pathways. This resulted in decreased p38 MAPK activation by a secondary stimulus i.e. TNF $\alpha$, but not by fMLF. F. alocis, by selectively blocking p38 MAPK, but not ERK1/2, by the secondary stimulus TNF will potentially maintain a delay of neutrophil apoptosis, while dampening the release of inflammatory mediators. 
A.

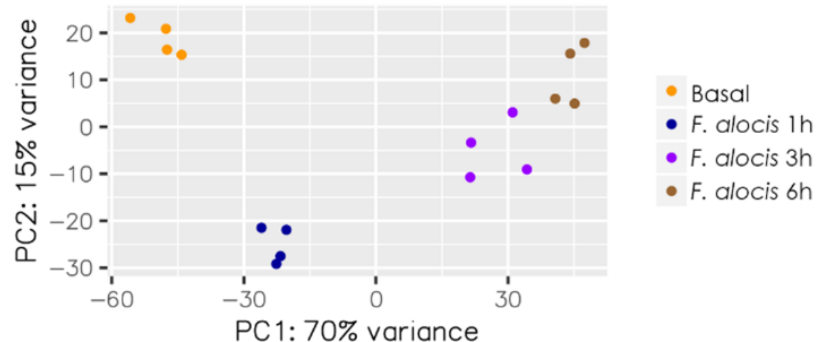

B. $\quad$ Not significant
$\quad$ P value $<=0.01$
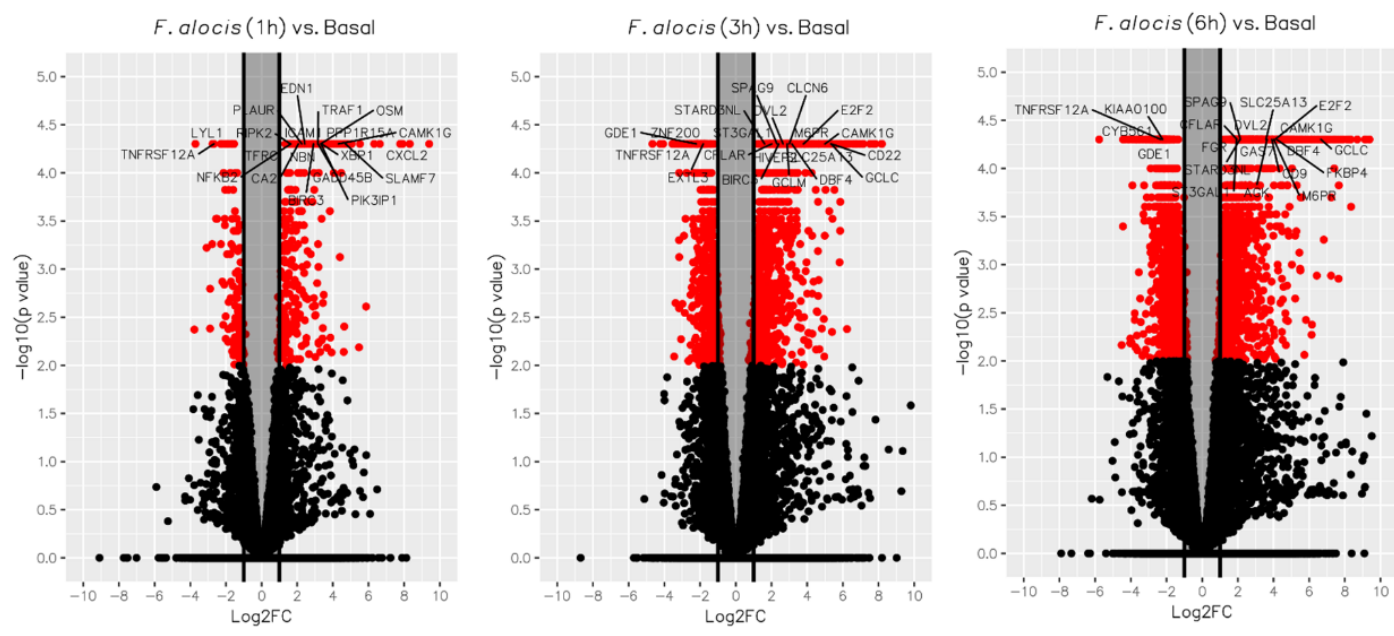

C.

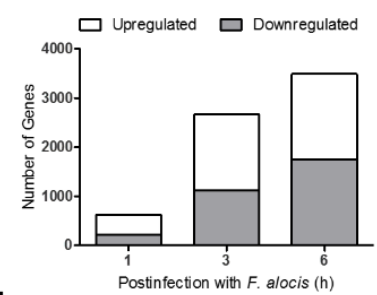

D.

\begin{tabular}{|c|c|c|c|}
\hline $\begin{array}{c}\text { Challenge } \\
(\mathrm{h})\end{array}$ & Total DEG & Upregulated & Downregulated \\
\hline 1 & 624 & 411 & 213 \\
\hline 3 & 2671 & 1546 & 1125 \\
\hline 6 & 3489 & 1739 & 1750 \\
\hline
\end{tabular}

E.

F.
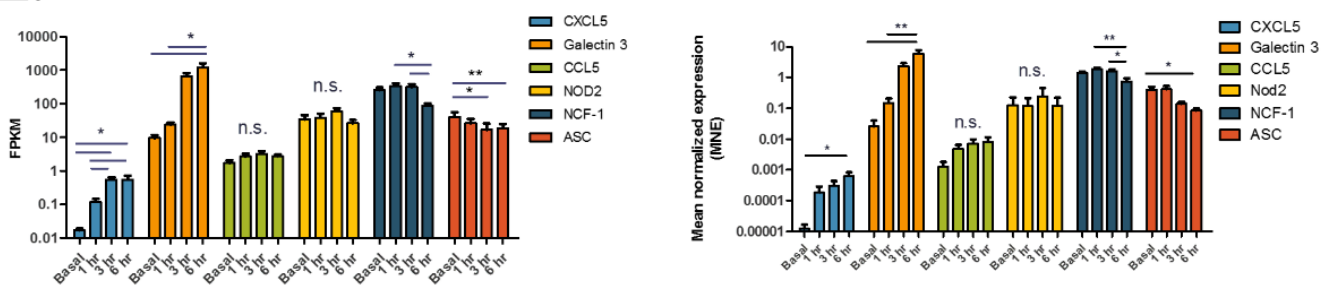

Figure 2.1: Global changes in the transcriptome of $F$. alocis-challenged neutrophils 
Figure 2.1: Illumina RNA sequencing was performed on human neutrophils from 4 individual healthy donors that were either left unstimulated (Basal) or challenged with $F$. alocis for 1, 3 or 6 hours. PCA analysis shows the variation between the four donors at each timepoint (A). The Tuxedo Suite program cuffdiff 2 was used to acquire a list of differentially expressed genes (DEG) that were graphed in volcano plots (B). Genes that had $\mathrm{p} \leq 0.01$ and $\log _{2} \mathrm{FC} \mid \geq 1$ are colored in red and genes with high fold changes are labeled. For each time point, the average number of upregulated and downregulated differentially expressed genes (DEG) was determined and plotted (C, D). Six genes (two upregulated, two with no change, and two downregulated) were chosen to validate the RNAseq data by quantitative qPCR analysis. From the RNAseq data, the fragments per kilobase of transcript per million mapped reads (FPKM) for these six genes are plotted as mean \pm SEM from the four donors $(\mathrm{E})$. From the qPCR analysis, the mean normalized mRNA expression (MNE) from 5 independent experiments are plotted in (F) as MNE \pm SEM. One-way ANOVAs were performed on the expression levels from each gene to determine statistical significance between the basal condition and each timepoint. $*=\mathrm{p}<$ $0.05, * *=\mathrm{p}<0.01, * * *=\mathrm{p}<0.001$ 
A.

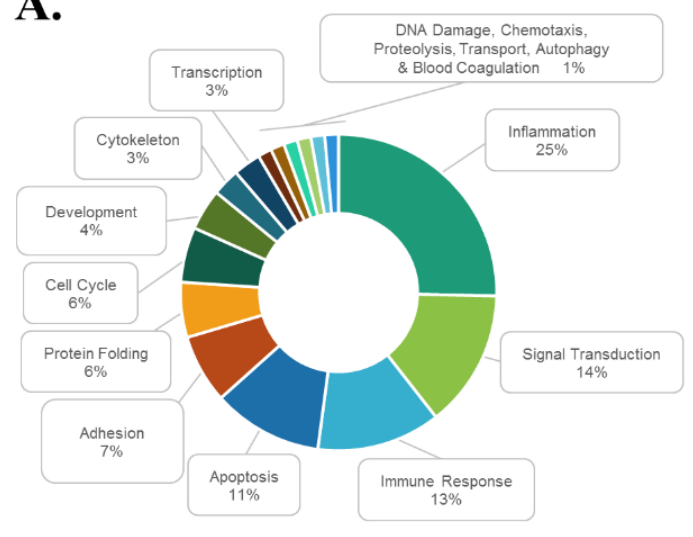

C.

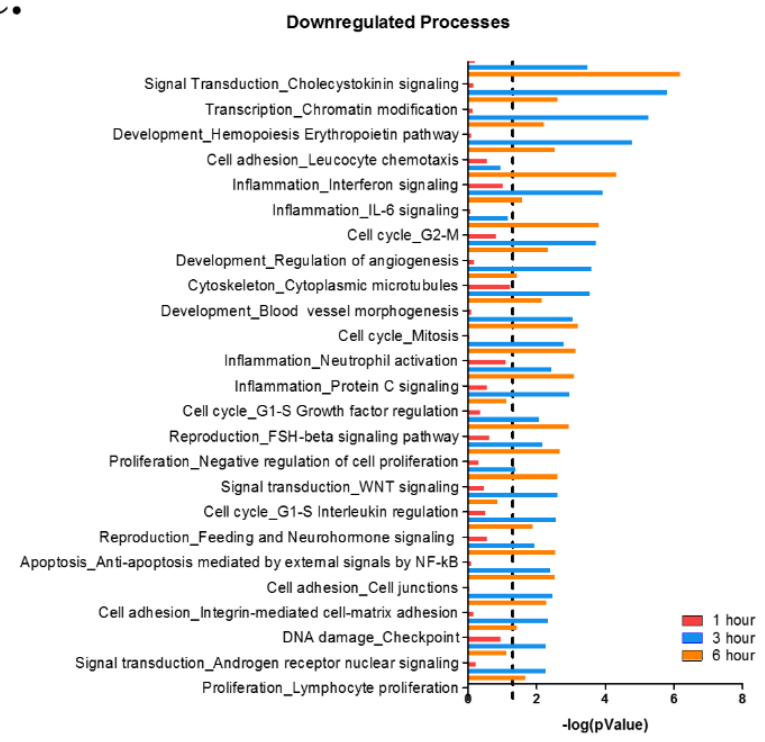

B.

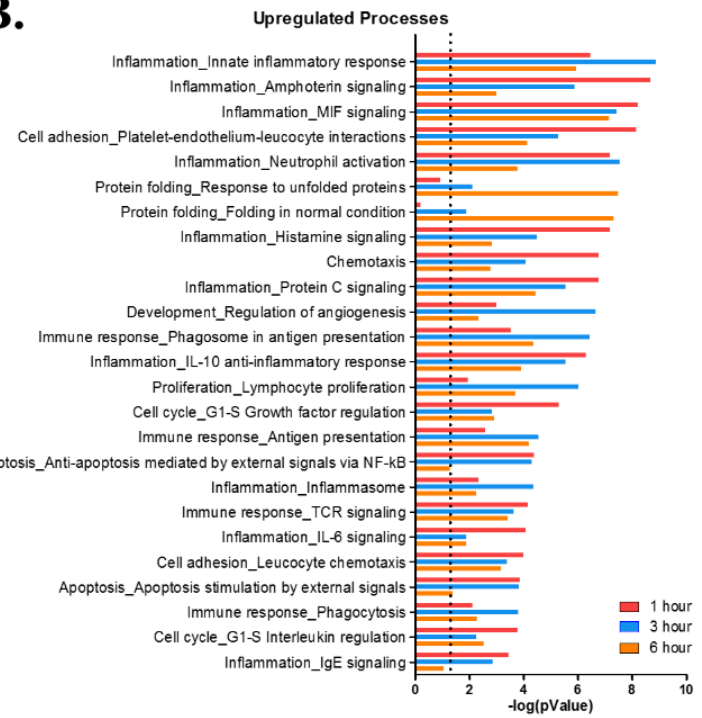

Figure 2.2: Biological processes affected during $F$. alocis challenge. Differentially expressed genes (DEG) for each timepoint were uploaded into the MetaCore database and analyzed for biological processes with a significant number of DEG. The frequency of each cell function was tallied for all the 71 significant $(\mathrm{p}<0.01)$ processes. The percentage of each function are displayed as a donut graph (A). The top 25 upregulated (B) or downregulated (C) network processes are graphed according to the - $\log$ of the $\mathrm{p}$ value for each time point. The dotted line represents a $\mathrm{p}$ value of 0.01 . 


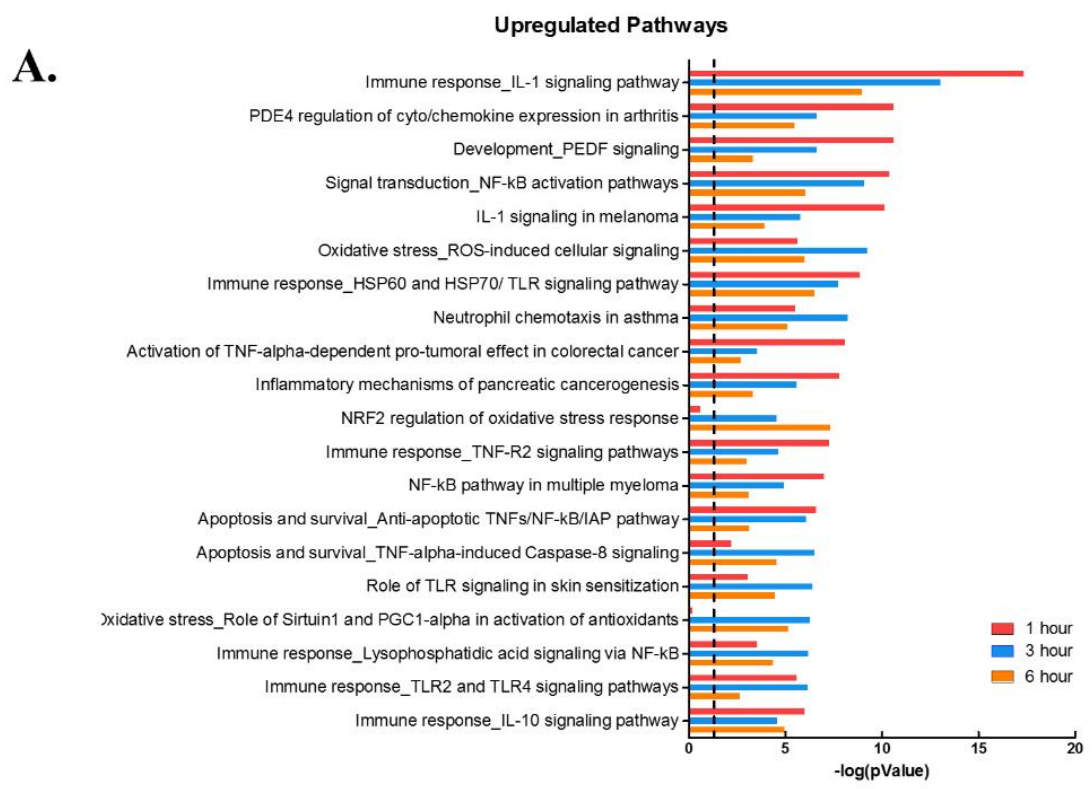

B. Dowregulated Pathways

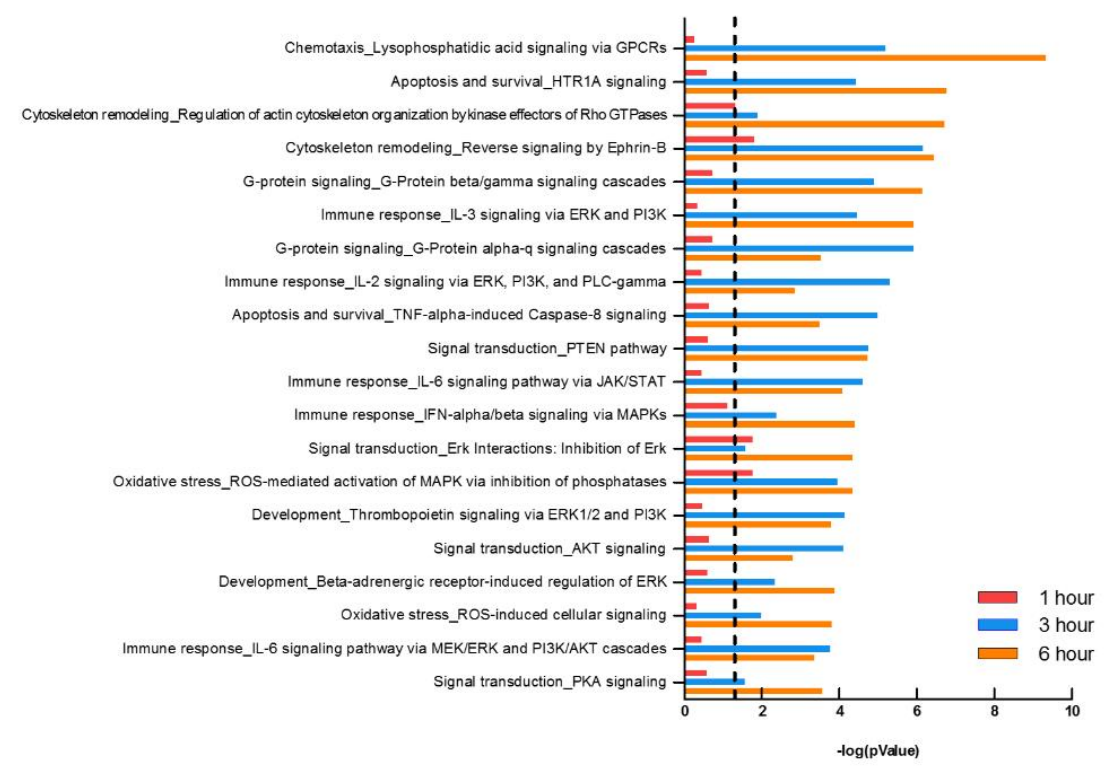

Figure 2.3: Pathways affected during $F$. alocis challenge. Using the MetaCore database, the top 20 upregulated (A) or downregulated (B) pathways in the transcriptome of $F$. alocis-challenged neutrophils were determined. These pathways are graphed according to the $-\log 10$ of the $\mathrm{p}$ value for each time point. The dotted line represents a $\mathrm{p}$ value of 0.01 . 


\begin{tabular}{|c|c|c|c|c|c|c|c|c|}
\hline & \multicolumn{2}{|c|}{$1 \mathrm{~h}$} & \multicolumn{2}{|c|}{$3 \mathrm{~h}$} & \multicolumn{2}{|c|}{$6 \mathrm{~h}$} & \multicolumn{2}{|c|}{$10 \mathrm{~h}$} \\
\hline & Media & F. alocis & Media & F. alocis & Media & $F$. alocis & Media & F. alocis \\
\hline fMLF & $-\quad+$ & + & + & + & $-\quad+$ & $-\quad+$ & $-\quad+$ & + \\
\hline Phospho-ERK & $=$ & $=$ & $=$ & $=$ & $=$ & $=$ & - & $-=$ \\
\hline Total ERK & $-=$ & $=$ & $=$ & $=$ & -- & $=$ & - & -- \\
\hline Phospho-p38 & - & - & -- & -- & - & -- & -- & -- \\
\hline Total p38 & $-m$ & -- & $=$ & -1 & -- & $-=$ & $-\cdots$ & -- \\
\hline
\end{tabular}

B.

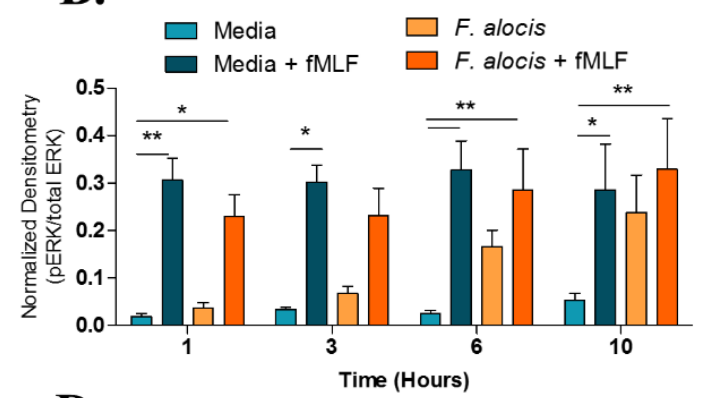

D.
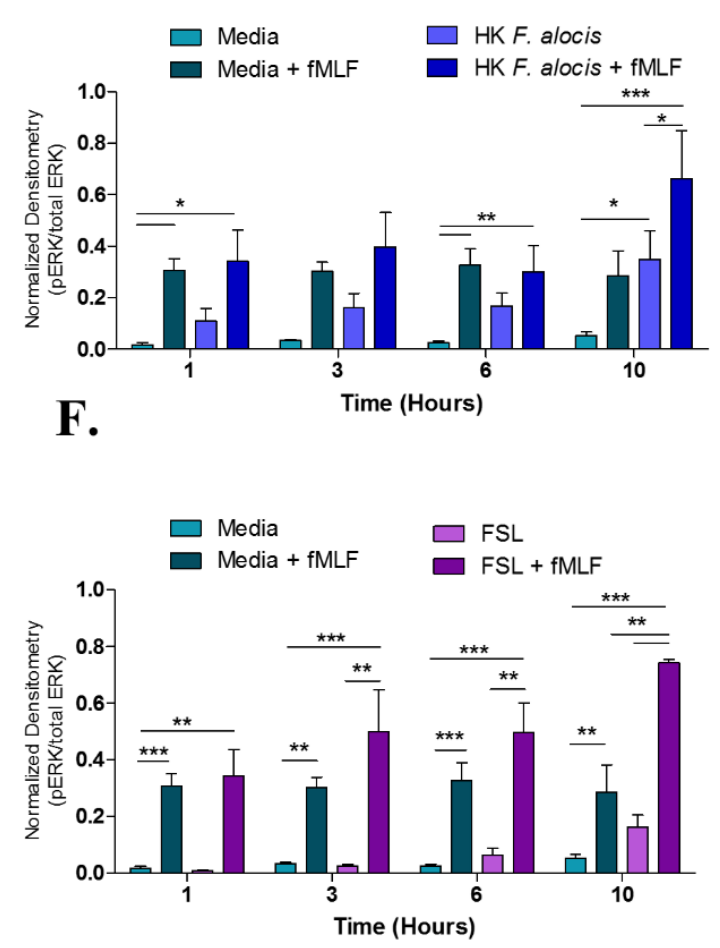

C.

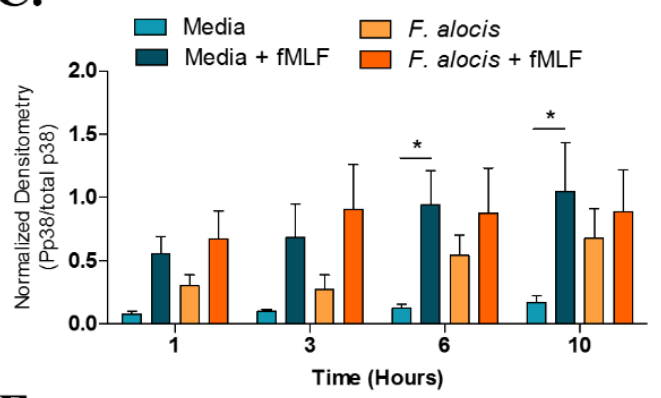

E.
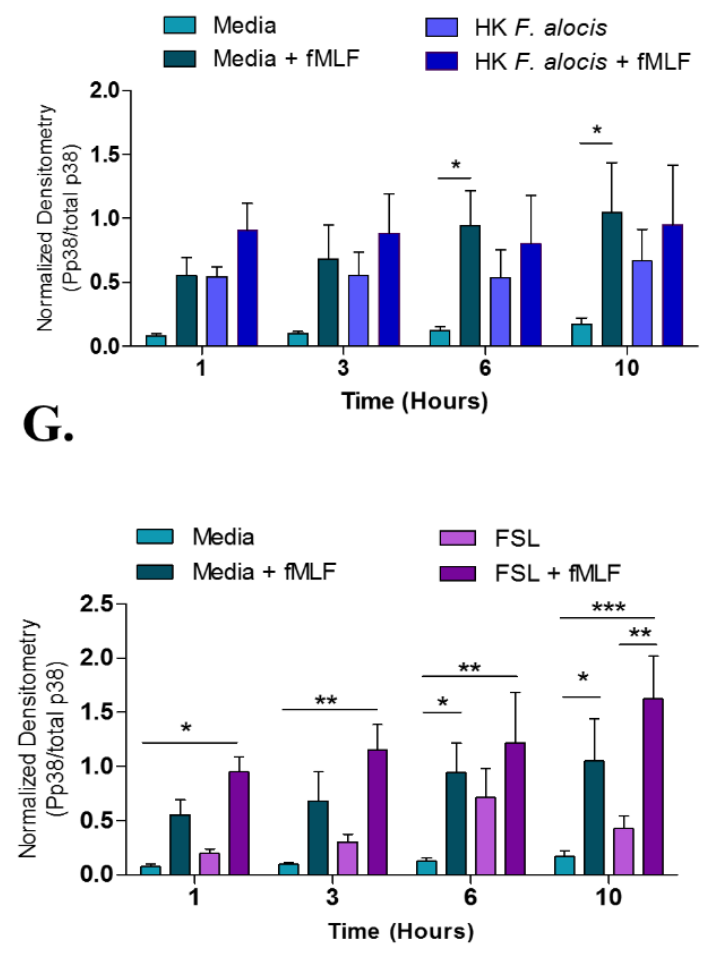

Figure 2.4: $F$. alocis effect on fMLF-induced MAPK activation 
Figure 2.4: To assess whether $F$. alocis is interfering with MAPK signaling, human neutrophils were cultured in media with or without $F$. alocis at an MOI of 10 for 1, 3, 6, or 10 hours followed by stimulation with fMLF for $1 \mathrm{~min}$. Western blots of whole cell lysates were probed for phosphorylated and total p38 and ERK1/2 (A) and quantified by densitometry $(\mathrm{B}, \mathrm{C})$. Alternatively, neutrophils were cultured in media alone, media with heat-killed $F$. alocis (D, E) or the TLR2/6 agonist FSL (F, G) for 1, 3, 6, or 10 hours before fMLF stimulation. Densitometries are plotted as the mean \pm SEM from 6 (B, C) and 4 (DG) independent experiments. Statistical differences among experimental conditions and time points were analyzed by a repeating measures two-way ANOVA, followed by Bonferroni post-tests. $*=\mathrm{p}<0.05, * *=\mathrm{p}<0.01, * * *=\mathrm{p}<0.001$. 

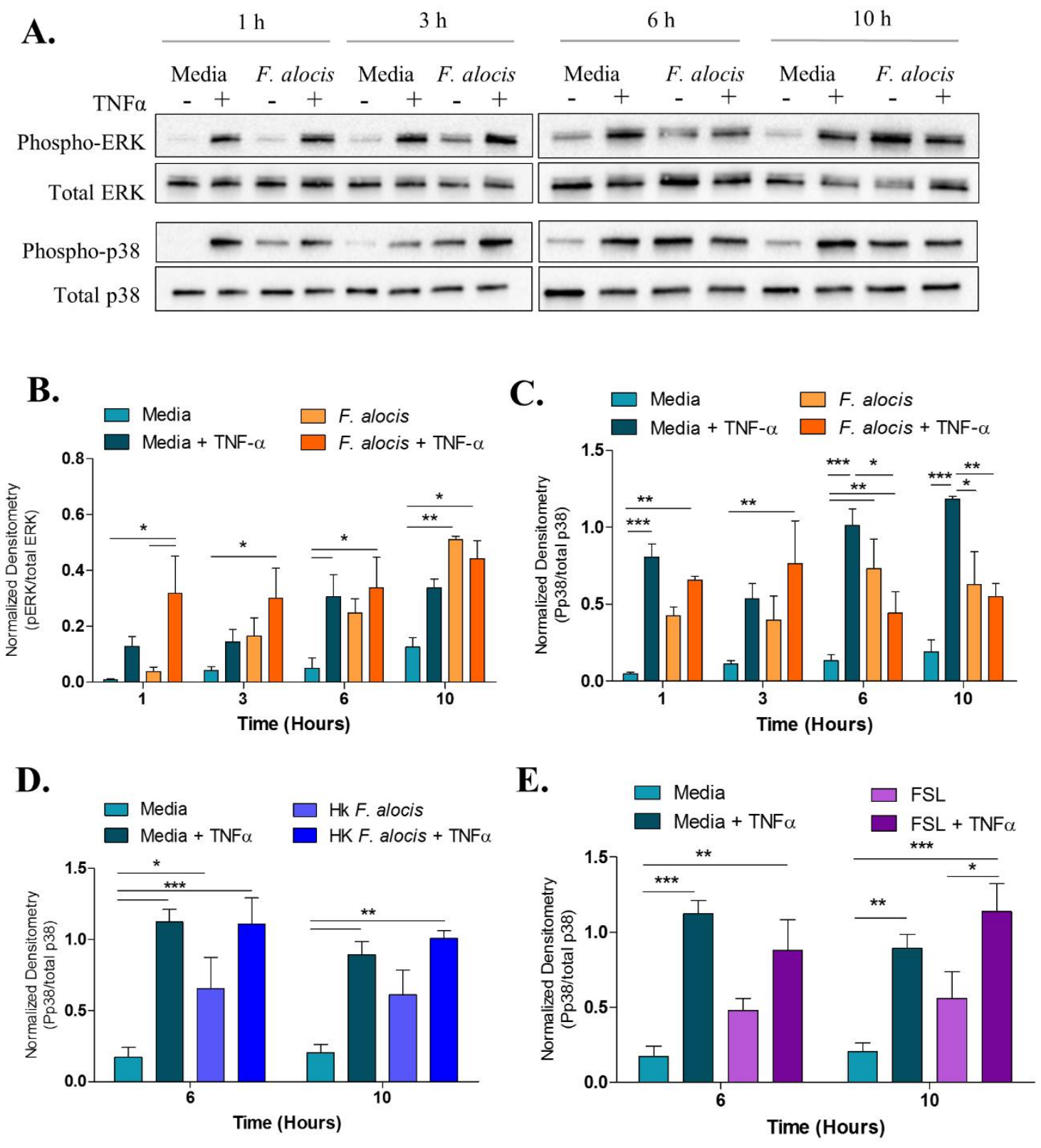

Figure 2.5: $F$. alocis effect on TNFa-induced MAPK activation 
Figure 2.5: Human neutrophils were cultured in media with or without $F$. alocis at an MOI of 10 for $1,3,6$, or 10 hours followed by stimulation with TNF- $\alpha$ for $15 \mathrm{~min}$. Western blots with whole cell lysates were probed for phosphorylated and total p38 and ERK1/2 (A) and quantified by densitometry $(\mathrm{B}, \mathrm{C})$. To determine if the decreased p38 phosphorylation at 6 and 10 hours was specific to the viable bacteria or a consequence of TLR 2/6 ligation, neutrophils were cultured in media alone, media with heat-killed $F$. alocis (D) or the TLR2/6 agonist FSL (E) for 6 or 10 hours before TNF- $\alpha$ stimulation. Western blots of whole cell lysates were probed for phosphorylated and total p38. Densitometries are plotted as the mean \pm SEM from 3 independent experiments. Statistical differences among experimental conditions and time points were analyzed by a repeating measures two-way ANOVA, followed by Bonferroni post-tests. $*=\mathrm{p}<0.05, * *=\mathrm{p}<0.01, * * *=\mathrm{p}<0.001$. 

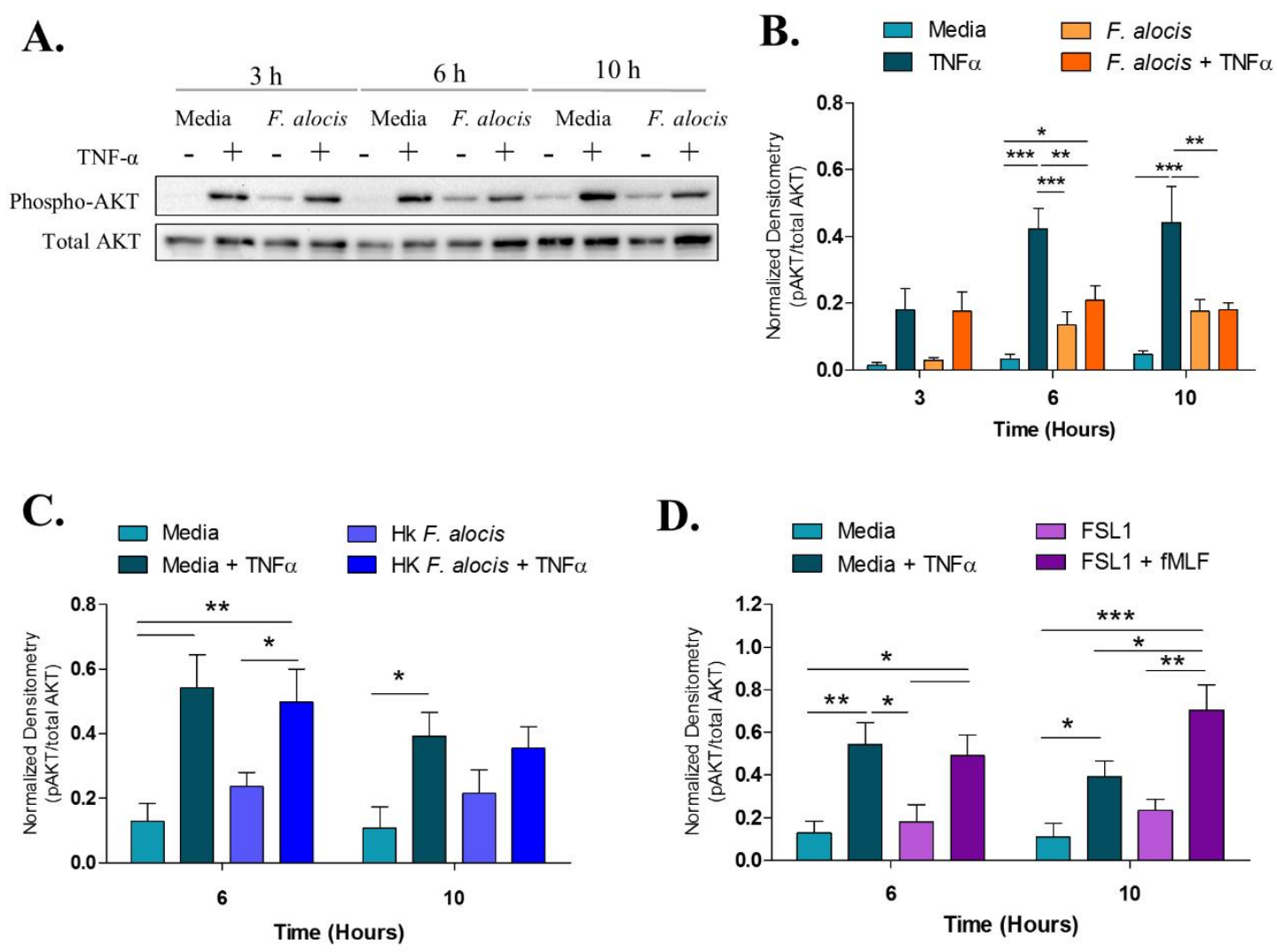

Figure 2.6: $F$. alocis effect on TNFa-induced AKT activation. Whole cell lysates from human neutrophils pre-treated with media alone or F. alocis (MOI 10) for 3, 6, or 10 hours followed by TNF- $\alpha$ stimulation were probed for phosphorylated and total AKT (A) and quantified by densitometry (B). Similarly, neutrophils were cultured in media alone, media with heat-killed F. alocis (C) or the TLR2/6 agonist FSL (D) for 6 or 10 hours before TNF$\alpha$ stimulation. Densitometries are plotted as the mean \pm SEM from 3 (B) and 4 (C, D) independent experiments. Statistical differences among experimental conditions and time points were analyzed by a repeating measures two-way ANOVA, followed by Bonferroni post-tests. $*=\mathrm{p}<0.05, * *=\mathrm{p}<0.01, * * *=\mathrm{p}<0.001$. 

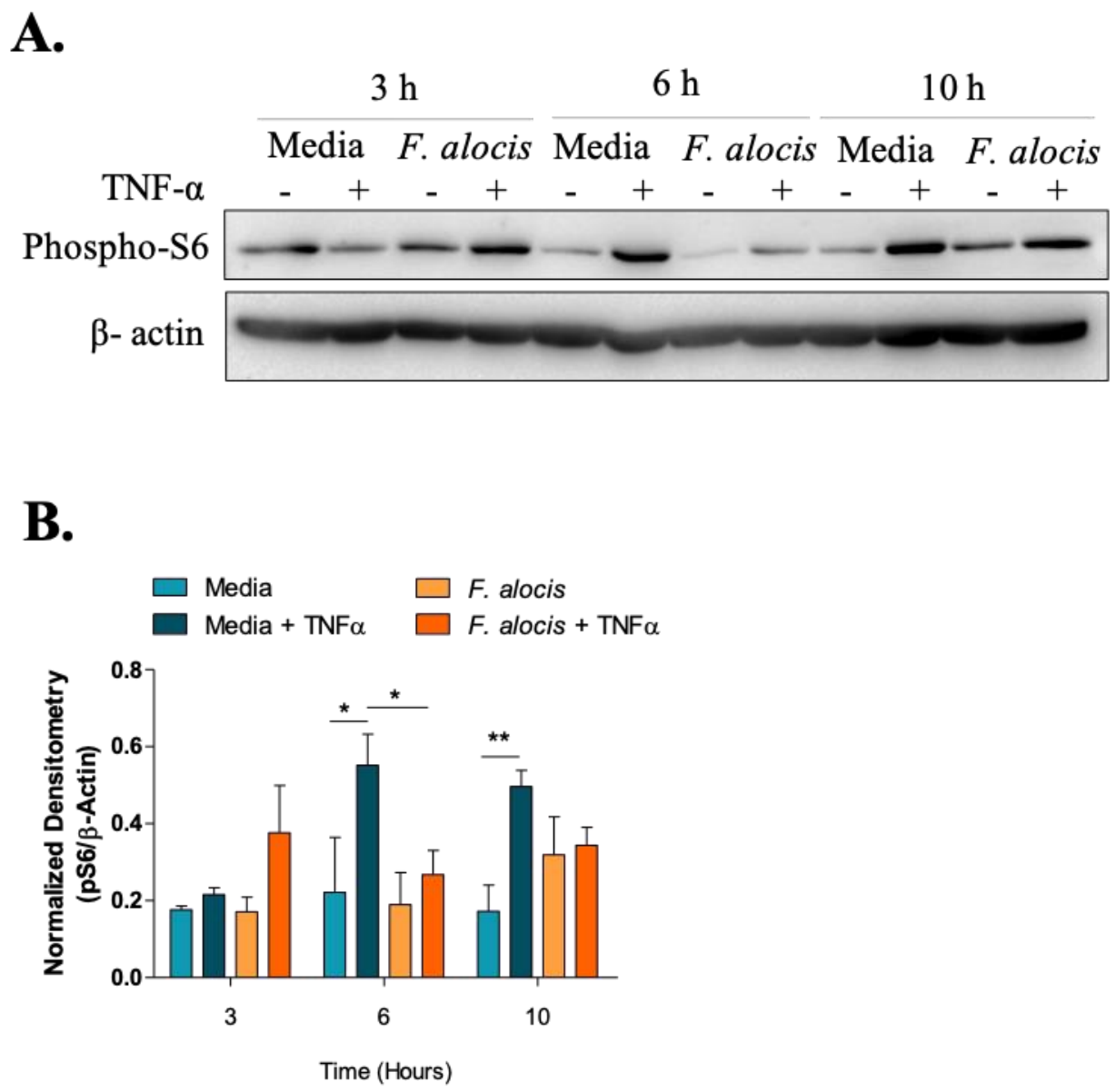

Figure 2.7: Dampened p38 MAPK phosphorylation affects the activation of translation machinery. Western blots for S6 ribosomal protein, S6, were performed on whole cell lysates from human neutrophils cultured in media or with $F$. alocis for 3, 6, and $10 \mathrm{hrs}$, followed by no stimulation or TNF $\alpha$ stimulation for $15 \mathrm{~min}$ (A). The protein expression of phosphorylated S6 was quantified by densitometry, normalized to $\beta$-actin, and plotted as the mean \pm SEM from three independent experiments (B). 
A.

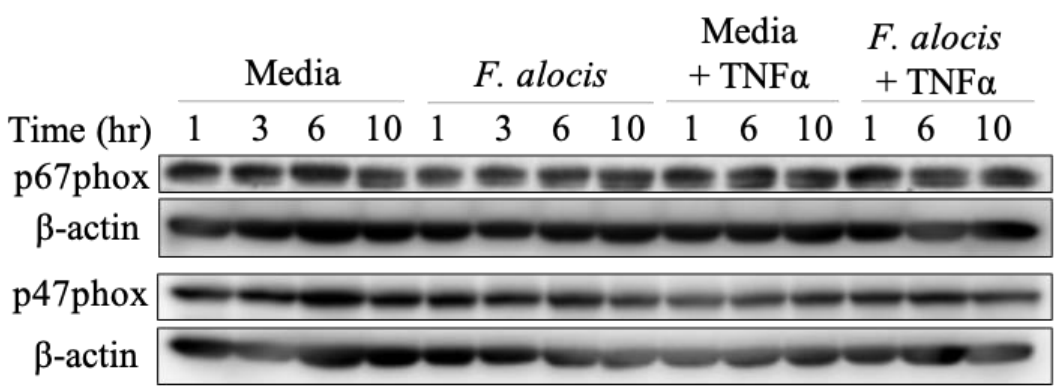

B.

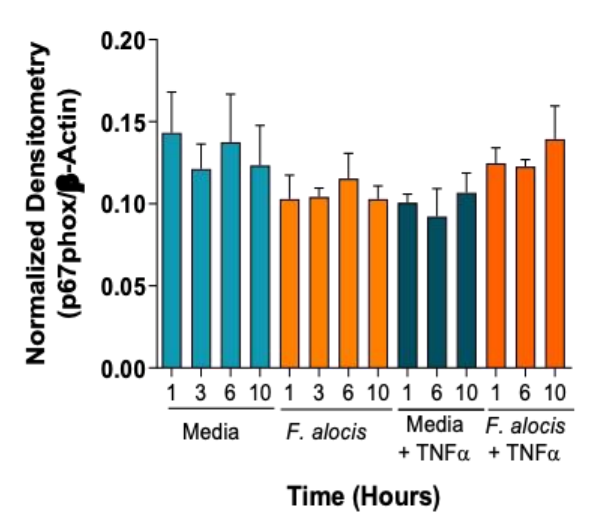

C.

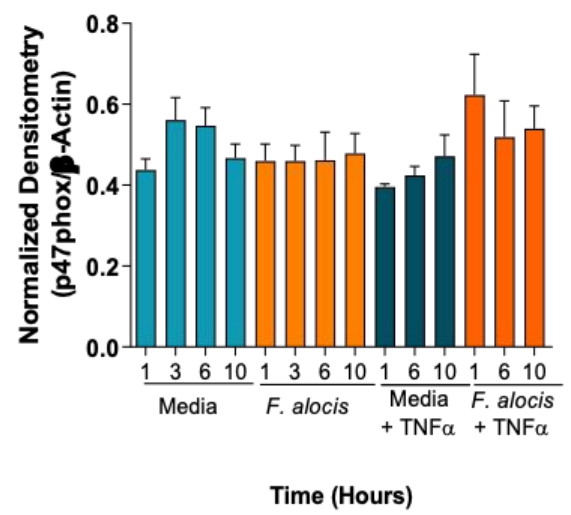

Figure 2.8: $F$. alocis challenge has no effect on the protein expression of NADPH oxidase components. Western blots for NADPH oxidase components, p67phox and p47phox, were performed on whole cell lysates from human neutrophils cultured in media or with $F$. alocis for 1, 3, 6, and $10 \mathrm{hrs,} \mathrm{followed} \mathrm{by} \mathrm{no} \mathrm{stimulation} \mathrm{or} \mathrm{TNF} \alpha$ stimulation for $15 \min (\mathrm{A})$. The protein expression of p67phox (B) and p47phox (C) was quantified by densitometry, normalized to $\beta$-actin, and plotted as the mean \pm SEM from three independent experiments. 
A.

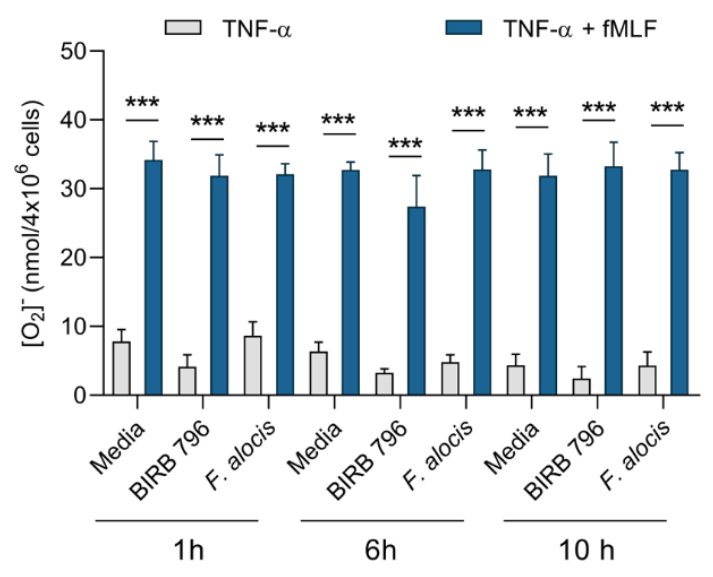

C.

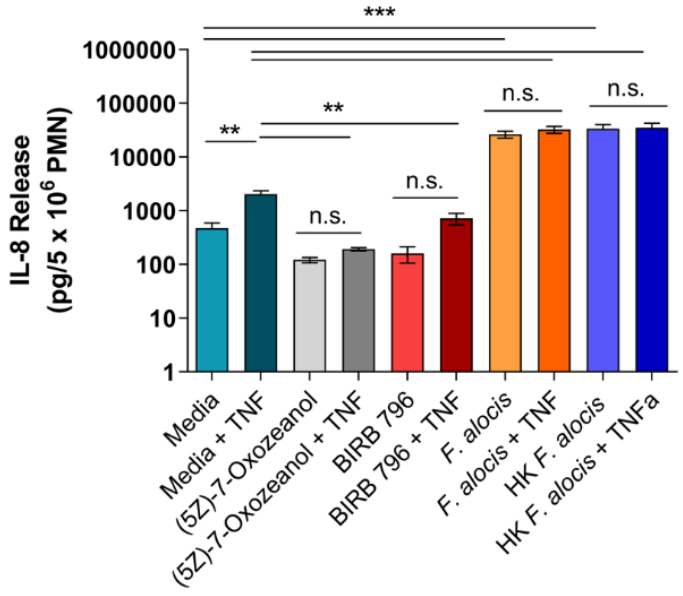

B.

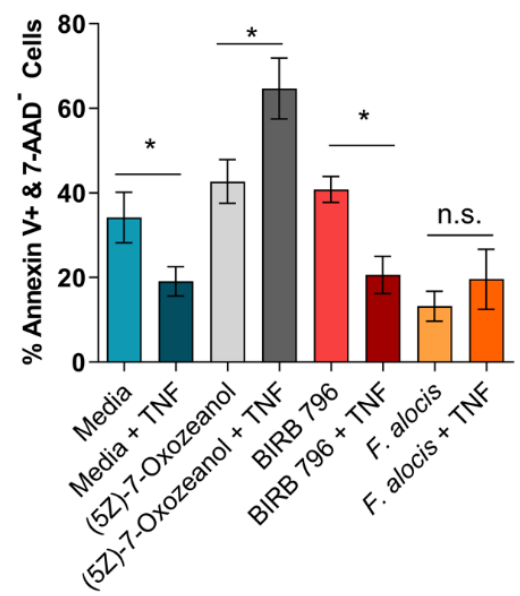

D.

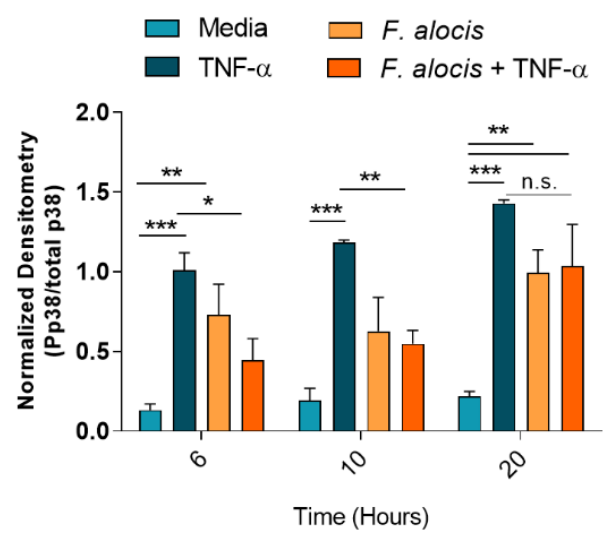

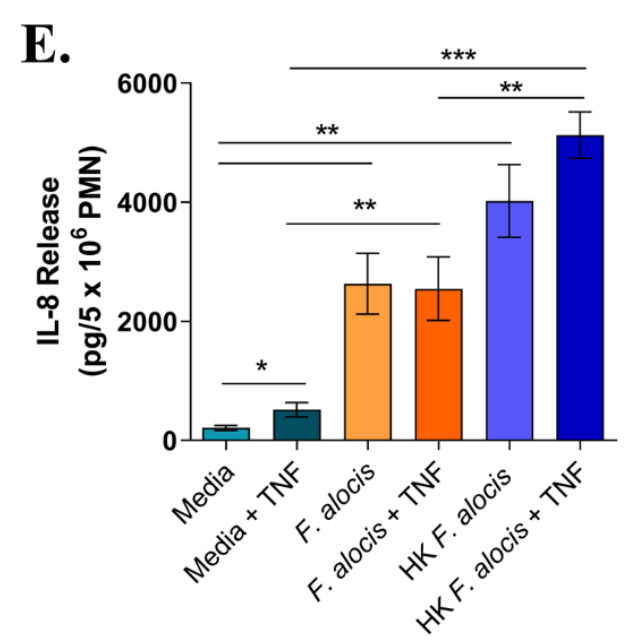

Figure 2.9: $F$. alocis effect on TNFa-induced functional responses 
Figure 2.9: The ability of $\mathrm{TNF} \alpha$ to prime media, BIRB 796 or $F$. alocis pre-treated neutrophils was assessed as the production of superoxide release (A). Cells were cultured in media, pre-treated with $F$. alocis for 1, 6, and 10 hours, or the p38 inhibitor BIRB 796 (60 min) followed by $\mathrm{TNF} \alpha$ alone or $\mathrm{TNF} \alpha+$ fMLF. Results from three independent experiments are shown as the mean \pm SEM superoxide production from basal TNF $\alpha$ stimulation or from cells primed with TNFa followed by fMLF stimulation. To test apoptosis, neutrophils were cultured in media or pre-treated with the TAK1 inhibitor (5Z)7-Oxozeanol (30 min), BIRB-796 (60 min), or F. alocis for 6 hours followed by a 12-hour TNF $\alpha$ stimulation (B). The apoptosis data are plotted as the mean \pm SEM percent of early apoptotic cells (Q3: Annexin V+, 7-AAD-) from three independent experiments. IL-8 production was also tested in neutrophils described above as well as neutrophils pre-treated with heat-killed $F$. alocis for 6 hours prior to a 12-hour stimulation with TNF $\alpha(C)$. The cytokine data are graphed as the mean \pm SEM IL-8 release from 5 independent experiments. The duration of $F$. alocis' inhibitory effect on TNF $\alpha$-mediated p38 phosphorylation was tested at 6, 10, and 20 hours (D). Normalized western blot densitometries are summarized from 3 independent experiments. Finally, IL-8 production was also tested in neutrophils cultured in media or pre-treated with live or heat-killed $F$. alocis for 6 hours prior to a 4-hour stimulation with TNFa (E). Data is graphed as the mean \pm SEM IL-8 release from 4 independent experiments. N.s. $=$ no significance, $*=p<0.05$ $* *=\mathrm{p}<0.01, * * *=\mathrm{p}<0.001$. 


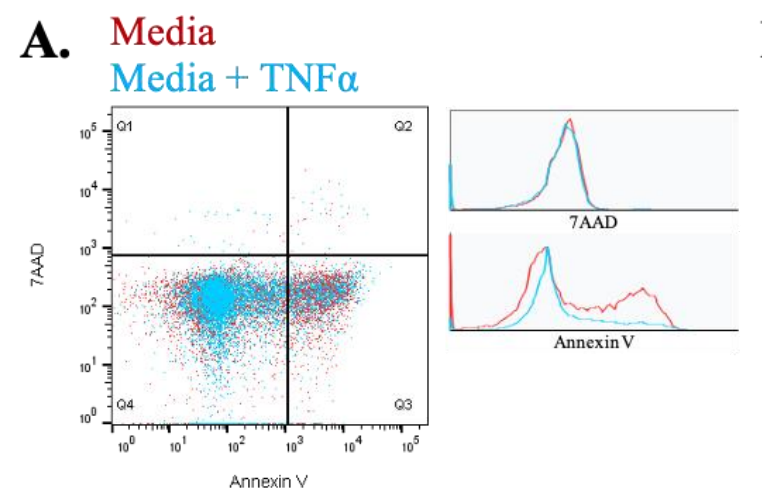

B. (5Z)-7-Oxozeanol

(5Z)-7-Oxozeanol+ TNF $\alpha$
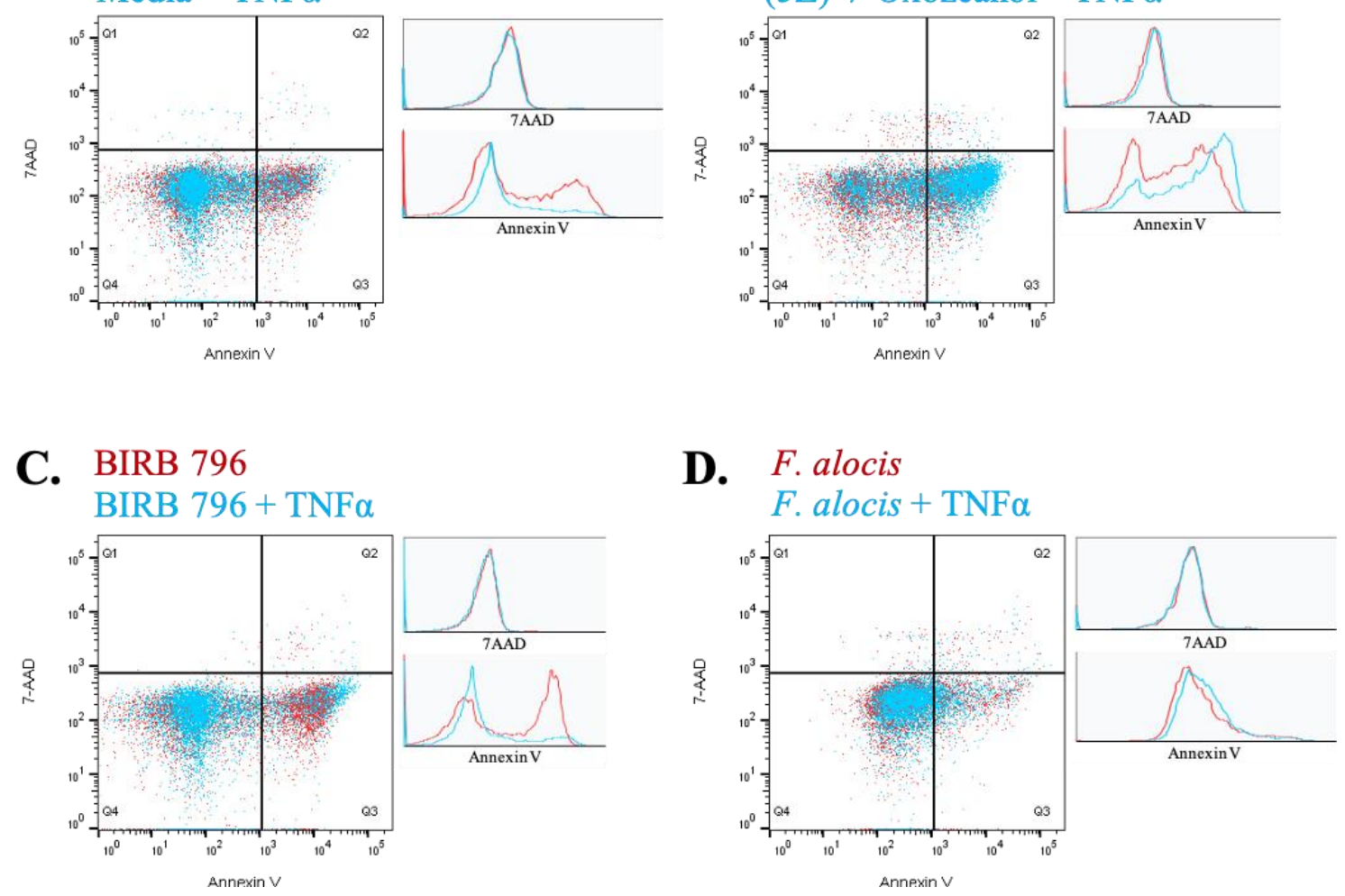

Figure 2.10: BIRB-796 and 6-hour $F$. alocis treatment do not inhibit TNFa prosurvival effect. Neutrophils were cultured in media (A) or media with TAK 1 inhibitor (5Z)-7-Oxozeanol (B), p38 inhibitor BIRB-796 (C), or F. alocis MOI 10 (D) for 6 hours, followed by +/- TNF $\alpha$ stimulation for 12 hours. Cells were stained for Annexin V and 7$\mathrm{AAD}$ and apoptosis was determined by flow cytometry. Flow cytometry scatter plots show the cells that did not receive TNF $\alpha$ (red) and those that did (blue). Histograms for each marker are shown to the side. 
A. Media

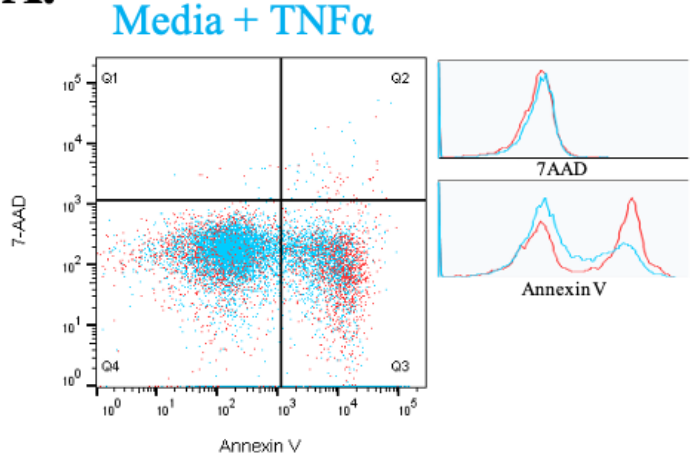

C. BIRB 796

C. BIRB $796+\mathrm{TNF} \alpha$

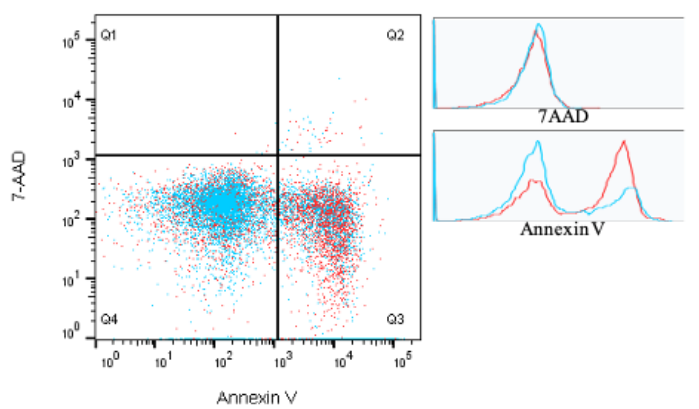

B. (5Z)-7-Oxozeanol (5Z)-7-Oxozeanol $+\mathrm{TNF} \alpha$

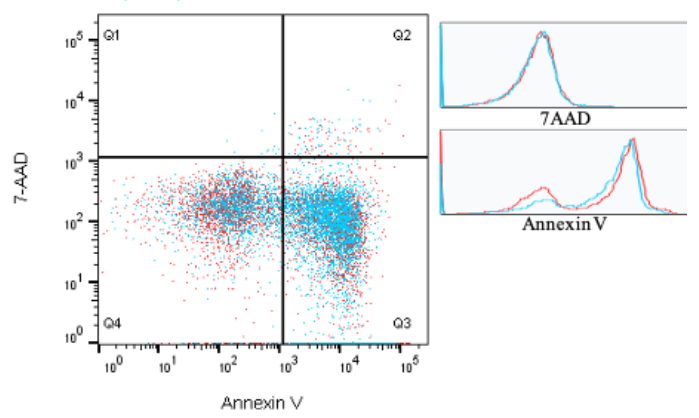

D. F. alocis $\mathrm{F}$ alocis $+\mathrm{TNF} \alpha$

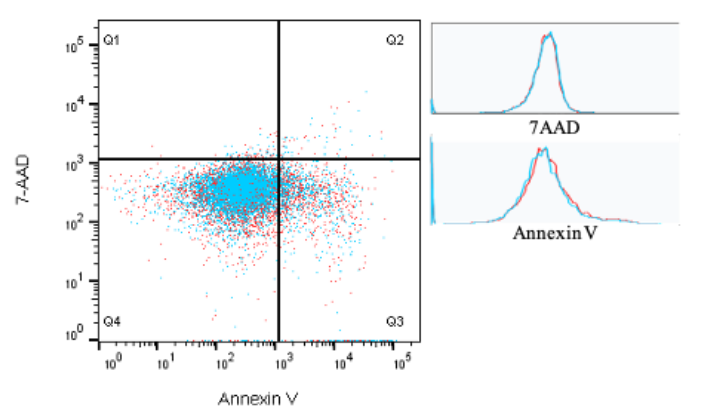

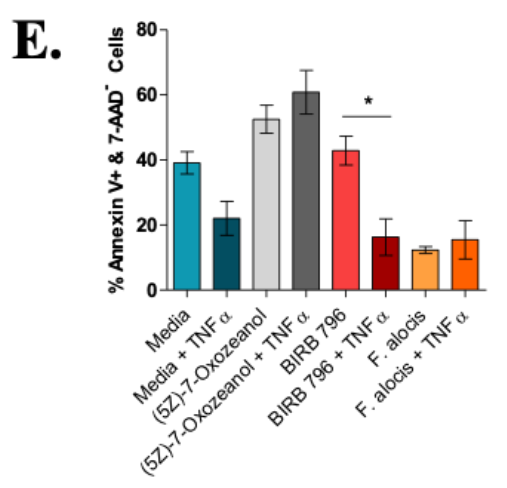

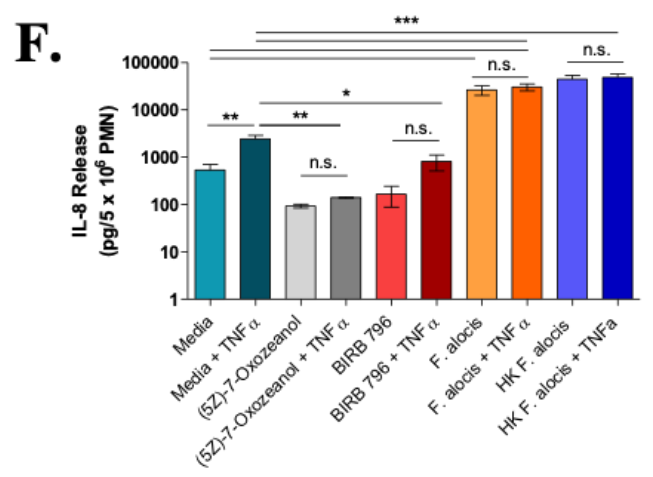

Figure. 2.11: BIRB-796 and 10-hour $F$. alocis treatments do not inhibit the TNFa functional effects. 
Fig. 2.11: The apoptotic status was tested in neutrophils cultured in media (A), media with TAK 1 inhibitor (5Z)-7-Oxozeanol (B), p38 inhibitor BIRB-796 (C), or F. alocis MOI 10 (D) for 10 hours, followed by +/- TNF $\alpha$ stimulation for 12 hours. Cells were stained for Annexin V and 7-AAD and apoptosis was determined by flow cytometry. Flow cytometry scatter plots show the cells that did not receive $\mathrm{TNF} \alpha$ (red) and those that did (blue). Histograms with the mean fluorescence intensity for each marker are shown to the right of each plot. Data is summarized as the mean \pm SEM percent of early apoptotic cells (Q3) from three independent experiments $(\mathbf{E})$. The supernatants from these cells were tested for IL-8 production $(\mathbf{F})$. Data is graphed as the mean \pm SEM IL-8 release from 4 independent experiments. 
TNF- $\alpha$

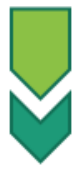

TNF Receptor (TNFR)

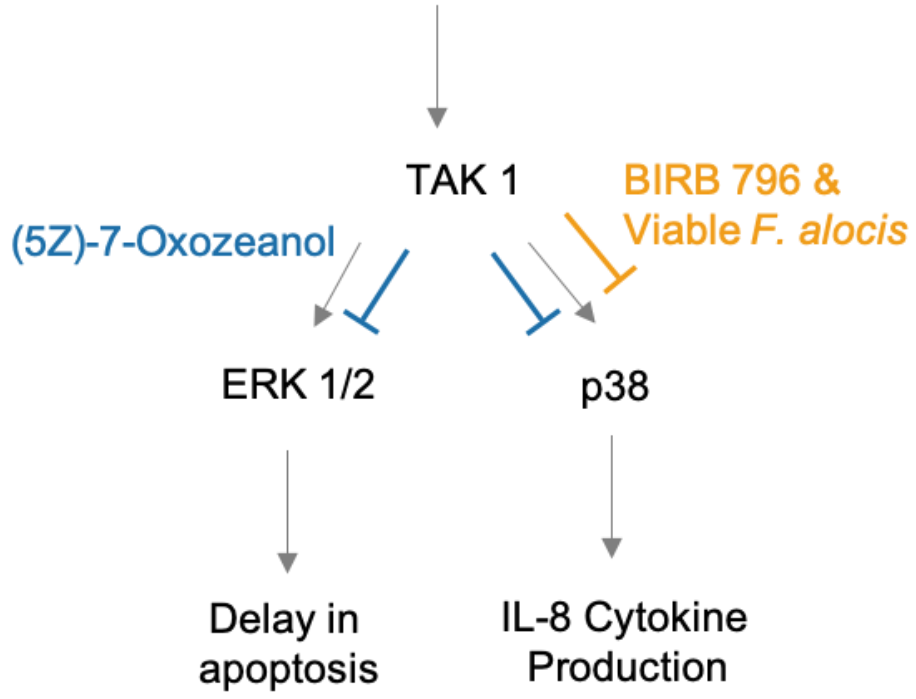

Figure 2.12: Proposed working model. Normal TNF $\alpha$ signaling pathway in neutrophils starts with ligation of TNFR and activation of TAK 1, which phosphorylates ERK1/2 to delay apoptosis and phosphorylates p38 to induce cytokine production. However, challenge of neutrophils with viable $F$. alocis results in decreased $\mathrm{p} 38$ activation by TNF $\alpha$. Ultimately, this results in decreased cytokine (IL-8) production, but does not interfere with the TNF $\alpha$-mediated delay in apoptosis. 
Table 2.1: qPCR primer sequences used to validate RNAseq results

\begin{tabular}{|c|lc|}
\hline \multirow{2}{*}{ Gene } & \multicolumn{2}{|l|}{ qPCR Primer Sequence } \\
\hline \multirow{2}{*}{ Galectin 3 } & Forward: & 5'-CAGAATTGCTTTAGATTTCCAA-3' \\
\cline { 2 - 3 } & Reverse: & 5'-TTATCCAGCTTTGTATTGCAA-3' \\
\hline \multirow{2}{*}{ NCF-1 } & Forward & 5'-AAGATGGCAAGAGTACCGC-3' \\
\cline { 2 - 3 } & Reverse & 5'-TCTCGTAGTTGGCAATGGC-3' \\
\hline \multirow{2}{*}{ GAPDH } & Forward & 5'-CTTTGGTATCGTGGAAGGACTC-3' \\
\cline { 2 - 3 } & Reverse & 5'-GTAGAGGCAGGGATGATGTTC-3' \\
\hline \multirow{2}{*}{ CXCL5 } & Forward & 5'-TCTGCAAGTGTTCGCCATAG-3' \\
\cline { 2 - 3 } & Reverse & 5'-CAGTTTCCTTGTTTCCACCG-3' \\
\hline \multirow{2}{*}{ CCL5 } & Forward & 5'-TGCCCACATCAAGGAGTATTT-3' \\
\cline { 2 - 3 } & Reverse & 5'-TTTCGGGTGACAAAGACGA-3' \\
\hline \multirow{2}{*}{ ASC } & Forward & 5'-CTCCTCAGTCGGCAGCCAAG-3' \\
\cline { 2 - 3 } & Reverse & 5'-ACAGAGCATCCAGCAGCCAC-3' \\
\hline \multirow{2}{*}{ NOD2 } & Forward & 5'-CTGAAGAATGCCCGCAAGGT-3' \\
\cline { 2 - 3 } & Reverse & 5'-GTCTCTTGGAGCAGGCGGATG-3' \\
\hline
\end{tabular}


Table 2.2: Significant biological processes from DAVID analysis categorized by cell function

\begin{tabular}{|c|c|c|c|c|c|c|}
\hline \multirow{7}{*}{ 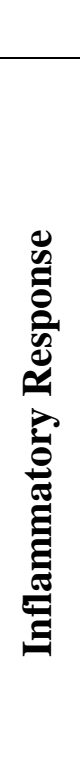 } & \multicolumn{2}{|l|}{$\underline{1 \text { Hour }}$} & \multicolumn{2}{|l|}{$\underline{3 \text { Hour }}$} & \multicolumn{2}{|l|}{6 Hour } \\
\hline & Process & P-value & Process & $\begin{array}{l}\text { P- } \\
\text { value }\end{array}$ & Process & $\begin{array}{l}P \text { P- } \\
\text { value }\end{array}$ \\
\hline & inflammatory response & $1.40 \mathrm{E}-13$ & $\begin{array}{l}\text { inflammatory } \\
\text { response }\end{array}$ & $\begin{array}{l}3.30 \mathrm{E} \\
-15\end{array}$ & inflammatory response & $\begin{array}{l}4.80 \mathrm{E} \\
-09\end{array}$ \\
\hline & immune response & $7.30 \mathrm{E}-08$ & $\begin{array}{l}\text { regulation of } \\
\text { inflammatory } \\
\text { response }\end{array}$ & $\begin{array}{l}1.10 \mathrm{E} \\
-04\end{array}$ & innate immune response & $\begin{array}{l}8.50 \mathrm{E} \\
-06\end{array}$ \\
\hline & $\begin{array}{l}\text { positive regulation of } \\
\text { inflammatory response }\end{array}$ & $9.30 \mathrm{E}-04$ & $\begin{array}{l}\text { positive regulation of } \\
\text { inflammatory } \\
\text { response }\end{array}$ & $\begin{array}{l}5.50 \mathrm{E} \\
-04\end{array}$ & $\begin{array}{l}\text { negative regulation of } \\
\text { inflammatory response }\end{array}$ & $\begin{array}{l}1.50 \mathrm{E} \\
-03\end{array}$ \\
\hline & $\begin{array}{l}\text { negative regulation of } \\
\text { inflammatory response }\end{array}$ & $9.00 \mathrm{E}-03$ & $\begin{array}{l}\text { negative regulation of } \\
\text { inflammatory } \\
\text { response }\end{array}$ & $\begin{array}{l}3.60 \mathrm{E} \\
-03\end{array}$ & $\begin{array}{l}\text { positive regulation of } \\
\text { inflammatory response }\end{array}$ & $\begin{array}{l}1.60 \mathrm{E} \\
-03\end{array}$ \\
\hline & & & immune response & $\begin{array}{l}6.70 \mathrm{E} \\
-03\end{array}$ & $\begin{array}{l}\text { regulation of } \\
\text { inflammatory response }\end{array}$ & $\begin{array}{l}1.50 \mathrm{E} \\
-02\end{array}$ \\
\hline & Process & P-value & Process & $\begin{array}{l}\text { P- } \\
\text { value }\end{array}$ & Process & $\begin{array}{l}\text { P- } \\
\text { value }\end{array}$ \\
\hline$\frac{\mathscr{U}_{0}^{2}}{0}$ & $\begin{array}{l}\text { cellular response to } \\
\text { lipopolysaccharide }\end{array}$ & $1.70 \mathrm{E}-04$ & transferrin transport & $\begin{array}{l}2.90 \mathrm{E} \\
-06\end{array}$ & $\begin{array}{l}\text { response to } \\
\text { lipopolysaccharide }\end{array}$ & $\begin{array}{l}1.30 \mathrm{E} \\
-03\end{array}$ \\
\hline$\underset{0}{0}$ & $\begin{array}{l}\text { response to } \\
\text { lipopolysaccharide }\end{array}$ & $1.60 \mathrm{E}-03$ & $\begin{array}{l}\text { response to } \\
\text { lipopolysaccharide }\end{array}$ & $\begin{array}{l}3.60 \mathrm{E} \\
-05\end{array}$ & $\begin{array}{l}\text { response to muramyl } \\
\text { dipeptide }\end{array}$ & $\begin{array}{l}3.80 \mathrm{E} \\
-03\end{array}$ \\
\hline$\stackrel{2}{0}$ & & & $\begin{array}{l}\text { lipopolysaccharide- } \\
\text { mediated signaling } \\
\text { pathway }\end{array}$ & $\begin{array}{l}4.90 \mathrm{E} \\
-05\end{array}$ & defense response & $\begin{array}{l}1.10 \mathrm{E} \\
-02\end{array}$ \\
\hline
\end{tabular}




\begin{tabular}{|c|c|c|c|c|c|c|}
\hline & \multicolumn{2}{|c|}{$\underline{1 \text { Hour }}$} & \multicolumn{2}{|l|}{ 3 Hour } & \multicolumn{2}{|l|}{6 Hour } \\
\hline & Process & P-value & Process & $\begin{array}{c}\text { P- } \\
\text { value }\end{array}$ & Process & $\begin{array}{c}\mathrm{P}- \\
\text { value }\end{array}$ \\
\hline \multirow{2}{*}{ 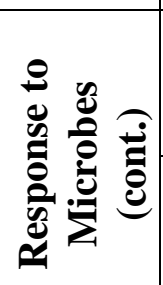 } & & & $\begin{array}{l}\text { cellular response to } \\
\text { lipopolysaccharide }\end{array}$ & $\begin{array}{l}3.70 \mathrm{E} \\
-04\end{array}$ & $\begin{array}{l}\text { lipopolysaccharide- } \\
\text { mediated signaling } \\
\text { pathway }\end{array}$ & $\begin{array}{l}1.80 \mathrm{E} \\
-02\end{array}$ \\
\hline & & & $\begin{array}{l}\text { response to muramyl } \\
\text { dipeptide }\end{array}$ & $\begin{array}{l}5.60 \mathrm{E} \\
-03\end{array}$ & $\begin{array}{l}\text { cellular response to } \\
\text { lipopolysaccharide }\end{array}$ & $\begin{array}{l}1.70 \mathrm{E} \\
-02\end{array}$ \\
\hline \multirow{10}{*}{ 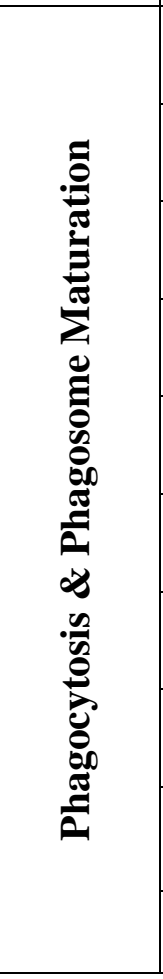 } & & & $\begin{array}{l}\text { cellular response to } \\
\text { oxidative stress }\end{array}$ & $\begin{array}{l}1.50 \mathrm{E} \\
-06\end{array}$ & $\begin{array}{l}\text { actin cytoskeleton } \\
\text { organization }\end{array}$ & $\begin{array}{l}1.10 \mathrm{E} \\
-02\end{array}$ \\
\hline & & & $\begin{array}{l}\text { phagosome } \\
\text { acidification }\end{array}$ & $\begin{array}{l}8.80 \mathrm{E} \\
-06\end{array}$ & phagocytosis & $\begin{array}{l}4.00 \mathrm{E} \\
-03\end{array}$ \\
\hline & & & $\begin{array}{l}\text { intracellular protein } \\
\text { transport }\end{array}$ & $\begin{array}{l}3.20 \mathrm{E} \\
-04\end{array}$ & phagosome acidification & $\begin{array}{l}6.00 \mathrm{E} \\
-03\end{array}$ \\
\hline & & & $\begin{array}{l}\text { response to hydrogen } \\
\text { peroxide }\end{array}$ & $\begin{array}{l}7.70 \mathrm{E} \\
-04 \\
\end{array}$ & $\begin{array}{l}\text { response to hydrogen } \\
\text { peroxide }\end{array}$ & $\begin{array}{l}7.30 \mathrm{E} \\
-04 \\
\end{array}$ \\
\hline & & & $\begin{array}{l}\text { regulation of } \\
\text { macroautophagy }\end{array}$ & $\begin{array}{l}8.60 \mathrm{E} \\
-04\end{array}$ & $\begin{array}{l}\text { response to nitrosative } \\
\text { stress }\end{array}$ & $\begin{array}{l}4.60 \mathrm{E} \\
-03\end{array}$ \\
\hline & & & macroautophagy & $\begin{array}{l}2.60 \mathrm{E} \\
-03\end{array}$ & $\begin{array}{l}\text { cellular response to } \\
\text { oxidative stress }\end{array}$ & $\begin{array}{l}7.20 \mathrm{E} \\
-03\end{array}$ \\
\hline & & & autophagy & $\begin{array}{l}2.80 \mathrm{E} \\
-03\end{array}$ & $\begin{array}{l}\text { positive regulation of } \\
\text { autophagy }\end{array}$ & $\begin{array}{l}8.30 \mathrm{E} \\
-03\end{array}$ \\
\hline & & & $\begin{array}{l}\text { endosome to } \\
\text { lysosome transport }\end{array}$ & $\begin{array}{l}5.40 \mathrm{E} \\
-03\end{array}$ & $\begin{array}{l}\text { positive regulation of } \\
\text { xenophagy }\end{array}$ & $\begin{array}{l}1.10 \mathrm{E} \\
-02\end{array}$ \\
\hline & & & & & $\begin{array}{l}\text { negative regulation of } \\
\text { inclusion body assembly }\end{array}$ & $\begin{array}{l}1.60 \mathrm{E} \\
-02\end{array}$ \\
\hline & & & & & transferrin transport & $\begin{array}{l}7.80 \mathrm{E} \\
-04\end{array}$ \\
\hline
\end{tabular}




\begin{tabular}{|c|c|c|c|c|c|c|}
\hline & \multicolumn{2}{|l|}{$\underline{1 \text { Hour }}$} & \multicolumn{2}{|l|}{$\underline{3 \text { Hour }}$} & \multicolumn{2}{|l|}{$\underline{6 \text { Hour }}$} \\
\hline & Process & $\begin{array}{c}\text { P- } \\
\text { value }\end{array}$ & Process & $\begin{array}{c}\text { P- } \\
\text { value }\end{array}$ & Process & $\begin{array}{c}\text { P- } \\
\text { value }\end{array}$ \\
\hline \multirow{9}{*}{ 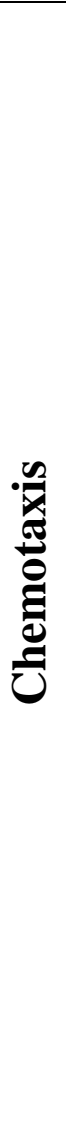 } & neutrophil chemotaxis & $\begin{array}{l}5.10 \mathrm{E}- \\
06\end{array}$ & neutrophil chemotaxis & $\begin{array}{l}\text { 4.70E- } \\
05\end{array}$ & leukocyte migration & $\begin{array}{l}\begin{array}{l}4.80 \mathrm{E}- \\
04\end{array} \\
\end{array}$ \\
\hline & monocyte chemotaxis & $\begin{array}{l}\text { 6.80E- } \\
05\end{array}$ & chemotaxis & $\begin{array}{l}1.30 \mathrm{E}- \\
04\end{array}$ & granulocyte chemotaxis & $\begin{array}{l}6.10 \mathrm{E}- \\
04\end{array}$ \\
\hline & lymphocyte chemotaxis & $\begin{array}{l}1.80 \mathrm{E}- \\
04\end{array}$ & $\begin{array}{l}\text { positive regulation of } \\
\text { neutrophil chemotaxis }\end{array}$ & $\begin{array}{l}6.00 \mathrm{E}- \\
04\end{array}$ & chemotaxis & $\begin{array}{l}2.50 \mathrm{E}- \\
03\end{array}$ \\
\hline & chemotaxis & $\begin{array}{l}2.70 \mathrm{E}- \\
04\end{array}$ & leukocyte migration & $\begin{array}{l}2.50 \mathrm{E}- \\
03\end{array}$ & neutrophil chemotaxis & $\begin{array}{l}3.80 \mathrm{E}- \\
03\end{array}$ \\
\hline & cell chemotaxis & $\begin{array}{l}5.50 \mathrm{E}- \\
04\end{array}$ & $\begin{array}{l}\text { positive regulation of cell } \\
\text { migration }\end{array}$ & $\begin{array}{l}5.90 \mathrm{E}- \\
03\end{array}$ & $\begin{array}{l}\text { positive regulation of } \\
\text { neutrophil chemotaxis }\end{array}$ & $\begin{array}{l}2.50 \mathrm{E}- \\
04\end{array}$ \\
\hline & $\begin{array}{l}\text { positive regulation of } \\
\text { neutrophil chemotaxis }\end{array}$ & $\begin{array}{l}1.40 \mathrm{E}- \\
03\end{array}$ & monocyte chemotaxis & $\begin{array}{l}8.60 \mathrm{E}- \\
03\end{array}$ & $\begin{array}{l}\text { positive regulation of cell } \\
\text { migration }\end{array}$ & $\begin{array}{l}1.10 \mathrm{E}- \\
03\end{array}$ \\
\hline & $\begin{array}{l}\text { positive regulation of cell } \\
\text { migration }\end{array}$ & $\begin{array}{l}3.00 \mathrm{E}- \\
03\end{array}$ & $\begin{array}{l}\text { small GTPase mediated } \\
\text { signal transduction }\end{array}$ & $\begin{array}{l}2.90 \mathrm{E}- \\
05\end{array}$ & $\begin{array}{l}\text { small GTPase mediated } \\
\text { signal transduction }\end{array}$ & $\begin{array}{l}3.10 \mathrm{E}- \\
05\end{array}$ \\
\hline & $\begin{array}{l}\text { positive regulation of } \\
\text { ERK1 and ERK } 2 \text { cascade }\end{array}$ & $\begin{array}{l}8.50 \mathrm{E}- \\
05\end{array}$ & $\begin{array}{l}\text { intracellular signal } \\
\text { transduction }\end{array}$ & $\begin{array}{l}5.70 \mathrm{E}- \\
04\end{array}$ & signal transduction & $\begin{array}{l}6.10 \mathrm{E}- \\
05\end{array}$ \\
\hline & $\begin{array}{l}\text { positive regulation of } \\
\text { peptidyl-serine } \\
\text { phosphorylation }\end{array}$ & $\begin{array}{c}\text { 7.70E- } \\
04\end{array}$ & & & & \\
\hline
\end{tabular}




\begin{tabular}{|c|c|c|c|c|c|c|}
\hline & \multicolumn{2}{|l|}{1 Hour } & \multicolumn{2}{|l|}{$\underline{3 \text { Hour }}$} & \multicolumn{2}{|l|}{6 Hour } \\
\hline & Process & $\begin{array}{c}\text { P- } \\
\text { value }\end{array}$ & Process & $\begin{array}{c}\text { P- } \\
\text { value }\end{array}$ & Process & $\begin{array}{c}\text { P- } \\
\text { value }\end{array}$ \\
\hline \multirow{9}{*}{ 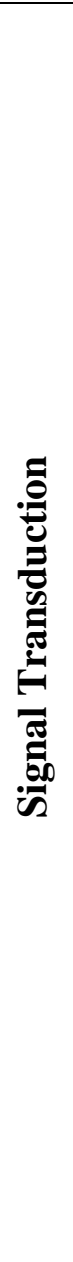 } & signal transduction & $\begin{array}{l}8.40 \mathrm{E}- \\
04\end{array}$ & $\begin{array}{l}\text { positive regulation of JNK } \\
\text { cascade }\end{array}$ & $\begin{array}{l}5.70 \mathrm{E}- \\
04\end{array}$ & $\begin{array}{l}\text { intracellular signal } \\
\text { transduction }\end{array}$ & $\begin{array}{l}3.70 \mathrm{E}- \\
03\end{array}$ \\
\hline & $\begin{array}{l}\text { G-protein coupled } \\
\text { receptor signaling } \\
\text { pathway }\end{array}$ & $\begin{array}{l}2.30 \mathrm{E}- \\
03\end{array}$ & signal transduction & $\begin{array}{l}1.10 \mathrm{E}- \\
03\end{array}$ & $\begin{array}{l}\text { positive regulation of } \\
\text { GTPase activity }\end{array}$ & $\begin{array}{l}4.30 \mathrm{E}- \\
03\end{array}$ \\
\hline & $\begin{array}{l}\text { activation of MAPKKK } \\
\text { activity }\end{array}$ & $\begin{array}{l}5.50 \mathrm{E}- \\
03\end{array}$ & $\begin{array}{l}\text { TRIF-dependent toll-like } \\
\text { receptor signaling } \\
\text { pathway }\end{array}$ & $\begin{array}{l}2.90 \mathrm{E}- \\
03\end{array}$ & $\begin{array}{l}\text { positive regulation of } \\
\text { ERK1 and ERK2 cascade }\end{array}$ & $\begin{array}{l}3.60 \mathrm{E}- \\
03\end{array}$ \\
\hline & $\begin{array}{l}\text { intracellular receptor } \\
\text { signaling pathway }\end{array}$ & $\begin{array}{l}7.00 \mathrm{E}- \\
03\end{array}$ & signaling & $\begin{array}{l}6.10 \mathrm{E}- \\
03\end{array}$ & $\begin{array}{l}\text { inactivation of MAPK } \\
\text { activity }\end{array}$ & $\begin{array}{l}1.40 \mathrm{E}- \\
02\end{array}$ \\
\hline & $\begin{array}{l}\text { positive regulation of } \\
\text { peptidyl-tyrosine } \\
\text { phosphorylation }\end{array}$ & $\begin{array}{l}1.00 \mathrm{E}- \\
02\end{array}$ & $\begin{array}{l}\text { regulation of protein } \\
\text { phosphorylation }\end{array}$ & $\begin{array}{l}8.90 \mathrm{E}- \\
03\end{array}$ & & \\
\hline & & & $\begin{array}{l}\text { inactivation of MAPK } \\
\text { activity }\end{array}$ & $\begin{array}{l}7.00 \mathrm{E}- \\
03\end{array}$ & & \\
\hline & & & $\begin{array}{l}\text { positive regulation of } \\
\text { peptidyl-serine } \\
\text { phosphorylation }\end{array}$ & $\begin{array}{l}1.00 \mathrm{E}- \\
02\end{array}$ & & \\
\hline & & & $\begin{array}{l}\text { positive regulation of } \\
\text { TOR signaling }\end{array}$ & $\begin{array}{l}1.00 \mathrm{E} \\
-02\end{array}$ & & \\
\hline & & & $\begin{array}{l}\text { negative regulation of I- } \\
\text { kappaB kinase/NF- } \\
\text { kappaB signaling }\end{array}$ & $\begin{array}{l}7.70 \mathrm{E} \\
-05\end{array}$ & & \\
\hline
\end{tabular}




\begin{tabular}{|c|c|c|c|c|c|c|}
\hline & \multicolumn{2}{|l|}{$\underline{1 \text { Hour }}$} & \multicolumn{2}{|l|}{$\underline{3 \text { Hour }}$} & \multicolumn{2}{|l|}{$\underline{6 \text { Hour }}$} \\
\hline & Process & $\begin{array}{c}\mathbf{P}- \\
\text { value }\end{array}$ & Process & $\begin{array}{c}\text { P- } \\
\text { value }\end{array}$ & Process & $\begin{array}{c}\text { P- } \\
\text { value }\end{array}$ \\
\hline \multirow{7}{*}{ 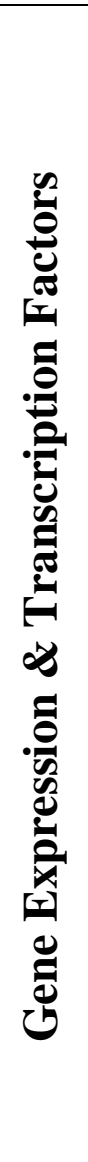 } & $\begin{array}{l}\text { negative regulation of } \\
\text { transcription from RNA } \\
\text { polymerase II promoter }\end{array}$ & $\begin{array}{l}1.70 \mathrm{E}- \\
05\end{array}$ & $\begin{array}{l}\text { positive regulation of } \\
\text { NF-kappaB } \\
\text { transcription factor } \\
\text { activity }\end{array}$ & $\begin{array}{l}1.70 \mathrm{E}- \\
04\end{array}$ & $\begin{array}{l}\text { negative regulation of } \\
\text { gene expression }\end{array}$ & $\begin{array}{l}1.10 \mathrm{E}- \\
02\end{array}$ \\
\hline & $\begin{array}{l}\text { positive regulation of } \\
\text { transcription from RNA } \\
\text { polymerase II promoter }\end{array}$ & $\begin{array}{c}2.50 \mathrm{E}- \\
05\end{array}$ & $\begin{array}{l}\text { positive regulation of I- } \\
\text { kappaB kinase/NF- } \\
\text { kappaB signaling }\end{array}$ & $\begin{array}{l}1.10 \mathrm{E}- \\
03\end{array}$ & $\begin{array}{l}\text { positive regulation of } \\
\text { NF-kappaB transcription } \\
\text { factor activity }\end{array}$ & $\begin{array}{c}8.40 \mathrm{E}- \\
04\end{array}$ \\
\hline & $\begin{array}{l}\text { positive regulation of } \\
\text { tyrosine phosphorylation } \\
\text { of Stat } 3 \text { protein }\end{array}$ & $\begin{array}{l}4.20 \mathrm{E}- \\
05\end{array}$ & $\begin{array}{l}\text { positive regulation of } \\
\text { protein kinase B } \\
\text { signaling }\end{array}$ & $\begin{array}{l}2.30 \mathrm{E}- \\
03\end{array}$ & $\begin{array}{l}\text { positive regulation of I- } \\
\text { kappaB kinase/NF- } \\
\text { kappaB signaling }\end{array}$ & $\begin{array}{l}\text { 7.10E- } \\
03\end{array}$ \\
\hline & & & $\begin{array}{l}\text { positive regulation of } \\
\text { NF-kappaB import into } \\
\text { nucleus }\end{array}$ & $\begin{array}{l}2.70 \mathrm{E}- \\
03\end{array}$ & $\begin{array}{l}\text { negative regulation of I- } \\
\text { kappaB kinase/NF- } \\
\text { kappaB signaling }\end{array}$ & $\begin{array}{l}8.30 \mathrm{E}- \\
03\end{array}$ \\
\hline & & & $\begin{array}{l}\text { regulation of I-kappaB } \\
\text { kinase/NF-kappaB } \\
\text { signaling }\end{array}$ & $\begin{array}{l}\text { 3.00E- } \\
03\end{array}$ & $\begin{array}{l}\text { regulation of I-kappaB } \\
\text { kinase/NF-kappaB } \\
\text { signaling }\end{array}$ & $\begin{array}{l}1.70 \mathrm{E}- \\
02\end{array}$ \\
\hline & & & $\begin{array}{l}\text { positive regulation of } \\
\text { transcription, DNA- } \\
\text { templated }\end{array}$ & $\begin{array}{l}1.00 \mathrm{E}- \\
02\end{array}$ & $\begin{array}{l}\text { negative regulation of } \\
\text { NF-kappaB transcription } \\
\text { factor activity }\end{array}$ & $\begin{array}{l}1.80 \mathrm{E}- \\
02\end{array}$ \\
\hline & & & $\begin{array}{l}\text { negative regulation of } \\
\text { transcription from RNA } \\
\text { polymerase II promoter }\end{array}$ & $\begin{array}{l}1.00 \mathrm{E}- \\
02\end{array}$ & & \\
\hline
\end{tabular}




\begin{tabular}{|c|c|c|c|c|c|c|}
\hline & \multicolumn{2}{|l|}{1 Hour } & \multicolumn{2}{|l|}{3 Hour } & \multicolumn{2}{|l|}{6 Hour } \\
\hline & Process & $\begin{array}{c}\text { P- } \\
\text { value }\end{array}$ & Process & $\begin{array}{c}\text { P- } \\
\text { value }\end{array}$ & Process & $\begin{array}{c}\text { P- } \\
\text { value }\end{array}$ \\
\hline \multirow{8}{*}{ 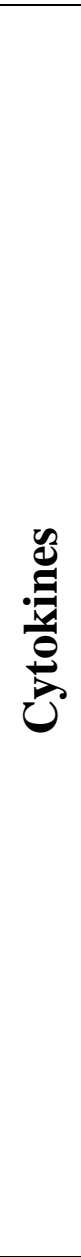 } & $\begin{array}{l}\text { chemokine-mediated } \\
\text { signaling pathway }\end{array}$ & $\begin{array}{l}4.60 \mathrm{E}- \\
08\end{array}$ & $\begin{array}{l}\text { cellular response to } \\
\text { tumor necrosis factor }\end{array}$ & $\begin{array}{l}8.30 \mathrm{E}- \\
06\end{array}$ & $\begin{array}{l}\text { interferon-gamma- } \\
\text { mediated signaling } \\
\text { pathway }\end{array}$ & $\begin{array}{l}2.90 \mathrm{E}- \\
06\end{array}$ \\
\hline & $\begin{array}{l}\text { cellular response to } \\
\text { tumor necrosis factor }\end{array}$ & $\begin{array}{c}2.00 \mathrm{E}- \\
07\end{array}$ & $\begin{array}{l}\text { positive regulation of } \\
\text { tumor necrosis factor } \\
\text { production }\end{array}$ & $\begin{array}{l}1.60 \mathrm{E}- \\
05\end{array}$ & $\begin{array}{l}\text { positive regulation of } \\
\text { interleukin- } 1 \text { beta } \\
\text { secretion }\end{array}$ & $\begin{array}{l}3.90 \mathrm{E}- \\
05\end{array}$ \\
\hline & $\begin{array}{l}\text { cellular response to } \\
\text { interleukin-1 }\end{array}$ & $\begin{array}{l}6.60 \mathrm{E}- \\
07\end{array}$ & $\begin{array}{l}\text { chemokine-mediated } \\
\text { signaling pathway }\end{array}$ & $\begin{array}{l}3.00 \mathrm{E}- \\
05\end{array}$ & $\begin{array}{l}\text { negative regulation of } \\
\text { type I interferon } \\
\text { production }\end{array}$ & $\begin{array}{l}8.00 \mathrm{E}- \\
04\end{array}$ \\
\hline & $\begin{array}{l}\text { cellular response to } \\
\text { interferon-gamma }\end{array}$ & $\begin{array}{l}2.60 \mathrm{E}- \\
05\end{array}$ & $\begin{array}{l}\text { positive regulation of } \\
\text { interleukin-1 beta } \\
\text { secretion }\end{array}$ & $\begin{array}{l}8.30 \mathrm{E}- \\
05\end{array}$ & $\begin{array}{l}\text { tumor necrosis factor- } \\
\text { mediated signaling } \\
\text { pathway }\end{array}$ & $\begin{array}{l}3.40 \mathrm{E}- \\
03\end{array}$ \\
\hline & $\begin{array}{l}\text { tumor necrosis factor- } \\
\text { mediated signaling } \\
\text { pathway }\end{array}$ & $\begin{array}{l}7.50 \mathrm{E}- \\
03\end{array}$ & $\begin{array}{l}\text { cellular response to } \\
\text { interleukin-1 }\end{array}$ & $\begin{array}{l}4.10 \mathrm{E}- \\
04\end{array}$ & $\begin{array}{l}\text { positive regulation of } \\
\text { interferon-beta } \\
\text { production }\end{array}$ & $\begin{array}{l}\text { 6.00E- } \\
03\end{array}$ \\
\hline & & & $\begin{array}{l}\text { tumor necrosis factor- } \\
\text { mediated signaling } \\
\text { pathway }\end{array}$ & $\begin{array}{l}4.10 \mathrm{E}- \\
03\end{array}$ & $\begin{array}{l}\text { cellular response to } \\
\text { tumor necrosis factor }\end{array}$ & $\begin{array}{l}6.40 \mathrm{E}- \\
03\end{array}$ \\
\hline & & & $\begin{array}{l}\text { regulation of tumor } \\
\text { necrosis factor-mediated } \\
\text { signaling pathway }\end{array}$ & $\begin{array}{l}4.30 \mathrm{E}- \\
03\end{array}$ & $\begin{array}{l}\text { cellular response to } \\
\text { cytokine stimulus }\end{array}$ & $\begin{array}{l}6.50 \mathrm{E}- \\
03\end{array}$ \\
\hline & & & $\begin{array}{l}\text { negative regulation of } \\
\text { transforming growth } \\
\text { factor beta receptor } \\
\text { signaling pathway }\end{array}$ & $\begin{array}{l}5.10 \mathrm{E}- \\
03\end{array}$ & $\begin{array}{l}\text { positive regulation of } \\
\text { interleukin-1 beta } \\
\text { production }\end{array}$ & $\begin{array}{l}6.50 \mathrm{E}- \\
03\end{array}$ \\
\hline
\end{tabular}




\begin{tabular}{|c|c|c|c|c|c|c|}
\hline & \multicolumn{2}{|c|}{$\underline{1 \text { Hour }}$} & \multicolumn{2}{|l|}{$\underline{3 \text { Hour }}$} & \multicolumn{2}{|l|}{6 Hour } \\
\hline & Process & $\begin{array}{c}\text { P- } \\
\text { value }\end{array}$ & Process & $\begin{array}{c}\text { P- } \\
\text { value }\end{array}$ & Process & $\begin{array}{c}\text { P- } \\
\text { value }\end{array}$ \\
\hline \multirow{3}{*}{ 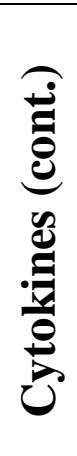 } & & & $\begin{array}{l}\text { positive regulation of } \\
\text { chemokine production }\end{array}$ & $\begin{array}{l}5.40 \mathrm{E}- \\
03\end{array}$ & $\begin{array}{l}\text { negative regulation of } \\
\text { transforming growth } \\
\text { factor beta receptor } \\
\text { signaling pathway }\end{array}$ & $\begin{array}{l}7.20 \mathrm{E}- \\
03\end{array}$ \\
\hline & & & $\begin{array}{l}\text { positive regulation of } \\
\text { interleukin-8 production }\end{array}$ & $\begin{array}{l}8.60 \mathrm{E}- \\
03\end{array}$ & $\begin{array}{l}\text { chemokine-mediated } \\
\text { signaling pathway }\end{array}$ & $\begin{array}{l}7.70 \mathrm{E}- \\
03\end{array}$ \\
\hline & & & & & $\begin{array}{l}\text { type I interferon signaling } \\
\text { pathway }\end{array}$ & $\begin{array}{l}7.20 \mathrm{E}- \\
03\end{array}$ \\
\hline \multirow{7}{*}{ 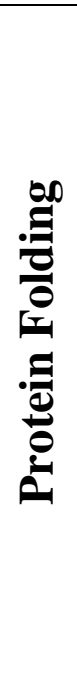 } & & & & & $\begin{array}{l}\text { response to unfolded } \\
\text { protein }\end{array}$ & $\begin{array}{l}1.40 \mathrm{E}- \\
08\end{array}$ \\
\hline & & & & & protein folding & $\begin{array}{l}1.50 \mathrm{E}- \\
05\end{array}$ \\
\hline & & & & & protein refolding & $\begin{array}{l}5.70 \mathrm{E}- \\
05\end{array}$ \\
\hline & & & & & protein ubiquitination & $\begin{array}{l}1.60 \mathrm{E}- \\
02\end{array}$ \\
\hline & & & & & protein maturation & $\begin{array}{l}1.70 \mathrm{E}- \\
02\end{array}$ \\
\hline & & & & & $\begin{array}{l}\text { chaperone-mediated } \\
\text { protein complex assembly }\end{array}$ & $\begin{array}{l}8.80 \mathrm{E}- \\
03\end{array}$ \\
\hline & & & & & $\begin{array}{l}\text { positive regulation of } \\
\text { protein phosphorylation }\end{array}$ & $\begin{array}{l}9.10 \mathrm{E}- \\
04\end{array}$ \\
\hline
\end{tabular}




\begin{tabular}{|c|c|c|c|c|c|c|}
\hline & \multicolumn{2}{|l|}{1 Hour } & \multicolumn{2}{|l|}{3 Hour } & \multicolumn{2}{|l|}{6 Hour } \\
\hline & Process & $\begin{array}{c}P \text { - } \\
\text { value }\end{array}$ & Process & $\begin{array}{c}\mathrm{P}- \\
\text { value }\end{array}$ & Process & $\begin{array}{c}\mathbf{P}- \\
\text { value }\end{array}$ \\
\hline \multirow{11}{*}{ 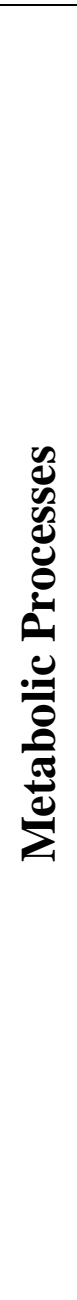 } & $\begin{array}{l}\text { positive regulation of } \\
\text { nitric oxide biosynthetic } \\
\text { process }\end{array}$ & $\begin{array}{l}9.80 \mathrm{E}- \\
03\end{array}$ & $\begin{array}{l}\text { glycosphingolipid } \\
\text { metabolic process }\end{array}$ & $\begin{array}{l}2.40 \mathrm{E}- \\
04\end{array}$ & $\begin{array}{l}\text { glycosphingolipid } \\
\text { metabolic process }\end{array}$ & $\begin{array}{l}2.30 \mathrm{E}- \\
03\end{array}$ \\
\hline & response to amino acid & $\begin{array}{l}3.90 \mathrm{E}- \\
03\end{array}$ & $\begin{array}{l}\text { cellular response to } \\
\text { starvation }\end{array}$ & $\begin{array}{l}1.50 \mathrm{E}- \\
03\end{array}$ & $\begin{array}{l}\text { positive regulation of } \\
\text { tumor necrosis factor } \\
\text { production }\end{array}$ & $\begin{array}{l}3.30 \mathrm{E}- \\
03\end{array}$ \\
\hline & $\begin{array}{l}\text { cellular response to } \\
\text { glucocorticoid stimulus }\end{array}$ & $\begin{array}{l}1.10 \mathrm{E}- \\
03\end{array}$ & $\begin{array}{l}\text { positive regulation of } \\
\text { nitric oxide biosynthetic } \\
\text { process }\end{array}$ & $\begin{array}{l}2.80 \mathrm{E}- \\
03\end{array}$ & GTP metabolic process & $\begin{array}{l}3.60 \mathrm{E}- \\
03\end{array}$ \\
\hline & $\begin{array}{l}\text { cellular response to } \\
\text { corticotropin-releasing } \\
\text { hormone stimulus }\end{array}$ & $\begin{array}{l}1.50 \mathrm{E}- \\
03\end{array}$ & $\begin{array}{l}\text { cellular response to fatty } \\
\text { acid }\end{array}$ & $\begin{array}{l}4.50 \mathrm{E}- \\
03\end{array}$ & $\begin{array}{l}\text { proteasomal ubiquitin- } \\
\text { independent protein } \\
\text { catabolic process }\end{array}$ & $\begin{array}{l}4.60 \mathrm{E}- \\
03\end{array}$ \\
\hline & & & $\begin{array}{l}\text { UDP-N- } \\
\text { acetylglucosamine } \\
\text { biosynthetic process }\end{array}$ & $\begin{array}{l}5.60 \mathrm{E}- \\
03\end{array}$ & $\begin{array}{l}\text { glycogen biosynthetic } \\
\text { process }\end{array}$ & $\begin{array}{l}4.70 \mathrm{E}- \\
03\end{array}$ \\
\hline & & & $\begin{array}{l}\mathrm{N} \text {-acetylneuraminate } \\
\text { catabolic process }\end{array}$ & $\begin{array}{l}6.10 \mathrm{E}- \\
03\end{array}$ & $\begin{array}{l}\text { carbohydrate metabolic } \\
\text { process }\end{array}$ & $\begin{array}{l}1.10 \mathrm{E}- \\
02\end{array}$ \\
\hline & & & $\begin{array}{l}\text { positive regulation of } \\
\text { cholesterol homeostasis }\end{array}$ & $\begin{array}{l}6.10 \mathrm{E}- \\
03\end{array}$ & $\begin{array}{l}\text { phospholipid metabolic } \\
\text { process }\end{array}$ & $\begin{array}{l}1.10 \mathrm{E}- \\
02\end{array}$ \\
\hline & & & ATP metabolic process & $\begin{array}{l}6.30 \mathrm{E}- \\
03\end{array}$ & $\begin{array}{l}\text { regulation of } \\
\text { gluconeogenesis }\end{array}$ & $\begin{array}{l}1.10 \mathrm{E}- \\
02\end{array}$ \\
\hline & & & $\begin{array}{l}\text { ATP hydrolysis coupled } \\
\text { proton transport }\end{array}$ & $\begin{array}{l}6.30 \mathrm{E}- \\
03\end{array}$ & $\begin{array}{l}\text { inositol phosphate } \\
\text { metabolic process }\end{array}$ & $\begin{array}{l}9.70 \mathrm{E}- \\
03\end{array}$ \\
\hline & & & proton transport & $\begin{array}{l}2.60 \mathrm{E}- \\
05\end{array}$ & $\begin{array}{l}\text { ceramide biosynthetic } \\
\text { process }\end{array}$ & $\begin{array}{l}1.40 \mathrm{E}- \\
02\end{array}$ \\
\hline & & & & & $\mathrm{N}$-glycan processing & $\begin{array}{l}1.60 \mathrm{E}- \\
02\end{array}$ \\
\hline
\end{tabular}




\begin{tabular}{|c|c|c|c|c|c|c|}
\hline & \multicolumn{2}{|l|}{1 Hour } & \multicolumn{2}{|l|}{3 Hour } & \multicolumn{2}{|l|}{6 Hour } \\
\hline & Process & $\begin{array}{l}\text { P- } \\
\text { value }\end{array}$ & Process & $\begin{array}{l}\text { P- } \\
\text { value }\end{array}$ & Process & $\begin{array}{l}\text { P- } \\
\text { value }\end{array}$ \\
\hline \multirow{2}{*}{ 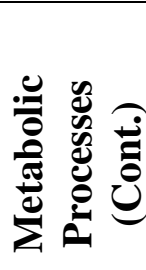 } & & & & & $\begin{array}{l}\text { N-acetylneuraminate } \\
\text { catabolic process }\end{array}$ & $\begin{array}{l}1.70 \mathrm{E}- \\
03\end{array}$ \\
\hline & & & & & $\begin{array}{l}\text { positive regulation of } \\
\text { MHC class I biosynthetic } \\
\text { process }\end{array}$ & $\begin{array}{l}1.90 \mathrm{E}- \\
02\end{array}$ \\
\hline \multirow{5}{*}{$\frac{\frac{n}{\pi}}{\frac{0}{0}} \frac{0}{\frac{0}{2}}$} & $\begin{array}{l}\text { negative regulation of } \\
\text { apoptotic process }\end{array}$ & $\begin{array}{l}2.00 \mathrm{E}- \\
05\end{array}$ & $\begin{array}{l}\text { negative regulation of } \\
\text { apoptotic process }\end{array}$ & $\begin{array}{l}2.10 \mathrm{E}- \\
05\end{array}$ & apoptotic process & $\begin{array}{l}3.70 \mathrm{E}- \\
07\end{array}$ \\
\hline & apoptotic process & $\begin{array}{c}7.90 \mathrm{E}- \\
04\end{array}$ & apoptotic process & $\begin{array}{l}1.60 \mathrm{E}- \\
04\end{array}$ & $\begin{array}{l}\text { apoptotic mitochondrial } \\
\text { changes }\end{array}$ & $\begin{array}{l}2.60 \mathrm{E}- \\
03\end{array}$ \\
\hline & $\begin{array}{l}\text { activation of cysteine- } \\
\text { type endopeptidase } \\
\text { activity involved in } \\
\text { apoptotic process }\end{array}$ & $\begin{array}{l}1.70 \mathrm{E}- \\
03\end{array}$ & $\begin{array}{l}\text { positive regulation of } \\
\text { apoptotic process }\end{array}$ & $\begin{array}{l}1.90 \mathrm{E}- \\
04\end{array}$ & $\begin{array}{l}\text { extrinsic apoptotic } \\
\text { signaling pathway }\end{array}$ & $\begin{array}{l}1.20 \mathrm{E}- \\
02\end{array}$ \\
\hline & & & necroptotic process & $\begin{array}{l}1.10 \mathrm{E}- \\
03\end{array}$ & $\begin{array}{l}\text { activation of cysteine-type } \\
\text { endopeptidase activity } \\
\text { involved in apoptotic } \\
\text { process }\end{array}$ & $\begin{array}{l}4.00 \mathrm{E}- \\
04\end{array}$ \\
\hline & & & $\begin{array}{l}\text { negative regulation of } \\
\text { extrinsic apoptotic } \\
\text { signaling pathway via } \\
\text { death domain receptors }\end{array}$ & $\begin{array}{l}1.80 \mathrm{E}- \\
03\end{array}$ & $\begin{array}{l}\text { activation of cysteine-type } \\
\text { endopeptidase activity } \\
\text { involved in apoptotic } \\
\text { signaling pathway }\end{array}$ & $\begin{array}{l}1.30 \mathrm{E}- \\
03\end{array}$ \\
\hline
\end{tabular}




\begin{tabular}{|c|c|c|c|c|c|c|}
\hline & \multicolumn{2}{|l|}{1 Hour } & \multicolumn{2}{|l|}{3 Hour } & \multicolumn{2}{|l|}{6 Hour } \\
\hline & Process & $\begin{array}{l}\text { P- } \\
\text { value }\end{array}$ & Process & $\begin{array}{l}\text { P- } \\
\text { value }\end{array}$ & Process & $\begin{array}{l}\text { P- } \\
\text { value }\end{array}$ \\
\hline \multirow{4}{*}{ 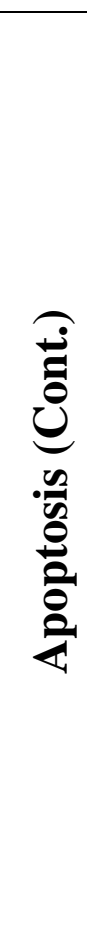 } & & & $\begin{array}{l}\text { negative regulation of } \\
\text { cysteine-type } \\
\text { endopeptidase activity } \\
\text { involved in apoptotic } \\
\text { process }\end{array}$ & $\begin{array}{l}3.20 \mathrm{E}- \\
03\end{array}$ & $\begin{array}{l}\text { negative regulation of } \\
\text { extrinsic apoptotic } \\
\text { signaling pathway via } \\
\text { death domain receptors }\end{array}$ & $\begin{array}{l}6.60 \mathrm{E}- \\
03\end{array}$ \\
\hline & & & $\begin{array}{l}\text { intrinsic apoptotic } \\
\text { signaling pathway in } \\
\text { response to DNA } \\
\text { damage }\end{array}$ & $\begin{array}{l}5.30 \mathrm{E}- \\
03\end{array}$ & $\begin{array}{l}\text { regulation of extrinsic } \\
\text { apoptotic signaling } \\
\text { pathway via death domain } \\
\text { receptors }\end{array}$ & $\begin{array}{l}6.70 \mathrm{E}- \\
03\end{array}$ \\
\hline & & & $\begin{array}{l}\text { regulation of extrinsic } \\
\text { apoptotic signaling } \\
\text { pathway via death } \\
\text { domain receptors }\end{array}$ & $\begin{array}{l}5.40 \mathrm{E}- \\
03\end{array}$ & $\begin{array}{l}\text { negative regulation of } \\
\text { apoptotic process }\end{array}$ & $\begin{array}{l}1.10 \mathrm{E}- \\
02\end{array}$ \\
\hline & & & $\begin{array}{l}\text { activation of cysteine- } \\
\text { type endopeptidase } \\
\text { activity involved in } \\
\text { apoptotic process }\end{array}$ & $\begin{array}{l}5.60 \mathrm{E}- \\
03\end{array}$ & $\begin{array}{l}\text { positive regulation of } \\
\text { apoptotic process }\end{array}$ & $\begin{array}{l}1.10 \mathrm{E}- \\
03\end{array}$ \\
\hline \multirow{3}{*}{ 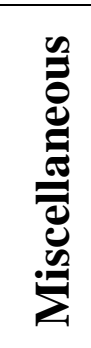 } & $\begin{array}{l}\text { positive regulation of } \\
\text { osteoclast differentiation }\end{array}$ & $\begin{array}{l}3.70 \mathrm{E}- \\
05\end{array}$ & $\begin{array}{l}\text { positive regulation of } \\
\text { osteoclast differentiation }\end{array}$ & $\begin{array}{l}1.60 \mathrm{E}- \\
03\end{array}$ & $\begin{array}{l}\text { endoplasmic reticulum } \\
\text { calcium ion homeostasis }\end{array}$ & $\begin{array}{l}3.30 \mathrm{E}- \\
03\end{array}$ \\
\hline & \begin{tabular}{|l|} 
cell adhesion \\
\end{tabular} & $\begin{array}{l}8.10 \mathrm{E}- \\
03\end{array}$ & $\begin{array}{l}\text { positive regulation of } \\
\text { calcium ion transport }\end{array}$ & $\begin{array}{l}8.60 \mathrm{E}- \\
03\end{array}$ & cell-cell adhesion & $\begin{array}{l}3.50 \mathrm{E}- \\
03\end{array}$ \\
\hline & & & & & secretion by cell & $\begin{array}{l}1.60 \mathrm{E}- \\
02\end{array}$ \\
\hline
\end{tabular}




\begin{tabular}{|c|c|c|c|c|c|c|}
\hline & \multicolumn{2}{|c|}{1 Hour } & \multicolumn{2}{|c|}{3 Hour } & \multicolumn{2}{|l|}{6 Hour } \\
\hline & Process & $\begin{array}{l}\text { P- } \\
\text { value }\end{array}$ & Process & $\begin{array}{l}\text { P- } \\
\text { value }\end{array}$ & Process & $\begin{array}{l}\text { P- } \\
\text { value }\end{array}$ \\
\hline \multirow{5}{*}{ 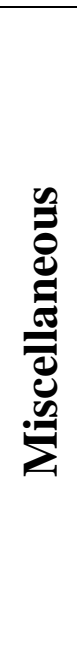 } & & & & & proton transport & $\begin{array}{l}4.80 \mathrm{E}- \\
03\end{array}$ \\
\hline & & & & & response to iron ion & $\begin{array}{l}9.10 \mathrm{E}- \\
03\end{array}$ \\
\hline & & & & & $\begin{array}{l}\text { negative regulation of } \\
\text { protein serine/threonine } \\
\text { kinase activity }\end{array}$ & $\begin{array}{l}9.10 \mathrm{E}- \\
03\end{array}$ \\
\hline & & & & & $\begin{array}{l}\text { negative regulation of } \\
\text { cysteine-type } \\
\text { endopeptidase activity } \\
\text { involved in apoptotic } \\
\text { process }\end{array}$ & $\begin{array}{l}1.40 \mathrm{E}- \\
02\end{array}$ \\
\hline & & & & & $\begin{array}{l}\text { positive regulation of } \\
\text { calcium ion transport }\end{array}$ & $\begin{array}{l}4.70 \mathrm{E}- \\
03\end{array}$ \\
\hline
\end{tabular}


Table 2.3: Genes involved in the Biological Process of Inflammation \& Neutrophil activation by MetaCore

\begin{tabular}{|c|c|c|c|c|c|c|c|}
\hline \multirow[b]{2}{*}{ Input IDs } & \multirow[b]{2}{*}{ Network Object Name } & \multicolumn{2}{|c|}{1 Hour } & \multicolumn{2}{|c|}{3 Hour } & \multicolumn{2}{|c|}{6 Hour } \\
\hline & & $\begin{array}{c}\text { Log2Fold } \\
\text { Change }\end{array}$ & p-value & $\begin{array}{c}\text { Log2Fold } \\
\text { Change }\end{array}$ & p-value & $\begin{array}{c}\text { Log2Fold } \\
\text { Change }\end{array}$ & p-value \\
\hline ENSG00000142208 & AKT(PKB) & -0.268394 & 0.56125 & -1.35053 & 0.0029 & -2.14563 & 0.00005 \\
\hline ENSG00000012779 & ALOX5 & -0.235755 & 0.46905 & -0.43537 & 0.17815 & -1.30551 & 0.00025 \\
\hline ENSG00000115966 & AP-1 & 0.336876 & 0.41865 & 0.492399 & 0.23355 & 0.730992 & 0.0755 \\
\hline ENSG00000115966 & ATF-2/c-Jun & 0.336876 & 0.41865 & 0.492399 & 0.23355 & 0.730992 & 0.0755 \\
\hline ENSG00000143632 & Actin & 0.636624 & 1 & -1.3805 & 1 & -0.975943 & 1 \\
\hline ENSG00000164742 & Adenylate cyclase & 1.20705 & 1 & 0.372034 & 1 & 0.698685 & 1 \\
\hline ENSG00000164742 & Adenylate cyclase type I & 1.20705 & 1 & 0.372034 & 1 & 0.698685 & 1 \\
\hline ENSG00000078295 & $\begin{array}{l}\text { Adenylate cyclase type } \\
\text { II }\end{array}$ & 0.948255 & 1 & 0.638634 & 1 & 0.867516 & 1 \\
\hline ENSG00000138031 & $\begin{array}{l}\text { Adenylate cyclase type } \\
\text { III }\end{array}$ & 0.214679 & 0.767 & 3.72654 & 0.00005 & 4.60815 & 0.00005 \\
\hline ENSG00000129467 & $\begin{array}{l}\text { Adenylate cyclase type } \\
\text { IV }\end{array}$ & -0.906962 & 0.0755 & -0.65237 & 0.1834 & -1.09539 & 0.02525 \\
\hline ENSG00000162104 & $\begin{array}{l}\text { Adenylate cyclase type } \\
\text { IX }\end{array}$ & 0.704655 & 1 & 2.14178 & 0.0006 & 2.31121 & 0.0002 \\
\hline ENSG00000173175 & $\begin{array}{l}\text { Adenylate cyclase type } \\
\text { V }\end{array}$ & -4.53876 & 1 & 0.0994383 & 1 & -2.46958 & 1 \\
\hline ENSG00000174233 & $\begin{array}{l}\text { Adenylate cyclase type } \\
\text { VI }\end{array}$ & 0.173814 & 1 & 0.654431 & 1 & 0.28185 & 1 \\
\hline ENSG00000121281 & $\begin{array}{l}\text { Adenylate cyclase type } \\
\text { VII }\end{array}$ & -0.605382 & 0.2165 & -0.429987 & 0.3534 & -0.103959 & 0.83925 \\
\hline ENSG00000155897 & $\begin{array}{l}\text { Adenylate cyclase type } \\
\text { VIII }\end{array}$ & -15.5693 & 1 & 0.222025 & 1 & 2.16771 & 1 \\
\hline ENSG00000135046 & Annexin I & 0.240346 & 0.4812 & -0.660925 & 0.0554 & -1.59915 & 0.00015 \\
\hline ENSG00000120868 & Apaf-1 & -0.147656 & 0.73435 & -0.852756 & 0.06645 & -0.543003 & 0.23705 \\
\hline ENSG00000138071 & Arp2/3 & -0.085798 & 0.8165 & -0.290621 & 0.39975 & -0.611465 & 0.1097 \\
\hline ENSG00000015475 & Bid & 0.106129 & 0.8675 & 1.5263 & 0.0072 & 0.85196 & 0.169 \\
\hline
\end{tabular}




\begin{tabular}{|c|c|c|c|c|c|c|c|}
\hline \multirow[b]{2}{*}{ Input IDs } & \multirow[b]{2}{*}{ Network Object Name } & \multicolumn{2}{|c|}{1 hour } & \multicolumn{2}{|c|}{3 hour } & \multicolumn{2}{|c|}{6 hour } \\
\hline & & $\begin{array}{l}\text { Log2Fold } \\
\text { Change }\end{array}$ & p-value & $\begin{array}{l}\text { Log2Fold } \\
\text { Change }\end{array}$ & p-value & $\begin{array}{l}\text { Log2Fold } \\
\text { Change }\end{array}$ & p-value \\
\hline ENSG00000010671 & Btk & -0.151721 & 0.70195 & -1.02335 & 0.0073 & 0.172335 & 0.69585 \\
\hline ENSG00000106804 & C5a & -0.670077 & 0.3237 & 0.572423 & 0.49415 & 0.942053 & 0.25 \\
\hline ENSG00000197405 & $\mathrm{C} 5 \mathrm{aR}$ & 0.291758 & 0.38 & -0.129738 & 0.7045 & -1.24779 & 0.0008 \\
\hline ENSG00000108691 & CCL2 & 2.84034 & 0.00005 & 1.91775 & 0.0006 & 0.95627 & 0.06905 \\
\hline ENSG00000135404 & CD63 & 0.341514 & 0.49925 & 2.20922 & 0.00005 & 2.74768 & 0.00005 \\
\hline ENSG00000006210 & CX3CL1 & 0.412438 & 1 & 0.807216 & 1 & 0.31547 & 1 \\
\hline ENSG00000168329 & CX3CR1 & -0.154489 & 0.76035 & -0.579668 & 0.35505 & -0.20214 & 0.6979 \\
\hline ENSG00000164305 & Caspase-3 & 0.0872686 & 0.80205 & 0.340595 & 0.3449 & 0.796916 & 0.0377 \\
\hline ENSG00000064012 & Caspase- 8 & -0.332073 & 0.4657 & -1.05957 & 0.0224 & -1.78981 & 0.00025 \\
\hline ENSG00000172757 & Cofilin & -0.019265 & 0.96085 & -0.29201 & 0.4632 & -1.2949 & 0.00155 \\
\hline ENSG00000051523 & Cytochrome b-558 & -0.234277 & 0.64875 & 0.438144 & 0.3666 & 0.201541 & 0.6868 \\
\hline ENSG00000172115 & Cytochrome c & 0.401099 & 0.43565 & -0.174956 & 0.7352 & -0.420116 & 0.43985 \\
\hline ENSG00000168970 & $\begin{array}{l}\text { Cytosolic phospholipase } \\
\text { A2 beta }\end{array}$ & -0.649148 & 0.4939 & -0.859994 & 0.3485 & -1.22401 & 0.20455 \\
\hline ENSG00000007908 & E-selectin & 0 & 1 & 15.6737 & 1 & 14.7034 & 1 \\
\hline ENSG00000163735 & ENA-78 & 2.78973 & 1 & 4.99307 & 0.0094 & 4.94474 & 0.0082 \\
\hline ENSG00000102882 & ERK1 (MAPK3) & -0.31347 & 0.50515 & -1.46191 & 0.0036 & -1.83436 & 0.00085 \\
\hline ENSG00000100030 & ERK1/2 & 0.111859 & 0.86515 & -1.22384 & 0.04215 & -2.03661 & 0.00205 \\
\hline ENSG00000100030 & ERK2 (MAPK1) & 0.111859 & 0.86515 & -1.22384 & 0.04215 & -2.03661 & 0.00205 \\
\hline ENSG00000168040 & FADD & -1.82699 & 0.02235 & -1.83039 & 0.0195 & -2.24993 & 0.00495 \\
\hline ENSG00000171051 & FPR & 0.309293 & 0.4953 & 0.433394 & 0.33985 & -0.117564 & 0.80285 \\
\hline ENSG00000171049 & FPRL1 & 0.300916 & 0.7945 & 0.258945 & 0.80795 & -0.704088 & 0.52475 \\
\hline ENSG00000060558 & G-protein alpha-15 & 0.108801 & 0.77065 & 1.07998 & 0.00265 & 1.11406 & 0.0034 \\
\hline ENSG00000127955 & G-protein alpha-i family & 0.571501 & 1 & -12.529 & 1 & -2.51315 & 1 \\
\hline ENSG00000114353 & G-protein alpha-i2 & -0.334653 & 0.3361 & -0.98326 & 0.004 & -2.03799 & 0.00005 \\
\hline
\end{tabular}




\begin{tabular}{|c|c|c|c|c|c|c|c|}
\hline \multirow[b]{2}{*}{ Input IDs } & \multirow[b]{2}{*}{ Network Object Name } & \multicolumn{2}{|c|}{1 hour } & \multicolumn{2}{|c|}{3 hour } & \multicolumn{2}{|c|}{6 hour } \\
\hline & & $\begin{array}{l}\text { Log2Fold } \\
\text { Change }\end{array}$ & p-value & $\begin{array}{l}\text { Log2Fold } \\
\text { Change }\end{array}$ & p-value & $\begin{array}{l}\text { Log2Fold } \\
\text { Change }\end{array}$ & p-value \\
\hline ENSG00000088256 & G-protein alpha-q/11 & -0.383532 & 0.6306 & -0.040169 & 0.9544 & 0.202808 & 0.78065 \\
\hline ENSG00000078369 & G-protein beta/gamma & -0.003427 & 0.99495 & 0.695778 & 0.17615 & 0.279574 & 0.6152 \\
\hline ENSG00000177885 & GRB2 & 0.162711 & 0.7157 & 0.776796 & 0.06805 & 0.653148 & 0.16675 \\
\hline ENSG00000163739 & GRO-1 & 3.37405 & 0.00005 & 1.8271 & 0.00005 & 1.77034 & 0.00005 \\
\hline ENSG00000081041 & GRO-2 & 6.33583 & 0.00005 & 6.15229 & 0.00005 & 6.00946 & 0.00005 \\
\hline ENSG00000163734 & GRO-3 & 4.37038 & 0.00005 & 3.77473 & 0.00005 & 4.35262 & 0.00005 \\
\hline ENSG00000144648 & $\begin{array}{l}\text { Galpha(i)-specific } \\
\text { peptide GPCRs }\end{array}$ & -1.48914 & 0.04105 & -1.18726 & 0.09355 & -1.35623 & 0.064 \\
\hline ENSG00000174775 & H-Ras & -0.079523 & 0.88715 & -0.100795 & 0.88615 & -2.22536 & 0.00045 \\
\hline ENSG00000100906 & $\mathrm{I}-\mathrm{kB}$ & 0 & 1 & 3.16244 & 0.00005 & 2.48684 & 0.00005 \\
\hline ENSG00000090339 & ICAM1 & 2.80958 & 0.00005 & 2.10994 & 0.00005 & 1.37142 & 0.00605 \\
\hline ENSG00000108622 & ICAM2 & -0.172743 & 0.70855 & 0.353612 & 0.4279 & -0.709392 & 0.13385 \\
\hline ENSG00000105371 & ICAM4 & 2.97046 & 0.0036 & 2.5412 & 0.00365 & 2.49967 & 0.00435 \\
\hline ENSG00000111537 & IFN-gamma & -0.411676 & 1 & -1.57507 & 1 & -1.43862 & 1 \\
\hline ENSG00000213341 & IKK (cat) & 0.0386051 & 0.9263 & -0.201073 & 0.64655 & -0.033242 & 0.93635 \\
\hline ENSG00000213341 & IKK-alpha & 0.0386051 & 0.9263 & -0.201073 & 0.64655 & -0.033242 & 0.93635 \\
\hline ENSG00000104365 & IKK-beta & -0.24989 & 0.576 & 1.24499 & 0.0115 & 1.03277 & 0.0572 \\
\hline ENSG00000136634 & IL-10 & -0.882243 & 0.1873 & -0.420915 & 0.48805 & 0.508715 & 0.52685 \\
\hline ENSG00000109471 & IL-2 & 0 & 1 & 15.6737 & 1 & 0 & 1 \\
\hline ENSG00000134460 & IL-2 receptor & 1.67419 & 1 & 4.1224 & 0.0016 & 3.33951 & 0.0022 \\
\hline ENSG00000100385 & IL-2R beta chain & 0.432867 & 1 & 0.504894 & 1 & 1.06171 & 0.29405 \\
\hline ENSG00000113525 & IL-5 & 0.242808 & 1 & 1.40525 & 1 & 1.79082 & 1 \\
\hline ENSG00000136244 & IL-6 & 2.99727 & 0.08685 & 6.22286 & 0.0654 & 5.70078 & 0.0652 \\
\hline ENSG00000163464 & IL8RA & -0.746133 & 0.023 & -2.18534 & 0.00005 & -1.99003 & 0.00005 \\
\hline ENSG00000180871 & IL8RB & -0.329611 & 0.40595 & -1.94377 & 0.00005 & -2.21343 & 0.00005 \\
\hline
\end{tabular}




\begin{tabular}{|c|c|c|c|c|c|c|c|}
\hline \multirow[b]{2}{*}{ Input IDs } & \multirow[b]{2}{*}{ Network Object Name } & \multicolumn{2}{|c|}{1 hour } & \multicolumn{2}{|c|}{3 hour } & \multicolumn{2}{|c|}{6 hour } \\
\hline & & $\begin{array}{l}\text { Log2Fold } \\
\text { Change }\end{array}$ & p-value & $\begin{array}{l}\text { Log2Fold } \\
\text { Change }\end{array}$ & p-value & $\begin{array}{l}\text { Log2Fold } \\
\text { Change }\end{array}$ & p-value \\
\hline ENSG00000150995 & IP3 receptor & 0.0169103 & 0.9694 & 1.96326 & 0.00005 & 2.39845 & 0.00005 \\
\hline ENSG00000169896 & ITGAM & -0.072381 & 0.8493 & -0.199032 & 0.59635 & -0.635927 & 0.10645 \\
\hline ENSG00000160255 & ITGB2 & -0.277596 & 0.3734 & -0.768094 & 0.01255 & -2.11987 & 0.00005 \\
\hline ENSG00000162434 & JAK1 & 0.134832 & 0.66445 & 0.0062887 & 0.9844 & 0.463703 & 0.14465 \\
\hline ENSG00000175592 & JunB/Fra-1 & 0.700587 & 0.10625 & -1.95565 & 0.00005 & -2.27314 & 0.00005 \\
\hline ENSG00000170345 & JunD/c-Fos & 0.540993 & 0.1754 & -1.63712 & 0.00005 & -0.447981 & 0.2341 \\
\hline ENSG00000106683 & LIMK1 & -0.488078 & 0.22955 & -1.0831 & 0.00895 & -2.07483 & 0.00005 \\
\hline ENSG00000182541 & LIMK2 & 0.505974 & 0.16555 & 0.975505 & 0.0039 & 0.210261 & 0.54895 \\
\hline ENSG00000111144 & LTA4H & -0.18693 & 0.6781 & -0.627719 & 0.1415 & -0.426102 & 0.3342 \\
\hline ENSG00000213903 & LTBR1 & -0.811739 & 0.26125 & -1.3433 & 0.08285 & -2.08177 & 0.0134 \\
\hline ENSG00000213906 & LTBR2 & -1.221 & 0.41905 & -2.23305 & 0.17685 & -3.13535 & 0.091 \\
\hline ENSG00000110514 & MADD & 0.0831299 & 0.8343 & -0.649991 & 0.14525 & -0.791325 & 0.04255 \\
\hline ENSG00000156575 & MBPH & 0.9458 & 1 & 1.26023 & 1 & 2.31847 & 1 \\
\hline ENSG00000169032 & MEK1(MAP2K1) & -0.016729 & 0.9712 & -0.019278 & 0.9619 & -0.485772 & 0.3074 \\
\hline ENSG00000169032 & MEK1/2 & -0.016729 & 0.9712 & -0.019278 & 0.9619 & -0.485772 & 0.3074 \\
\hline ENSG00000126934 & MEK2(MAP2K2) & 0.0310253 & 0.9711 & -0.818938 & 0.27245 & -0.478587 & 0.58065 \\
\hline ENSG00000095015 & MEKK1(MAP3K1) & 0.0913193 & 0.7935 & -0.773331 & 0.02555 & 0.30483 & 0.38005 \\
\hline ENSG00000109320 & NF-kB & 1.4494 & 0.00125 & 3.07581 & 0.00005 & 2.37882 & 0.00005 \\
\hline ENSG00000109320 & NF-kB p50/c-Rel & 1.4494 & 0.00125 & 3.07581 & 0.00005 & 2.37882 & 0.00005 \\
\hline ENSG00000109320 & NF-kB p50/p50 & 1.4494 & 0.00125 & 3.07581 & 0.00005 & 2.37882 & 0.00005 \\
\hline ENSG00000109320 & NF-kB p50/p65 & 1.4494 & 0.00125 & 3.07581 & 0.00005 & 2.37882 & 0.00005 \\
\hline ENSG00000077150 & NF-kB p52/RelB & 1.65511 & 0.00005 & 2.46764 & 0.00005 & 1.84765 & 0.00005 \\
\hline ENSG00000162924 & NF-kB p65/c-Rel & 1.09213 & 0.0241 & 1.97707 & 0.00005 & 1.74788 & 0.00025 \\
\hline ENSG00000173039 & NF-kB p65/p65 & 0.805521 & 0.075 & 0.644145 & 0.14395 & -0.831558 & 0.0612 \\
\hline ENSG00000109320 & NF-kB1 (p50) & 1.4494 & 0.00125 & 3.07581 & 0.00005 & 2.37882 & 0.00005 \\
\hline
\end{tabular}




\begin{tabular}{|c|c|c|c|c|c|c|c|}
\hline \multirow[b]{2}{*}{ Input IDs } & \multirow[b]{2}{*}{ Network Object Name } & \multicolumn{2}{|c|}{1 hour } & \multicolumn{2}{|c|}{3 hour } & \multicolumn{2}{|c|}{6 hour } \\
\hline & & $\begin{array}{l}\text { Log2Fold } \\
\text { Change }\end{array}$ & p-value & $\begin{array}{l}\text { Log2Fold } \\
\text { Change }\end{array}$ & p-value & $\begin{array}{l}\text { Log2Fold } \\
\text { Change }\end{array}$ & p-value \\
\hline ENSG00000077150 & NF-kB2 (p100) & 1.65511 & 0.00005 & 2.46764 & 0.00005 & 1.84765 & 0.00005 \\
\hline ENSG00000077150 & NF-kB2 (p52) & 1.65511 & 0.00005 & 2.46764 & 0.00005 & 1.84765 & 0.00005 \\
\hline ENSG00000100906 & NFKBIA & 0 & 1 & 3.16244 & 0.00005 & 2.48684 & 0.00005 \\
\hline ENSG00000146232 & NFKBIE & 2.0777 & 0.0001 & 2.61055 & 0.00005 & 2.19574 & 0.00005 \\
\hline ENSG00000006062 & NIK(MAP3K14) & -0.560321 & 0.4498 & -1.84519 & 0.0133 & -1.53898 & 0.0294 \\
\hline ENSG00000007952 & NOX1 & -1.19698 & 1 & -0.310513 & 1 & -0.99699 & 1 \\
\hline ENSG00000051523 & NOX1/p22-phox & -0.234277 & 0.64875 & 0.438144 & 0.3666 & 0.201541 & 0.6868 \\
\hline ENSG00000117592 & NSGPeroxidase & 0.215028 & 0.5748 & -0.361838 & 0.3472 & -0.453273 & 0.27595 \\
\hline ENSG00000174175 & P-selectin & 0.758977 & 0.2542 & -1.2616 & 0.035 & -1.26137 & 0.05625 \\
\hline ENSG00000116711 & PA24A & 0.253945 & 0.55755 & -1.20128 & 0.007 & -2.08999 & 0.00125 \\
\hline ENSG00000184381 & PA2G6 & 2.83811 & 0.0049 & 3.50727 & 0.00005 & 3.8014 & 0.00005 \\
\hline ENSG00000149269 & PAK1 & 0.184457 & 0.58715 & 0.554972 & 0.1074 & 0.320026 & 0.3308 \\
\hline ENSG00000180370 & PAK2 & 0.0139468 & 0.9761 & -0.652408 & 0.191 & -0.826735 & 0.13585 \\
\hline ENSG00000005381 & PERM & 0.103196 & 0.84875 & 0.0038075 & 0.99585 & -0.866344 & 0.11665 \\
\hline ENSG00000138308 & PG12B & -1.56865 & 1 & 1.23723 & 1 & 1.1968 & 1 \\
\hline ENSG00000121879 & PI3K cat class IA & 0.205456 & 0.77335 & -0.755579 & 0.2899 & 0.305876 & 0.6754 \\
\hline ENSG00000121879 & $\begin{array}{l}\text { PI3K cat class IA (p110- } \\
\text { alpha) }\end{array}$ & 0.205456 & 0.77335 & -0.755579 & 0.2899 & 0.305876 & 0.6754 \\
\hline ENSG00000051382 & $\begin{array}{l}\text { PI3K cat class IA (p110- } \\
\text { beta) }\end{array}$ & 0.0572675 & 0.88035 & -0.077703 & 0.83165 & -0.313535 & 0.38125 \\
\hline ENSG00000171608 & $\begin{array}{l}\text { PI3K cat class IA (p110- } \\
\text { delta) }\end{array}$ & -0.173014 & 0.60385 & -1.013 & 0.00495 & -0.900246 & 0.012 \\
\hline ENSG00000145675 & PI3K reg class IA & 0.211027 & 0.69585 & -0.252244 & 0.65605 & 0.199538 & 0.70535 \\
\hline $\begin{array}{l}\text { ENSG00000117461; } \\
\text { ENSG00000278139 }\end{array}$ & $\begin{array}{l}\text { PI3K reg class IA (p55- } \\
\text { gamma) }\end{array}$ & 0.178026 & 1 & 0.585365 & 1 & 14.7034 & 1 \\
\hline ENSG00000145675 & PI3K reg class IA (p85) & 0.211027 & 0.69585 & -0.252244 & 0.65605 & 0.199538 & 0.70535 \\
\hline
\end{tabular}




\begin{tabular}{|c|c|c|c|c|c|c|c|}
\hline \multirow[b]{2}{*}{ Input IDs } & \multirow[b]{2}{*}{ Network Object Name } & \multicolumn{2}{|c|}{1 hour } & \multicolumn{2}{|c|}{3 hour } & \multicolumn{2}{|c|}{6 hour } \\
\hline & & $\begin{array}{l}\text { Log2Fold } \\
\text { Change }\end{array}$ & p-value & $\begin{array}{l}\text { Log2Fold } \\
\text { Change }\end{array}$ & p-value & $\begin{array}{l}\text { Log2Fold } \\
\text { Change }\end{array}$ & p-value \\
\hline ENSG00000145675 & $\begin{array}{l}\text { PI3K reg class IA (p85- } \\
\text { alpha) }\end{array}$ & 0.211027 & 0.69585 & -0.252244 & 0.65605 & 0.199538 & 0.70535 \\
\hline $\begin{array}{l}\text { ENSG00000105647; } \\
\text { ENSG00000268173 }\end{array}$ & $\begin{array}{l}\text { PI3K reg class IA ( } \mathrm{p} 85- \\
\text { beta) }\end{array}$ & 1.46031 & 0.26735 & 1.69027 & 0.1937 & 2.09423 & 0.1278 \\
\hline ENSG00000101333 & PIB4 & -15.5693 & 1 & 0.278206 & 1 & 1.61796 & 1 \\
\hline ENSG00000154229 & PKC-alpha & 1.46251 & 1 & 1.31425 & 1 & 0.964295 & 1 \\
\hline ENSG00000166501 & PKC-beta & -0.026308 & 0.9484 & -0.464389 & 0.27455 & -0.237803 & 0.5807 \\
\hline ENSG00000163932 & PKC-delta & -0.006682 & 0.9835 & 0.907419 & 0.0052 & 0.385075 & 0.2377 \\
\hline ENSG00000067606 & PKC-zeta & -0.239774 & 0.5907 & -1.19652 & 0.0118 & -1.3945 & 0.00325 \\
\hline ENSG00000069764 & PLA2 & -0.049767 & 1 & -0.496008 & 1 & -0.257958 & 1 \\
\hline ENSG00000069764 & PLA2G10 & -0.049767 & 1 & -0.496008 & 1 & -0.257958 & 1 \\
\hline ENSG00000123739 & PLA2G12 & 0.859792 & 0.40245 & -2.39237 & 1 & -1.85832 & 1 \\
\hline ENSG00000117215 & PLA2G2D & 14.5678 & 1 & 15.6737 & 1 & 14.7034 & 1 \\
\hline ENSG00000100078 & PLA2G3 & 0 & 1 & 15.6737 & 1 & 14.7034 & 1 \\
\hline ENSG00000105499 & PLA2G4C & 1.65877 & 0.00005 & 3.85692 & 0.00005 & 4.54325 & 0.00005 \\
\hline ENSG00000182621 & PLC-beta & 0.320995 & 0.55995 & 0.0635368 & 0.91035 & -0.749371 & 0.16965 \\
\hline ENSG00000182621 & PLC-beta1 & 0.320995 & 0.55995 & 0.0635368 & 0.91035 & -0.749371 & 0.16965 \\
\hline ENSG00000137841 & PLC-beta2 & -0.617107 & 0.1306 & -1.58707 & 0.00005 & -2.59408 & 0.00005 \\
\hline ENSG00000149782 & PLC-beta3 & -0.41557 & 0.38635 & 0.297363 & 0.50225 & 0.0303082 & 0.9445 \\
\hline ENSG00000075651 & PLD1 & 0.655048 & 0.2572 & 2.88132 & 0.00005 & 2.86643 & 0.00005 \\
\hline ENSG00000129219 & PLD2 & -0.420784 & 0.36975 & -1.13418 & 0.02485 & -1.84026 & 0.0017 \\
\hline ENSG00000186951 & PPAR-alpha & -0.263617 & 0.63005 & 0.456419 & 0.449 & 0.30982 & 0.57365 \\
\hline ENSG00000124126 & PREX1 & -0.044813 & 0.88785 & 0.193453 & 0.5318 & 0.25828 & 0.40825 \\
\hline ENSG00000110876 & \begin{tabular}{|l|} 
PSGL-1 \\
\end{tabular} & -0.636731 & 0.0715 & -1.4926 & 0.00005 & -1.76971 & 0.00005 \\
\hline ENSG00000067900 & ROCK & 0.0737406 & 0.82895 & -0.68744 & 0.03295 & -0.369869 & 0.2781 \\
\hline ENSG00000067900 & ROCK1 & 0.0737406 & 0.82895 & -0.68744 & 0.03295 & -0.369869 & 0.2781 \\
\hline
\end{tabular}




\begin{tabular}{|c|c|c|c|c|c|c|c|}
\hline \multirow[b]{2}{*}{ Input IDs } & \multirow[b]{2}{*}{ Network Object Name } & \multicolumn{2}{|c|}{1 hour } & \multicolumn{2}{|c|}{3 hour } & \multicolumn{2}{|c|}{6 hour } \\
\hline & & $\begin{array}{c}\text { Log2Fold } \\
\text { Change }\end{array}$ & p-value & $\begin{array}{l}\text { Log2Fold } \\
\text { Change }\end{array}$ & p-value & $\begin{array}{l}\text { Log2Fold } \\
\text { Change }\end{array}$ & p-value \\
\hline ENSG00000134318 & ROCK2 & 0.228265 & 0.60285 & -0.493979 & 0.3389 & -0.3473 & 0.4248 \\
\hline ENSG00000136238 & Rac1 & -0.058633 & 0.89405 & 0.336282 & 0.4181 & 0.142861 & 0.73685 \\
\hline ENSG00000128340 & Rac2 & -0.215299 & 0.5043 & 0.527082 & 0.09755 & 0.35238 & 0.2695 \\
\hline ENSG00000160271 & RalGDS & 1.38866 & 0.0004 & 2.56647 & 0.00005 & 2.03533 & 0.00005 \\
\hline ENSG00000067560 & RhoA & 0.181352 & 0.6135 & 0.393 & 0.24965 & -0.143086 & 0.7032 \\
\hline ENSG00000196218 & Ryanodine receptor 1 & 1.73729 & 1 & 0.477101 & 1 & 1.04811 & 1 \\
\hline ENSG00000092531 & SNAP-23 & 0.0824211 & 0.86715 & -0.476283 & 0.29665 & 0.236652 & 0.60175 \\
\hline ENSG00000185338 & SOCS1 & -0.443341 & 0.63635 & 0.356888 & 0.62995 & 2.23929 & 0.009 \\
\hline ENSG00000156735 & SODD & -0.259028 & 0.69995 & -1.92762 & 0.01045 & -0.945209 & 0.15885 \\
\hline ENSG00000168610 & STAT3 & 0.318497 & 0.2861 & -0.182545 & 0.5424 & -0.518398 & 0.0822 \\
\hline ENSG00000106089 & Syntaxin 1A & -1.49897 & 0.00725 & -3.17114 & 0.00005 & -3.77 & 0.00005 \\
\hline ENSG00000103496 & Syntaxin 4 & 0.915306 & 0.02265 & 1.76622 & 0.00005 & 1.55602 & 0.00005 \\
\hline ENSG00000135823 & Syntaxin 6 & -0.697353 & 0.1095 & -1.36091 & 0.00095 & -1.18491 & 0.0033 \\
\hline ENSG00000079950 & Syntaxin 7 & 0.0715859 & 0.92885 & 0.412041 & 0.5591 & 1.35408 & 0.05435 \\
\hline ENSG00000067182 & TNF-R1 & -0.474593 & 0.3514 & -0.90111 & 0.04775 & -1.00755 & 0.06335 \\
\hline ENSG00000028137 & TNF-R2 & -0.1768 & 0.5946 & 1.24355 & 0.00035 & 0.729701 & 0.03695 \\
\hline ENSG00000232810 & TNF-alpha & 4.0306 & 0.00005 & 2.77055 & 0.00005 & 1.15677 & 0.00165 \\
\hline ENSG00000226979 & TNF-beta & 0.273582 & 0.5725 & 1.24517 & 0.00705 & 0.212522 & 0.6612 \\
\hline ENSG00000102871 & TRADD & -0.849765 & 0.05665 & -1.15573 & 0.0059 & -0.85951 & 0.0678 \\
\hline ENSG00000127191 & TRAF2 & -0.070513 & 0.94135 & 0.0796205 & 0.93035 & 0.548455 & 0.5636 \\
\hline ENSG00000131323 & TRAF3 & 0.0651305 & 0.87575 & 2.40231 & 0.00005 & 2.67965 & 0.00005 \\
\hline $\begin{array}{l}\text { ENSG00000263620; } \\
\text { ENSG00000220205 }\end{array}$ & VAMP2 & -0.26636 & 0.79795 & -1.81689 & 0.57355 & 1.47089 & 0.0633 \\
\hline ENSG00000141968 & VAV-1 & -0.055979 & 0.8732 & 1.09768 & 0.0008 & 0.702089 & 0.03485 \\
\hline ENSG00000134215 & VAV-3 & 0.187493 & 0.6775 & 0.512374 & 0.2545 & -0.061412 & 0.896 \\
\hline
\end{tabular}




\begin{tabular}{|c|c|c|c|c|c|c|c|}
\hline \multirow[b]{2}{*}{ Input IDs } & \multirow[b]{2}{*}{ Network Object Name } & \multicolumn{2}{|c|}{1 hour } & \multicolumn{2}{|c|}{3 hour } & \multicolumn{2}{|c|}{6 hour } \\
\hline & & $\begin{array}{l}\text { Log2Fold } \\
\text { Change }\end{array}$ & p-value & $\begin{array}{l}\text { Log2Fold } \\
\text { Change }\end{array}$ & p-value & $\begin{array}{l}\text { Log2Fold } \\
\text { Change }\end{array}$ & p-value \\
\hline ENSG00000100568 & VTI1B & -0.23661 & 0.7201 & -1.13439 & 0.0526 & -0.284376 & 0.6871 \\
\hline ENSG00000169896 & alpha-M/beta-2 integrin & -0.072381 & 0.8493 & -0.199032 & 0.59635 & -0.635927 & 0.10645 \\
\hline ENSG00000177606 & c-Jun & -0.495397 & 0.1884 & -0.793995 & 0.03005 & -0.218374 & 0.55605 \\
\hline ENSG00000175592 & c-Jun/Fra-1 & 0.700587 & 0.10625 & -1.95565 & 0.00005 & -2.27314 & 0.00005 \\
\hline ENSG00000170345 & c-Jun/c-Fos & 0.540993 & 0.1754 & -1.63712 & 0.00005 & -0.447981 & 0.2341 \\
\hline ENSG00000177606 & c-Jun/c-Jun & -0.495397 & 0.1884 & -0.793995 & 0.03005 & -0.218374 & 0.55605 \\
\hline ENSG00000132155 & c-Raf-1 & 0.127627 & 0.7162 & -0.041701 & 0.8997 & 0.248827 & 0.4648 \\
\hline ENSG00000116711 & cPLA2 & 0.253945 & 0.55755 & -1.20128 & 0.007 & -2.08999 & 0.00125 \\
\hline ENSG00000165168 & gp91-phox & 0.530715 & 0.25785 & 1.60592 & 0.00045 & 1.62704 & 0.00055 \\
\hline ENSG00000007171 & iNOS & -15.5693 & 1 & -12.529 & 1 & 2.07421 & 1 \\
\hline ENSG00000051523 & p22-phox & -0.234277 & 0.64875 & 0.438144 & 0.3666 & 0.201541 & 0.6868 \\
\hline ENSG00000185386 & p38 MAPK & -2.27641 & 1 & -0.431726 & 1 & -1.38824 & 1 \\
\hline ENSG00000112062 & p38alpha (MAPK14) & -0.02206 & 0.96605 & -0.716459 & 0.1727 & -1.45325 & 0.006 \\
\hline ENSG00000185386 & p38beta (MAPK11) & -2.27641 & 1 & -0.431726 & 1 & -1.38824 & 1 \\
\hline ENSG00000156711 & p38delta (MAPK13) & -0.134623 & 0.79435 & 0.596537 & 0.19465 & 0.422063 & 0.37635 \\
\hline ENSG00000188130 & p38gamma (MAPK12) & 0.45834 & 1 & 0.110895 & 1 & -0.029106 & 1 \\
\hline ENSG00000100365 & p40-phox & 0.05836 & 0.8683 & -0.397913 & 0.2401 & 0.112339 & 0.7651 \\
\hline ENSG00000116701 & p67-phox & -0.011738 & 0.97455 & 0.542507 & 0.11335 & 0.447425 & 0.1847 \\
\hline ENSG00000015475 & tBid & 0.106129 & 0.8675 & 1.5263 & 0.0072 & 0.85196 & 0.169 \\
\hline
\end{tabular}


Table 2.4: Fold change of Components of NADPH Oxidase Complex compared to basal

\begin{tabular}{|c|c|c|c|c|}
\hline $\begin{array}{l}\text { ENSEMBL } \\
\text { GENE }\end{array}$ & GENE SYMBOL|DESCRIPTION & $1 \mathrm{hr}$ & 3 hr & $6 \mathrm{hr}$ \\
\hline ENSG00000165168 & CYBB|cytochrome b-245, beta polypeptide & 1.4 & $3.04 *$ & $3.09 *$ \\
\hline ENSG00000051523 & $\begin{array}{l}\text { CYBA|cytochrome b-245, alpha } \\
\text { polypeptide }\end{array}$ & 0.9 & 1.4 & 1.1 \\
\hline ENSG00000158517 & NCF1|neutrophil cytosolic factor 1 & 1.3 & 1.2 & $0.32 *$ \\
\hline ENSG00000116701 & NCF2|neutrophil cytosolic factor 2 & 1.0 & 1.5 & 1.4 \\
\hline ENSG00000100365 & NCF4|neutrophil cytosolic factor 4, 40kDa & 1.0 & 0.8 & 1.1 \\
\hline ENSG00000128340 & $\begin{array}{l}\text { RAC2|ras-related C3 botulinum toxin } \\
\text { substrate } 2 \text { (rho family, small GTP binding } \\
\text { protein Rac2) }\end{array}$ & 0.9 & 1.4 & 1.3 \\
\hline ENSG00000116473 & $\begin{array}{l}\text { RAP1A|RAP1A, member of RAS } \\
\text { oncogene family }\end{array}$ & 1.1 & 0.8 & 0.9 \\
\hline
\end{tabular}

Note: Asterisk denotes $\mathrm{p}$ value $<0.05$ compared to basal unstimulated conditions

Table 2.5: KEGG pathways significantly enriched for differentially expressed genes during challenge with $F$. alocis

\begin{tabular}{|c|l|l|l|}
\hline Time Point & DEG Count & Description & P-value \\
\hline & 19 & Cytokine-cytokine receptor interaction & $5.40 \mathrm{E}-09$ \\
\hline & 13 & TNF signaling pathway & $2.50 \mathrm{E}-08$ \\
\hline & 10 & Rheumatoid arthritis & $3.50 \mathrm{E}-06$ \\
\hline \multirow{3}{*}{$1 \mathrm{~h}$} & 9 & NF-kappa B signaling pathway & $2.70 \mathrm{E}-05$ \\
\cline { 2 - 4 } & 8 & Salmonella infection & $1.50 \mathrm{E}-04$ \\
\hline & 6 & Legionellosis & $9.20 \mathrm{E}-04$ \\
\hline & 6 & NOD-like receptor signaling pathway & $1.10 \mathrm{E}-03$ \\
\hline & 9 & Transcriptional mis-regulation in cancer & $2.40 \mathrm{E}-03$ \\
\hline & 9 & Chemokine signaling pathway & $4.60 \mathrm{E}-03$ \\
\hline & 10 & MAPK signaling pathway & $9.10 \mathrm{E}-03$ \\
\hline \multirow{3}{*}{$3 \mathrm{~h}$} & 26 & Rheumatoid arthritis & $2.30 \mathrm{E}-08$ \\
\hline & 19 & Legionellosis & $1.50 \mathrm{E}-07$ \\
\hline & 29 & Lysosome & $4.10 \mathrm{E}-07$ \\
\hline & 26 & TNF signaling pathway & $1.40 \mathrm{E}-06$ \\
\hline $3 \mathrm{~h}$ & 18 & NOD-like receptor signaling pathway & $1.50 \mathrm{E}-06$ \\
\hline
\end{tabular}




\begin{tabular}{|c|c|c|c|}
\hline & 22 & NF-kappa B signaling pathway & $5.70 \mathrm{E}-06$ \\
\hline & 40 & Cytokine-cytokine receptor interaction & $4.00 \mathrm{E}-05$ \\
\hline & 17 & Epithelial cell signaling in Helicobacter pylori infection & $8.50 \mathrm{E}-05$ \\
\hline & 19 & Salmonella infection & $1.20 \mathrm{E}-04$ \\
\hline & 22 & Toll-like receptor signaling pathway & $1.30 \mathrm{E}-04$ \\
\hline & 30 & Chemokine signaling pathway & $6.40 \mathrm{E}-04$ \\
\hline & 20 & Chagas disease (American trypanosomiasis) & $8.00 \mathrm{E}-04$ \\
\hline & 13 & Vibrio cholerae infection & $9.00 \mathrm{E}-04$ \\
\hline & 28 & Influenza A & $1.00 \mathrm{E}-03$ \\
\hline & 23 & Measles & $1.30 \mathrm{E}-03$ \\
\hline & 34 & Endocytosis & $2.70 \mathrm{E}-03$ \\
\hline & 24 & Phagosome & $2.80 \mathrm{E}-03$ \\
\hline & 13 & Apoptosis & 4.40E-03 \\
\hline & 20 & Epstein-Barr virus infection & $5.30 \mathrm{E}-03$ \\
\hline & 13 & Inflammatory bowel disease (IBD) & $5.70 \mathrm{E}-03$ \\
\hline & 17 & Inflammatory mediator regulation of TRP channels & $6.40 \mathrm{E}-03$ \\
\hline & 26 & Herpes simplex infection & $8.70 \mathrm{E}-03$ \\
\hline & 10 & Sphingolipid metabolism & 0.014 \\
\hline & 12 & Pancreatic cancer & 0.017 \\
\hline & 32 & HTLV-I infection & 0.018 \\
\hline & 27 & Viral carcinogenesis & 0.019 \\
\hline \multirow{17}{*}{$6 \mathrm{~h}$} & 22 & Legionellosis & $2.70 \mathrm{E}-07$ \\
\hline & 46 & Chemokine signaling pathway & $1.20 \mathrm{E}-06$ \\
\hline & 20 & Apoptosis & $1.80 \mathrm{E}-05$ \\
\hline & 30 & Lysosome & $1.20 \mathrm{E}-04$ \\
\hline & 32 & Measles & $1.20 \mathrm{E}-04$ \\
\hline & 37 & Influenza A & $4.40 \mathrm{E}-04$ \\
\hline & 24 & Estrogen signaling pathway & $9.10 \mathrm{E}-04$ \\
\hline & 22 & Rheumatoid arthritis & $1.00 \mathrm{E}-03$ \\
\hline & 40 & Viral carcinogenesis & $1.40 \mathrm{E}-03$ \\
\hline & 16 & NOD-like receptor signaling pathway & $1.60 \mathrm{E}-03$ \\
\hline & 45 & Endocytosis & $1.80 \mathrm{E}-03$ \\
\hline & 29 & Insulin signaling pathway & $2.50 \mathrm{E}-03$ \\
\hline & 24 & TNF signaling pathway & $2.70 \mathrm{E}-03$ \\
\hline & 26 & Sphingolipid signaling pathway & $2.90 \mathrm{E}-03$ \\
\hline & 8 & Other glycan degradation & $3.00 \mathrm{E}-03$ \\
\hline & 26 & Epstein-Barr virus infection & $3.60 \mathrm{E}-03$ \\
\hline & 17 & Epithelial cell signaling in Helicobacter pylori infection & $4.00 \mathrm{E}-03$ \\
\hline $6 \mathrm{~h}$ & 24 & Toxoplasmosis & 4.00E-03 \\
\hline
\end{tabular}




\begin{tabular}{|l|l|l|l|}
\hline 23 & Chagas disease (American trypanosomiasis) & $4.10 \mathrm{E}-03$ \\
\hline 23 & Toll-like receptor signaling pathway & $5.20 \mathrm{E}-03$ \\
\hline 13 & Sphingolipid metabolism & $6.80 \mathrm{E}-03$ \\
\hline 16 & Pancreatic cancer & $7.30 \mathrm{E}-03$ \\
\hline 33 & Tuberculosis & $8.20 \mathrm{E}-03$ \\
\hline 21 & Phosphatidylinositol signaling system & $9.10 \mathrm{E}-03$ \\
\hline 21 & Inflammatory mediator regulation of TRP channels & $9.10 \mathrm{E}-03$ \\
\hline 14 & Non-small cell lung cancer & 0.011 \\
\hline 17 & Pertussis & 0.012 \\
\hline 19 & Prostate cancer & 0.013 \\
\hline 13 & Vibrio cholerae infection & 0.015 \\
\hline 25 & Platelet activation & 0.016 \\
\hline 16 & Leishmaniasis & 0.016 \\
\hline 27 & Hepatitis B & 0.018 \\
\hline 11 & Bladder cancer & 0.018 \\
\hline
\end{tabular}




\section{CHAPTER 3:}

\section{THE TLR2 TANGO: FILIFACTOR ALOCIS MANIPULATES NEUTROPHIL SIGNALING TO PREVENT KILLING WHILE PROMOTING INFLAMMATION}

\section{Introduction}

One of the primary roles of neutrophils is host defense against microbial challenge. However, their efficient antimicrobial activity hinges on the recognition of microbial invaders and firing of the complex intracellular signal transduction pathways that link neutrophil functional processes [325]. This process is initiated through the ligation of cell surface receptors called pattern recognition receptors (PRR), which recognize general microbial structures. There are several PRR, including C-type lectins, NOD-like, RIG-like, and Toll-like receptors (TLR), but in this thesis the focus will be on TLRs.

Toll-like receptors belong to the IL-1R/TLR family of receptors and are characterized by leucine-rich repeats on their extracellular domains. Out of the 13 TLR members in the family of receptors, TLR 1 to -9 are conserved in mouse and humans, while neutrophils express all tested TLR except TLR3 and 7 [325-330]. Generally, TLR1, -2, -4, -5 and -6 are present on the neutrophil cell surface, while TLR, -8 and -9 are in intracellular endocytic compartments. Regardless of its cellular localization, TLRs mediate the recognition of microbial components defined as pathogen-associated molecular patterns (PAMPs). These microbial components include triacyl lipopeptides (TLR1 ligand), 
peptidoglycan (TLR2 ligand), bacterial lipopolysaccharide (LPS, TLR4 ligand), flagellin (TLR5 ligand), diacyl lipopeptides (TLR6 ligand), bacterial CpG DNA (TLR9 ligand), and viral single-stranded RNA (TLR8 ligand) [331]. TLR4 is regarded as the major receptor for recognizing LPS from Gram-negative bacterial species while TLR2 recognizes the lipopeptides, lipoteichoic acids (LTAs), and peptidoglycans of Gram-positive bacterial species. Albeit at lower levels than monocytes, neutrophils express both TLR2 and -4 on their surface and can upregulate the expression of TLR2 after exposure to several bacterial species $[109,332] . F$. alocis is a non-flagellated, Gram-positive bacterium that has been shown to activate TLR2, but not TLR4 [275].

Once microbial PAMPS are recognized, a complex signal transduction cascade is initiated to elicit antimicrobial and inflammatory functions. As shown in Figure 3.1, once TLR2 binds with its ligand, the adaptor protein MyD88-like adaptor protein (Mal, also called TIRAP) is recruited to the receptor's cytoplasmic tail to facilitate the recruitment and bridge the binding of myeloid differentiation primary-response protein 88 (MyD88) to the TLR2 receptor. Once at the cytoplasmic tail of the receptor, MyD88 forms a complex called the Myddsome with several IL-1 receptor-associated kinase (IRAK) -1, -2 and -4 [333]. IRAK4 is the first recruited to MyD88, where IRAK4 will auto-phosphorylate its central kinase domain and activate IRAK1 and IRAK2 through phosphorylation [334]. The Myddsome assembly induces the association and activation of E3 ubiquitin (Ub) ligase TNF receptor-associated factor 6 (TRAF6), which facilitates the activation of transcription factor, interferon regulatory factor (IRF) -5 [335], or associates with the TGF- $\beta$ activated kinase 1 (TAK1) complex comprised of TAK1, TAK1-binding protein 1 (TAB1), and TAB2/3. Downstream of the TAK1 complex is the initiation of the MAPK and NF- $\kappa \mathrm{B}$ 
pathways, which culminate in the activation of several transcription factors like AP-1, CREB, and NF- $\kappa \mathrm{B}$ (comprising subunits p50 and p65) [334].

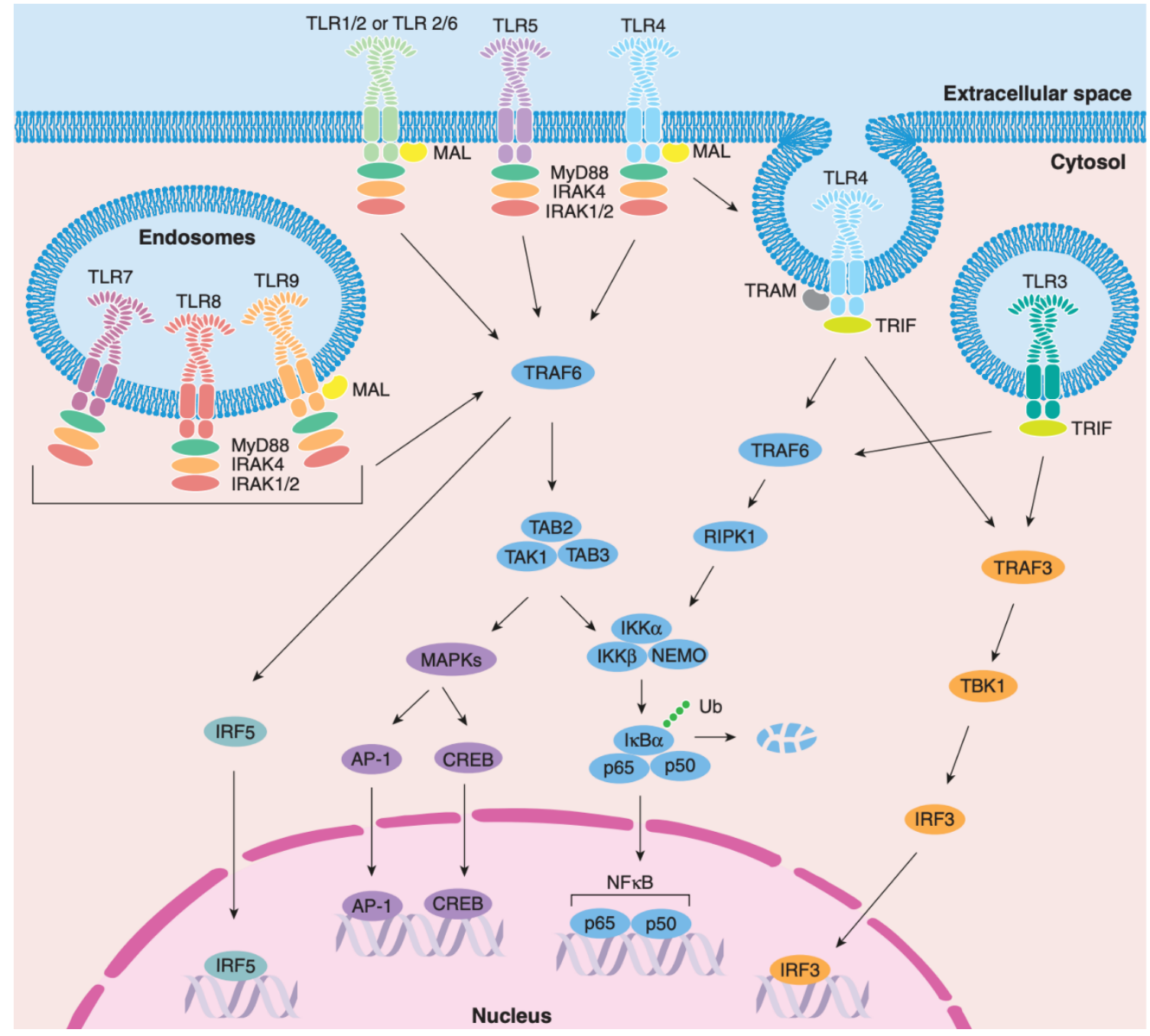

FIGURE 3.1: Toll-like Receptor (TLR) signaling cascades. Adapted from [334].

Activation of TLR2 results in multiple phenotypic and functional changes in neutrophils. First, TLR2 ligation can increase adhesion and chemotaxis in neutrophils. Stimulation of human neutrophils with the TLR2 agonist, $\mathrm{Pam}_{3} \mathrm{CysSerLys}_{4}\left(\mathrm{PAM}_{3} \mathrm{CSK}_{4}\right)$, resulted in the shedding of L-selectin (CD62L), upregulation of CD11b, and downregulation of chemotactic receptors, CXCR2 and CXCR1, on the plasma membrane 
$[327,336]$. This was also the case in our hands, where neutrophil interaction with F. alocis through TLR2 ligation resulted in defects in neutrophil chemotaxis [275]. However, TLR2 agonists also induce neutrophil activation. $\mathrm{PAM}_{3} \mathrm{CSK}_{4}$ stimulation increased phagocytosis of opsonized latex beads, induced ROS production and primed formyl methionyl leucyl phenylalanine (fMLF)-induced ROS production as well [327, 336]. Granule exocytosis can also be induced through ligation of TLR2. Previous publications from our laboratory have also showed that $F$. alocis induces granule exocytosis through ligation of TLR2 [275]. TLR2 agonists also induce the production and release of cytokines and chemokines from neutrophils through the phosphoinositide 3-kinase (PI3K) and the protein kinase B (Akt) signaling pathway [327, 336, 337]. Literature on TLR2 stimulation and apoptosis has yielded conflicting results, but overall, it appears that TLR2 stimulation has some prosurvival effects [338, 339].

For greater versatility and specificity, TLR2 forms heterodimers with TLR1 and TLR6. TLR2/1 recognizes triacylated lipopeptides and TLR2/6 recognizes diacylated lipopeptides and zymosan from Saccharomyces cerevisiae [338, 340, 341]. Experimentally, synthetic and microbe-derived specific ligands for TLR2/1 $\left(\mathrm{PAM}_{3} \mathrm{CSK}_{4}\right)$ and TLR2/6 (FSL-1, MALP-2) are available, but much of the information known about TLR2 signaling in human neutrophils has been conducted with the TLR2/1 agonist. What is known using the TLR2/6 agonist, FSL-1, is that TLR2/6 ligation also increases CD11b expression, induces exocytosis of specific granules, can prime ROS generation and induces cytokine release $[342,343]$. Previous work showed that $F$. alocis activates TLR2/6 in HEK293 cells [253], but this has not been proven in human neutrophils. 
Pathogenic bacteria have evolved elaborate strategies to block intracellular signaling pathways that activate the host immune response. In fact, virtually every step of the TLR2 signaling pathway can be inhibited through bacterially derived proteases, acetyltransferases, kinases/phosphatases, deubiquitinases, proteins that act as TIR mimics, etc. (Reviewed in [344]). Additionally, bacteria have also developed ways to activate signaling pathways at different stages of infection to their advantage. For example, the oral pathogen, $P$. gingivalis, evades clearance by neutrophils and promotes a pro-inflammatory environment by stimulating cross-talk between TLR2/1 and C5aR that results in the degradation of MyD88 [158, 244]. This reroutes signaling through Mal, which activates the PI3K pathway instead of the MAPK pathway and increases the release of proinflammatory cytokines while decreasing the efficiency of neutrophil microbicidal mechanisms. Ultimately, this results in the impaired clearance of systemic bacteria.

Little to nothing is known about the TLR signaling cascade elicited or influenced by $F$. alocis, whose pathogenic potential continues to be defined. However, based on the impaired neutrophil effector functions previously observed, it is clear that $F$. alocis has devised a mechanism to escape killing by the immune system while stimulating inflammation. In this exploratory chapter, I sought to determine the initial steps of neutrophil intracellular signaling after challenge with $F$. alocis, and to determine how the bacteria modulates intracellular signaling to control neutrophil functions. Our data shows that $F$. alocis signals through TLR2/6 in human neutrophils, and that the oral bacterium induces cytokine release through TLR2 and MyD88 dependent pathways. However, $F$. alocis is modulating TLR2 signaling to survive within neutrophils by inhibiting phagosome maturation. Our results are the first to delineate the $F$. alocis strategies to control neutrophil 
effector functions by interrupting intracellular signaling and will enhance our understanding of how oral bacteria undermine the innate immune system.

\section{Materials \& Methods}

Neutrophil isolation: Human donor recruitment, blood draws, and materials required for this procedure were in accordance with the guidelines approved by the Institutional Review Board of the University of Louisville. Neutrophils were isolated from venous blood of healthy donors using plasma-Percoll gradients, as described previously [297].

Mouse strains: Bone marrow neutrophil isolations were completed on legs from male and female mice between 8 to 12 weeks of age. TLR2 ${ }^{-/-}$and C57BL/6 control mice were originally obtained from the Jackson Laboratory and bred in the University of Louisville animal facilities. MyD88 $8^{-/-}$and wild-type albino C57BL/6J mice $\left(\mathrm{B} 6(\mathrm{Cg})-\mathrm{Tyr}^{\mathrm{c}-2 \mathrm{~J}} / \mathrm{J}\right)$ were a gift from Dr. Jonathan Warawa and bred in-house. The procedures used for our animal studies were reviewed and approved by the University of Louisville Institutional Animal Care and Use Committee.

Bone Marrow Neutrophil isolation: Mice were sacrificed by $\mathrm{CO}_{2}$ and the tibia and femur were extracted and cleaned from excess flesh. The ends of each tibia and femur were clipped with dissecting scissors and the bone marrow cells were flushed with PBS supplemented with $1 \%$ glucose and $0.1 \%$ bovine serum albumin (PBS-BG) using a syringe with $22 \mathrm{G}$ needle. The pooled bone marrow was then separated by gentle pipetting with the $22 \mathrm{G}$ needle followed by filtration through a sterile $70-\mu \mathrm{m}$ nylon cell strainer to remove cell 
clumps and bone particles. For assays where cytokines were measured, positive selection was performed using the MACS mouse Anti-Ly-6G MicroBead Kit (Miltenyi Biotec Inc., CA, USA) according to manufacturer's instructions. For all other experiments, a percoll density gradient protocol by the Mócsai laboratory was used $[345,346]$. Briefly, the red blood cells from the bone marrow prep were lysed hypotonically using a $0.2 \% \mathrm{NaCl}$ solution. Osmolarity was restored with $1.6 \% \mathrm{NaCl}$ and after a wash, cells were resuspended in PBS-BG and carefully pipetted on a 62.5\% Percoll gradient and cells were centrifuged for $30 \mathrm{~min}$ at $1000 \mathrm{xg}$, brake off. A loose pellet of neutrophils will form at the bottom of $62.5 \%$ gradient. By flow cytometry analysis after staining for Ly6G and CD11b, 80\% or more of the cells will be neutrophils.

Bacterial strains and growth conditions: F. alocis ATCC 38596 was cultured in brain heart infusion (BHI) broth supplemented $5 \mathrm{mg} / \mathrm{mL}$ yeast extract, L-cysteine $(0.05 \%)$ and arginine $(0.05 \%)$ for 7 days anaerobically at $37{ }^{\circ} \mathrm{C}$ as previously described $[275,276]$. Serum opsonization was performed by incubating F. alocis at $37{ }^{\circ} \mathrm{C}$ for 20 min in $10 \%$ normal human serum (Complement Technology, Inc., Tyler, TX, USA). Heat killed F.alocis was generated by incubation at $90{ }^{\circ} \mathrm{C}$ for $60 \mathrm{~min}$. Non-viability was confirmed by incubation in culture media at same conditions used for the live organism. $P$. gingivalis ATCC 33277 was cultured anaerobically at $37{ }^{\circ} \mathrm{C}$ in trypticase soy broth supplemented with yeast extract $(1 \mathrm{mg} / \mathrm{mL})$, hemin $(5 \mu \mathrm{g} / \mathrm{mL})$ and menadione $(1 \mu \mathrm{g} / \mathrm{mL})$ as previously described [253]. 
Extracellular respiratory burst response: Human neutrophils $\left(4 \times 10^{6}\right.$ cells $\left./ \mathrm{mL}\right)$ were pretreated with $\mathrm{PAM}_{3} \mathrm{CSK}_{4}(1 \mu \mathrm{g} / \mathrm{mL}$, Invivogen), FSL-1 (100 $\mathrm{ng} / \mathrm{mL}$, Invivogen), or opsonized $F$. alocis for $30 \mathrm{mins}$ at $37^{\circ} \mathrm{C}$, followed by stimulation with fMLF (300 nM) for $5 \mathrm{~min}$. After stimulation, the samples were centrifuged for $10 \mathrm{~min}$ at $600 \mathrm{xg}$ and $4{ }^{\circ} \mathrm{C}$, and supernatants were collected. Superoxide anion release was measured spectrophotometrically at $550 \mathrm{~nm}$ as the superoxide dismutase-inhibitable reduction of ferricytochrome c as previously described [297].

Granule exocytosis: Human neutrophils $\left(4 \times 10^{6}\right.$ cells $\left./ \mathrm{ml}\right)$ were unstimulated (basal), pretreated with TLR6 neutralizing antibody (50 ug/mL, Invivogen) or Rat IgG isotype control (50 ug/mL, Invivogen) for 60 minutes and/or TLR2 neutralizing antibody (50 $\mu \mathrm{g} / \mathrm{ml}$; clone TL2.1; BioLegend) or isotype control IgG2a kappa (50 $\mu \mathrm{g} / \mathrm{ml}$; clone MOPC-173; BioLegend) for 30 minutes. Then, cells were stimulated with FSL-1 (100 ng/mL, Invivogen), or $F$. alocis (MOI 10) at $37{ }^{\circ} \mathrm{C}$ for $30 \mathrm{~min}$. Exocytosis of specific granules and secretory vesicles was determined by measuring the increase in plasma membrane expression of membrane-associated receptors using antibodies: FITC-conjugated anti human CD66b (Biolegend, clone G10F5) and PE-conjugated anti-human CD35 (Biolegend, clone E11), respectively. After treatment, the samples were washed with $0.5 \%$ sodium azide, fixed with $1 \%$ paraformaldehyde, and analyzed by flow cytometry using a BD FACSCalibur.

BacLight assay: As previously described [284, 322], the two DNA dyes, membranepermeable Syto9 (stains all bacteria) and membrane-impermeable propidium iodide (PI) 
(stains only nonviable bacteria), were used to determine bacteria viability associated with mouse neutrophils. Bone marrow neutrophils $\left(1 \times 10^{6}\right.$ cells $\left./ \mathrm{mL}\right)$ were allowed to settle on poly-L-lysine-coated coverslips for 30 minutes, followed by challenge with opsonized $F$. alocis (MOI 10) that was centrifuged at $600 \mathrm{x} \mathrm{g}$ for $4 \mathrm{~min}$ at $14{ }^{\circ} \mathrm{C}$ to synchronize phagocytosis. Following challenge of 1 hour in a cell culture incubator at $37^{\circ} \mathrm{C}$, mixed dye solutions (AlexaFluor 670-Phallodin, $5 \mu \mathrm{M}$ Syto9, $30 \mu \mathrm{M}$ PI in 0.1 MOPS (morpholinepropanesulfonic acid) ( $\mathrm{pH} 7.2)-1 \mathrm{mM} \mathrm{MgCl2}$ ) were added to samples. Z-stack confocal images were acquired within 30 min using a Fluoview FV1000 confocal microscope and analyzed by FV-10ASW software. Quantification was performed by counting the total viable and nonviable bacteria both intracellularly from 100 neutrophils in 3 independent experiments.

Phagocytosis assay and Myeloperoxidase staining: Bone marrow neutrophils $\left(1 \times 10^{6}\right.$ cells/mL) were allowed to settle on poly-L-lysine-coated coverslips for 30 minutes, followed by challenge with opsonized $F$. alocis (MOI 10) that was centrifuged at $600 \mathrm{xg}$ for 4 min at $14{ }^{\circ} \mathrm{C}$ to synchronize phagocytosis. After an hour of challenge, cells were fixed with $4 \%$ paraformaldehyde, $10 \mathrm{~min}$ at room temperature. Extracellular bacteria were stained using $F$. alocis antibodies (1:1000) and Alexa Fluor 647-labeled (1:1000) secondary antibodies for 1 hour each and antibodies were fixed in place with $4 \%$ paraformaldehyde for $10 \mathrm{~min}$, room temperature. Cells were permeabilized and blocked with $3 \%$ bovine serum albumin with $0.02 \%$ saponin. Then, intracellular $F$. alocis was stained using F. alocis antibodies (1:1000) and Alexa Fluor 488-labeled (1:1000) secondary antibodies for 1 hour each. For experiments where myeloperoxidase was stained, the same protocol as above was followed, except cells were blocked with $4 \%$ goat serum 
and incubated with a FITC-labeled mouse myeloperoxidase antibody (1:50, Hycult Biotech) overnight. Z-stack confocal images were acquired using a Fluoview FV1000 confocal microscope and analyzed by FV-10ASW software. Quantification for phagocytosis was performed by counting 100 neutrophils and determining the number of neutrophils that had intracellular F. alocis. Quantification for myeloperoxidase was performed by counting 100 infected neutrophils and determining the number of neutrophils that had F. alocis that co-localized $50 \%$ or more with the myeloperoxidase.

F. alocis Colony Forming Units: F. alocis was co-cultured with isolated bone marrow neutrophils at a $\mathrm{MOI}$ of $10 \mathrm{in} \mathrm{RPMI}+5 \%$ fetal bovine serum in a $37^{\circ} \mathrm{C}, 5 \% \mathrm{CO}_{2}$ incubator. At the end of each timepoint, $F$. alocis and neutrophils were centrifuged (6000g, 2 min, 4 $\left.{ }^{\circ} \mathrm{C}\right)$ to pellet all bacteria and immune cells. The pellets were lysed for 1 minute in $0.02 \%$ saponin and serially diluted in sterile PBS. These dilutions were plated in duplicate $10 \mu \mathrm{L}$ drop aliquots on anaerobic BHI plates (BHI media (7.4g/200ml), L-cysteine $(0.1 \mathrm{~g} / 200 \mathrm{ml})$, yeast extract $(1 \mathrm{~g} / 200 \mathrm{ml})$, agar powder $(3 \mathrm{~g} / 200 \mathrm{ml}), 0.05 \%$ arginine and $5 \%$ sheep blood). The plates were transferred into an anaerobic chamber and allowed to grow for 72 hours. Colonies that grew were counted and Gram stained.

Intracellular ROS generation: ROS generation time courses with BMN were monitored by luminol chemiluminescence. Plate $50 \mu \mathrm{L}$ of a $2 \times 10^{6} \mathrm{BMN} / \mathrm{ml}$ stock in triplicate and incubate with luminol $(125 \mu \mathrm{M})$ with superoxide dismutase $(15 \mathrm{nM})$ for 10 minutes. Stimulate with opsonized zymosan $(2 \mathrm{mg} / \mathrm{mL})$, viable or heat-killed $F$. alocis (MOI 10). After a short centrifugation to synchronize phagocytosis, plates were placed in a 
Spectramax L Luminometer (Molecular Devices) and readings were taken every minute for the duration of 90 minutes. Single timepoint measurements of the phagocytosisstimulated respiratory burst response in human neutrophils was measured by $2^{\prime}, 7^{\prime}$ dichlorofluorescein (DCF, $5 \mu \mathrm{M}$ ) and analyzed by a BD FACSCalibur flow cytometer as previously described [284]. In experiments with the MyD88 inhibitor (ST-2825, Med Chem Express), 1-25 $\mu \mathrm{M}$ were tested, but all subsequent experiments used a pre-treatment of $20 \mu \mathrm{M}$ for 60 minutes.

Murine Bone Marrow Neutrophil Lysis \& Western Blotting: After incubation in RPMI$1640+5 \%$ fetal bovine serum or with viable or heat-killed F. alocis (MOI 10) for 4 or 24 hours in a $37{ }^{\circ} \mathrm{C}, 5 \% \mathrm{CO}_{2}$ incubator, $2 \times 10^{6}$ cells $/ \mathrm{mL}$ were lysed directly into $50 \mu \mathrm{L}$ of SDS-sample buffer. $5 \mu \mathrm{L}$ of the total cell lysates were separated by $12 \%$ SDS-PAGE and immunoblotted with antibodies for $F$. alocis (1:1000) or $\beta$-actin (1:1000, Cell Signaling, Danvers, MA, USA). The appropriate secondary antibodies were used at 1:2000 dilution (Cell Signaling, Danvers, MA, USA). The ECL system (Amersham Pharmacia Biotech, Little Chalfont, United Kingdom) or the SuperSignal West Femto Maximum Sensitivity Substrate (Thermo Scientific,Waltham, MA, USA) was used to visualize antigen-antibody reactions. Densitometric values of each band were calculated using Image Lab software (BioRad, Hercules, CA, USA).

Intracellular staining of I $\kappa B \alpha$ : After stimulation with FSL-1 $(100 \mathrm{ng} / \mathrm{mL})$, live or heatkilled opsonied $F$. alocis, human neutrophils $\left(2 \times 10^{6}\right.$ cells $/ \mathrm{mL}$ for each timepoint $)$ were fixed with $4 \%$ paraformaldehyde, permeabilized and blocked with $0.02 \%$ saponin in $3 \%$ 
BSA for 1 hour. Primary antibodies for $\mathrm{I} \kappa \mathrm{B} \alpha(1: 800$, Cell signaling) were incubated with cells overnight followed by 3 washed with PBS. An alexa fluor 488 secondary antibody (1:1000, Life Technologies) or FITC-IgG1 isotype control mAb (1:1000, Cell Signaling) was incubated with the cells for 1 hour. After washing 3 times with PBS, cells were read on a BD FACSCalibur flow cytometer.

Cytokine collection from bone marrow neutrophils: Highly pure $(99 \%+)$ bone marrow neutrophils $\left(1 \times 10^{6}\right.$ cells $\left./ \mathrm{mL}\right)$ were allowed to settle in the wells of a 96 well plate for 30 minutes in RPMI $1640+5 \%$ fetal bovine serum, followed by challenge with opsonized $F$. alocis or non-opsonized $P$. gingivalis (MOI 10) that was centrifuged at $600 \mathrm{x} \mathrm{g}$ for $4 \mathrm{~min}$ at $14{ }^{\circ} \mathrm{C}$ to synchronize phagocytosis. After 24 hours, supernatants were collected from the wells, centrifuged (10000g for $5 \mathrm{~min}$ ) to remove any bacteria or cells and transferred to sterile microcentrifuge tubes with $1 \%$ protease and phosphatase inhibitors. Supernatants were stored at $-80{ }^{\circ} \mathrm{C}$ until analyzed by multiplex assays.

Human Neutrophil Western Blotting: Human neutrophils $\left(10 \times 10^{6}\right.$ cells/mL $)$ were cultured at $37{ }^{\circ} \mathrm{C}, 5 \% \mathrm{CO}_{2}$ in RPMI- $1640+5 \%$ heat inactivated human serum and left unstimulated, stimulated with FSL-1 (100 ng/mL), or challenged with live F. alocis for 3, 6, 10, or 20 hours. Then, cells were centrifuged at $6,000 \times g$ for $30 \mathrm{~s}$ and lysed for $30 \mathrm{~min}$ on ice in icecold lysis buffer (20 mM Tris- $\mathrm{HCl}$ [pH 7.5], $150 \mathrm{mM} \mathrm{NaCl,1 \%}$ [vol/vol] Triton X-100, 0.5\% [vol/vol] Nonidet P-40, 20 mM NaF, 20 mM NaVO3, 1 mM EDTA, 1 mM EGTA, $5 \mathrm{mM}$ phenylmethylsulfonyl fluoride [PMSF], $21 \mu \mathrm{g} / \mathrm{ml}$ aprotinin, $5 \mu \mathrm{g} / \mathrm{ml}$ leupeptin, and 4mM Diisopropyl fluorophosphates [DFP]). After protein estimation using the Pierce BCA 
protein assay kit (Thermo Scientific, Waltham, MA, USA), samples were adjusted to a concentration of $2 \mu \mathrm{g} / \mu \mathrm{L}$. Unless otherwise noted, $20 \mu \mathrm{g} / \mu \mathrm{L}$ of total cell lysates were separated by $12 \%$ SDS-PAGE and immunoblotted with antibodies for MyD88, phosphorylated IRAK4, total IRAK4, and $\beta$-actin (1:1000, Cell Signaling, Danvers, MA, USA). The appropriate secondary antibodies were used at 1:2000 dilution (Cell Signaling, Danvers, MA, USA). The ECL system (Amersham Pharmacia Biotech, Little Chalfont, United Kingdom) or the SuperSignal West Femto Maximum Sensitivity Substrate (Thermo Scientific,Waltham, MA, USA) was used to visualize antigen-antibody reactions. Densitometric values of each band were calculated using Image Lab software (BioRad, Hercules, CA, USA).

\section{$\underline{\text { Results }}$}

F. alocis challenge induces differential gene expression in the TLR2 pathway

Using the Metacore software, a list of genes involved in the TLR2/TLR4 signaling pathway was compiled to assess the pattern of differential gene expression (Figure 3.2 A). The expression of two genes (iNOS and TAB2) was downregulated and upregulated so much that they were plotted separately (Figure 3.2 B). Overall, there is a pervasive downregulation of genes in this pathway. Many of the signaling intermediates from the MAPK pathway like ERK1/2, p38, NIK, and MEK4 and -6 show downregulation. Moreover, proteins involved in the initial signaling mechanisms of TLR were also downregulated, like TLR6 and 1, Mal, MyD88, and Tab 1. Out of the proteins that were upregulated, many of them relate to inflammatory processes like IL-12beta, IL-6, IRF5, $\mathrm{NF}-\mathrm{\kappa B}$, and $\mathrm{TNF} \alpha$. It is interesting that $\mathrm{TAB} 2$ is so highly upregulated because in addition 
to its role in the transduction of TLR2 signaling, it is also involved in the pathway that senses bacterial double stranded DNA [347]. The inducible nitric oxide synthase (iNOS) system is more developed in mice than in humans, but it can be found in human neutrophils in azurophilic granules and phagosomes, and have been proposed to have roles as signaling molecules and in bacterial killing [348-351]. In our dataset the expression of iNOS was increased over the time course tested, but the biological significance of this increase in gene expression remains to be examined. Taken together, I conclude that challenge of human neutrophils with $F$. alocis results in significant changes to the expression of components in the TLR2 signaling pathway.

\section{Human neutrophils recognize F. alocis through TLR $2 / 6$}

Previous work from our laboratory showed that $F$. alocis activates TLR2/6 in HEK293 cells [253], but this has not been demonstrated in human neutrophils yet. Neutrophils can undergo a sequential activation process called priming that results in phenotypic changes enhancing the cell functional responses [352]. Whitmore et al. noted that while stimulation with FSL-1, a TLR2/6 ligand, could prime the neutrophils from all their human donors, only a subset of these donors could be primed with the TLR2/1 agonist, $\mathrm{Pam}_{3} \mathrm{CSK}_{4}$ due to a single nucleotide polymorphism on TLR1 [342]. Based on this work, I compared the priming capabilities of $F$. alocis in donors who had low or high priming response to $\mathrm{Pam}_{3} \mathrm{CSK}_{4}$ (Figure 3.3A). Priming was measured as the ability to generate extracellular superoxide by the colorimetric reduction of ferricytochrome $\mathrm{c}$ as previously described [353]. As Whitmore reported, in our hands, $\mathrm{Pam}_{3} \mathrm{CSK}_{4}$ only primed a subset of donors, which are color coded as high priming, whereas FSL-1 could prime all donors. 
Neutrophils primed by F. alocis produced a significantly higher superoxide concentration than $\mathrm{Pam}_{3} \mathrm{CSK}_{4}$ low primers, suggesting that $F$. alocis is recognized through TLR2/6, like FSL-1 (Figure 3.3A). Notably, F. alocis-primed neutrophils still produced significantly lower amounts of superoxide than the FSL-1-primed neutrophils, which could be due to the affinity of the receptor for either one of the ligands and the strength of subsequent downstream signaling. To strengthen this observation, I tested granule exocytosis, which is another neutrophil function that has been linked to TLR2 signaling [275, 342, 343]. Human neutrophils were pre-treated with media, neutralizing antibodies for TLR2 and -6, or corresponding antibody isotype controls, followed by stimulation with FSL-1 or $F$. alocis. When the release of secretory vesicles (Figure 3.3B) and specific granules (Figure 3.3C) was assessed, both FSL-1 and F. alocis stimulated exocytosis, but this was significantly decreased when the cells were pre-treated with the neutralizing antibodies for TLR2/6 while the isotype control had no effect. In conclusion, this data strongly suggests that human neutrophils use the heterodimer receptors TLR2/6 to recognize F. alocis, and that $F$. alocis-induced neutrophil degranulation depends on TLR2/6 activation.

\section{F. alocis modulates TLR2 signaling to survive within neutrophils}

Data published by our group as well as the data presented in this thesis so far, strongly indicate that $F$. alocis can manipulate neutrophil functional responses. Thus, I wanted to assess if $F$. alocis was modulating the TLR2 signaling pathway to interrupt neutrophil functions and favor its survival. To do this, the functional responses were compared between bone marrow neutrophils $(\mathrm{BMN})$ from WT or TLR2 $2^{-{ }_{-}}$mice. 
First, the microbicidal capacity of these BMN was assessed using several methods. The first of these, BacLight, is a confocal microscopy-based assay that uses the dyes Syto9 to label all bacteria and propidium iodide to label dead bacteria that have compromised cell membranes as previously shown in neutrophils [284, 322]. Figure 3.4A shows representative images from the BacLight assay on WT or TLR2 ${ }^{-/-} \mathrm{BMN}$ that were challenged with $F$. alocis for 1 hour. Like the data observed in human neutrophils, BMN were also not able to completely eliminate intracellular $F$. alocis within the first hour of challenge (Figure 3.4B). Moreover, TLR2 ${ }^{-/-}$BMN were able to kill F. alocis slightly better than their WT counterparts (15-20\% decrease), suggesting that $F$. alocis is manipulating TLR2 signaling to remain viable in neutrophils. Notably, this difference was not due to discrepancies in phagocytosis between the mouse genotypes (Figure 3.4C-D). Using $F$. alocis antibodies to differentiate between intracellular (green) or extracellular (magenta/white) bacteria (Figure 3.4C), there was no difference in the number of BMN that phagocytized F. alocis (Figure 3.4D). The disparity in F. alocis killing was also tested by colony forming units (CFU) (Figure 3.4E). After 1 hour of challenge, the $F$. alocis that was cultured with TLR2 ${ }^{-/-}$BMN produced significantly fewer CFU than the bacteria cultured with WT cells. By 4 hours, TLR2 ${ }^{-/-}$BMN still had slightly fewer CFU, but the difference between the genotypes was not statistically significant. This data demonstrates that TLR2 is not involved in the phagocytosis of $F$. alocis, which is to be expected since TLR2 is not a phagocytic receptor. The data also points to the idea that while TLR2 signaling is required for the maximal intracellular survival of the oral pathogen, but that there are likely other mechanisms at play since the killing capacity of neutrophils was not completely restored in the TLR2 null mice. 


\section{$\underline{\text { TLR2 signaling is involved in phagosome maturation but not ROS generation }}$}

To determine why $\mathrm{TLR}^{-/-} \mathrm{BMN}$ are able to eliminate $F$. alocis more efficiently than WT BMN, differences in neutrophil microbicidal mechanisms were measured in both of these genotypes. Intracellular ROS generation was detected during a time course luminol assay, which is represented in Figure 3.5A. Based on the quantification of the area under the curve, it is possible to observe that similar to human neutrophils, BMN were able to quickly generate ROS in response to opsonized zymosan and heat-killed $F$. alocis, but viable $F$. alocis induced minimal ROS production (Figure 3.5B). This trend was the same, regardless of whether the cells expressed TLR2. This suggests that both the robust ROS generation induced by heat-killed $F$. alocis, as well as the dampened ROS response by the viable bacteria, are independent of TLR2. In the case of opsonized zymosan, host cells recognize the yeast particle through TLR2/6, opsonic receptors, and C-type lectin receptors such as dectin-1[354]. Signaling through these other receptors is responsible for the ROS production observed with zymosan and in the case of $F$. alocis, other PRR may be involved in the ROS response.

Oxygen independent microbicidal mechanisms are characterized by the deposition of granule antimicrobial proteins into the phagosome to establish a hostile, non-physiologic environment. Myeloperoxidase (MPO) is found in azurophilic granules and is a key enzyme in the development of a noxious phagosome due to its catalytic activity. As a measure of phagosome maturation and granule fusion with the phagosome, I evaluated the co-localization of MPO with $F$. alocis phagosomes from WT and TLR2 ${ }^{-/-}$BMN after 1 hour of challenge by confocal microscopy (Figure 3.6A). Fewer than $40 \%$ of F alocis phagosomes in WT BMN co-localized with MPO, and while TLR2 ${ }^{-/-}$BMN had a 
significantly greater number of MPO+F. alocis phagosomes, a majority of phagosomes remained negative for MPO (Figure 3.6B). This partial increase in MPO fusion with the $F$. alocis phagosome may be the reason for the marginal increase in $F$. alocis killing. As a measure of total bacterial degradation, I collected whole cell lysates from WT and TLR2 ${ }^{-1-}$ BMNs that were untreated or challenged with viable or heat-killed $F$. alocis for 4 and 24 hours (Figure 3.6C). The F. alocis antibody did not considerably detect proteins in untreated cells from either WT or TLR2 ${ }^{-/}$BMNs, suggesting that the bands detected by the antibody in the other conditions corresponded solely to $F$. alocis and not homologous sequences in neutrophil proteins. The densitometry analysis of these western blots demonstrated that in WT BMN, there is a time-dependent decrease in F. alocis proteins, indicating degradation of F. alocis (Figure 3.6D). This degradation process started earlier and progressed much faster in WT BMN that were challenged with heat-killed F. alocis, indicating that much like in human neutrophils, viable $F$. alocis is actively delaying the maturation of its phagosome in BMNs. In TLR2 $2^{-/}$BMNs, there was more degradation of F. alocis proteins at the earlier timepoint tested, but by 24 hours WT and TLR2 ${ }^{-/}$BMN had comparable levels of $F$. alocis proteins. The enhanced degradation of heat-killed $F$. alocis compared to viable bacteria was similar between WT and TLR2 ${ }^{-/}$BMN. Taken together, this data demonstrates that viable $F$. alocis requires TLR2 signaling to actively delay the recruitment of azurophilic granules to its phagosome.

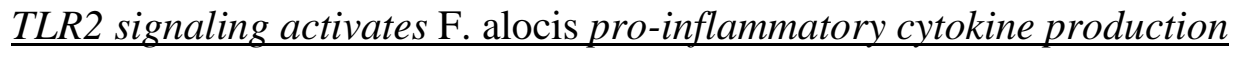

TLR2 stimulation results in the activation of the MAPK and NF-KB pathways. Although F. alocis activation of MAPK through TLR2 and the production of cytokines in 
response to $F$. alocis has been shown [253, 275], the activation of NF-אB or the role TLR2 plays in cytokine production had not been determined. The activation of NF-kB was determined indirectly by measuring the amount of I $\mathrm{KB} \alpha$. In naïve cells, the inhibitory $\mathrm{I} \kappa \mathrm{B} \alpha$ is bound to $\mathrm{NF}-\kappa \mathrm{B}$ to prevent its inadvertent activation. Upon stimulation, I $\mathrm{B} \alpha$ will be phosphorylated, ubiquitinated and degraded by the proteasome, freeing the now active NF- $\kappa \mathrm{B}$ subunits to translocate into the nucleus and perform their effector functions [355]. I $\mathrm{B} \alpha$ was quantified by flow cytometry in human neutrophils that were untreated (basal) or stimulated with FSL-1, F. alocis or heat-killed $F$. alocis for a short time course experiment (Figure 3.7A). Within 15 minutes of stimulation, the cellular stores of I $\mathrm{B} \alpha$ began to be depleted, and by 30 minutes of challenge with all three stimulants, intracellular I $\mathrm{B} \alpha \alpha$ was significantly degraded compared to the basal expression. The kinetics of activation of NF- $\mathrm{KB}$ by F. alocis was comparable to the TLR2/6 agonist, FSL1 , and since $F$. alocis challenge quickly induced IкB $\alpha$ degradation regardless of viability, I conclude that viable $F$. alocis is not inhibiting the activation of NF- $\mathrm{KB}$ at the initial stages of infection.

Subsequently, the role of TLR2 in F. alocis-induced cytokine (Figure 3.7B) and chemokine (Figure 3.7C) production was directly determined by using WT and TLR2 $2^{-/}$ BMN. When challenged with $F$. alocis, WT BMN significantly released all proinflammatory cytokines and the anti-inflammatory cytokine, IL-10 (Figure 3.7B). Similarly, F. alocis induced significant release of chemokines, such as CCL2, CCL3, CCL4 and $\mathrm{KC}$ which are involved in monocyte and neutrophil chemotaxis, respectively by WT BMNs (Fig 3.7C) However, the release of all these cytokines and chemokines was abrogated in TLR2 $2^{--}$conditions, indicating that TLR2 signaling is essential to produce 
cytokines and chemokines in F. alocis infection. In conclusion, this data indicates that $F$. alocis manipulates TLR2 signaling to prevent microbicidal mechanisms, but requires this signaling pathway to induce cytokine and chemokine release.

\section{$\underline{\text { Role of } M y D 88 \text { in antimicrobial responses against } \mathrm{F} \text {. alocis challenge }}$}

MyD88 is the canonical adaptor protein for signaling pathways downstream of TLRs. As a central node of inflammatory pathways and a bottleneck for multiple neutrophil functional responses, it is a prime target for manipulation by pathogens. Burns et al. conducted in vivo infections with $P$. gingivalis that proved that while TLR2-mediated production of pro-inflammatory cytokines was independent of MyD88, MyD88 signaling was required for controlling bacterial load [244]. Thus, I explored the possibility that $F$. alocis was manipulating TLR2 signaling like $P$. gingivalis and assessed the role of MyD88 in F. alocis challenge.

Initially, the role of MyD88 was tested in the phagocytosis of $F$. alocis by confocal microscopy as previously shown. Both WT and MyD88 ${ }^{-/-}$BMN phagocytized the oral pathogen to a similar extent after 1 hour of challenge (Figure 3.8A). Analysis of the killing capacity of these BMN by BacLight demonstrated that $F$. alocis was able to survive equally well in WT and MyD88 ${ }^{-/}$BMN (Figure 3.8B). Around $80 \%$ of the intracellular F. alocis was viable after an hour of culture with WT or MyD88 ${ }^{-/}$BMN (Figure 3.8C). Preliminary experiments by $\mathrm{CFU}$ also confirmed this finding (Figure 3.8D). Together, this data shows that MyD88 is not required for the internalization or intracellular survival of $F$. alocis.

Next, the ROS generation by WT and MyD88 ${ }^{-/-}$BMN was tested. Using a time course luminol based assay, it appeared that there was a slight decrease in MyD88 ${ }^{-/}$BMN's 
ability to produce ROS in response to $F$. alocis (Figure 3.9A). However, when the area under the curve was combined from several mice, there was no significant difference in the ROS generation between mouse genotypes (Figure 3.9B). In human neutrophils, I confirmed this data by optimizing the use of a MyD88 inhibitor (ST2825). Figure 3.9C shows the dose response curve where FSL-1 was used as the agonist and the readout was ROS production. The chosen concentration, $20 \mu \mathrm{M}$, which reduced the FSL-1 ROS production by half, was tested in human neutrophils against viable and heat-killed $F$. alocis (Figure 3.9D). However, pre-treatment of neutrophils with the MyD88 inhibitor had no effect on the minimal production of ROS by viable $F$. alocis. In contrast to the results from MyD88 ${ }^{-/}$BMNs, the MyD88 inhibitor decreased ROS generation by heat-killed $F$. alocis. Overall, this data shows that MyD88 is not involved in the minimal ROS production by viable $F$. alocis.

Since the killing capacity of MyD88 $8^{-/-} \mathrm{BMNs}$ was equal to WT, I expected the $F$. alocis phagosome maturation to be similar between genotypes. By confocal microscopy, after 1 hour of challenge, less than $40 \%$ of $F$. alocis phagosomes had co-localized with myeloperoxidase in both WT and MyD88 ${ }^{-/-}$BMNs (Figure 3.10A-B). This suggests that $F$. alocis does not interfere with neutrophil signaling through MyD88 to delay the maturation of the phagosome at early timepoints. However, MyD88 may be playing a role in phagosome maturation at longer timepoints. The whole cell lysates from WT and MyD88I- BMNs that were challenged with live or heat-killed $F$. alocis for 4 and 24 hours were processed for western blots to determine the degradation of F. alocis (Figure 3.10C). In WT BMN, both viable and heat-killed $F$. alocis were degraded in a time-dependent manner, albeit heat-killed $F$. alocis was degraded to a greater extent (Figure 3.10D). In the 
case of MyD88 $8^{-/-}$BMN, heat-killed $F$. alocis was degraded with similar kinetics as in WT BMN, but viable $F$. alocis in $\mathrm{MyD}^{-/-} \mathrm{BMN}$ was not degraded as much as in WT cells by 24 hours of culture. These results indicate that while MyD88 signaling may not play a role in the initial phagosome maturation, it may be essential to the degradation of the oral pathogen at longer timepoints, though this finding requires more characterization.

Role of MyD88 in inflammatory responses to $\mathrm{F}$. alocis challenge

The dogma in the field is that MyD88 signaling predominantly leads to the production of inflammatory cytokines (e.g., TNF $\alpha$, IL-6, IL1 $\beta$ ) and chemokines (e.g., CC motif ligand 4, CCL4) [356], however, Burns et. al reported that $P$. gingivalis challenge results in MyD88 independent cytokine release [244]. Therefore, I challenged WT and MyD88 ${ }^{-/}$BMNs with $F$. alocis or $P$. gingivalis for 24 hours and measured the resultant cytokine release (Figure 3.11). In WT mice, both $P$. gingivalis and $F$. alocis induced the release of pro-inflammatory cytokines (G-CSF, IL-1 $\beta$, and TNF $\alpha$ ) and chemokines (IL17, CCL2, CCL3, CCL4, and CCL5). Interestingly, F. alocis induced significantly less anti-inflammatory IL-10 than $P$. gingivalis, but greater release of pro-inflammatory immune cell recruitment cytokines like IL-17, CXCL10, CCL3, and CCL4. In our hands, both $F$. alocis and $P$. gingivalis required MyD88 signaling to induce the release of all cytokines and chemokines. In the case of $P$. gingivalis, this could be explained by strain differences between the studies. Therefore, in our system, MyD88 did not play a large role in antimicrobial functions, but it was critical for $F$. alocis-induced inflammation.

F. alocis may be inducing non-canonical MyD88 signaling 
Burns et. al showed that $P$. gingivalis causes the degradation of MyD88 to block MyD88-dependent antimicrobial mechanisms [244], so I tested whether this was taking place in our system by measuring the levels of MyD88 in F. alocis-challenged human neutrophils (Figure 3.12A). The normalized densitometry of MyD88 showed that although levels of MyD88 steadily decreased as the time course progressed, there was no difference in the MyD88 protein expression between untreated (basal) or F. alocis-challenged human neutrophils (Figure 3.12B). Together with our functional analysis of MyD88 ${ }^{-/-} \mathrm{BMNs}$, these results demonstrate that $F$. alocis is not inducing the same mechanism as $P$. gingivalis strain ATCC strains 381 and 53,977. P. gingivalis infection leads to the degradation of MyD88 to inhibit bacterial killing and promotes inflammation through MyD88independent cytokine production. However, F. alocis challenge did not change MyD88 abundance and required MyD88 to be present for cytokine production.

Downstream of MyD88 signaling is the activation of IRAK4 by phosphorylation, a key step in the canonical TLR signaling pathway. To continue evaluating the $F$. alocis activation of TLR2 signaling, western blots for the phosphorylation of IRAK4 were performed on human neutrophils that were stimulated with the TLR2/6 agonist, FSL-1, or F. alocis (Figure 3.12C). FSL-1 stimulation resulted in the transient phosphorylation of IRAK4 that started after 5 min of challenge, and peaked at $30 \mathrm{~min}$. To our surprise, $F$. alocis did not induce phosphorylation at any point in the 90-minute challenge (Figure 3.12D). This response suggests that $F$. alocis is inducing non-canonical signaling from neutrophils, but its full impact on neutrophil functional responses will need more testing.

\section{$\underline{\text { Discussion }}$}


Periodontal pathogens have evolved mechanisms to escape killing by the immune system while stimulating inflammation; however, much of the current literature on the signaling interactions between periodontal pathogens and the innate immune system centers around long-established pathogens such as $P$. gingivalis [357]. The results shown here characterize how $F$. alocis may be modulating TLR2 signaling to block or induce neutrophil effector functions. Moreover, this is the first study to assess the functional mechanisms of murine neutrophils against $F$. alocis, which will be useful in the context of animal models.

In addition to species differences, one of the concerns of these experiments was that since the mouse neutrophils were isolated from the bone marrow, they would be less responsive than human cells, which are fully differentiated when isolated from peripheral blood. However, the responses of wild-type bone marrow neutrophils (BMN) showed similar trends as their human cell counterpart. When challenged with $F$. alocis, both mouse and human neutrophils phagocytize $F$. alocis efficiently but are unable to eliminate the intracellular oral pathogen. This is because $F$. alocis blocks neutrophils' phagosome maturation in both species by inducing minimal ROS generation and inhibiting the fusion of azurophilic granules. Therefore, mouse neutrophils provided a suitable model to study F. alocis challenge, especially neutrophils with genetic modifications. Early signaling through TLR2 is associated with neutrophil migration, phagocytosis, cytokine production, and direct microbicidal functions like the generation of reactive oxygen species, and granule exocytosis $[327,358]$. Previous work from our laboratory showed that TLR2 ligation by $F$. alocis has a direct impact on neutrophil migration and granule exocytosis [275], but it was unknown if TLR2 played a role in phagocytosis or killing of $F$. alocis. 
Although TLR2 is not a phagocytic receptor, ligation of TLR2 will activate signaling pathways that enhance the efficiency of phagocytosis and inflammatory responses in macrophages $[359,360]$. However, in our experimental system, TLR2 did not have an effect on phagocytosis of $F$. alocis. A caveat to this finding is the fact that phagocytosis was synchronized to increase the interaction between cells and used complement opsonized bacteria, which already initiates an efficient mechanism of uptake. I also did not observe a difference in the ROS generation between WT and TLR2 $2^{-/}$ neutrophils, which is consistent with a study that used human neutrophils, TLR2 blocking antibodies and Paracoccidioides brasiliensis [361].

Studies of mice deficient in either TLR2 or MyD88 have found that loss of either of these proteins results in impaired ability to clear several types of bacteria, fungi and parasites from infected macrophages or mice [362-368]. This defect was attributed to decreased phagocytosis, defective phagosome maturation, or decreased oxidative burst, suggesting that both TLR2 and MyD88 are indispensable in innate immune defense against pathogens. However, when TLR2 is absent, neutrophils lose their ability to generate cytokines, but can kill and degrade viable $F$. alocis more efficiently due to a partial restoration of myeloperoxidase recruitment to the phagosome. Also, MyD88 did not affect phagocytosis, killing, ROS generation or degradation at early timepoints.

I speculate that $F$. alocis may be modulating the TLR2 pathway to block antimicrobial mechanisms, but when TLR2 is not available, a redundant pathway is activated to enhance elimination of $F$. alocis. It has previously been shown that TLR2 and TLR9 can synergize to enhance immune responses [369, 370], but it has not been assessed whether TLR9 is involved in F. alocis-induced signaling. TLR9 can be activated by 
unmethylated CpG containing DNA from bacteria or mitochondria [371], so while $F$. alocis may not be recognized by its DNA after lysis by the neutrophil phagosome, TLR9 could still be activated. Notably, TLR9 signaling is MyD88-dependent but IRAK-4 independent [372], hinting at a possible role in the neutrophil response against $F$. alocis. Surprisingly, the phosphorylation of IRAK4 was absent in F. alocis challenge but appeared normally after FSL-1 stimulation. While more experiments are needed to determine if this response has biological significance in the context of periodontitis and is actively caused by the viable bacterium, defective IRAK4 signaling has substantial implications for disease progression in other pathologies. For example, the kinase activity of IRAK4 is a prerequisite for mounting innate immune responses against intracellular bacteria like Listeria monocytogenes, Pseudomonas aeruginosa, S. aureus or Mycobacterium smegmatis $[373,374]$. IRAK4 and TLR9 have also both been linked to neutrophil granule movement $[375,376]$.

Alternatively, $F$. alocis could be inducing crosstalk between TLR2 and another receptor or signaling pathway that is disrupted when TLR2 is not present. F. alocis and other oral pathogens are notorious for disrupting the complement system $[64,377] . P$. gingivalis, evades clearance by neutrophils and promotes a pro-inflammatory environment by stimulating cross-talk between TLR2/1 and C5aR that results in the degradation of MyD88 [158, 244]. This reroutes signaling through another adaptor protein, MyD88 adaptor-like (Mal, also known as TIRAP), which activates the PI3K pathway instead of the MAPK pathway and increases the release of pro-inflammatory cytokines while decreasing the efficiency of neutrophil microbicidal mechanisms. The TLR2-PI3K signaling resulted in the bacterial phagosome escaping lysosomal degradation, so ultimately, this results in 
impaired clearance of systemic bacteria. However, when I tested this possibility in our system, $F$. alocis challenge did not cause MyD88 to be degraded, and cytokine production was completely dependent on MyD88 expression, indicating that the mechanism of pathogenicity is not the same between both of these oral pathogens. Nonetheless, at this time I cannot rule out the possibility that PI3K activation or complement receptor cross talk is happening during $F$. alocis infection.

Similarly, complex mechanisms regulate signaling by TLR2 from multiple cellular locations. TLR2 and its heterodimer partners, TLR1 and -6 , can be found on phagosomes containing zymosan particles, Chlamydia, and Borrelia species [378-381]. TLR2 heterodimers employ several accessory molecules like mannose binding lectin (MBL) and CD36, that can synergize with TLR2/6 to recognize new ligands and drastically increase the inflammatory responses downstream of TLR2 ligation [379, 382-384]. Through the actions of these accessory molecules, TLR $2 / 6$ will be internalized in the process of phagocytosis and can continue to signal from the phagosome, especially to enhance the production of cytokines and phagosome maturation in macrophages [366, 378, 385]. Although they could be playing a role in F. alocis modulation of signaling, I have not assessed either one of these accessory proteins.

Overall, I demonstrate that like other oral pathogens, F. alocis is blocking microbicidal mechanisms in neutrophils while promoting inflammation through the release pro-inflammatory cytokines; a phenotype that hinges on signaling from TLR2 and MyD88. As the first study to delineate the $F$. alocis strategies to control neutrophil effector functions by interrupting intracellular signaling and demonstrates that this topic is a rich source of 
knowledge that will enhance our understanding of how oral bacteria undermine the innate immune system. 
A.

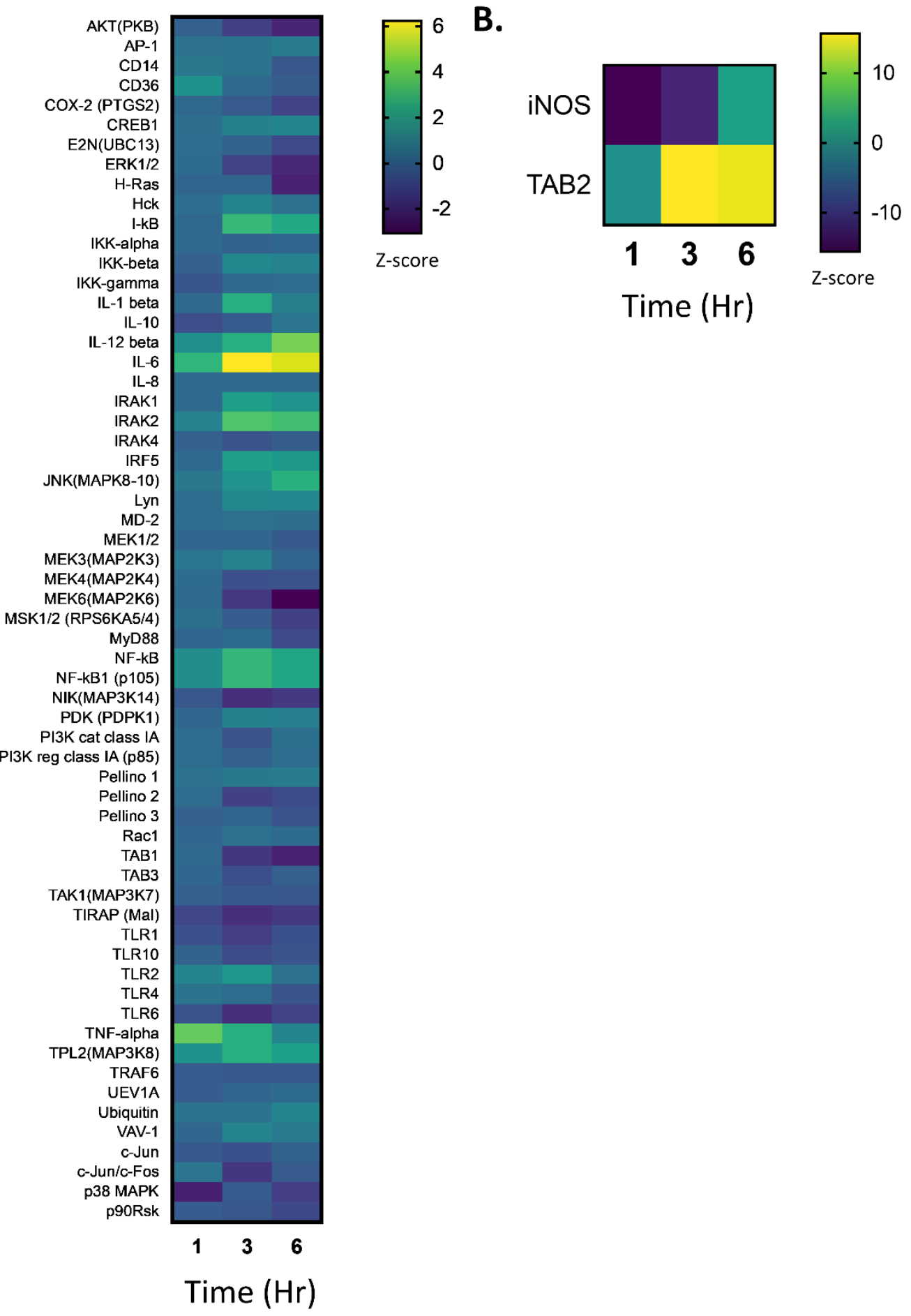

Figure 3.2: $F$. alocis induces changes in gene expression of genes in TLR signaling pathway. 
Figure 3.2: (A) Heatmap shows the differential expression of genes in the TLR2 Signaling Pathway from MetaCore. Gene expression data was generated from Illumina RNA sequencing performed on human neutrophils from 4 individual healthy donors that were either left unstimulated or challenged with $F$. alocis for 1, 3 or 6 hours. (B) From the list of genes from A, the two genes plotted had extremely large changes in gene expression and are plotted separately. 
A.

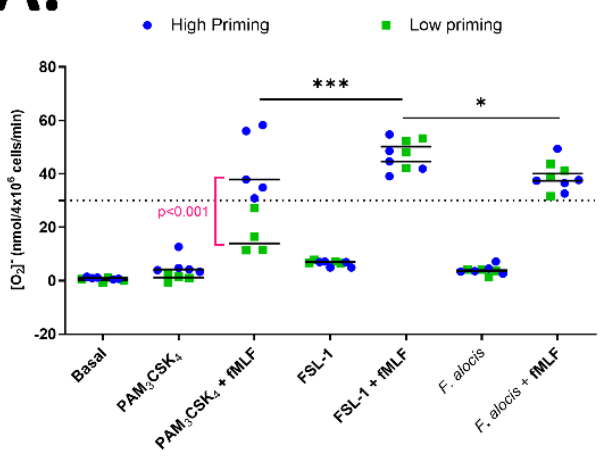

B.

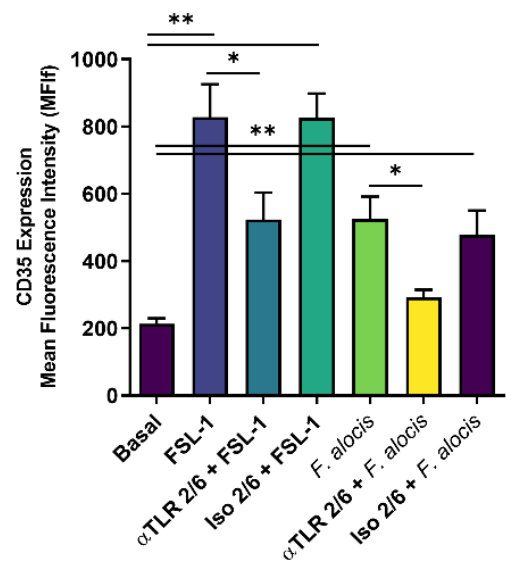

C.

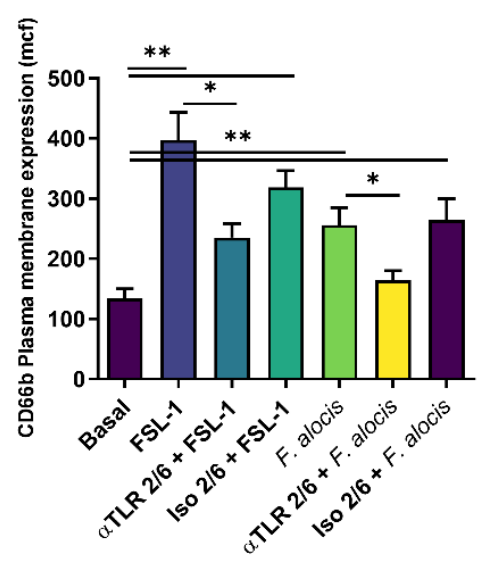

FIGURE 3.3: $F$. alocis is recognized by TLR2/6 heterodimers. 
FIGURE 3.3: (A) Human neutrophils were incubated with buffer (basal), PAM3CSK4 (TLR2/1 agonist), FSL-1 (TLR2/6 agonist), or $F$. alocis (MOI 10:1) or followed by fMLF stimulation. Superoxide release was measured by spectrophotometer as the colorimetric reduction of ferricytochrome C. Dotted line indicates the cut-off used for high and low priming donors. Data shows the mean \pm superoxide release from 7 total high and low priming donors. A two-way ANOVA with Bonferroni post hoc tests was performed. Human neutrophil were untreated or pre-treated with TLR2/6 blocking antibodies or isotype controls before stimulation with FSL-1 for 30 min or F. alocis for 60 min. Then, cells were analyzed by flow cytometry for expression of (B) secretory vesicles (CD35) or (C) specific granules (CD66b) as a measure of granule mobilization. Data are plotted as the average mean channel fluorescence from 4-7 independent experiments. A one-way ANOVA with Tukey post-hoc tests was performed on graphs B and C. * $=\mathrm{p}>0.05, * *=\mathrm{p}$ $>0.01, * * *=\mathrm{p}>0.001$ 

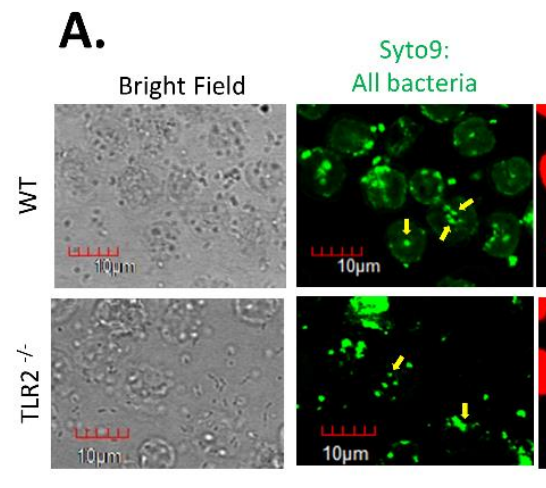

Propidium lodide:

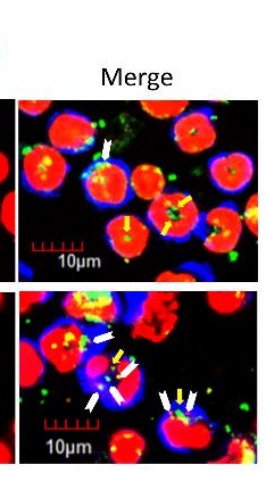

B. Dead bacteria

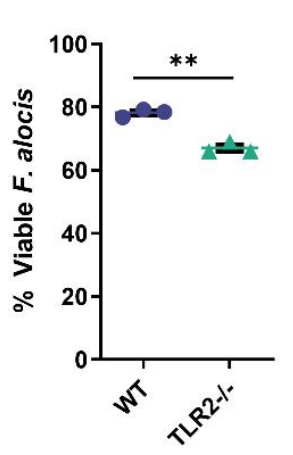

C.

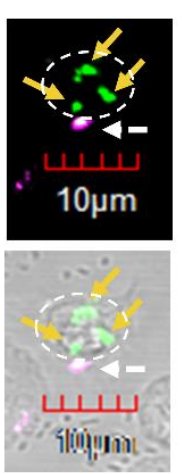

D.

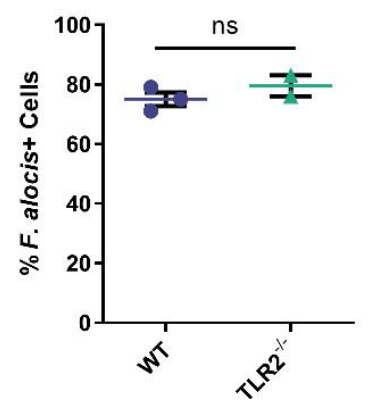

E.

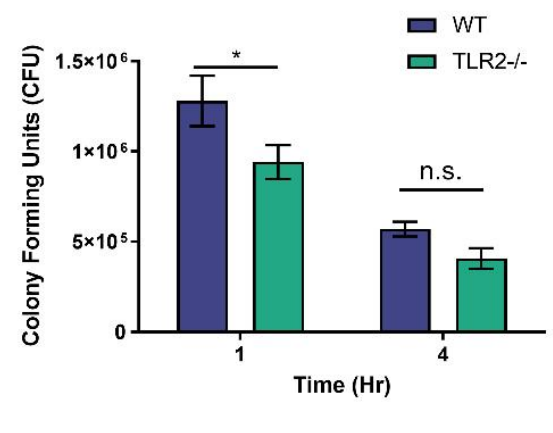

FIGURE 3.4: F. alocis survival is partially linked to TLR2 signaling. 
FIGURE 3.4: Bone marrow neutrophils $(\mathrm{BMN})$ were isolated from WT or TLR2 ${ }^{-/}$mice and challenged with $F$. alocis (MOI 10) for 1 hour followed by staining with BacLight dyes, Syto9 and Propidium Iodide (PI), to determine bacterial viability. (A) Representative images show the BMN cortical actin (Blue), the syto9-labeled F. alocis (green), and PIlabeled non-viable bacteria (red/yellow/orange). Yellow arrows point to intracellular viable F. alocis while white arrowheads point to intracellular, non-viable $F$. alocis. (B) The viability of intracellular F. alocis was assessed in Z-stacks from 100 infected cells. Data are plotted as the mean \pm SEM of the $\%$ viable $F$. alocis from 3 mice each over two independent experiments. (C) BMN from WT or TLR2 ${ }^{-/-}$mice were challenged with viable F. alocis (MOI 10) for 1 hour. Following incubation, cells were fixed and differentially stained for extracellular (purple or white bacteria, white arrow) or intracellular (green bacteria, yellow arrow) bacteria using anti-F. alocis antibodies. (D) 100 neutrophils from 2-3 mice in a single independent experiment were assessed for phagocytosis of $F$. alocis and graphed as the $\%$ F. alocis+ cells \pm SEM. (E) BMN from WT or TLR2 ${ }^{-1-}$ mice were challenged with $F$. alocis (MOI 10) for 1 and 4 hours followed. The total pellets of this interaction were plated in duplicates and grown anaerobically for 72 hours. Data are shown as the average \pm SEM of colony forming units from 3 mice completed over 2 independent experiments. An unpaired student's T test was used to determine statistical significance in B and D. A two-way ANOVA with Bonferroni post-hoc tests were used to determine the significance of E. $*=p>0.05, * *=p>0.01, * * *=p>0.001$. 

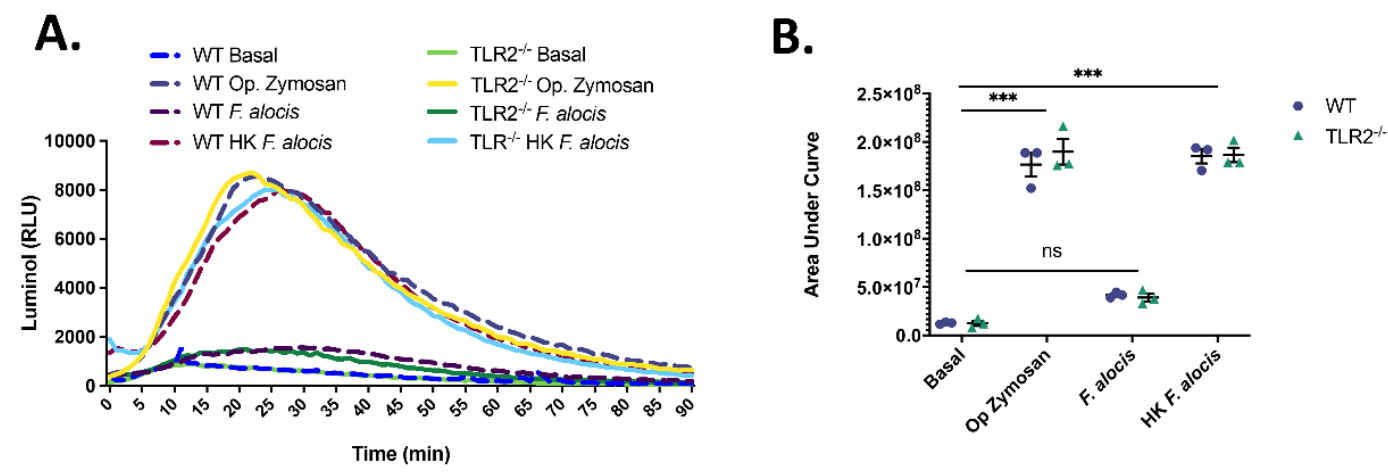

\section{FIGURE 3.5: $F$. alocis induces minimal ROS generation from both WT and TLR2 ${ }^{-/-}$}

bone marrow neutrophils. BMN from WT and TLR2 $2^{--}$mice were left unstimulated (basal) or stimulated with opsonized zymosan or F. alocis in the presence of luminol, and the light output was measured each minute during a 90-minute kinetic experiment. (A) Shows a representative kinetic output curve for one experiment. (B) Shows the area under the curve from 3 independent experiments where each dot is a mouse. A two-way ANOVA with Bonferroni post-hoc tests was used to determine the statistical significance of B. No differences were detected between mouse strains, so the lines indicate significance between the conditions for both mice. $*=p>0.05,{ }^{* *}=p>0.01,{ }^{* * *}=p>0.001$ 
A.

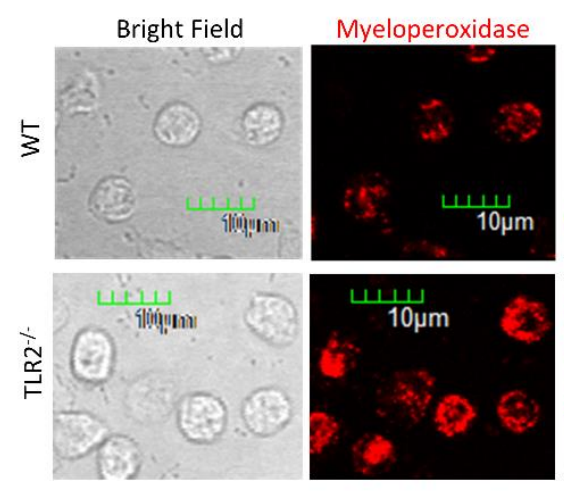

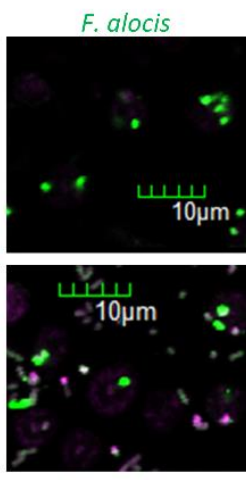

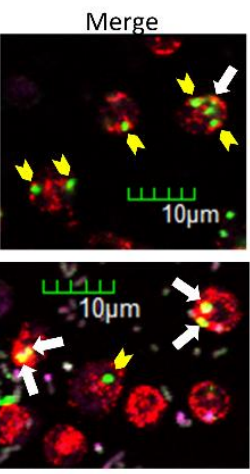

B.

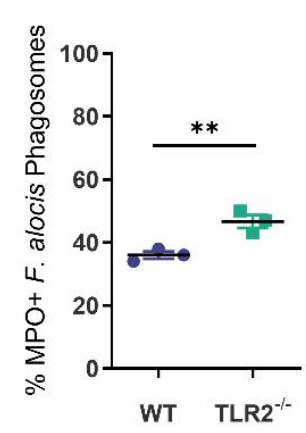

D.

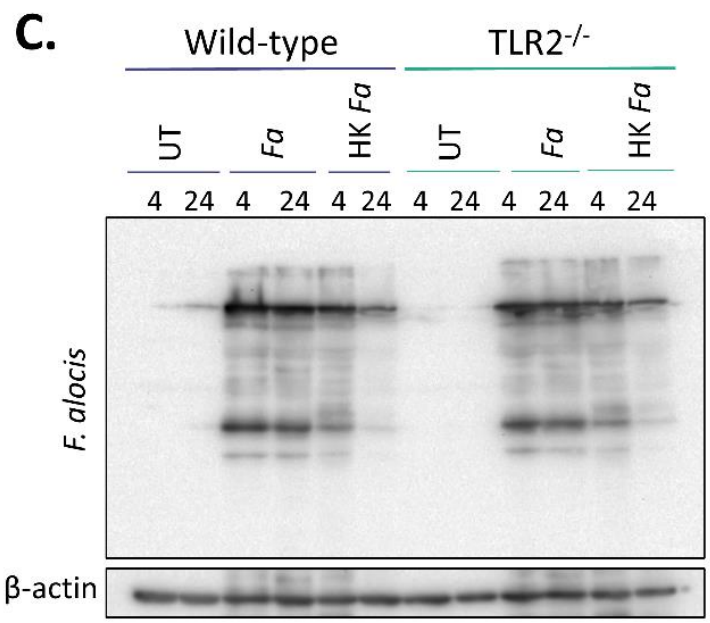

$\begin{array}{ll}\square \text { WT UT } & \square \text { TLR2-/-UT } \\ \square \text { WTFa } & \square \text { TLR2-/-Fa } \\ \square \text { WT HK } & \square \text { TLR2--HK }\end{array}$

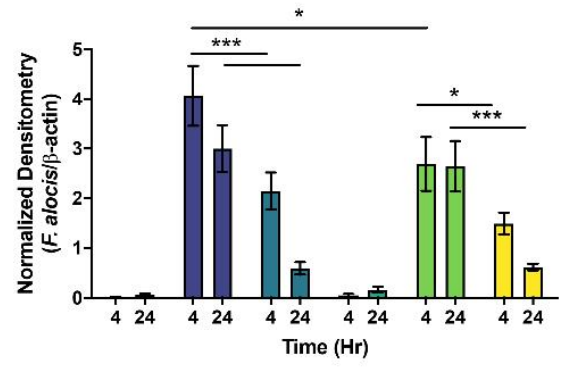

FIGURE 3.6: F. alocis modulates TLR2 signaling to block phagosome maturation. 
FIGURE 3.6: (A) BMN from $\mathrm{WT}$ and $\mathrm{TLR} 2^{-/-}$mice were challenged with $F$. alocis for 1 hour. Staining shows myeloperoxidase (MPO, azurophilic granules, red), extracellular $F$. alocis (purple/white), and intracellular F. alocis (green). The phagosomes of 100 infected neutrophils from 3 mice of each genotype were assessed for $50 \%$ co-localization of intracellular F. alocis and myeloperoxidase. Yellow arrows show MPO negative phagosomes while the white arrows indicate MPO positive phagosomes. (B) Data show the average $\%$ of $F$. alocis phagosomes that co-localized with myeloperoxidase \pm SEM from 3 mice in one independent experiment. An unpaired student's T test was used to determine statistical significance. (C) The representative western blot shows the whole cell lysates from WT and TLR2 ${ }^{-/-}$BMN that were left untreated (UT), challenged with $F$. alocis $(F a)$, or heat-killed $\mathrm{F}$. alocis (HK $F a$ ) for 4 or 24 hours and probed for $F$. alocis and $\beta$ actin. (D) Shows the mean normalized densitometry \pm SEM of $F$. alocis proteins from * mice completed over 3 independent experiments. A two-way ANOVA with Bonferroni post-hoc tests was used to determine the statistical significance. $*=p>0.05, * *=p>0.01, * * *=p$ $>0.001$ 


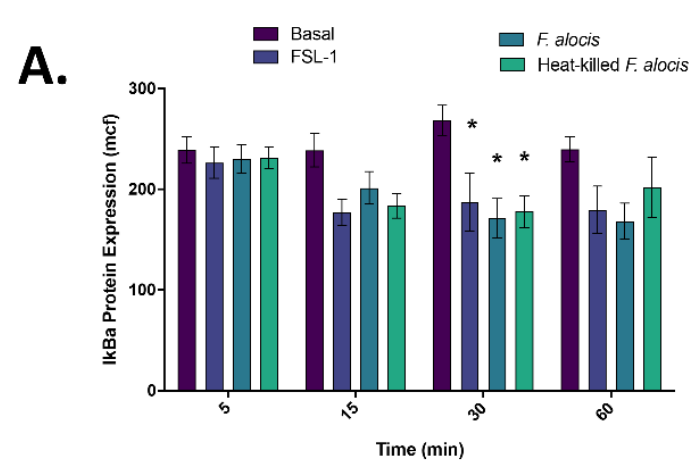

B.
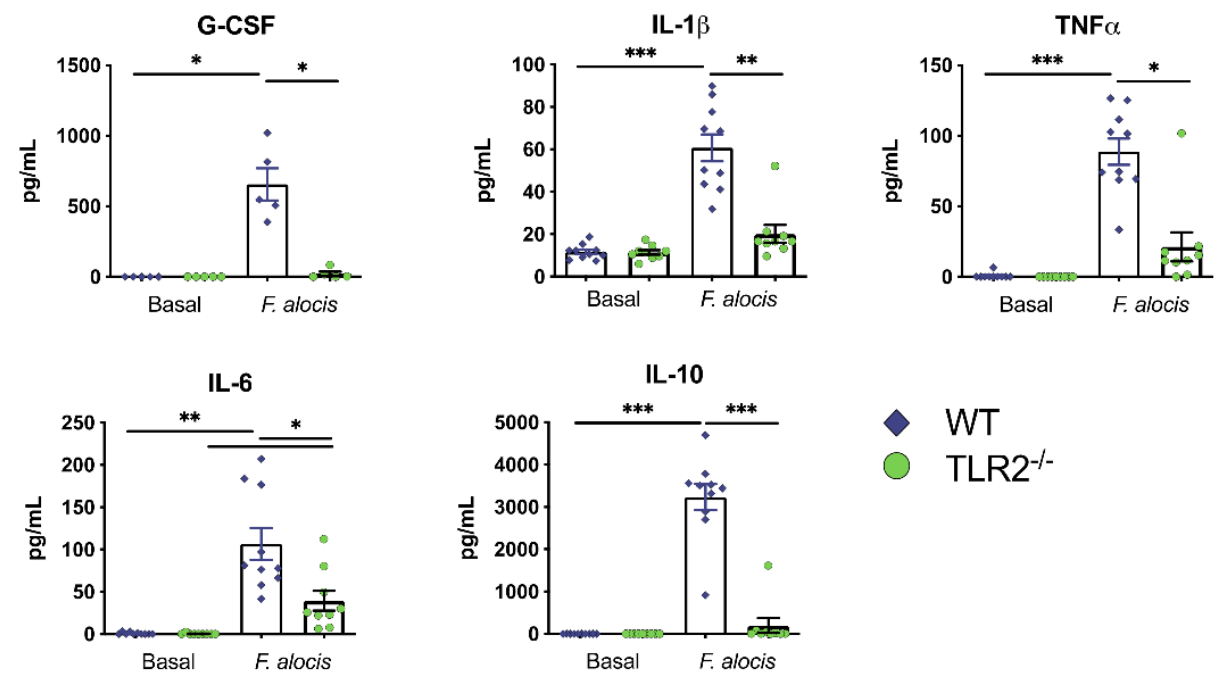

- TLR2-/-

C.
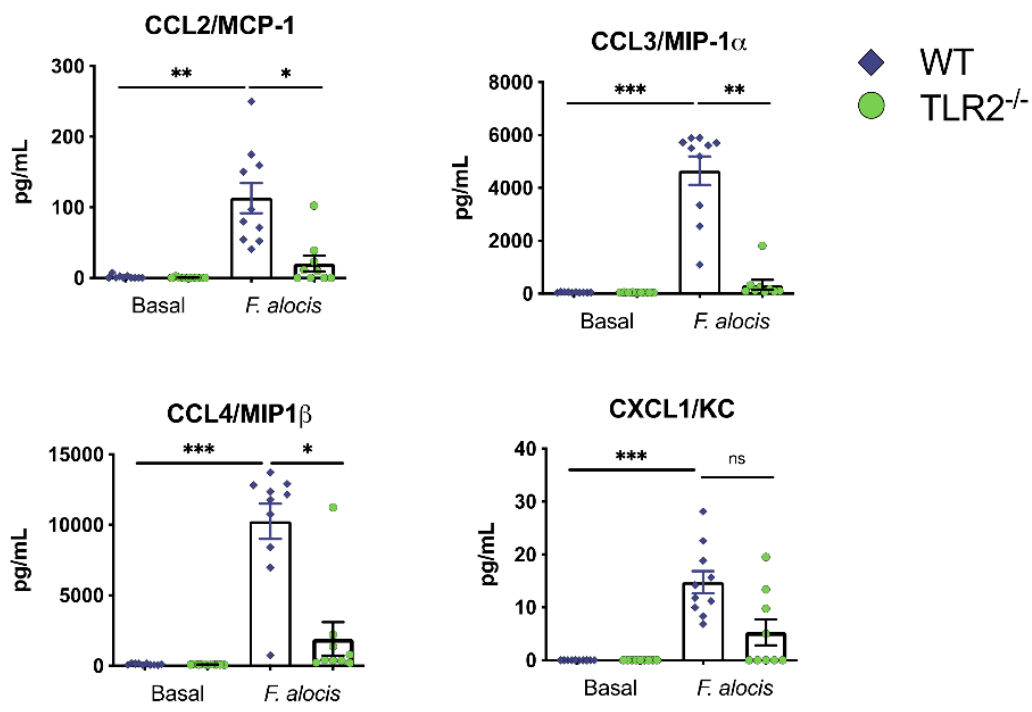

FIGURE 3.7: $F$. alocis activates NF-אB and induces the production of cytokines through TLR2. 
FIGURE 3.7: (A) Activation of NF-אB was tested indirectly by intracellular staining of human neutrophils for $\mathrm{IkB} \alpha$ after no stimulation (untreated) or stimulation with LPS, or $F$. alocis (MOI 10). Data shows the mean channel fluorescence (MCF) \pm SEM of I $\kappa \mathrm{B} \alpha$ in samples from 4 independent experiments. Asterisks denote significance compared to basal levels of IאB $\alpha$. WT and TLR2 ${ }^{-/-}$BMN were untreated or challenged with F. alocis for 24 hours. Supernatants were collected and tested for the presence of cytokines (B) and chemokines (C) using a multiplex assay. Data shows the cytokine \& chemokine production by neutrophils from 5-10 animals, conducted over 3-4 experiments. Error bars show SEM. Two-way ANOVAs with Bonferroni post-hoc tests were completed on all plots to determine statistical significance. $* \mathrm{P}<0.05 ; * * \mathrm{P}<0.01$; *** $\mathrm{P}<0.001$. 
A

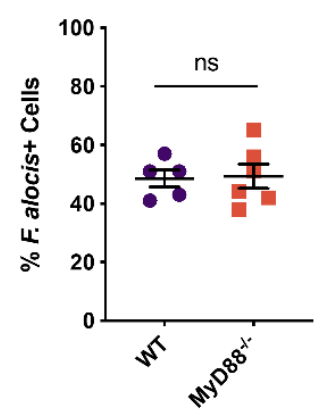

B.
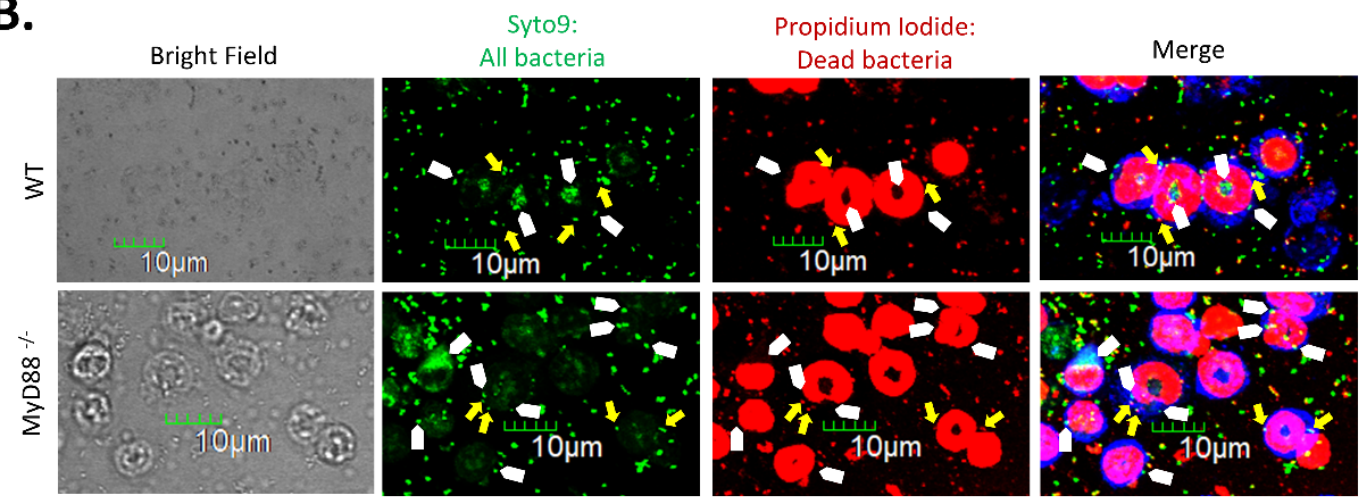

C.

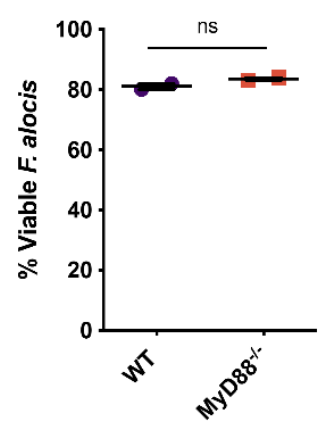

D.

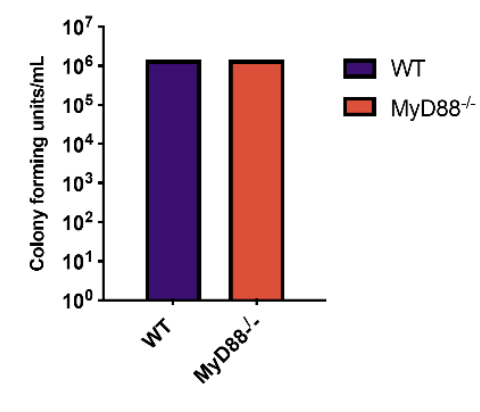

FIGURE 3.8: MyD88 signaling is not required for $F$. alocis viability. 


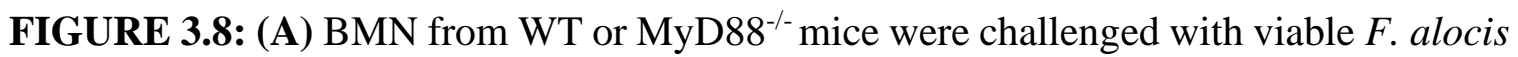
(MOI 10) for 1 hour. Following incubation, cells were fixed and differentially stained for extracellular or intracellular bacteria using anti-F. alocis antibodies. 100 neutrophils were assessed for phagocytosis of $F$. alocis over the course of 3 independent experiments. Data are graphed as the $\%$ F. alocis+ cells \pm SEM from 5-6 mice. (B) Representative images show BMN from WT or MyD88 ${ }^{-/-}$mice that were challenged with F. alocis (MOI 10) for 1 hour and then the viability of $F$. alocis was verified as described in Figure 4.4A. Yellow arrows point to intracellular viable $F$. alocis while white arrowheads point to intracellular, non-viable $F$. alocis. (C) The viability of intracellular $F$. alocis was quantified from Zstacks of 100 infected cells. Data are plotted as the mean \pm SEM of the $\%$ viable $F$. alocis from 2 mice in one independent experiment. (D) CFU were plated from an inoculum of $F$.

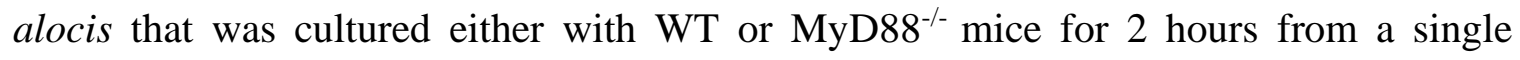
experiment with one mouse per genotype. An unpaired student's T test was used to determine statistical significance in $\mathrm{A}$ and $\mathrm{C}$. 
A.

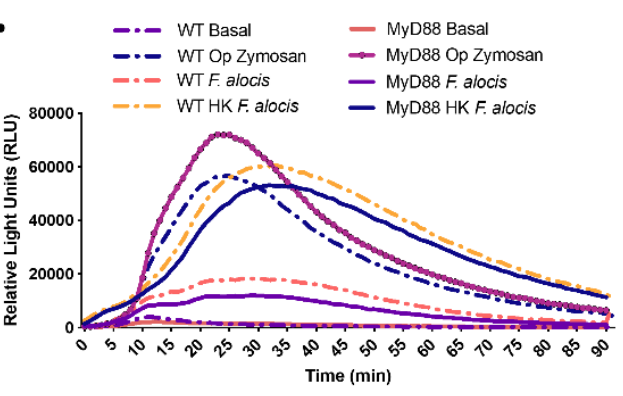

C.

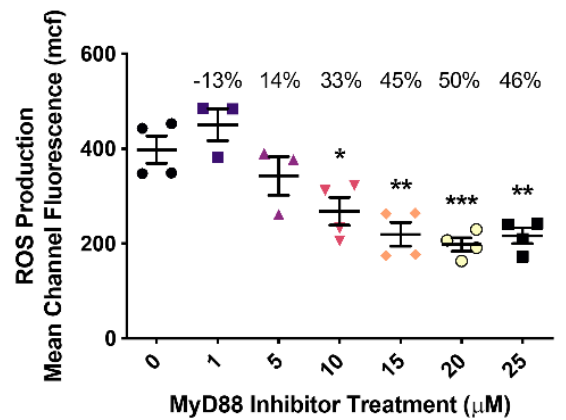

B.

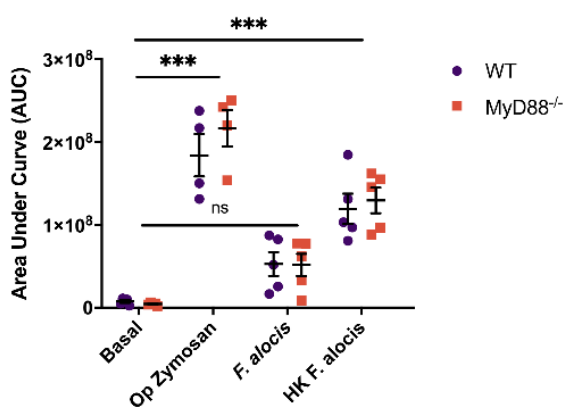

D.

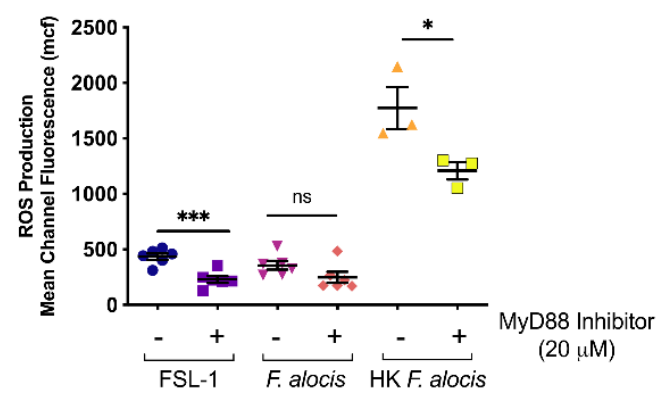

FIGURE 3.9: $F$. alocis induces minimal ROS generation from both WT and MyD881- bone marrow neutrophils. 
FIGURE 3.9: $\mathrm{BMN}$ from $\mathrm{WT}$ and $\mathrm{MyD}^{-/ /}$mice were left unstimulated (basal) or stimulated with opsonized zymosan or F. alocis in the presence of luminol, and the light output was measured each minute during a 90-minute kinetic experiment. (A) Shows a representative kinetic output curve for one experiment. (B) Shows the area under the curve from 4-5 independent experiments where each dot is a mouse. A two-way ANOVA with Bonferroni post-hoc tests was used to determine the statistical significance of $\mathrm{B}$. No differences were detected between mouse strains, so the lines indicate significance between the conditions for both mice. (C) Human neutrophils were pre-treated with increasing concentrations of ST-2825, a MyD88 inhibitor for 60 minutes, followed by stimulation with FSL-1 for * minutes. The decrease in ROS production was measured via flow cytometer. Data are plotted as the average mean channel fluorescence \pm SEM from 3-4 independent experiments. Numbers above each condition indicate the average inhibition by the concentration shown. A one-way ANOVA with Tukey post-hoc tests was used to determine the statistical significance of C. (D) Using $20 \mu \mathrm{M}$ of the MyD88 inhibitor as pretreatment, human neutrophils were stimulated with viable or heat-killed $F$. alocis and tested for ROS generation. Data are plotted as the average mean channel fluorescence \pm SEM from 3-5 independent experiments. An unpaired student's T test was used to determine statistical significance between the pairs of conditions. $*=p>0.05, * *=p>0.01, * * *=p$ $>0.001$ 
A. Bright Field
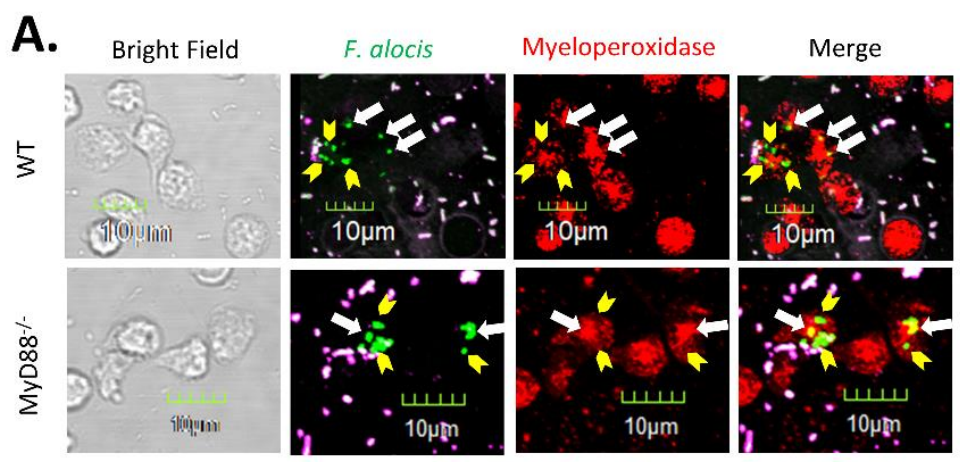

B.

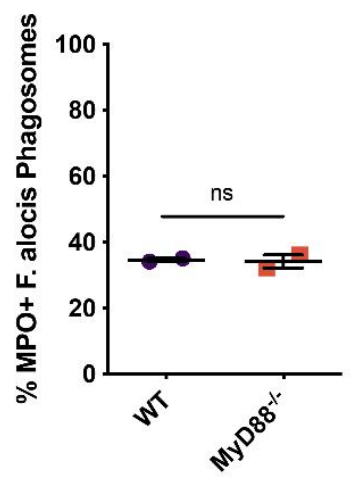

C.

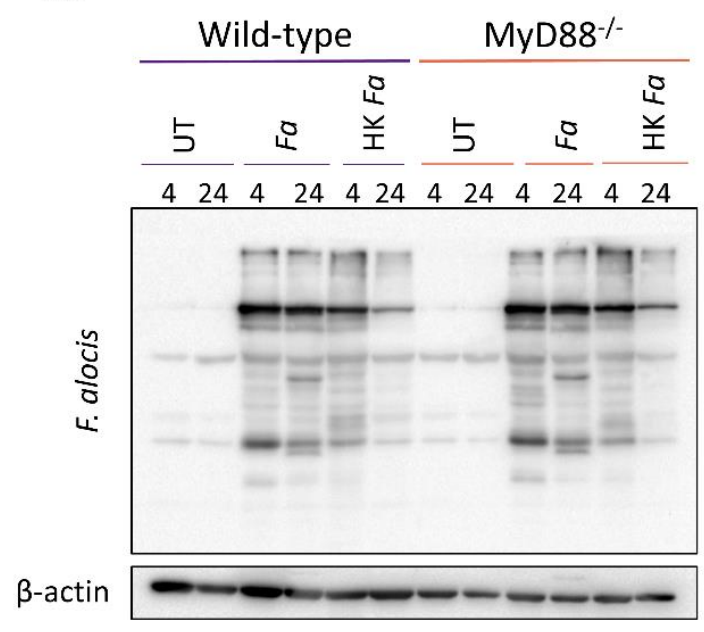

D.

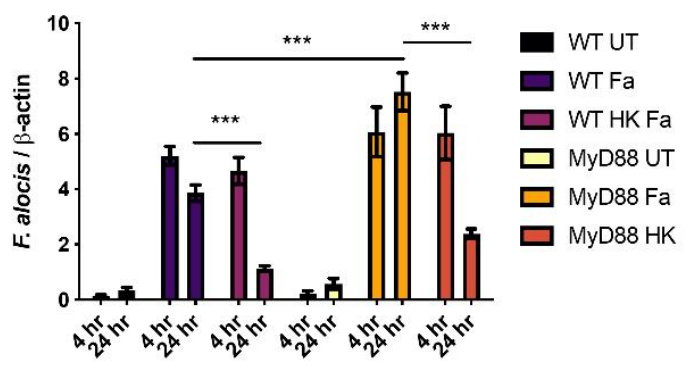

FIGURE 3.10: $F$. alocis phagosome maturation requires MyD88 at long timepoints. 
FIGURE 3.10: (A) BMN from WT and MyD88 $8^{-/-}$mice were challenged with $F$. alocis for 1 hour. Staining shows myeloperoxidase (MPO, azurophilic granules, red), extracellular $F$. alocis (purple/white), and intracellular F. alocis (green). Yellow arrows show MPO negative phagosomes while the white arrows indicate MPO positive phagosomes. (B) The phagosomes of 100 infected neutrophils from 2 mice of each genotype were assessed for $50 \%$ co-localization of intracellular $F$. alocis and myeloperoxidase. Data show the average $\%$ of $F$. alocis phagosomes that co-localized with myeloperoxidase \pm SEM from 2 mice in one independent experiment. An unpaired student's T test was used to determine statistical significance. (C) The representative western blot shows the whole cell lysates from WT and $\mathrm{MyD} 88^{-/-}$BMN that were left untreated (UT), challenged with $F$. alocis $(\mathrm{Fa}$ ), or heatkilled F. alocis (HK Fa) for 4 or 24 hours and probed for $F$. alocis and $\beta$ actin. (D) Shows the mean normalized densitometry \pm SEM of $F$. alocis proteins from 5 mice for each genotype completed over 2 independent experiments. A two-way ANOVA with Bonferroni post-hoc tests was used to determine the statistical significance. $*=p>0.05$, $* *=\mathrm{p}>0.01, * * *=\mathrm{p}>0.001$ 
- WT O MyD88 ${ }^{-1-}$
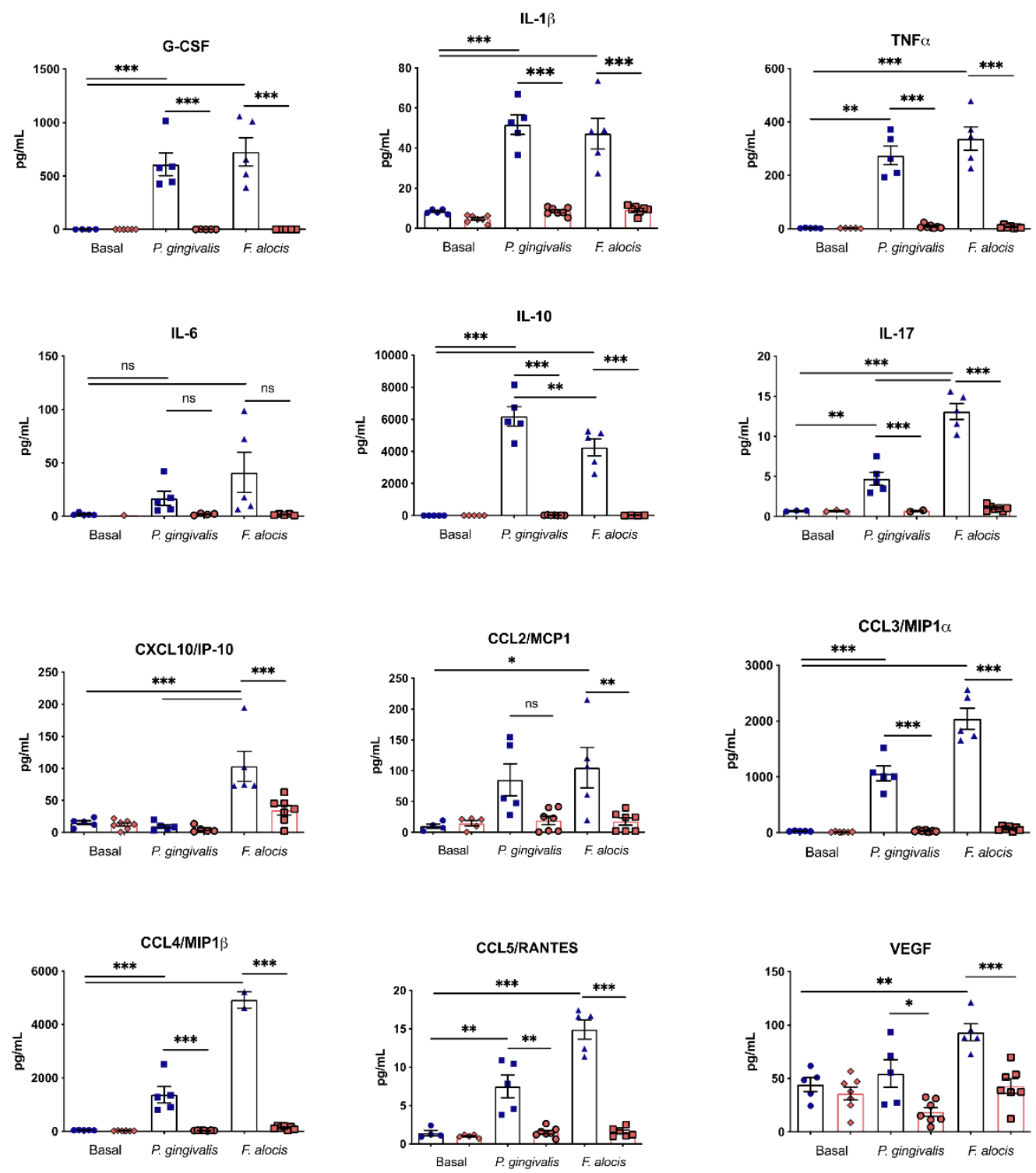

FIGURE 3.11: $F$. alocis-induced cytokine production is MyD88-dependent. WT and MyD88 $^{-/-}$BMN were untreated or challenged with P. gingivalis or F. alocis for 24 hours. Supernatants were tested for cytokines and chemokines using a multiplex assay. Data shows cytokine \& chemokine release from 5 mice, conducted over 2 experiments. Error bars show SEM. Two-way ANOVAs with Bonferroni post-hoc tests were completed on all plots to determine statistical significance. $* \mathrm{P}<0.05 ; * * \mathrm{P}<0.01 ; * * * \mathrm{P}<0.001$. 
A.

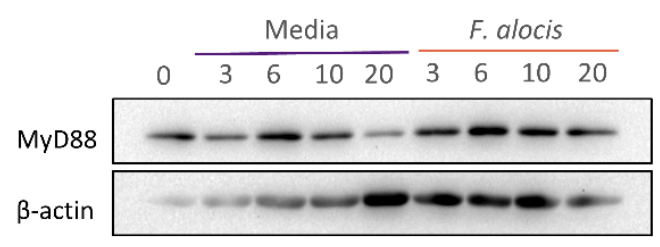

B.

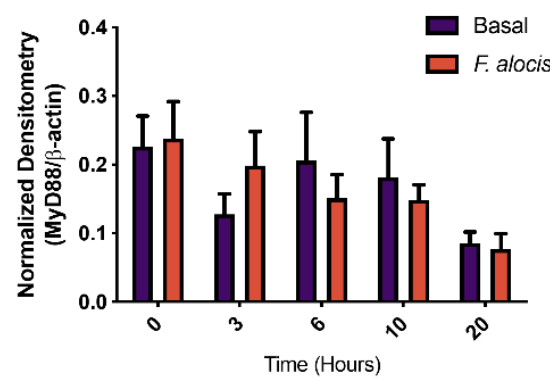

C.

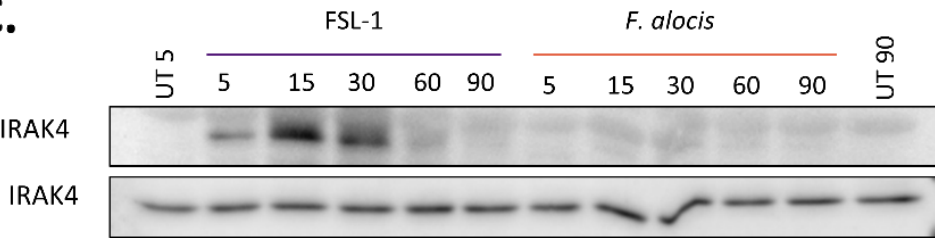

D.

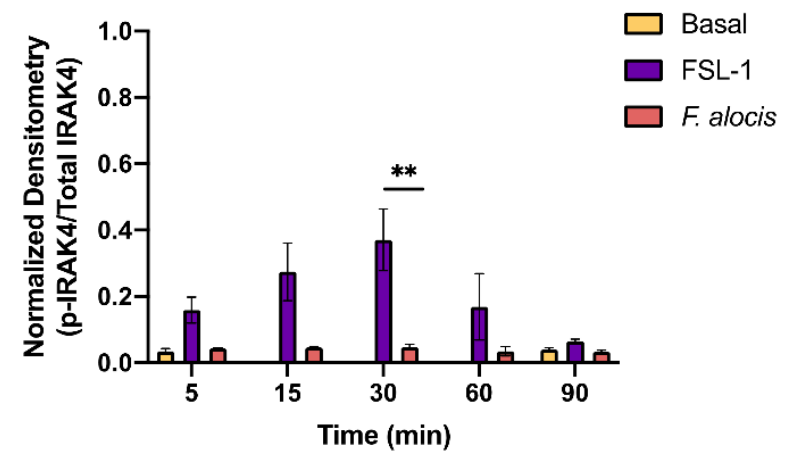

FIGURE 3.12: $F$. alocis does not affect MyD88 protein levels and does not activate IRAK4. 
FIGURE 3.12: (A) Western blot showing MyD88 expression in human neutrophils cultured in media or stimulated with $F$. alocis for 3, 6, 10 or 20 hours. (B) Pooled normalized densitometry from 4 independent experiments was plotted \pm SEM. A two-way ANOVA with Bonferroni post-hoc tests determined statistical significance. (C) Phosphorylation of IRAK4 in whole cell lysates from human neutrophils cultured in media or stimulated with $F$. alocis for 3, 6, 10 or 20 hours. (D) Normalized densitometry from 2 independent experiments was pooled and plotted \pm SEM. A two-way ANOVA with Bonferroni post-hoc tests determined statistical significance for B and D. * $=p>0.05$, $* *=\mathrm{p}>0.01, * * *=\mathrm{p}>0.001$ 


\section{CHAPTER 4:}

'TIL DEATH DO US PART: $F$. ALOCIS EFFECT ON NEUTROPHIL LIFESPAN.

\section{Introduction:}

Neutrophils are generated in the bone marrow at a rate of $10^{11}$ cells/day, and in the bloodstream they are the most abundant leukocyte in circulation [386, 387]. To maintain homeostasis, this large-scale production must be counteracted by efficient removal since dysregulated neutrophil presence leads to profound and devastating conditions ranging from neurologic defects to increased susceptibility to infections $[76,93,388]$. One of the most successful mechanisms for the normal turnover of neutrophils is programmed cell death, or apoptosis, which occurs in circulating neutrophils after 24 hours [389]. Apoptosis is a non-inflammatory process, and in neutrophils it is characterized by membrane blebbing, cell body shrinkage, cytoplasmic vacuolation, condensation of DNA, and changes in the nuclear morphology from multi-lobulated to a round, dense shape [389391]. Neutrophils that have become apoptotic can be detected by using end-point measurements like changes in nuclear morphology, DNA fragmentation, and accumulation of phosphatidylserine (PS) in the outer membrane leaflet.

The molecular regulation of neutrophil apoptosis is a sophisticated process, but generally, whether a neutrophil lives or dies is determined by the ratio of pro-survival or 
pro-apoptotic regulatory factors [392]. This ratio can change when apoptosis is activated through one of three main pathways: intrinsic, extrinsic, or phagocytosis-induced cell death (PICD). Both the intrinsic and extrinsic apoptosis pathways are mediated through the actions of a family of cysteine proteases called caspases. The initiator caspases, caspase 8 (extrinsic pathway) and caspase 9 (intrinsic pathway), both converge at the activation of caspase 3, which is called the executioner caspase. Caspase 3 activation is essential for programmed cell death because its activation is required to initiate the defining biochemical and biophysical changes that occur during apoptosis. In healthy cells, caspases are in the cytosol as pro-enzymes that further stay in their inactive state through association with proteins of the inhibitor of apoptosis (IAP) family (such as XIAP, cIAP, cIAP2). For example, XIAP binds directly to caspases 9 and 3 and inhibits their processing and activation [393, 394]. Once the pro-caspases are cleaved into their active form, their protease activity will organize and set apoptosis processes in motion.

The intrinsic pathway is activated for both constitutive and stimulated apoptosis and is regulated at the level of the mitochondria. The key step in this pathway is the permeabilization of the mitochondrial outer membrane (MOMP) by pro-death proteins Bax and Bak, which oligomerize and are inserted into the membrane. This disrupts the organelle's membrane potential and causes cytochrome C, Smac/DIABLO, and Htra2/Omi to be released from the intermembrane space into the cell's cytosol [395-397]. The released cytochrome $\mathrm{C}$ will bind to apoptosis protease activating factor (APAF) to form a complex that recruits and cleaves inactive procaspase-9 into its active form. The other mitochondriaderived proteins will also augment the cell death signal by directly inactivating pro-survival factors [397]. Bax and Bak are pro-apoptotic members of the BCL-2 family, but within the 
same family of proteins are Myeloid Leukemia Cell Differentiation Protein (MCL)1 and BCL2-Related Protein A1 (A1, also known as Bfl-1), which have pro-survival effects. MCL-1 and A1 have short half-lives and require continuous synthesis to carry out their pro-survival effects, whereas Bax and Bak have long half-lives [398-400]. Consistent with this, the mRNA and protein expression of MCL-1 and A1 is high in healthy neutrophils to prevent the disruption of the mitochondrial membrane, but as neutrophils age, levels of MCL-1 and A1 decline while pro-death factors remain, initiating spontaneous apoptosis [401-404].

The extrinsic apoptotic pathway is activated by the ligation of surface death receptors that recognize Fas ligand, TRAIL, or TNF $\alpha$. Upon ligation, the receptors will oligomerize and form a complex that recruits and activates caspase 8 . In neutrophils the extrinsic pathway is not sufficient to induce cell death [405]. In fact, the signal has to also be amplified by the intrinsic pathway through Bid, a pro-apoptotic protein that links both pathways together. Caspase 8 activation by the extrinsic pathway will activate Bid, which will translocate to the mitochondria and activate Bax and Bak to initiate the intrinsic pathway [406]. Finally, literature suggests that the extrinsic pathway does not appear to play a role in the constitutive apoptosis pathways, but has a more important role in neutrophil apoptosis during infection and inflammation [405, 407-410].

The third pathway that induces apoptosis is phagocytosis-induced cell death (PICD), which was first described in 1992 [411]. While the mechanism is still poorly understood, PICD requires the production of ROS, and is independent of death receptor ligation [107, 412, 413]. Phagocytosis of bacterial pathogens, including Burkholderia cepacia, Borrelia hermsii, Staphylococcus aureus, Streptoccocus pyogenes, and Listeria 
monocytogenes, is followed by global changes in neutrophil gene expression [109]. Cells undergoing PICD have differentially affected pathways related to metabolism, cytokine production, receptors, signal transduction, transcription, and host defense. As a consequence of the changes in gene expression, cells undergoing PICD have a restricted proinflammatory capacity via a general impairment in chemotaxis, phagocytosis and degranulation [392]. Thus, in the context of infection, PICD represents a non-inflammatory mechanism to clear tissues of effete neutrophils that contain non-viable or partially degraded microbes.

When neutrophils migrate into sites of tissue inflammation, they display a prolonged lifespan and become resistant to FAS- and TNF- induced apoptosis, likely to promote a robust early response to infection [263]. This phenotype can be due to hypoxia in inflamed tissues [414, 415], or cues at the site of infection, since both host and microbederived factors can increase neutrophil lifespan. For example, the same cytokines (IL-1 $\beta$, TNF $\alpha$, GM-CSF, G-CSF, and IFN $\gamma$ ) that can recruit neutrophils to a site of infection will also delay their apoptosis [127, 416-419]. Similarly, the anaphylatoxin C5a, LPS, and lipoteichoic acid (LTA) are powerful chemotactic agents for neutrophils and protects them from apoptosis [416, 420]. In the context of infection, PICD is desirable for the resolution of inflammation, but many pathogens have evolved means to disrupt normal apoptotic processes to promote pathogenesis [421, 422]. Generally, intracellular pathogens like Anaplasma phagocytophilum, Chlamydia pneumoniae, and Francisella tularensis promote neutrophil viability to protect their replicative niche whereas extracellular microbes like $S$. pyogenes, S. aureus, and Pseudomonas aeruginosa accelerate apoptosis, trigger neutrophil lysis, or redirect cell death towards necrosis to evade intracellular killing [89]. All of these 
outcomes can delay resolution of inflammation because (1) prolonged neutrophil lifespan results in the accumulation of cells with increased pro-inflammatory potential, (2) accelerated neutrophil lifespan promotes bacterial colonization or dissemination, and (3) necrosis or lysis of neutrophils results in the release of toxic neutrophil components and other DAMPS that exacerbate inflammation. Therefore, precise regulation of neutrophil cell death is essential for curbing inflammatory responses.

In the context of periodontitis, not much is known about how periodontal pathogens affect neutrophil lifespan. Most of the published data on apoptosis of neutrophils with periodontal pathogens has been limited to very short timepoints (less than 3 hours), where the full extent of neutrophil survival cannot be gauged. Moreover, defects in apoptosis could be contributing to the progression of periodontitis, but this possibility has not been explored much.

In this chapter, I demonstrate that contact of neutrophils with $F$. alocis results in an upregulation of anti-apoptotic proteins, dampening of caspase activity, and delay of neutrophil apoptosis. The $F$. alocis cultured neutrophils retained their pro-inflammatory effector functions at longer timepoints as compared to untreated neutrophils.

\section{$\underline{\text { Materials \& Methods: }}$}

Neutrophil isolation: Human donor recruitment, blood draws, and materials required for this procedure were in accordance with the guidelines approved by the Institutional Review Board of the University of Louisville. Neutrophils were isolated from venous blood of healthy donors using plasma-Percoll gradients, as described previously [297]. 
Bacterial strains and growth conditions: F. alocis ATCC 38596 was cultured in brain heart infusion (BHI) broth supplemented $5 \mathrm{mg} / \mathrm{mL}$ yeast extract, L-cysteine $(0.05 \%)$ and arginine $(0.05 \%)$ for 7 days anaerobically at $37{ }^{\circ} \mathrm{C}$ as previously described $[275,276]$. Serum opsonization was performed by incubating $F$. alocis at $37^{\circ} \mathrm{C}$ for $20 \mathrm{~min}$ in $10 \%$ normal human serum (Complement Technology, Inc., Tyler, TX, USA). F. alocis was heatkilled by incubation at $90{ }^{\circ} \mathrm{C}$ for $60 \mathrm{~min}$. Non-viability was confirmed by incubation in culture media at same conditions used for the live organism. After opsonization, $F$. alocis was labeled with carboxyfluorescein succinimidyl ester (CFSE; $40 \mathrm{ng} / \mu \mathrm{l}$ ) for $30 \mathrm{~min}$ at room temperature in the dark and washed 3 times with PBS prior to use.

Cell culture and infection: Neutrophils $\left(4 \times 10^{6}\right.$ cells $\left./ \mathrm{mL}\right)$ were cultured at $37^{\circ} \mathrm{C}, 5 \% \mathrm{CO}_{2}$ in RPMI-1640 with L-glutamine or with cycloheximide (1 nM, Sigma), opsonized F. alocis (multiplicity of infection (MOI) 10, 50, 100), or opsonized heat-killed $F$. alocis (MOI 10). After 24 hours of culture, cells were split and processed as cytospins and stained with HEMA or stained for Annexin V/7-AAD testing using the commercially available APC Annexin V Apoptosis Detection Kit with 7-AAD (BioLegend, San Diego, CA, USA). Samples were read on a BD FACSCelesta flow cytometer and analyzed using the FlowJo software (Ashland, OR, USA). In experiments using neutralizing antibodies, neutrophils were pre-treated with TLR6 neutralizing antibody ( $50 \mathrm{ug} / \mathrm{mL}$, Invivogen) or isotype control (Rat IgG,50 ug/mL, Invivogen) for 60 minutes and/or TLR2 neutralizing antibody (50 $\mu \mathrm{g} / \mathrm{ml}$; clone TL2.1; BioLegend) or isotype control IgG2a kappa (50 $\mu \mathrm{g} / \mathrm{ml}$; clone MOPC173; BioLegend) for 30 minutes. 
Neutrophil Western Blotting: Neutrophils $\left(10 \times 10^{6}\right.$ cells $\left./ \mathrm{mL}\right)$ were cultured at $37^{\circ} \mathrm{C}$, $5 \% \mathrm{CO}_{2}$ in RPMI-1640 and left unstimulated, stimulated with soluble FasL $(500 \mathrm{ng} / \mathrm{mL}$, BioLegend), or challenged with live opsonized $F$. alocis for 3, 6, 12, or 24 hours. Then, cells were centrifuged at $6,000 \times g$ for $30 \mathrm{~s}$ and lysed for $30 \mathrm{~min}$ on ice in ice-cold lysis buffer $(20 \mathrm{mM}$ Tris- $\mathrm{HCl}$ [pH 7.5], $150 \mathrm{mM} \mathrm{NaCl}, 1 \%$ [vol/vol] Triton X-100, 0.5\% [vol/vol] Nonidet P-40, 20 mM NaF, 20 mM NaVO3, 1 mM EDTA, 1 mM EGTA, 5 mM phenylmethylsulfonyl fluoride [PMSF], $21 \mu \mathrm{g} / \mathrm{ml}$ aprotinin, $5 \mu \mathrm{g} / \mathrm{ml}$ leupeptin, and $4 \mathrm{mM}$ Diisopropyl fluorophosphates [DFP]). After protein estimation using the Pierce BCA protein assay kit (Thermo Scientific, Waltham, MA, USA), samples were adjusted to a concentration of $2 \mu \mathrm{g} / \mu \mathrm{L}$. Unless otherwise noted, $10-20 \mu \mathrm{g} / \mu \mathrm{L}$ of total cell lysates were separated by $12 \%$ SDS-PAGE and immunoblotted with antibodies for MCL-1 (1:500, Proteintech, Rosemont, IL), XIAP, Caspase 3 and $\beta$-actin (1:1000, Cell Signaling, Danvers, MA, USA. The appropriate secondary antibodies were used at 1:2000 dilution (Cell Signaling, Danvers, MA, USA). The ECL system (Amersham Pharmacia Biotech, Little Chalfont, United Kingdom) or the SuperSignal West Femto Maximum Sensitivity Substrate (Thermo Scientific,Waltham, MA, USA) was used to visualize antigen-antibody reactions. Densitometric values of each band were calculated using Image Lab software (BioRad, Hercules, CA, USA).

Luminescent Caspase Activity Assays: Neutrophils were left untreated or treated with staurosporine $(1 \mu \mathrm{M})$, Fas L (500 ng/mL), or opsonized F. alocis ( MOI 10:1) for 0, 3, 6 , 12,24 hours at $37{ }^{\circ} \mathrm{C}, 5 \% \mathrm{CO}_{2}$ in RPMI-1640. The activity of caspase $3 / 7,8$, and 9 was assayed using manufacturer's protocol from the Caspase-Glo assay kits from Promega 
(Madison, WI). Briefly, $100 \mu \mathrm{L}$ aliquots containing $5 \times 10^{4}$ neutrophils were transferred to white, flat bottomed 96-well plates in triplicates. Equal volume of the Caspase Glo-reagent was mixed into each well and left to incubate at room temperature for 45 minutes. Activity was assessed as by an end-point measurement of the luminescence generated from the cleavage of the Caspase-Glo reagent.

Fluorescence Caspase 3/7 Activity Assay: Neutrophils $\left(1 \times 10^{6}\right.$ cells in $\left.500 \mu \mathrm{L}\right)$ were left to adhere to serum-covered coverslips in 24 well plates for 30 minutes before they were untreated or stimulated opsonized F. alocis ( MOI 10:1). The infection was "synchronized" by a centrifugation step $\left(4 \mathrm{~min}, 600 \mathrm{~g}, 14^{\circ} \mathrm{C}\right)$ and the cells were cultured for $6,12,24$ hours at $37^{\circ} \mathrm{C}, 5 \% \mathrm{CO}_{2}$ in RPMI-1640. At the indicated timepoints, 1 drop of CellEvent ${ }^{\mathrm{TM}}$ Caspase-3/7 Green ReadyProbes ${ }^{\mathrm{TM}}$ Reagent (Invitrogen) was added per well (each containing $500 \mathrm{uL}$ ) and left to incubate at room temperature for 30 minutes. The wells were washed 3x with sterile PBS and the cells fixed with 4\% paraformaldehyde for $10 \mathrm{~min}$ at room temperature. After once more wash with PBS, the coverslips were mounted onto glass slides and imaged with a confocal microscope. 100 cells from throughout the coverslip were counted and evaluated for expression of GFP.

Assessment of DNA fragmentation: Neutrophils $\left(4 \times 10^{6}\right.$ cells $\left./ \mathrm{mL}\right)$ were stimulated with viable opsonized $F$. alocis (MOI 10:1) or were left untreated for 0, 3, 6, 12, 24, or 48 hours at $37{ }^{\circ} \mathrm{C}, 5 \% \mathrm{CO}_{2}$ in RPMI-1640. DNA fragmentation was determined using the ApoBRDU apoptosis detection kit (BD Biosciences), a modified TUNEL assay. Samples were labeled according to the manufacturer's instructions with minor modifications. PMNs were fixed 
with $4 \%$ paraformaldehyde for 60 min at $4{ }^{\circ} \mathrm{C}$, washed, and stained for 90 min. Samples were read on a BD FACSCelesta flow cytometer and analyzed using the FlowJo software (Ashland, OR, USA).

Transwell experiments: Neutrophils $\left(2 \times 10^{6}\right.$ cells in $600 \mu \mathrm{L}$ of RPMI) were added to the wells of a 24 well plate with transwells containing a $0.4 \mu \mathrm{M}$ filter in duplicates. In the top chamber, $100 \mu \mathrm{L}$ of either media or the opsonized $F$. alocis inoculum was added. A separate well received $F$. alocis into the well with the neutrophils for the "same" control. Then, the plate was cultured in an incubator at $37{ }^{\circ} \mathrm{C}, 5 \% \mathrm{CO}_{2}$. After 24 hours of culture, the duplicates were combined and stained for Annexin $\mathrm{V}$ and 7AAD as previously described.

Generation of Conditioned Media: Neutrophils from the percoll gradient separation were further enriched to obtain highly pure cells (>99\%) by negative magnetic selection using the Easy Eights EasySep Magnet and human neutrophil enrichment kit (Stemcell Technologies, Vancouver, BC, Canada), as previously described [298]. Cell purity was assessed by simultaneously staining with FITC-conjugated anti-CD66b (clone G10F5; BioLegend, San Diego, CA, USA) and APC-conjugated anti-CD16 (clone CB16; eBioscience, San Diego, CA, USA) antibodies and determining the percentage of CD66b+CD16+ cells using BD Celesta flow cytometer (BD Biosciences, San Jose, CA, USA). After purification, cells were resuspended the cells in clear RPMI medium supplemented with 5\% heat inactivated human serum (Atlanta Biologicals, Flowery Branch, GA, USA) for a concentration of $4 \times 10^{6} \mathrm{cells} / \mathrm{mL}$. Neutrophils were distributed 
into 12 well plates in triplicates. If needed, neutralizing antibodies were added for 30-60 minutes as listed above. Then, opsonized $F$. alocis (MOI 10) was added and phagocytosis synchronized (600g for $4 \mathrm{~min}$ at $\left.14^{\circ} \mathrm{C}\right)$. After incubation for 24 hours in $37^{\circ} \mathrm{C}$ incubator, $5 \% \mathrm{CO}_{2}$, supernatants were collected, centrifuged at $6000 \mathrm{~g}$ for $30 \mathrm{sec}$ at room temperature, and triplicate conditions combined. Finally, supernatants were filtered by passing them through $0.22 \mu \mathrm{m}$ filter, aliquoted and stored at $-80{ }^{\circ} \mathrm{C}$ until use.

Imagestream Assays: A stock of neutrophils $\left(10 \times 10^{6}\right.$ cells $\left./ \mathrm{mL}\right)$ was cultured at $37^{\circ} \mathrm{C}$, $5 \% \mathrm{CO}_{2}$ in media (RPMI-1640 with L-glutamine), or with opsonized $F$. alocis (MOI 10) for 1,18 , or 24 hours.

For phagocytic experiments, $4 \times 10^{6}$ neutrophils $/ \mathrm{mL}$ from the aged neutrophil stocks were challenged with human serum opsonized Alexa Fluor 488 heat-killed $S$. aureus (Invitrogen; MOI 10) in a shaking water bath at $37{ }^{\circ} \mathrm{C}$ for 30 min. After this timepoint, neutrophils were pelleted at $6000 \mathrm{~g}$ for 30 seconds followed by fixing in the dark with $4 \%$ paraformaldehyde for 10 min. After washing the cells with PBS, the membranes of neutrophils were stained for 10 minutes with Alexa Flour 647-conjugated wheat germ agglutinin ( $2 \mu \mathrm{g} / \mathrm{mL}$, WGA, Molecular probes). Excess WGA was washed off with PBS and the cells were read on the imaging flow cytometer. Phagocytosis was determined using the internalization wizard from the IDEAS software.

For ROS production assays, $4 \times 10^{6}$ neutrophils $/ \mathrm{mL}$ from the aged neutrophil stocks were put into clean microcentrifuge tubes and $2^{\prime}$, 7'-dichlorofluorescein (DCF, $\left.5 \mu \mathrm{M}\right)$ was added to each tube for 10 minutes in a shaking water bath at $37^{\circ} \mathrm{C}$. Half of the volume from each tube was aliquoted into a new microcentrifuge tube: one was used to measure 
basal ROS production and the other one was further challenged with human serum opsonized Alexa Fluor 488 heat-killed S. aureus (Invitrogen; MOI 10) for 30 min. After 30 minutes, the cells were washed with $0.05 \%$ sodium azide and fixed with $1 \%$ paraformaldehyde. Samples were read on the imaging flow cytometer and analysis completed on the IDEAS software. First, the percentage of cells that were positive for $S$. aureus was determined. Next, the mean fluorescence intensity of DCF was quantified for these $S$. aureus positive cells.

Statistical Analysis: Statistical differences among experimental conditions and time points were analyzed using GraphPad Prism Software (Graphpad San Diego, CA, USA). Differences were considered significant at the level $\mathrm{P}<0.05$. The specific statistical test for each experiment is listed in their respective figure legend. If results represent data from multiple experiments, mean values \pm standard error of mean (SEM) are shown.

\section{$\underline{\text { Results: }}$}

F. alocis extends neutrophil lifespan

One of the distinguishing features of neutrophils is their unique nuclear morphology. In viable neutrophils, the nucleus is a multi-lobular structure that resembles flower petals, but as the cells age and undergo constitutive apoptosis, their nucleus will condense into a dense, round shape; therefore, I tested if opsonized $F$. alocis affects nuclear morphology as a way to asses viability (Figure 4.1A). Cytospins of freshly isolated cells showed the distinctive multi-lobular nuclear structure of neutrophils, and culture of neutrophils in media with no serum for 24 hours resulted in the condensation of nuclei in 
the majority of cells. Similarly, addition of the pro-apoptotic translation inhibitor, cycloheximide $(\mathrm{CHX})$, resulted in the majority of neutrophils undergoing changes in nuclear morphology. However, neutrophils co-cultured with F. alocis at a multiplicity of infection (MOI) of 10 bacteria per neutrophil resulted in the retention of nuclear shape after 24 hours.

This finding was confirmed by measuring via flow cytometry the cell surface expression of phosphotidyl serine (PS) and nuclear membrane permeabilization using Annexin V and 7-AAD, respectively (Figure 4.1B). Flow cytometry dot plots show that freshly isolated cells are negative for both 7-AAD and Annexin V (Q4: Annexin V, 7AAD). However, as cells enter early apoptosis, they will become positive for Annexin V (Q3: Annexin $\left.\mathrm{V}^{+}, 7 \mathrm{AAD}^{-}\right)$followed by becoming double positive for both stains (Q2: Annexin $\mathrm{V}^{+}, 7 \mathrm{AAD}^{+}$) during the late stages of apoptosis. Necrotic cells would only be positive for 7-AAD staining (Q1: Annexin $\left.\mathrm{V}^{-}, 7 \mathrm{AAD}^{+}\right)$. When neutrophils are cultured in media for 24 hours, few cells are in the viable double negative quadrant, the majority of neutrophils became Annexin $\mathrm{V}$ positive, and a few cells advanced further into the double positive quadrant, indicating that a large proportion of neutrophils became apoptotic. The CHXtreated cells almost entirely advanced into the apoptotic quadrants, but in $F$. alocis-treated cells, a large fraction of cells remained viable (Q4: Annexin $\left.\mathrm{V}^{-}, 7 \mathrm{AAD}^{-}\right)$. Moreover, almost no cells were in the late apoptosis quadrant $\left(\mathrm{Q} 2\right.$ : Annexin $\left.\mathrm{V}^{+}, 7 \mathrm{AAD}^{+}\right)$or the necrotic cell quadrant (Q1: Annexin $\mathrm{V}^{-}, 7 \mathrm{AAD}^{+}$; Fig. 4.1.B). When the percentages of cells in the viable (Q4: Annexin $\left.\mathrm{V}^{-}, 7 \mathrm{AAD}^{-}\right)$quadrant were combined from 4-6 independent experiments, there was a significantly larger percentage of $F$. alocis- stimulated cells that remained viable as compared to their media cultured-counterparts (Figure 4.1C). 
Furthermore, by cytospin (Figure 4.1D) and flow cytometry (Figure 4.1E), the $F$. alocis-dependent prolonged viability of neutrophils appears to be dose dependent, since using a larger MOI of 50 increased the percentage of cells that retained their nuclear morphology and stained negative for Annexin V and 7-AAD. At a MOI of 100, there was still a significant percentage of viable cells compared to media-treated cells, but not larger than a MOI of 50 (Figure 4.1 D-E). To continue the characterization of this response, I sought to determine if extending neutrophil lifespan was an active process by $F$. alocis. Thus, neutrophils were cultured in media alone or media with CHX, F. alocis, or heatkilled $F$. alocis for 24 hours followed by assessment of apoptosis by cytospin (Figure 4.1F) and flow cytometry (Figure 4.1G). Based on nuclear morphology, heat-killed F. alocis can delay apoptosis as well as the viable bacterium (Figure 4.1F). By flow cytometry, culture of neutrophils with heat-killed $F$. alocis elevated the percentage of cells that were viable, but this number was slightly less than viable $F$. alocis (Figure 4.1G). Together, this data indicates that whether its viable or not, F. alocis can induce a dose-dependent delay in the constitutive apoptosis of neutrophils.

\section{F. alocis induces changes in the transcriptome to extend neutrophil lifespan}

Based on the analysis of the RNAseq data, $11 \%$ of differentially expressed genes are functionally categorized under the cellular process of apoptosis (Figure 2.2). For this chapter, these genes were pooled for a heatmap to assess global changes in their expression (Figure 4.2). The expression of several members of the inhibitor of apoptosis (IAP) family like BIRC8, XIAP, cIAP1/2, Livin, NAIP was differentially regulated. Moreover, members of the extrinsic pathway of apoptosis were downregulated, including caspase 8 and FADD. 
Notably, caspase 9 of the intrinsic pathway was also downregulated. Together, this data indicates that $F$. alocis may be acting on multiple branches of apoptosis.

From the pathogens that can extend neutrophil lifespan, only Anaplasma phagocytophilum can exert anti-apoptotic effects when it is viable and heat-killed [294]. Borjesson et. al published a microarray study of human neutrophils that were challenged with A. phagocytophilum for 1, 3, 6, 9, 12, and 24 hours [294]. Based on the microarray data, they compiled a list of genes upregulated and dowregulated by A. phagocytophilum that relate to apoptosis and cell fate. Using that list, I built a heatmap to assess whether these genes were also differentially expressed in our dataset (Figure 4.3). From our dataset, the genes upregulated by A. phagocytophilum were also upregulated by $F$. alocis and the genes downregulated by A. phagocytophilum were also downregulated by $F$. alocis, suggesting $F$. alocis may be inducing a similar mechanism to extend neutrophil lifespan.

\section{F. alocis induces changes in the expression of pro-survival proteins}

Neutrophil survival relies heavily on the expression of the MCL-1, an antiapoptotic member of the Bcl-2 family [423]. MCL-1 has a short half-life and requires continuous synthesis to carry out its pro-survival effects; however, based on the RNAseq data, the gene expression of MCL1 is significantly decreased in F. alocis stimulated cells (Figure 4.4A). Next, whole lysates from neutrophils cultured in media, with the extrinsic apoptosis inducer Fas ligand (FasL) or with $F$. alocis for $0,3,6,12$, or 24 hours were run on western blots, probed for MCL-1, and the intensity of the band quantified by densitometric analysis (Figure 4.4B-C). Media-cultured cells had a steady, time-dependent decrease in MCL-1protein levels, while treating the cells with FasL accelerated the 
degradation of MCL-1. However, despite not showing statistical significance, cells treated with $F$. alocis had a milder decrease in the expression of MCL-1. These results suggest that while $F$. alocis may not be increasing the expression of MCL-1 at the level of transcription, it protects the anti-apoptotic protein from degradation to retain a pro-survival phenotype.

Another important pro-survival protein in neutrophils is XIAP, which binds to caspase 3 to inhibit its activation. Gene expression data from the RNAseq dataset showed that after 3 and 6 hours of challenge, F. alocis induces the upregulation of XIAP in human neutrophils (Figure 4.5A). The gene expression was matched at the protein level where XIAP was more abundant in cells that were challenged with $F$. alocis that those cultured in media alone (Figure 4.5B-C). The increased XIAP protein expression was detectable as early as 3 hours and remained significantly elevated until 12 hours. Due to the augmented expression of XIAP, I hypothesized that the activation of the executioner protease, caspase 3 may be impaired in F. alocis-treated cells.

\section{F. alocis dampens Caspase 3 activation}

To determine activation of the executioner caspase, western blotting was used to measure the amount of inactive zymogen pro-caspase 3 and active cleaved caspase 3 forms in neutrophils cultured in media or stimulated with FasL or F. alocis during a time course of 3, 6, 12, 24 hours (Figure 4.6A-B). Media treated cells showed a time-dependent decrease in pro-caspase 3 and an increase in the expression of the cleaved caspase 3 fragment, which was faintly detectable at 6 hours. FasL treatment accelerated the accumulation of cleaved caspase 3, which was detectable as early as 3 hours of stimulation. 
On the other hand, F. alocis-challenged neutrophils had dimly detectable cleaved caspase 3 only until 12 hours, which suggests that $F$. alocis delays caspase 3 cleavage.

While processing of procaspases into their mature forms is essential for their activation, it not sufficient to induce maximal activity because their enzymatic function is further modulated through association of cytoplasmic regulatory factors [424]. Thus, the direct activity of caspase 3 was tested in the same experimental conditions by two different assays: one with a chemiluminescent output and the other with a fluorescence output. The theory behind both is similar in that at each timepoint desired, neutrophils will be incubated with a probe that contains a specific cleavage site for active caspase 3 . When caspase 3 cleaves the probe, luminescence or fluorescence will be emitted, and thus the intensity of the signal can be correlated to enzyme activity. Like all previous experiments in this chapter, the stimulation of neutrophils with $F$. alocis was done in suspension for the chemiluminescence-based assay (Figure 4.6C). The kinetics of cleavage of caspase 3 correlated closely with enzyme activity, since FasL-induced activation of caspase 3 had measurable enzyme activity between 3 and 6 hours, with a steep incline in activity after that. Media-cultured cells had caspase 3 cleavage as early as 6 hours and in the activity assay, caspase 3 produced the first increase in light production between 6 and 12 hours. On the other hand, $F$. alocis induced mild caspase 3 cleavage that resulted in significantly diminished protease activity as compared to media or FasL stimulated cells at 12 and 24 hours (Fig 4.6 C).

When infections are carried out in suspension, the output is an average of the responses of neutrophils at different stages in their bacterial interaction kinetics: some may have not interacted with bacteria yet, others may be in the process of phagocytosis, and 
other may have already engulfed and degraded their cargo. Therefore, for the fluorescencebased assay, the neutrophils were attached on glass coverslips and the infection with $F$. alocis was synchronized through a centrifugation step (Figure 4. 6D-E). Overall, the trend of caspase activity was similar to the chemiluminescence assay, where $F$. alocis induced decreased caspase 3 activity. However, it is important to note that adhesion in itself prolongs the lifespan of neutrophils [425], so the timing of caspase 3 activation is delayed in all conditions, and why I assessed caspase 3 activity as late as 48 hours. In conclusion, F. alocis delays the cleavage and activation of procaspase 3 , followed by a decrease in caspase 3 activity that ultimately results in prolonged neutrophil survival.

\section{F. alocis delays DNA fragmentation}

The final outcome of caspase 3 activation is the destruction of cellular structures like cytoskeletal protein degradation or DNA fragmentation [426]. Specifically, during the final stages of apoptosis, caspase3-activated DNAses cleave nuclear DNA, which can be detected experimentally through terminal deoxynucleotidyl transferase dUTP nick end labeling (TUNEL) staining. TUNEL staining will fluorescently label exposed 3'hydroxyl ends that occur as a result of DNA strand cleavage, and the increase in fluorescence can be detected via flow cytometry. Using this technique, I tested DNA fragmentation in our experimental conditions (Figure 4.7A-B). Representative flow cytometry histograms from each condition were superimposed to show differences in DNA fragmentation at 6-24 hours of culture (Figure 4.7A). At 6 hours, the majority of untreated and F. alocis stimulated neutrophils were negative for DNA fragmentation like freshly isolated cells. However, as the timecourse progressed, a larger population ( 40\%) of media-cultured 
neutrophils became positive for the TUNEL probe at 12 hours, whereas only $20 \%$ of $F$. alocis treated neutrophils had detectable DNA fragmentation (Figure 4.7B). The difference in these values increased to statistical significance at 24 hours, where $\sim 60 \%$ of media neutrophils became positive for TUNEL while only $\sim 40 \%$ F. alocis stimulated cells were TUNEL positive. By 48 hours of ex-vivo culture, all cells became TUNEL +. Collectively, this data supports the idea that relative to untreated cells, F. alocis markedly delayed DNA fragmentation to retain neutrophil viability.

\section{F. alocis meekly activates initiator caspases}

Caspase 8 is the initiator caspase of the extrinsic apoptosis pathway that is initiated upon ligation of surface death receptors and caspase 9 is the driver of the intrinsic pathway that is activated through the release of mitochondrial factors into the cytoplasm [392]. Having established that caspase 3 activation is delayed in neutrophils stimulated with $F$. alocis, I sought to determine which pathway is triggered upstream of caspase 3 activation through the same chemiluminescence-based activity assays employed to detect caspase 3 activation. In untreated cells, caspase 8 (Figure 4.8A) and caspase 9 (Figure 4.8B) activity peaked between 12 and 24 hours. Fas L was used as an extrinsic apoptosis pathway activator and staurosporine was used as an inducer of the intrinsic apoptotic pathway. Both of these positive controls induced activity of caspase 8 and -9 , respectively, at earlier timepoints than untreated cells. Contrastingly, neutrophils treated with $F$. alocis had a gradual minimal increase in the activity of both caspases. Overall, the activity of caspases 8 and 9 was markedly lower in F. alocis treated cells as compared to untreated cells, which suggests that the oral pathogen may be influencing the apoptotic cascade at multiple points. 
Furthermore, since caspase 3 activation and DNA fragmentation is detected at 24 hours of culture with $F$. alocis, it is possible that caspase 3 is being activated through an alternative, initiator caspase independent mechanism.

F. alocis pro-survival effect depends on contact with neutrophils through TLR2/6

Since neutrophil programmed cell death can be delayed by host derived factors like DAMPS and pro-inflammatory cytokines or bacteria-derived components, I tested whether contact with the bacterium is necessary to induce the prolonged lifespan of neutrophils. First, I conducted a crude flow cytometry Annexin V/7-AAD assay where neutrophils were stimulated with CFSE-labeled $F$. alocis. This allowed for an initial gate where neutrophils were separated by their CFSE intensity as a measure of association with $F$. alocis (Figure 4.9A). Here, the neutrophils were divided into two populations: F. alocis negative (low CFSE) or F. alocis positive (high CFSE), which were roughly the same size. Then, these two populations were plotted in a traditional Annexin V/7-AAD quadrant to assess their apoptotic state. It is possible to see from the flow cytometry dot plots that F. alocis (-) neutrophils progressed into early apoptosis $\left(\mathrm{Q} 3\right.$ : Annexin $\left.\mathrm{V}^{+}, 7-\mathrm{AAD}^{-}\right)$at a higher percentage than $F$. alocis $(+)$ cells, and that a larger portion of $F$. alocis $(+)$ cells remained in the viable quadrant $\left(\mathrm{Q} 4\right.$ : Annexin $\left.\mathrm{V}^{-}, 7-\mathrm{AAD}^{-}\right)$. This viability trend reached statistical significance when two independent experiments were combined (Figure 4. 9B), suggesting that association with $F$. alocis directly enhances neutrophil viability.

To determine if manipulation of the neutrophil phenotype required the binding or phagocytosis of $F$. alocis, I used transwells with a pore size of $0.4 \mu \mathrm{m}$ to quarantine the neutrophils (PMN) from the bacteria (Figure 4. 9C). When neutrophils and F. alocis were 
in the same compartment, the viability of neutrophils increased as previously shown. However, this effect was reversed when the oral pathogen and neutrophils were separated by the transwell's filter, confirming that contact between both parties is essential to induce the pro-survival phenotype.

Since $F$. alocis is recognized by TLR2/6 receptors to initiate numerous neutrophil effector functions, I assessed if the apoptosis delay was also mediated through TLR2/6 (Figure 4.9D). For these experiments, neutrophils were pre-treated with neutralizing antibodies against TLR2 and TLR6 or corresponding isotype controls prior to stimulation with $F$. alocis for 24 hours. As expected, CHX treatment decreased neutrophil viability and F. alocis increased it. Blocking access to TLR $2 / 6$ decreased viability comparable to basal levels, while the isotype control did not affect the $F$. alocis driven survival effect. Combined, this data demonstrated that $F$. alocis contact with neutrophils through TLR2/6 ligation is responsible for the prolonged neutrophil lifespan.

\section{Factors released during $\mathrm{F}$. alocis \& neutrophil interaction are pro-survival}

Although contact of neutrophils with $F$. alocis through TLR2/6 is essential to initiate neutrophil survival, the pro-survival response could be due to a paracrine effect from cytokines or other pro-survival factors released by neutrophils downstream of TLR2/6 ligation. Therefore, to test this possibility, the supernatants of neutrophils cultured in media or stimulated with F. alocis for 24 hours were collected and sterile filtered to generate conditioned media (Figure 4.10A). Then, naïve neutrophils were cultured in fresh or conditioned media for 24 hours and their apoptosis status assessed by cytospin (Figure 4.10B). Neutrophils cultured in conditioned media from untreated cells developed nuclear 
condensation whereas the multi-lobulated nuclear morphology was retained in neutrophils cultured in the conditioned media from $F$. alocis-stimulated cells. This was confirmed by flow cytometry where the progression into apoptosis of cells in the conditioned media versus direct stimulation in fresh media was compared (Figure 4.10C). Both sets on untreated cells, cultured in fresh or conditioned media, had the same percentage of viable cells. When neutrophils were cultured with $F$. alocis-conditioned media, there was an increase in the number of viable cells as compared to untreated cells, which indicates that the interaction between $F$. alocis and neutrophils generates pro-survival factors. However, this percentage of viable cells was not as robust as when neutrophils are stimulated with $F$. alocis in fresh media, suggesting that both the bacteria-neutrophil interaction and the secreted pro-survival factors are necessary to induce maximal neutrophil survival.

To determine the contribution of TLR2/6 signaling to the generation of these prosurvival factors, a new set of conditioned media was collected where neutrophils were pretreated with neutralizing antibodies against TLR2/6 or isotype controls prior to stimulation with $F$. alocis for 24 hours. The supernatants were collected, sterile filtered, and used to culture naïve neutrophils for 24 hours (Figure 4.10D). Assessment of apoptosis via flow cytometry determined that while culturing cells in the $F$. alocis-conditioned media is not as potent as stimulation with $F$. alocis, when neutrophils are pre-treated with inhibitors for TLR2/6, the pro-survival effect is lost completely. Therefore, I conclude that the contact between neutrophils and $F$. alocis through TLR2/6 initiates a signaling cascade that produces pro-survival factors with paracrine effects on non-infected cells. However, this pro-survival soup is not sufficient to elicit the maximal neutrophil survival possible in this context and requires the presence of the bacterium. 
Neutrophils with F. alocis-mediated survival retain their functional capacity

As a non-inflammatory type of cell death, cells undergoing apoptosis have restricted proinflammatory capacities like an impairment in chemotaxis, phagocytosis and degranulation [392]. Since neutrophils challenged with $F$. alocis remain viable for longer periods of time, I sought to determine whether they were also still capable of performing their effector functions. Neutrophils were aged in media or media with opsonized $F$. alocis for 18 or 24 hours, followed by the addition of non-viable, fluorescent $S$. aureus at a MOI of 10 bacteria per neutrophil. These $S$. aureus particles are known to be ingested easily and induce a robust ROS generation. The phagocytic capacity of these aged cells was tested via imaging flow cytometry after fluorescently labeling the neutrophil membrane. Fig. 4.11A shows representative neutrophils that were classified as $S$. aureus negative and $S$. aureus positive. At 18 hours of culture, F. alocis-challenged neutrophils were significantly more efficient at phagocytizing the opsonized S. aureus (Figure 4.11B). The neutrophils from the same donors were also tested after 24 hours, and while the trend was the same, there was no statistical significance between media-aged neutrophils and their F. alocischallenged counterparts.

Using imaging flow cytometry, the ability of aged neutrophils to produce ROS was also assessed. The culture protocol was the same as above, but this time, aged neutrophils were incubated with 2',7'-dichlorodihydrofluorescein diacetate (DCF) prior to S. aureus challenge. The ROS-reduced form of DCF will emit fluorescence, so increases in the intensity of fluorescence can be attributed to ROS production, which is demonstrated in the representative images (Figure 4.11C). By comparing the mean fluorescence intensity 
(MFI) of DCF at 1 hour, there is no significant difference between media or F. alocis cultured neutrophils. However, as the cells age for 18 hours, the untreated cells lose some of their capacity to produce ROS against $S$. aureus whereas $F$. alocis stimulated cells produced a significantly higher amount of ROS (Fig 4.11 C-D). This difference was lost at 24 hours, where $F$. alocis cultured cells also begin to lose their functional capacity. Thus, it becomes clear that $F$. alocis-challenged neutrophils were able to also retain their ability to produce ROS for longer timepoints than media treated cells. Ultimately, this prolonged lifespan and functional capacity could result in increased neutrophil-driven inflammation, which is a hallmark of periodontal disease.

\section{Discussion:}

Despite their reputation as "pathogen busters," neutrophil functions extend beyond eliminating invading bacteria. Neutrophils will undergo controlled cell death through apoptosis to diminish their pro-inflammatory potential and become targets for efferocytic clearance by macrophages. The clearing of spent neutrophils initiates inflammation resolution protocols and tissue repair; in fact, neutrophil apoptosis at sites of infection is a characteristic of effective immune response [427]. In periodontitis, there is an extensive accumulation of neutrophils, which are found at different stages of cell death [210, 264, 428]. This suggests that there is modulation of neutrophil lifespan and/or a defect in the efficient clearing of dying neutrophils. However, there are only a few publications that have addressed neutrophil viability after encounters with oral bacteria, and they have focused on extremely short timepoints (three hours or less). Since the majority of studies on cell death after interaction with periodontal pathogens have been done on epithelial cells 
and macrophages, this work fills an important gap in the knowledge of the scientific community. To this point, $F$. alocis challenge induces apoptosis in normal oral keratinocytes and epithelial cells $[67,73]$.

To assess apoptosis in neutrophils, I chose to use serum-free media in our experimental design to eliminate the confounding effects of growth factors and serum components as previously described [407, 429]. Even in these limiting conditions, F. alocis was able to delay neutrophil constitutive apoptosis pathways. Specifically, our results show that relative to control cells, neutrophils challenged with $F$. alocis retained their nuclear morphology, showed decreased phosphatidylserine externalization by Annexin V staining, and had diminished DNA fragmentation as tested by TUNEL. This phenotype could be explained by a marked delay in the processing and subsequent activity of the executioner caspase, caspase 3; an effect that has been previously observed for other intracellular pathogenic bacteria like Francisella tularensis [407]. It is likely that in the case of F. alocis challenged neutrophils, the delayed processing of caspase 3 is partially linked to the increased gene and protein expression of XIAP, a protein that physically binds to Caspase 3 and prevents its activation. This is similar to the mechanism deployed by Neisseria gonorrhoeae, which transiently delays the onset of apoptosis in parallel to the upregulation of cIAP2 and XIAP [430]. Compounding on this inhibitory effect was a defect in the activity of initiator caspases 8 and 9 , suggesting that $F$. alocis is able to modulate the activation of the intrinsic and extrinsic pathways. It would be important to also test the processing of both these caspases via western blotting, as was done with Caspase 3 to determine where $F$. alocis is impacting the activity of these caspases. 
Of note, all of these apoptosis assays were conducted under aerobic conditions, where the presence of oxygen inactivates the majority of $F$. alocis in the culture between 4 and 10 hours (Shown in Chapter 6). Incidentally, after 6 hours of culture, the levels of XIAP begin to decrease, procaspase 3 is cleaved, and DNA fragmentation becomes detectable. While this suggests that viable $F$. alocis may be actively inducing XIAP expression, heat-killed $F$. alocis also had a net pro-survival phenotype. This was surprising given the fact that heat-killed $F$. alocis results in efficient phagosome maturation and induces robust ROS production, which is an initiating factor in the phagocytosis-induced cell death program. Our results also demonstrated that contact with $F$. alocis is required for the pro-survival effect to take place. Both viable and heat-killed $F$. alocis activate TLR2/6, which was necessary to induce neutrophil survival. Furthermore, the conditioned media from neutrophils stimulated with $F$. alocis had pro-survival effects on naïve neutrophils, likely through the release of pro-inflammatory cytokines that extend paracrine neutrophil survival effects. Other than IL-8 production, the differences in cytokine output after challenge with viable or heat-killed $F$. alocis has not been assessed. Changes in neutrophil signaling between viable and heat-killed $F$. alocis may be responsible for these differences and is an ongoing area of research in our laboratory.

Interaction with intracellular microbes can extend neutrophil lifespan, sometimes to the advantage of the microbial agenda. For example, Chlamydia pneumoniae and the fungal pathogen, Paracocciodes brasiliensis, that delay apoptosis by stimulating neutrophil secretion of IL-8 [431, 432]. The Gram-negative Anaplasma phagocytophilum also delays apoptosis for up to 90 hours [294, 433, 434], and shares similarities with $F$. alocis. Much like F. alocis, A. phagocytophilum reduces proinflammatory cytokine release, 
fails to induce ROS production, and delays apoptosis whether the bacterium is viable or not [435-440]. Additionally, when the differential apoptosis gene expression profile from A. phagocytophilum was assessed in our transcriptome data, the $F$. alocis-induced pattern of gene expression generally mirrored the one from A. phagocytophilum, suggesting the bacteria may have overlapping virulence strategies against neutrophils. It is known that $A$. phagocytophilum inhibits cell apoptosis through the activation of the Janus kinase/signal transducers and activators of transcription (JAK/STAT) pathway and type IV secretion system (T4SS)-secreted proteins [441]; but I have not assessed whether $F$. alocis activates these pathways and it is unknown whether the oral pathogen has a secretion system that is relevant to human infection.

In conclusion, this is the first report to do an in-depth characterization of the apoptotic effect the emerging oral pathogen, F. alocis has on human neutrophils. Although more work will be needed to establish the in vivo relevance of this data, I speculate that the extended neutrophil lifespan will have serious implications in disease progression by extending the window of time where neutrophils can promote dysbiotic inflammation in the oral cavity. 
A.

24 hours
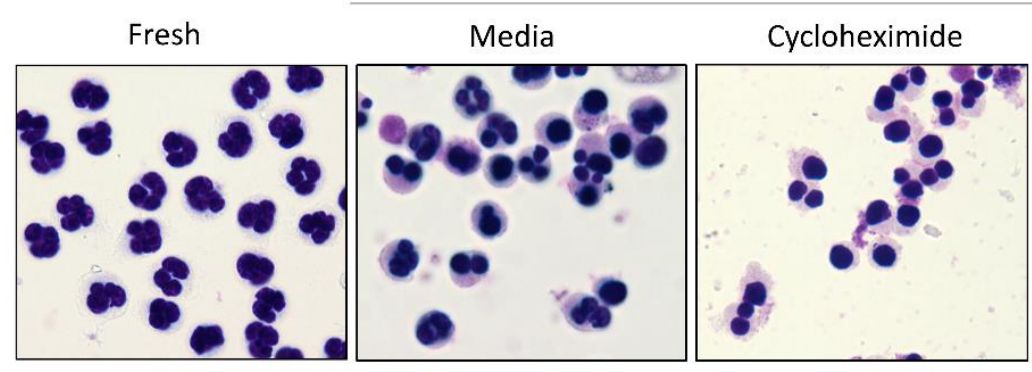

F. alocis

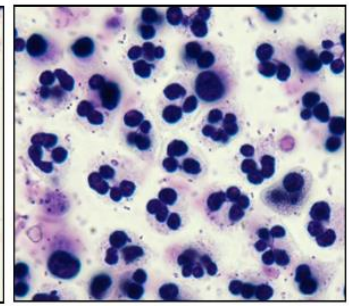

B.

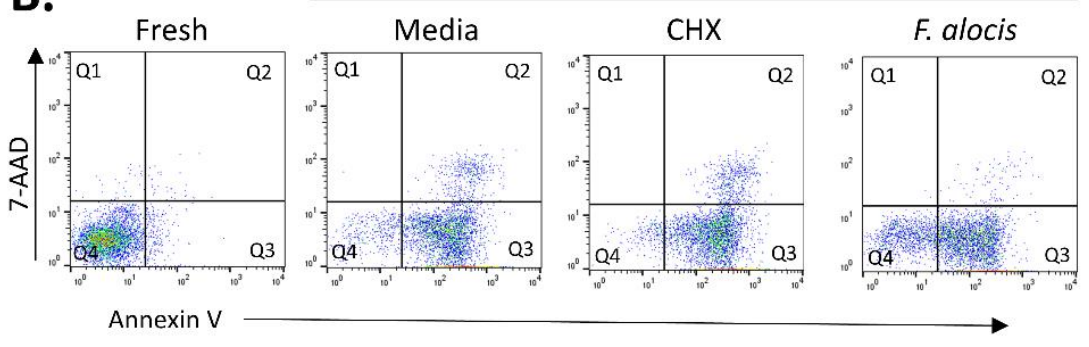

D.

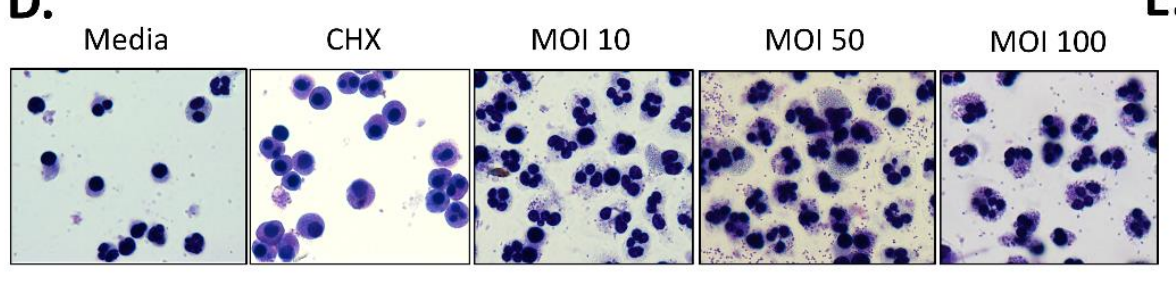

F.

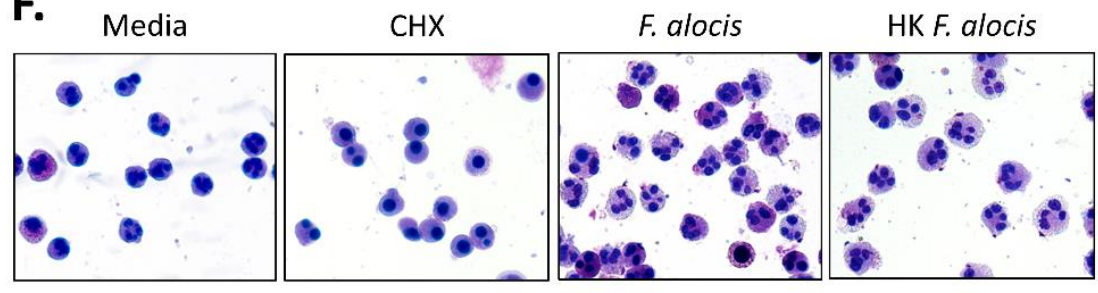

C.

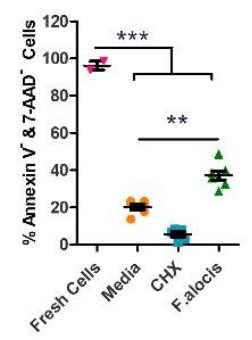

E.

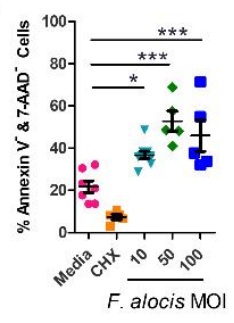

G.

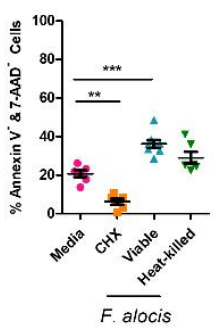

Figure 4.1: F. alocis delays neutrophil apoptosis. 
Figure 4.1: (A, D, F) Nuclear morphology changes were assessed by light microscopy using images of HEMA-stained cytospins from freshly isolated neutrophils, neutrophils cultured in media, or neutrophils stimulated with cycloheximide (CHX), F. alocis (MOI 10, 50 or 100), or heat-killed (HK) F. alocis for 24 hours. (B) Flow cytometry dot plot and gating strategy of AnnexinV/7AAD stained neutrophils treated with the same conditions as A. (C, E, G) Plots shows the \% viable (Q4: Annexin V-, 7AAD-) neutrophils \pm SEM from 2-7 independent experiments. A one-way ANOVA with Tukey post-hoc tests was performed on graphs $\mathrm{C}, \mathrm{E}$, and $\mathrm{G} . * \mathrm{p}>0.05, * * \mathrm{p}>0.01, * * * \mathrm{p}>0.001$. 


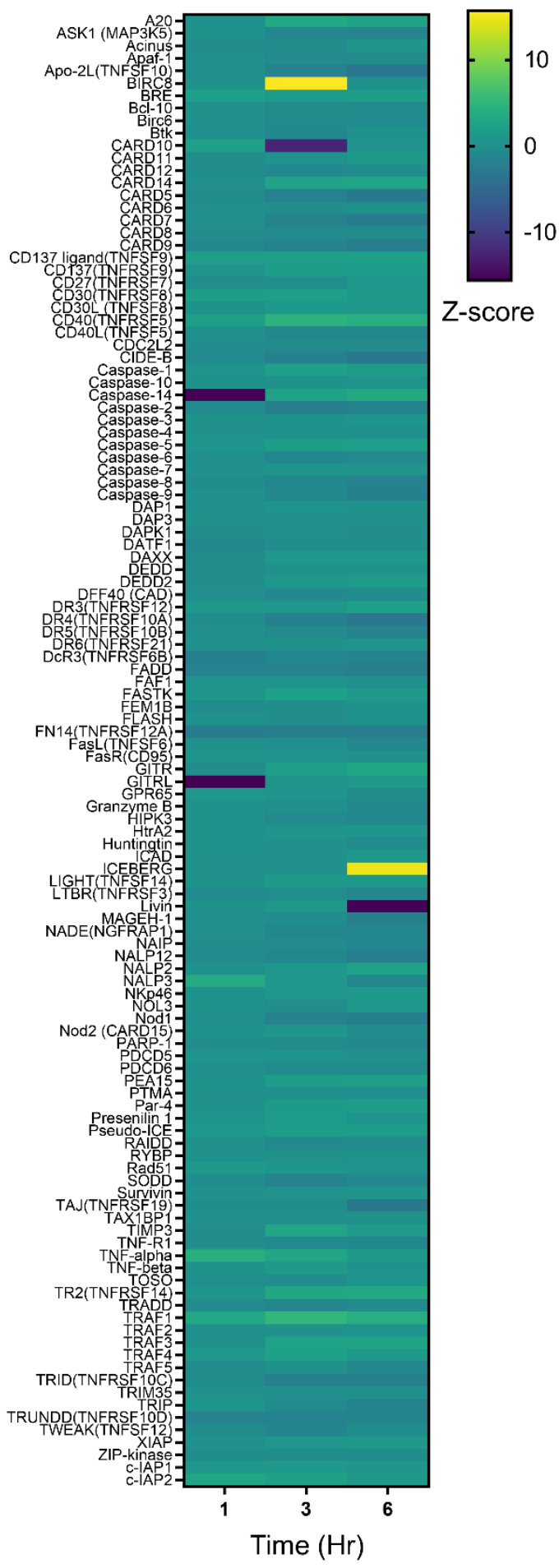

Figure 4.2: $F$. alocis induces changes in gene expression of apoptotic genes. 
Figure 4.2: Heatmap shows the differential expression of genes in the apoptosis process cluster from MetaCore. Gene expression data was generated from Illumina RNA sequencing performed on human neutrophils from 4 individual healthy donors that were either left unstimulated or challenged with $F$. alocis for 1, 3 or 6 hours. 


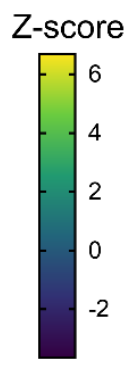

ADORA2A|adenosine A2a recepto
BCL3|B-cell CLL/lymphoma
BCL11A|B-cell CLL/lymphoma 11A (zinc finger protein)
BTG3|BTG family, member 3

CASP2|caspase 2, apoptosis-related cysteine peptidase

GADD45B|growth arrest and DNA-damage-inducible, beta G0S2|G0/G1 switch 2

IER5|immediate early response 5 IER3|immediate early response 3

IER5|immediate early response 5 INHBA|inhibin, beta A JAG1 jagged 1

LIMS1|LIM and senescent cell antigen-like domains 1 SMAD4|SMAD family member 4 NFKBIA|nuclear factor of kappa light polypeptide gene enhancer in B-cells inhibitor, alpha NR4A3|nuclear receptor subfamily 4 , group A, member 3 NSMAF|neutral sphingomyelinase ( $\mathrm{N}$-SMase) activation associated factor PHLDA2|pleckstrin homology-like domain, family A, member 2 PPP1R15A|protein phosphatase 1 , regulatory subunit 15A

TLR2|toll-like receptor 2 TNIP1|TNFAIP3 interacting protein 1 TNFSF14|tumor necrosis factor (ligand) superfamily, member 14 TNFSF15|tumor necrosis factor (ligand) superfamily, member 15 TRAF1|TNF receptor-associated factor 1

ACIN1|apoptotic chromatin condensation inducer 1 FOXN3|forkhead box N3 APAF1|apoptotic peptidase activating factor 1 FOXO3|forkhead box $\mathrm{O3}$

FRAT1|frequently rearranged in advanced T-cell lymphomas 1 MKL1|megakaryoblastic leukemia (translocation) 1

RAD21|RAD21 cohesin complex component SIPA1|signal-induced proliferation-associated 1

STK17A|serine/threonine kinase 17a TACC3|transforming, acidic coiled-coil containing protein 3 TUSC2|tumor suppressor candidate 2
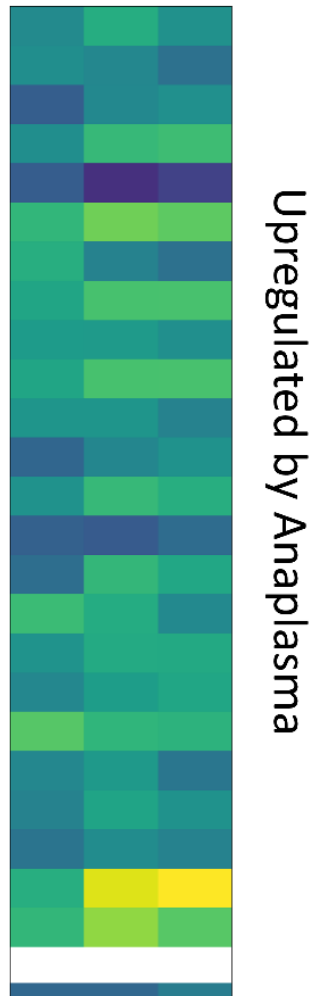

Figure 4.3: $F$. alocis induces a pattern of apoptosis gene expression like Anaplasma

phagocytophilum. A list of upregulated and downregulated genes related to apoptosis was acquired from a microarray study by Borjesson et. al. The expression of these genes was assessed in our data set. The heat map depicts our data from neutrophils challenged with F. alocis for 1,3 or 6 hours, but the genes are clustered based on whether they were upregulated or downregulated by A. phagocytophilum. 

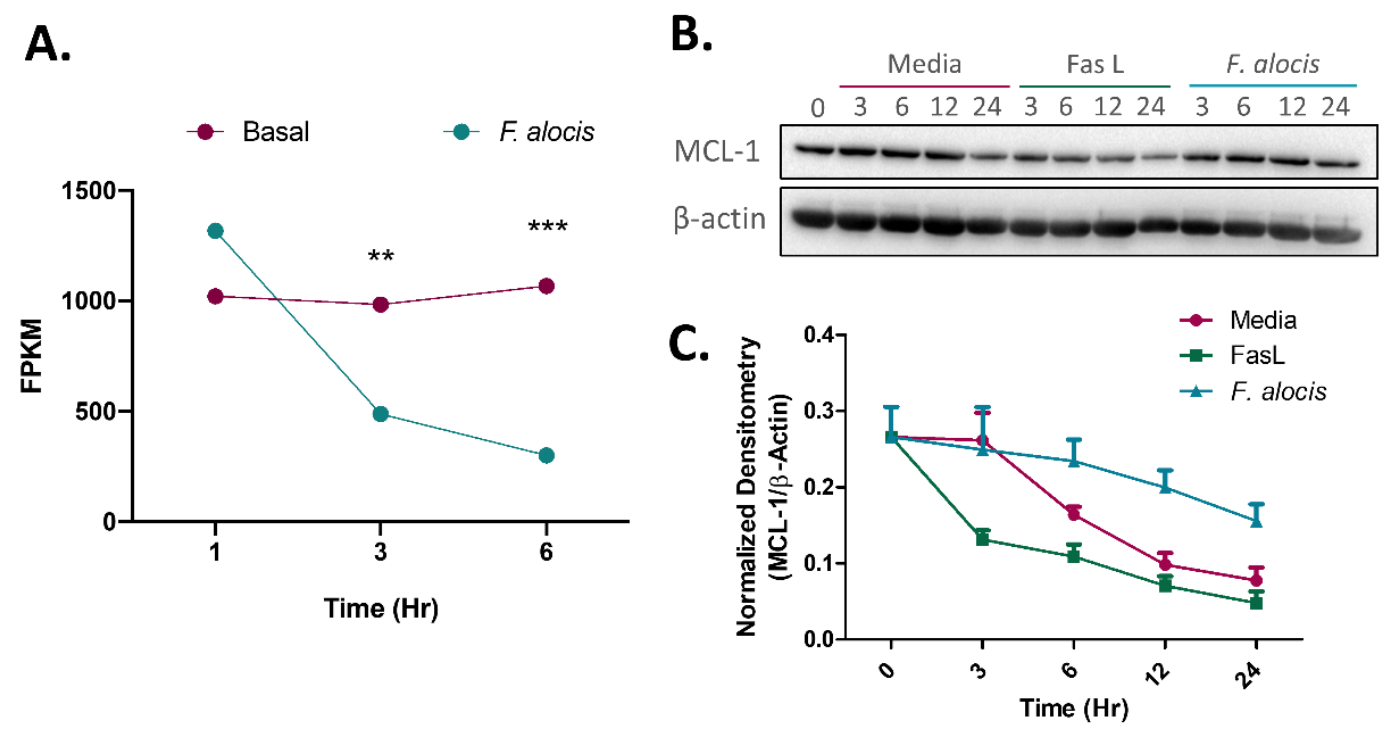

Figure 4.4: F. alocis upregulates gene expression of anti-apoptotic MCL-1 and delays its degradation. Line graphs show the average Fragments Per Kilobase of transcript per Million mapped reads (FPKM) of the gene for MCL-1 (A) in unstimulated (Basal) neutrophils and $F$. alocis challenged neutrophils from 4 healthy donors. Representative images show the western blots of whole cell lysates from media-cultured cells or cells stimulated with Fas L or F. alocis that were probed for MCL-1 (B). Normalized western blot densitometries are summarized from 4 and 7 independent experiments, respectively, and plotted \pm SEM (C). Two-way ANOVAs with Bonferroni post-hoc testing was performed on all graphs. $*=p>0.05, * *=p>0.01, * * *=p>0.001$ 

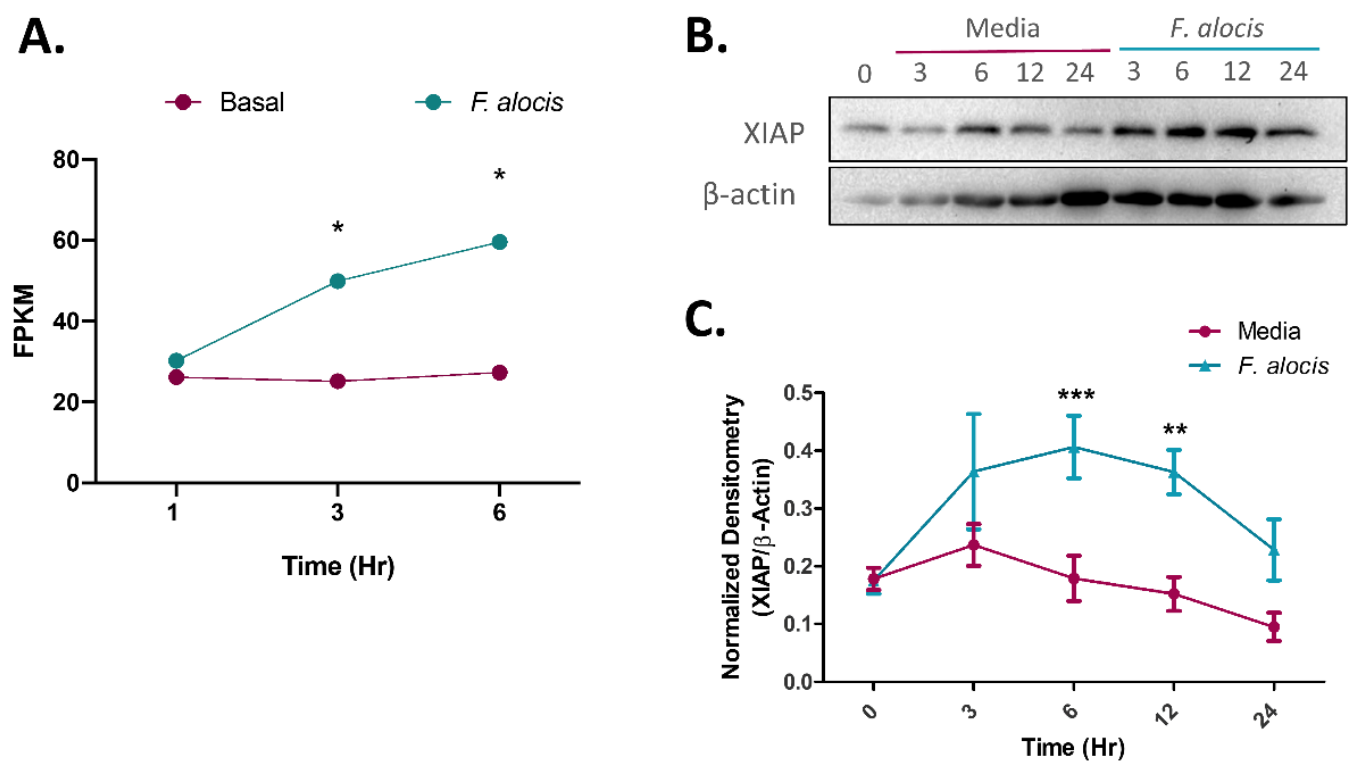

Figure 4.5: $F$. alocis upregulates genes \& protein expression of XIAP. Line graphs show the average Fragments Per Kilobase of transcript per Million mapped reads (FPKM) of the gene for XIAP (A) in unstimulated (Basal) neutrophils and F. alocis challenged neutrophils from 4 healthy donors. Representative images show the western blots of whole cell lysates from media-cultured cells or cells stimulated with Fas L or F. alocis that were probed for XIAP (B). Normalized western blot densitometries are summarized from 4 and 7 independent experiments, respectively, and plotted \pm SEM (C). Two-way ANOVAs with Bonferroni post-hoc testing was performed on all graphs. $*=p>0.05, * *=p>0.01, * * *=$ $\mathrm{p}>0.001$ 
A.
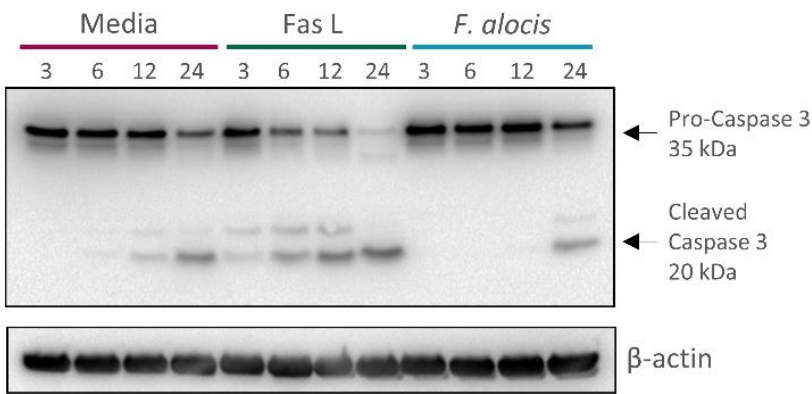

B.

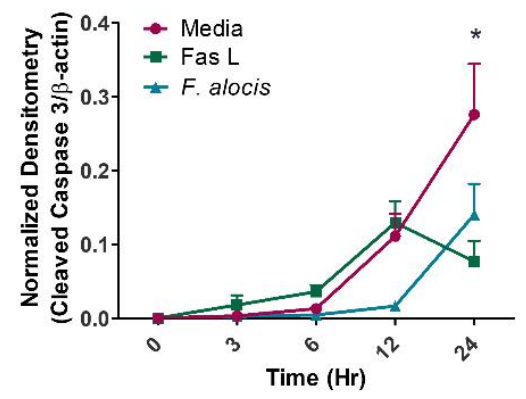

c.

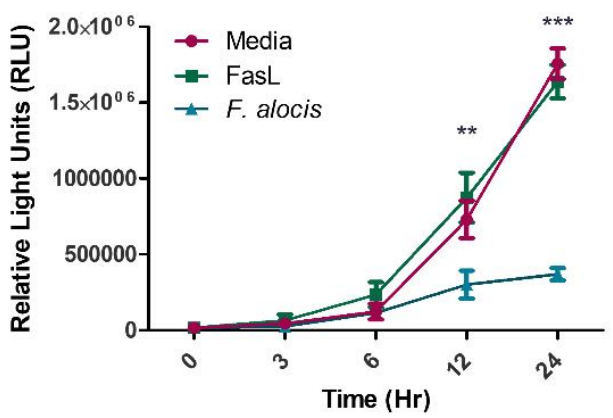

E.

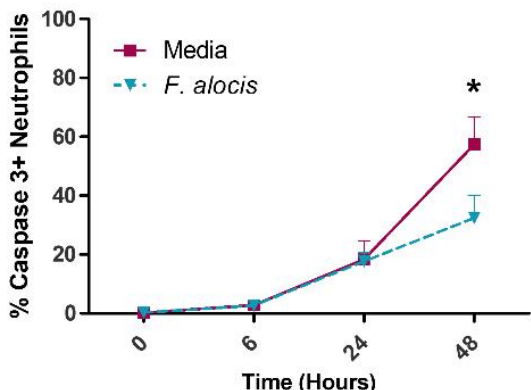

Figure 4.6: $F$. alocis delays Caspase 3 activation. 
Figure 4.6: (A) Representative blot of time-dependent Caspase 3 processing from whole cell lysates of untreated (media), Fas L or F. alocis treated neutrophils. (B) Data are plotted as the mean \pm SEM of the normalized densitometry of cleaved caspase 3 from 8 independent experiments. Asterisk denotes statistical significance in comparison to both $F$. alocis and Fas L. (C) Caspase 3 activity was tested in suspension using a chemiluminescent assay on neutrophils that were untreated (Media) or stimulated with Fas L or F. alocis (MOI 10) for 3, 6, 12 or 24 hours. Data are plotted as the mean \pm SEM of the luminescence produced for each condition from 4 independent experiments. (D) Attached neutrophils in media or challenged with $F$. alocis for $0,6,24$, or 48 hours were examined for caspase activity by a fluorescence-based assay and imaged using confocal microscopy. Cells that express green fluorescence have active caspase 3 activity. (E) 100 cells were counted from each condition and qualified on whether they became fluorescence as a measure of caspase activity. Data are plotted as the percent \pm SEM of neutrophils that became Caspase 3 positive at each timepoint from 5 independent experiments. Asterisk denotes statistical significance between media and F. alocis conditions. A two-way ANOVA with Bonferroni post-hoc testing was performed on all graphs. $*=\mathrm{p}>0.05, * *=\mathrm{p}>0.01, * * *=\mathrm{p}>0.001$ 
A.

Fresh cells Media F. alocis
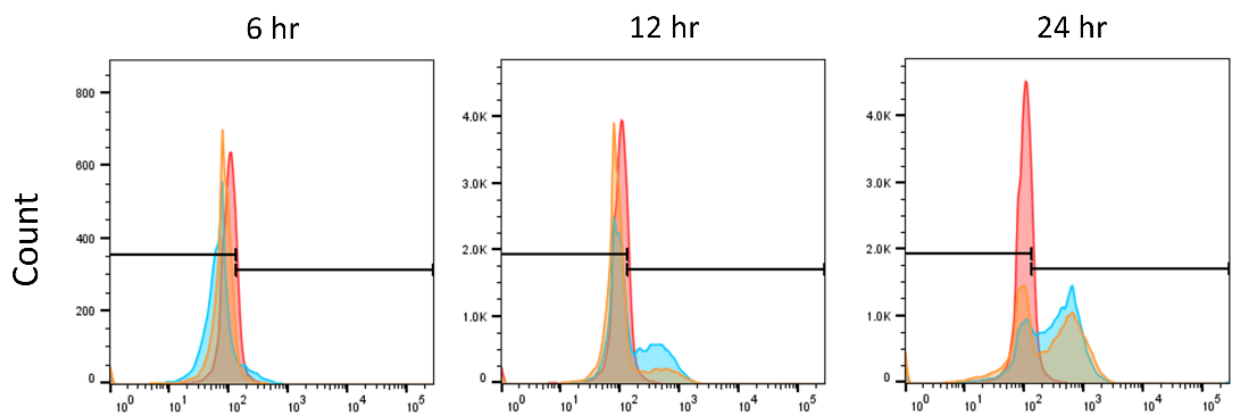

BrdU-FITC

B.

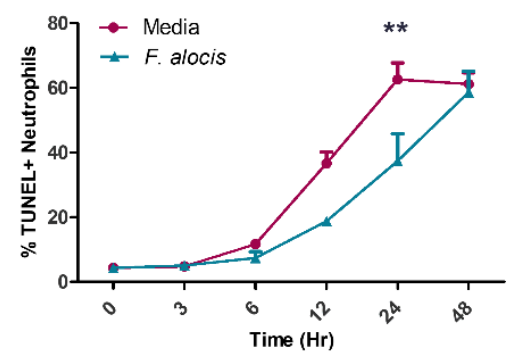

Figure 4.7: $F$. alocis delays DNA fragmentation. A flow cytometry based TUNEL assay was conducted on neutrophils cultured in media or with F. alocis (MOI 10) for 3, 6, 12, 24, and 48 hours. DNA fragmentation was detected as an increase in fluorescence by the BrdU-FITC antibody. (A) Shows flow cytometry histograms of freshly isolated neutrophils, media cultured neutrophils, or neutrophils challenged with $F$. alocis after 6 , 12 , and 24 hours. Gating was made to delineate the rightward shift in the populations that became BrdU positive due to DNA fractionation. (B) The percent of TUNEL+ neutrophils represents the number of cells \pm SEM that migrated into the right gate in 4 independent experiments. A two-way ANOVA with Bonferroni post-hoc testing was performed where $*=\mathrm{p}>0.05, * *=\mathrm{p}>0.01, * * *=\mathrm{p}>0.001$. 
A.

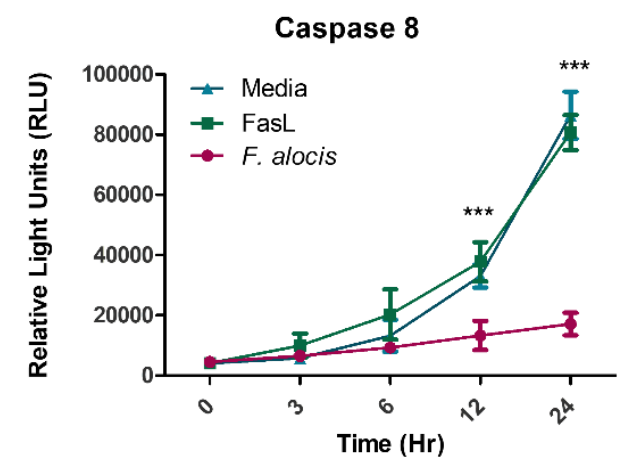

B.

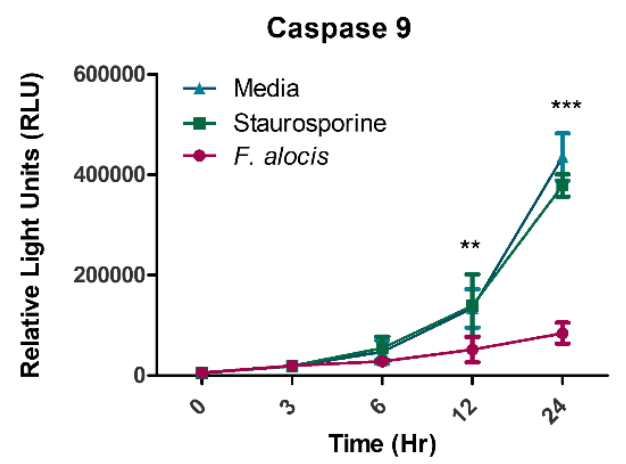

Figure 4.8: $F$. alocis minimally activates the initiator caspases, 8 and 9. Caspase 8 (A) and Caspase 9 (B) activity was tested in suspension using a chemiluminescent assay on neutrophils that were untreated (Media), stimulated with positive controls Fas L or staurosporine, or F. alocis (MOI 10) for 3, 6, 12 or 24 hours. Data are plotted as the mean \pm SEM of the luminescence produced for each condition from 4 independent experiments. A two-way ANOVA with Bonferroni post-hoc testing was performed on A and B where * $=\mathrm{p}>0.05, * *=\mathrm{p}>0.01, * * *=\mathrm{p}>0.001$. 
A.

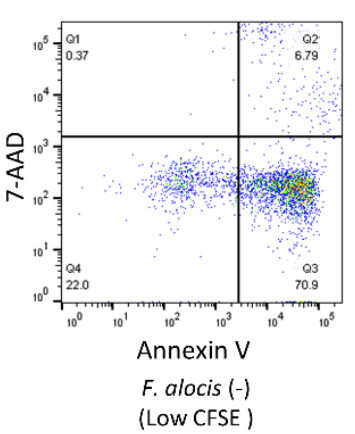

C.
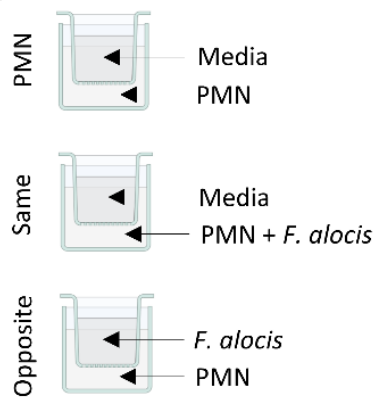

B.

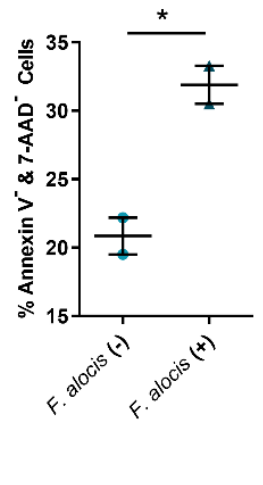

Figure 4.9: Contact with $F$. alocis through TLR2/6 is necessary for prolonged neutrophil lifespan. 
Figure 4.9:Annexin V/7AAD staining was performed on neutrophils that were challenged with CFSE-expressing F. alocis for 24 hours. (A) Shows the flow cytometry gating strategy employed. First the neutrophils were first sorted into $F$. alocis negative or positive based on their CFSE intensity, followed by classical Annexin V/7AAD gating. (B) Shows the mean percent viable (Q4: Annexin $\mathrm{V}-$, 7AAD-) neutrophils \pm SEM from the F. alocis negative or positive cells from two independent experiments. A two-tailed student T-test was performed to determine statistical significance. (C) Neutrophils (PMN) were cultured in transwells as depicted with or without $F$. alocis for 24 hours followed by Annexin V/7AAD staining. Data are plotted as the mean percent viable (Q4: Annexin V-, 7AAD-) neutrophils \pm SEM from two independent experiments. (D) Neutrophils were untreated (Basal) or stimulated with $F$. alocis after pre-treatment with media, neutralizing TLR2/6 antibodies or isotype controls. Data are plotted as the mean percent viable (Q4: Annexin V-, 7AAD-) neutrophils \pm SEM from 6 independent experiments. A one-way ANOVA with Tukey post-hoc testing was performed on C \& D to determine statistical significance. $*=\mathrm{p}>0.05, * *=\mathrm{p}>0.01, * * *=\mathrm{p}>0.001$. 

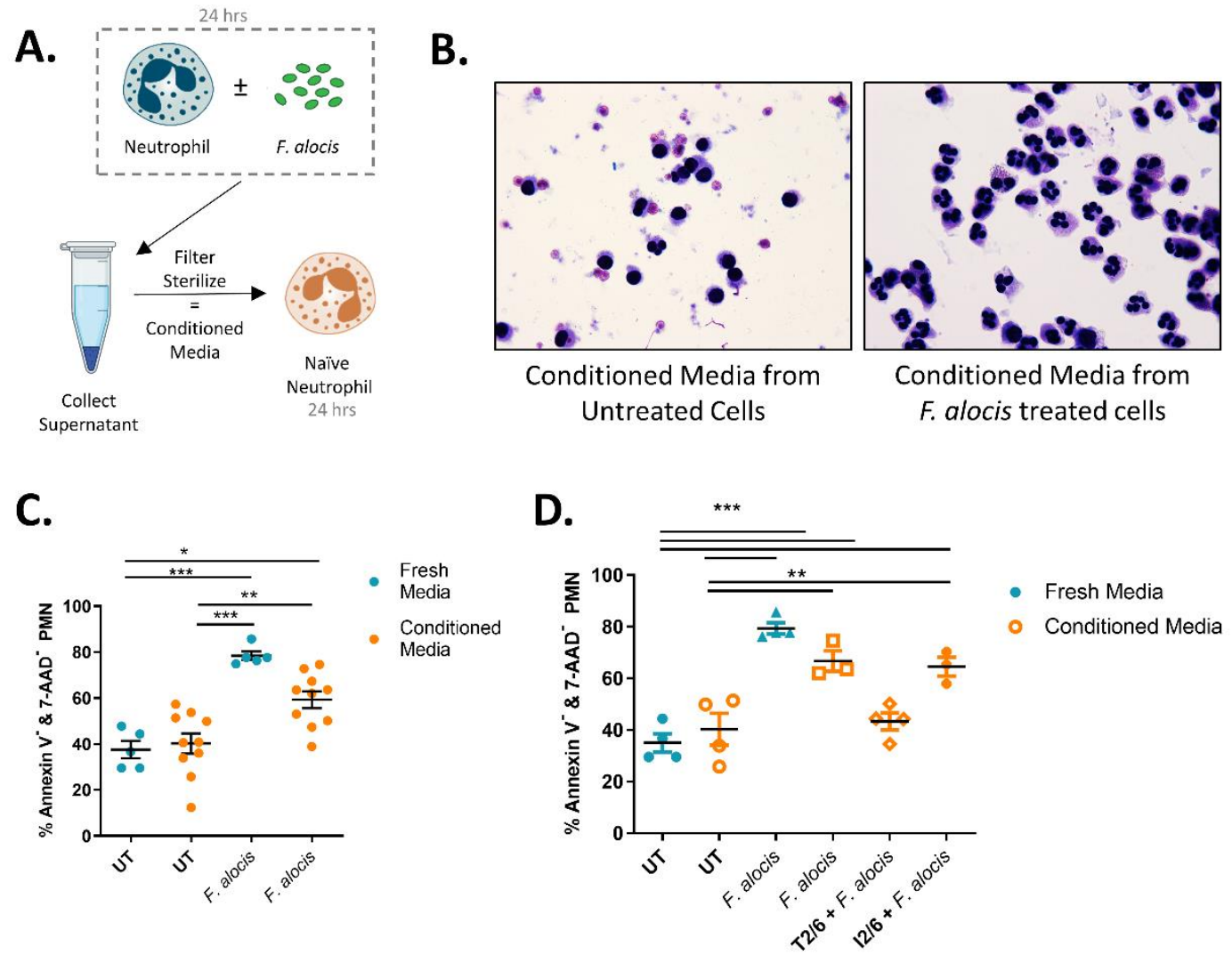

Figure 4.10: Factors released during $F$. alocis \& neutrophil interaction are prosurvival. 
Figure 4.10: (A) Graphic depicting how conditioned media was generated. (B) HEMAstained cytospins of naïve neutrophils cultured in the conditioned media from untreated cells or F. alocis treated cells. (C) Neutrophils were cultured in fresh media (blue dots) and left untreated (UT) or stimulated with $F$. alocis, or cultured in the conditioned media (orange dots) of untreated (UT) of F. alocis-stimulated cells for 24 hours followed by staining for Annexin V and 7AAD. Data are plotted as the mean percent viable (Q4: Annexin V-, 7AAD-) neutrophils \pm SEM from 5-10 independent experiments. (D) Naïve neutrophils were cultured in fresh media and left untreated (UT) or stimulated with $F$. alocis, or they were cultured in the conditioned media from untreated cells or cells stimulated with $F$. alocis after pre-treatment with media, neutralizing TLR2/6 antibodies or isotype controls. After culture for 24 hours, the cells were stained for Annexin V and 7AAD. Data are plotted as the mean percent viable (Q4: Annexin V-, 7AAD-) neutrophils \pm SEM from five independent experiments. One-way ANOVAs with Tukey post-hoc testing was performed on $\mathrm{C} \& \mathrm{D}$ to determine statistical significance. $*=\mathrm{p}>0.05, * *=\mathrm{p}>$ $0.01, * * *=p>0.001$ 
A.
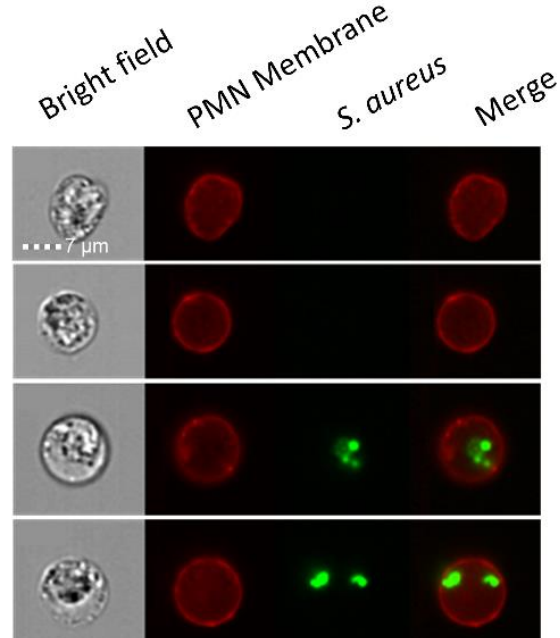

C.

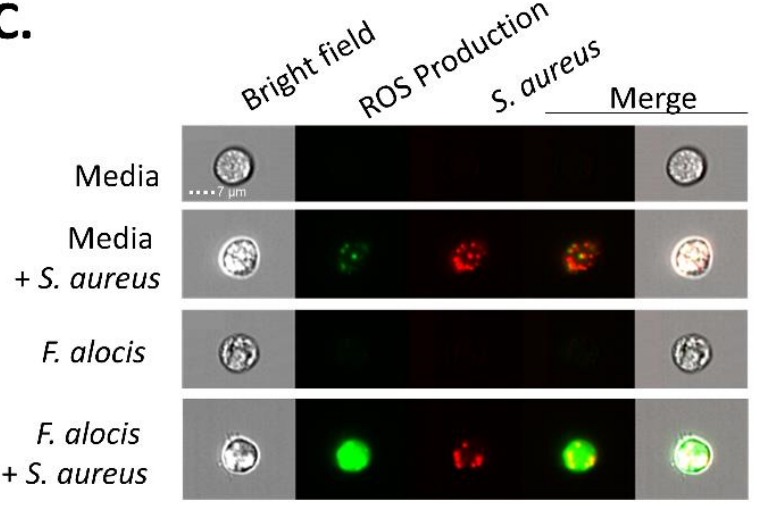

B.

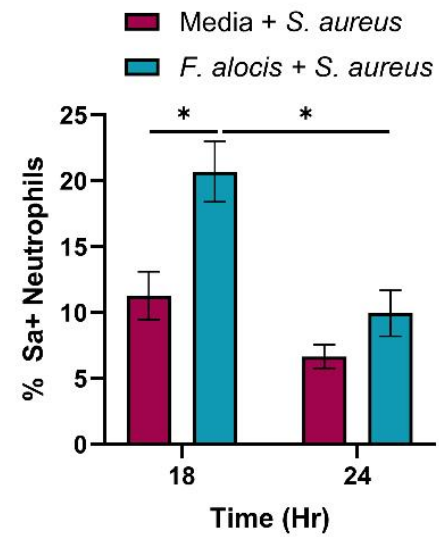

D.

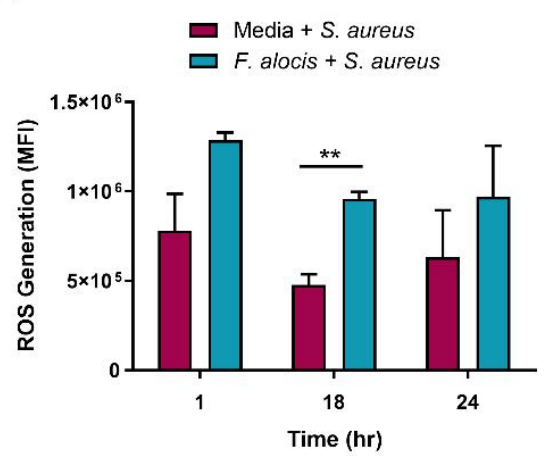

Figure 4.11: $F$. alocis-challenged neutrophils retain functional capacity for longer timepoints. 
Figure 4.11: Neutrophils were aged for 18 or 24 hours in media or stimulation with $F$. alocis followed by a 30 -minute challenge with $S$. aureus. First, the phagocytic capacity of these cells was tested via imaging flow cytometer. (A) Images from the imaging flow cytometer depict neutrophils that did or did not internalize S. aureus. (B) Summary from 3 independent experiments shows the mean percentage of neutrophils that phagocytosed $S$. aureus. (C) Representative images from the imaging flow cytometer show the ROS production in response to $S$. aureus challenge by cells aged for 18 hours with media or $F$. alocis. (D) Represents the average mean fluorescence intensity \pm SEM from media-aged cells or F. alocis-aged cells that internalized $S$. aureus from 3 independent experiments. Two-way ANOVAs with Bonferroni post-hoc testing was performed on B \& D to determine statistical significance. $*=p>0.05, * *=p>0.01, * * *=p>0.001$. 


\section{CHAPTER 5:}

\section{THE FINAL FRONTIER: CLEARANCE OF NEUTROPHILS AFTER $F$. ALOCIS CHALLENGE}

\section{Introduction:}

After the activation of a local inflammatory response due to infection, the proper termination of the inflammatory cascade is important because it initiates a phase of tissue repair and resolution responses. Improper resolution of inflammation results in excessive scarring, organ damage, chronic inflammation, and loss of self-tolerance, which contributes to multiple pathologies like cardiovascular disease, auto-immunity, and cancer [442-444]. An important initiating step in this pathway is the clearing of dead or spent cells through a process called efferocytosis.

In vivo, efferocytosis of apoptotic cell corpses occurs extremely quickly; for example, when six million apoptotic cells are injected into the peritoneum of mice, the cells are taken up by phagocytes within 15-30 min [445]. However, in a number of nonresolving, chronic inflammatory diseases, defects in efferocytosis develop and result in an accumulation of dead cells [443]. When cells undergo apoptosis, they undergo a noninflammatory cell death because the cells maintain their membrane integrity for a limited period of time. Nevertheless, they need to be cleared quickly to prevent secondary necrosis, which will release their cytotoxic molecules into the extracellular space. 
In order to orchestrate efferocytosis, four main steps must take place. First, chemotactic "find-me" signals are produced by dying neutrophils to attract efferocytic cells. These signals include the nucleotides ATP and UTP, the chemokine fractalkine (CX3CL1), and the lipids lysophosphatidylcholine (LPC) and sphingosine-1-phosphate (S1P) [446]. Next, "eat-me" signals on the surface of the apoptotic cell mediate recognition by macrophages. To distinguish them from viable cells, apoptotic cells downregulate expression of "don't eat me" signals like CD47, CD61, CD46 and CD31 and upregulate expression of lipids (phosphatidylserine), proteins (ICAM-3), or modified carbohydrates [442]. The third step involves the uptake and degradation of the apoptotic body in the phagolysosomal compartment by proteases, DNAses and lipases. Finally, efferocytic phagocytes release a number of anti-inflammatory mediators including PGE2, TGF- $\beta$, IL10, and lactate, among others to initiate the resolution of inflammation [445].

In the context of periodontitis, not much was known about how periodontal pathogens affect efferocytosis. However, pervasive defects in efferocytosis could be contributing to the progression of periodontitis and the chronicity of inflammation in the oral cavity. In chapter 2, I observed that $F$. alocis challenge induces significant differential expression of pathways related to apoptosis and in chapter 4 our results demonstrated that F. alocis prolongs the functional lifespan of neutrophils. Therefore, I hypothesized that $F$. alocis interferes with efferocytosis to inhibit resolution of inflammation. Paradoxically, neutrophils that were cultured with $F$. alocis were efferocytosed with greater frequency than untreated neutrophils. However, once internalized, the F. alocis-challenged neutrophils were not degraded as quickly as efferocytosed untreated neutrophils. 
Ultimately, I believe that under the right conditions, F. alocis could be using neutrophils as "trojan horses" to gain access into a longer-lived host cell.

\section{Materials \& Methods:}

Neutrophil isolation: Human donor recruitment, blood draws, and materials required for this procedure were in accordance with the guidelines approved by the Institutional Review Board of the University of Louisville. Neutrophils were isolated from venous blood of healthy donors using plasma-Percoll gradients, as described previously [297].

Bacterial strains and growth conditions: F. alocis ATCC 38596 was cultured in brain heart infusion (BHI) broth supplemented $5 \mathrm{mg} / \mathrm{mL}$ yeast extract, L-cysteine $(0.05 \%)$ and arginine $(0.05 \%)$ for 7 days anaerobically at $37{ }^{\circ} \mathrm{C}$ as previously described $[\mathbf{2 7 5}, \mathbf{2 7 6}$. Serum opsonization was performed by incubating $F$. alocis at $37^{\circ} \mathrm{C}$ for 20 min in $10 \%$ normal human serum (Complement Technology, Inc., Tyler, TX, USA). Heat killed F. alocis was generated by incubation at $90{ }^{\circ} \mathrm{C}$ for $60 \mathrm{~min}$. Non-viability was confirmed by incubation in culture media at same conditions used for the live organism. After opsonization, $F$. alocis was labeled with carboxyfluorescein succinimidyl ester (CFSE; 40 $\mathrm{ng} / \mu \mathrm{l}$ ) for $30 \mathrm{~min}$ at room temperature in the dark and washed 3 times with PBS prior to use.

Aging and staining of neutrophils: The membranes of neutrophils were stained with PKH26 using the manufacturer's protocol (Sigma). After several washes with PBS to remove excess dye, neutrophils were cultured in clear RPMI-1640 with L-glutamine and 5\% heat 
inactivated human serum with or without opsonized F. alocis (MOI 10) at $37{ }^{\circ} \mathrm{C}, 5 \% \mathrm{CO}_{2}$ for 18 hours. After 18 hours of culture neutrophils were stained for CD47 and CD31 (Biolegend, Carlsbad, CA, USA). Aged neutrophils were washed before adding to cultured macrophages.

Mice and peritoneal macrophage extraction: All experiments were completed on male and female C57B1/6 wild-type mice between 8 to 12 weeks of age. The institutional animal care and use committees at University of Louisville approved all animal experiments. As previously described, mice were injected with $5 \mathrm{mM}$ sodium periodate, sacrificed after 72 to 96 hours, and peritoneal cavities lavaged with phosphate-buffered saline containing 2 mM EDTA $[447,448]$. Total lavage cells were plated in tissue-culture plates or chamber slides in Iscove modified Dulbecco medium with $10 \%$ heat-inactivated fetal bovine serum for 2 hours. Nonadherent cells were removed, and adherent macrophages cultured overnight before use in experiments.

Human Macrophage culture: The mononuclear cell layer fraction from peripheral blood of healthy donors was washed thrice using Krebs buffer. Then, the cells were plated in 6 well plates at density of 2 million cells per well in krebs buffer containing 2mM HEPES and allow to settle for 2 hours. The unsettled cells were removed and settled cells were washed with krebs buffer. Next, the settled cells were gently scraped, plated on 6 well low attachment plate in RPMI with $20 \% \mathrm{FBS}$, and incubated at $37{ }^{\circ} \mathrm{C}$ in a $5 \% \mathrm{CO}_{2}$ incubator. On the fourth day, the number of big cells was counted followed by plating the cells at the density of 0.2 million cells on coverslips in 24 well in RPMI with 10\% FBS. The media 
was changed in the wells on day sixth to RPMI with 5\% FBS and on day seventh to RPMI with 1\% FBS. The human monocytes derived macrophages were used for experiment on eighth day, when $60 \%$ or more of the cells were expressing CD68, a marker for differentiated macrophages.

Macrophage phagocytosis assays: Macrophages that were seeded overnight onto on 8-well glass chamber slides or 12 well plates were pulse fed (centrifuged $1 \mathrm{~min}, 800 \mathrm{~g}, 25^{\circ} \mathrm{C}$ ) neutrophils incubated in media for 18 hours or opsonized $F$. alocis-cultured neutrophils at a MOI of 10 neutrophils per macrophage for 30 or 60 minutes at $37^{\circ} \mathrm{C}$. The slides or wells were then washed 3 times with sterile PBS, which removed any uningested neutrophils.

For myeloperoxidase (MPO) staining, the 8-well chamber slides were fixed using 4\% paraformaldehyde. Then, macrophages were stained for MPO by adding 3,3'diaminobenzidine (DAB) as previously shown [447, 448]. 100 cells were counted for each experimental condition and qualified based on whether they were positive for MPO from phagocytized neutrophils.

For flow cytometry experiments, antibodies for F4/80 (Mouse, BioLegend, \#123118) and CD16 (Human, BioLegend, \#302012) were added to the wells to stain the cells on ice for 20 minutes. Cells were then scraped and 20,000 cells were collected on a on a BD FACSCelesta flow cytometer and analyzed using the FlowJo software (Ashland, OR, USA). To obtain the \% ingestion figure, the percentage of F4/80+, CD16+ cells (which represents macrophages with attached neutrophils) was subtracted from the percentage of F4/80+, PKH-26+ cells. 
For confocal imaging, macrophages were seeded overnight onto on 8-well glass chamber slides and then fed aged PKH-26-labeled neutrophils that had been challenged with CFSE-labeled, opsonized F. alocis. After feeding for an hour, the neutrophils were washed off and the macrophages were processed for confocal staining or left for another 23 hours of culture. Upon completion of each timepoint, the cells were stained with antibodies against F4/80. The cells were fixed with $4 \%$ paraformaldehyde and imaged by confocal microscopy to assess whether the neutrophils and/or F. alocis ingested by the macrophages had been degraded.

Macrophage Supernatant and Lysate Collection: Macrophages that were seeded overnight on 12 well plates in duplicates were left untreated or fed neutrophils for an hour as previously described. The washes to remove uningested neutrophils were pooled, pelleted and lysed on ice for 30 minutes in the lysis buffer described above. Fresh, warmed media was added to the wells with macrophages, and the cells were incubated for an additional hour (for total of 2 hours with PMN) or 23 hours (for a total of 24 hours with PMN). At each indicated timepoint, $25 \mu \mathrm{L}$ of lysis buffer was added to each well. Then, the cells were scraped into the lysis buffer, the duplicates were combined and placed on ice for 30 minutes. The lysates and their respective western blots were generated as described for neutrophils above, with the exception that $20 \mu \mathrm{g}$ of the cell lysates were separated by $12 \%$ SDS-PAGE and immunoblotted with antibodies for F. alocis (1:2000, generated by Pocono using UV killed F. alocis), elastase (1 $\mu \mathrm{g} / \mathrm{ml}$, Abcam), or $\beta$-actin (1:1000, Cell Signaling). Additionally, at the completion of the 24-hour timepoint, the supernatants were saved from the wells, centrifuged to remove any cell debris and $1 \%$ protease and phosphatase inhibitors 
were added to the supernatants to protect them from degradation. These supernatants were run on a 32-analyte multiplex assay to determine the cytokine profile.

F. alocis Colony Forming Units: For each timepoint tested, three culture tubes were generated. The first one contained the F. alocis inoculum in the neutrophil culture media, the other two tubes had the $F$. alocis inoculum co-cultured with human neutrophils. At the end of each timepoint, the $F$. alocis inoculum tube and one of the tubes with F. alocis and neutrophils were placed on ice while the second tube containing $F$. alocis and neutrophils was centrifuged $\left(300 \mathrm{~g}, 5 \mathrm{~min}, 4^{\circ} \mathrm{C}\right)$ and washed 3 times to remove extracellular bacteria. During the final wash of the intracellular bacteria condition, the F. alocis inoculum tube and the tube with the total $F$. alocis and neutrophils were centrifuged $\left(6000 \mathrm{~g}, 2 \mathrm{~min}, 4{ }^{\circ} \mathrm{C}\right)$ to pellet all bacteria and immune cells. The pellets from the inoculum, the total F. alocis and neutrophils, and the intracellular $F$. alocis were lysed for 1 minute in $0.02 \%$ saponin and serially diluted in sterile PBS. These dilutions were sterilely plated in duplicate $10 \mu \mathrm{L}$ drop aliquots on anaerobic BHI plates (BHI media (7.4g/200ml), L-cysteine $(0.1 \mathrm{~g} / 200 \mathrm{ml})$, yeast extract $(1 \mathrm{~g} / 200 \mathrm{ml})$, agar powder $(3 \mathrm{~g} / 200 \mathrm{ml}), 0.05 \%$ arginine and $5 \%$ sheep blood). The plates were transferred into an anaerobic chamber and allowed to grow for 72 hours. Colonies that grew were counted and Gram stained. In experiments where anaerobic and aerobic conditions were tested in parallel, the anaerobic neutrophil media was put into the anaerobic chamber and the aerobic media was left in an aerobic incubator the night before performing the assay. 
Statistical Analysis: Statistical differences among experimental conditions and time points were analyzed using GraphPad Prism Software (Graphpad San Diego, CA, USA). Differences were considered significant at the level $\mathrm{P}<0.05$. The specific statistical test for each experiment is listed in their respective figure legend. If results represent data from multiple experiments, mean values \pm standard error of mean (SEM) are shown.

\section{Results:}

\section{Efferocytosis of neutrophils aged with $\mathrm{F}$. alocis}

Since $F$. alocis-challenged neutrophils have extended survival and retain their functional capabilities, I hypothesized that efferocytosis of these $F$. alocis neutrophils would be hampered. To test this, I acquired mouse macrophages by inducing sterile peritonitis in wild-type mice. These peritoneal macrophages were pulse fed for 30-60 minutes with 18 hours aged human neutrophils that were either untreated (media) or challenged with F. alocis (MOI; 10 bacteria per neutrophil); the feeding ratio used was of 10 neutrophils per macrophage. After 30 or 60 minutes, uningested neutrophils were washed off and the macrophages were stained for myeloperoxidase (MPO) as a way to visualize internalized neutrophils, which can be appreciated in the representative images (Figure 5.1A). 100 macrophages from each condition were tallied based on whether they ingested neutrophils, and at both 30 and 60 minutes, neutrophils challenged with $F$. alocis were efferocytosed by more macrophages compared to the untreated neutrophils (Figure 5.1B). Ultimately, I chose to use the mouse macrophage/human neutrophil model for the rest of the efferocytosis experiments; however, I wanted to determine if the pattern of ingestion was the same in human monocyte derived macrophages (HMDM) (Figure 5.1C). 
Only one replicate with one donor was conducted, but in HMDM, F. alocis-stimulated neutrophils were also efferocytosed more readily at both timepoints tested.

To confirm the MPO imaging data, I used flow cytometry as a high-throughput technique to evaluate the internalization of $\mathrm{PKH}-26$ labeled neutrophils by F4/80 expressing macrophages. Figure 5.1D depicts representative flow cytometry dot plots that show the gating strategy employed to determine uptake. The untreated macrophage control only stained positive for F4/80, but when macrophages were fed untreated neutrophils, there was a shift to the right, indicating neutrophil uptake. This shift was even more pronounced in macrophages that were fed F. alocis-stimulated neutrophils (Figure 5.1D). When the population of macrophages that ingested neutrophils was combined from several mice, $\sim 20 \%$ of macrophages engulfed untreated neutrophils after 30 minutes and this value doubled when the ingestion time was increased to 60 minutes (Figure 5.1E). In the case of F. alocis-stimulated neutrophils, $40 \%$ of macrophages had ingested neutrophils at $30 \mathrm{~min}$, and at 60 minutes this value rose to $80 \%$ of macrophages. Thus, the enhanced ingestion of F. alocis-treated neutrophils observed by microscopy was also mirrored in the more robust flow cytometry approach (Figure 5.1E).

\section{Expression of "Don't eat me" signals on F. alocis-stimulated neutrophils}

The best characterized "eat me" signal on apoptotic cells is the expression of phosphatidyl serine (PS) on the cell surface. However, F. alocis stimulation results in decreased externalization of PS as compared to unstimulated cells (Chapter 4). Therefore, I hypothesized that instead of expressing more "eat me" signals than untreated controls, perhaps F. alocis neutrophils express fewer “don't eat me” signals like CD47 (Figure 5.2A) 
and CD31 (Figure 5.2B). Both CD47 and CD31 are shed naturally as neutrophils age, which is why there is a decrease in the expression of both of these markers between freshly isolated neutrophils and neutrophils aged for 18 hours. Concomitantly, stimulating the neutrophils with pro-apoptotic Fas L resulted in decreased expression of both CD47 and CD31 as compared to neutrophils cultured in media for 18 hours. In the case of CD47, $F$. alocis-stimulated cells expressed significantly more CD47 than untreated cells (Figure 5.2A). On the other hand, F. alocis stimulated cells expressed significantly less CD31 than media-cultured cells (Figure5.2B). In fact, the expression of CD31 on F. alocis stimulated neutrophils was comparable to that of the pro-apoptotic stimulant, Fas L.

Previous reports have shown that the secreted proteases of oral bacteria like $P$. gingivalis can cleave CD31 to induce the efferocytosis of viable cells [449, 450]. To assess if viable $F$. alocis is producing a protease that is cleaving of CD31 on viable cells, neutrophils were stimulated with heat-killed $F$. alocis. In terms of CD47 expression, both heat-killed and viable $F$. alocis resulted in comparable CD47 expression (Figure 5.2A). However, although it did not reach significance, treating the neutrophils with HK F. alocis resulted in a partial rescue of the CD31 expression (Figure 5.2B). Together, this suggests that the decreased expression of CD31 may be due to released $F$. alocis proteases. Additionally, neutrophils can release their own proteases through granule exocytosis that can also cleave off neutrophil membrane receptors [449]. To confirm the decreased CD31 expression was due to the action of proteases, I cultured neutrophils with media or F. alocis in the presence of protease inhibitors (PI) and then quantified the expression of CD47 and CD31 (Figure 5.2C-D). The presence of PI restored CD47 expression to fresh cells levels for both untreated and $F$. alocis-stimulated neutrophils, indicating that proteases are part 
of the cells normal CD47 downregulation, regardless of infection (Figure 5.2D). However, in the case of CD31, the inhibitors did not restore CD31 of any of the conditions to the level of fresh cells (Figure 5.2D). In media-cultured cells, the expression of CD31 was the same whether or not the inhibitors were present. In $F$. alocis stimulated cells, protease inhibitors restored CD31 levels to the media-cultured cells, suggesting that in F. alocis infection, proteases are responsible for the added shedding of CD31. Taken together, this data suggests that macrophages are able to engulf viable, $F$. alocis-challenged cells more than apoptotic untreated cells because $F$. alocis causes a decrease in expression of CD31 through proteases.

\section{Macrophage degradation of $\mathrm{F}$. alocis-cultured neutrophils}

After recognizing dead cells, the efferocytic macrophages begin to digest the phagocytized cargo. In our system, I examined the digestion of neutrophils by confocal microscopy (Figure 5.3A-B). After feeding membrane-labeled neutrophils for an hour, the uningested granulocytes were washed off and the macrophages were either fixed for imaging or left to digest their neutrophil cargo for an additional 23 hours. Consistent with our previous results, after 1 hour of feeding, F. alocis-neutrophils were ingested more than media-cultured cells (Figure 5.1A). Notably, CFSE-labeled F. alocis could still be detected within neutrophils that were efferocytosed. Then, after 24 hours of culture between the neutrophils and the macrophages, the ingested media-cultured neutrophils had been almost entirely degraded whereas the $F$. alocis-stimulated neutrophils remained more intact, with F. alocis still detectable intracellularly. Figure 5.3B shows a zoomed portion of the merged 
images shown in panel A, to better appreciate the architecture of the neutrophils within the macrophages.

To quantify this phenomenon, the same experiments were conducted, but this time, instead of viewing the cells on the confocal microscope, whole cell extracts were collected and western blots of these lysates were probed with polyclonal antibodies against $F$. alocis as a measure of bacterial proliferation or probed for elastase as a measure of neutrophil presence within the macrophage (Figure 5.4A). On the representative western blot shown, the first thing to appreciate is that the $F$. alocis antibody recognizes proteins from neutrophils (UT PMN) and macrophages (Mo) even when the bacterium is not present. Thus, quantification of these blots was done on the most prominent $F$. alocis protein band that could be detected, which is the one delineated by the blue box. Quantification that focused on this band showed an incremental decrease, but not a complete degradation, of F. alocis proteins within macrophages (Figure 5.4B). Next, the blots were probed for elastase as a measure of neutrophil presence. The first two lanes, which represent the lysates of neutrophils washed off the macrophages that were not ingested, the amount of elastase was less in the F. alocis-stimulated neutrophils, suggesting that more neutrophils were ingested by the macrophages, as has been shown previously. Unlike the elastase from untreated neutrophils inside the macrophages, the elastase from $F$. alocis-stimulated neutrophils could still be detected after 24 hours of culture, indicating that the efferosome is not as efficient at degrading F. alocis-stimulated neutrophils (Figure 5.4C).

\section{Neutrophils as "trojan horses" for F. alocis}


While some pathogens delay neutrophil death to maintain viability of their replicative niche and others will accelerate apoptosis or trigger PMN lysis to evade intracellular killing. Another proposed mechanism is the use of infected neutrophils as "Trojan horses" for infection of macrophages. Since F. alocis extends neutrophil lifespan and delays the degradation of neutrophils inside macrophages, I tested the possibility that F. alocis was using neutrophils to create another niche inside the longer-lived macrophages.

First, I assessed by flow cytometry how many macrophages internalized neutrophils that contained $F$. alocis to see if macrophages preferentially engulfed cells harboring bacteria. To accomplish this, $\mathrm{PKH}-26$ labeled neutrophils were co-cultured with CFSE-labeled $F$. alocis for 18 hours followed by feeding of macrophages for 1 hour. Using the same gating strategy as Figure 5.1D, the population of macrophages that efferocytosed neutrophils was determined (Figure 5.5A). These PKH-26 positive macrophages (Q2: $\mathrm{F} 4 / 80+, \mathrm{PKH}+$ ) were further characterized based on their intensity of CFSE as a measure of the percent of neutrophil+ macrophages that also contained $F$. alocis (Figure 5.5A). The summary figure of these experiments shows that as expected, $100 \%$ of the macrophages that phagocytized media-cultured neutrophils did not contain any F. alocis (CFSE negative (-)). However, when macrophages were fed F. alocis-stimulated neutrophils, $50 \%$ of the macrophages internalized $F$. alocis-containing neutrophils (Figure 5.5B). In the condition where macrophaes were fed $F$. alocis-stimulated neutrophils, there was a small $(5-10 \%)$ difference between the CFSE(-) and the CFSE (+) macrophages; however, despite reaching statistical significance, is likely not biologically relevant. Together, this data demonstrates that macrophages are not preferentially efferocytosing $F$. alocis infected neutrophils. 
The basis behind cellular "trojan horses" is that a viable microbe drives the uptake of their cell host by another cell that will provide a longer-lived or more permissive replicative niche. However, the viability of $F$. alocis in culture with neutrophils had only been tested up to 4 hours [284]. Therefore, the survival of $F$. alocis with neutrophils was tested for longer timepoints (Figure 5.5C). At 1 and 4 hours of culture, neutrophils were responsible for an overall reduction of viable $F$. alocis as compared to the inoculum alone. However, at 10 hours both the inoculum and the neutrophil-cultured F. alocis were markedly affected in their CFU counts. Parallel to these experiments, extracellular F. alocis was extensively washed off and intracellular F. alocis was plated (Figure 5.5D). Data shows that neutrophils do not reduce $F$. alocis numbers within the first 4 hours of challenge. However, by 10 hours of culture the quantity of CFU dramatically decreased, same as the inoculum and the total culture of $F$. alocis with neutrophils. Since $F$. alocis is a strict anaerobe, I hypothesized that by 10 hours, the aerobic environment was inactivating the oral pathogen.

Due to their limited oxygen diffusion capacity, normal tissues are considered physiologically hypoxic [451]. Tissues can become increasingly hypoxic during infection through the activation of the NADPH oxidase complex [452], and as the depth of the periodontal pocket increases, the oxygen tension also decreases [453]. Neutrophils are likely exposed to profound levels of hypoxia as they are largely recruited to periodontal disease-associated tissues or trapped in areas of microcirculatory impairment. As a crude method to mimic the environment where neutrophils are exposed to F. alocis in vivo, neutrophils $\pm F$. alocis were cultured in anaerobic media in an anaerobic chamber or in aerobic media in an incubator. First, the apoptotic status of these neutrophils was tested 
after 24 hours (Figure 5.5E). Culturing neutrophils anaerobically results in enhanced neutrophil viability in untreated cells, but in both sets of cells, F. alocis extended the leukocytes' survival even further.

Then, the ability of these cells to kill F. alocis was tested (Figure 5.5F). At 1 and 4 hours of culture, there was no difference in the killing efficiency of neutrophils cultured aerobically or anaerobically. After 10 hours and overnight incubations, F. alocis was eliminated in aerobic culture conditions as previously shown. Interestingly, under anaerobic conditions, only the $F$. alocis cultured with neutrophils at both of these timepoints produced CFUs, indicating that $F$. alocis lifespan is extended in the presence of neutrophils. F. alocis did not proliferate more than the inoculum at any timepoint tested. When the intracellular bacteria were plated, aerobically cultured bacteria perished by 10 hours, but anaerobically cultured bacteria could form colonies even after overnight culture with neutrophils (Figure 5.5G). There was also no significant difference between the CFUs counts at 1, 4, and 10 hours, indicating that under anaerobic conditions, F. alocis is able to dampen neutrophil microbicidal mechanisms for long periods of time. Taken together, this data shows that under anaerobic conditions, $F$. alocis requires the presence of neutrophils to survive for extended periods of time, and also that the bacterium is able to remain viable within neutrophils for long periods of time. These findings are particularly exciting because they could mean that under hypoxic conditions, $F$. alocis could be using neutrophils as vehicles to enter macrophages as another niche.

Efferocytosis of F. alocis-cultured neutrophils induces a pro-inflammatory environment 
The final step in efferocytosis is the release of anti-inflammatory mediators that will tip the scale towards the resolution of inflammation. To determine if this was the case, I collected supernatants for cytokine analysis from untreated macrophages or macrophages that efferocytosed untreated neutrophils or neutrophils exposed to $F$. alocis. The cytokine multiplex data showed that efferocytosing $F$. alocis-neutrophils results in macrophages releasing a significant amount of pro-inflammatory cytokines like G-CSF, IL-1 $\alpha$, IL$1 \beta$, TNF $\alpha$, and IL-6 (Figure 5.6). There was also an upward trend in the release of the proinflammatory cytokine IFN $\gamma$ but this did not reach significance. Furthermore, the generation of chemokines like RANTES (CCL5), KC (CXCL1), IP-10 (CXCL10), LIX (CXCL5), MCP1 (CCL2), and MIP2 (CXCL2) was also amplified compared to macrophages alone or macrophages that received untreated neutrophils (Figure 5.7). Paradoxically, macrophages that internalized F. alocis-stimulated-neutrophils produced significant amounts of the anti-inflammatory cytokine, IL-10. Other cytokines like IL-17, MIG (CXCL9), MIP-1 $\alpha$ (CCL3), and MIP-1 $\beta$ (CCL4), were not significant. Overall, the cytokine data shown here suggests that the resolution function of efferocytosis is corrupted when the macrophages ingest $F$. alocis challenged neutrophils. These efferocytosing cells will produce pro-inflammatory cytokines and chemokines that will recruit more immune cells. Together, this response will feed into the dysregulated chronic inflammation cycle of periodontal disease and prevent the resolution.

\section{$\underline{\text { Discussion }}$}


Since challenge with $F$. alocis delayed the externalization of phosphatidyl serine (PS), and the cells retained their functional capacity after 18 hours of challenge, I hypothesized that efferocytosis would also be defective. Surprisingly, this was not the case, since $F$. alocis challenged neutrophils were efferocytosed by macrophages significantly more than control cells. It has been previously demonstrated that PS expression is not absolutely necessary for the uptake of neutrophils by macrophages [449]. Thus, when the expression of "don't eat me" signals was tested, F. alocis-neutrophils showed enhanced membrane expression of CD47 but decreased expression of CD31. CD47 on viable neutrophil membranes will be recognized by the macrophage receptor SIRP $\alpha$ (signal regulatory protein $\alpha$ ) that activates inhibitory phosphatases (SHP-1 and SHP-2) via ITIMs (immunoreceptor tyrosine-based inhibition motifs) in SIRP $\alpha$. Overall, this results in the inhibition of macrophage efferocytosis [454]. CD31 expression prevents phagocyte ingestion of viable cells by transmitting 'detachment' signals to the macrophage [455]. It is possible that despite the high expression of CD47 in F. alocis-neutrophils, this is not enough to overcome the lack of repulsive signaling from the diminished expression of CD31. In fact, when CD31 was cleaved off of freshly isolated neutrophils by $P$. gingivalis gingipains, the neutrophils were efficiently engulfed by macrophages [449].

In our system, HK $F$. alocis induced a milder reduction in CD31, suggesting that like $P$. gingivalis, viable $F$. alocis is actively inducing the proteolytic cleavage of CD31. It will be interesting to test whether HK F. alocis-stimulated neutrophils are efferocytosed to similar levels as untreated cells, since they display comparable levels of CD31. Other proteinase activity sources that should be considered are elastase and cathepsin G, two neutrophil-derived proteases typically found in azurophilic granules and on the surface of 
neutrophils when they are primed [456]. Previous results from our laboratory show that azurophilic granules are not released at early timepoints (30 $\mathrm{min})$ [284], but the release of azurophilic granule content during longer challenge periods has not been determined. Importantly, both of these proteases have been linked to modifications that modulate efferocytosis. Elastase cleaves the phosphatidyl serine receptor on phagocytes, leading to defective apoptotic cell clearance [457] and cathepsin G cleaves CD31 on neutrophils to hinder uptake by macrophages [449]. Our studies used a pan-protease inhibitor cocktail, but it may be valuable to use specific inhibitors for each of the proteases to determine if they play a role in the $\mathrm{CD} 31$ shedding.

In our experimental set up, $F$. alocis was cultured aerobically with neutrophils, meaning that by 10 hours most of the strictly anaerobic bacteria was no longer viable. Regardless of this, bacteria could still be visualized within neutrophils that were engulfed by macrophages up to 24 hours after efferocytosis. This brings up several important questions that revolve around the possibility of neutrophils as "trojan horses" for the infection of macrophages. After culture of neutrophils with $F$. alocis, how early can the neutrophils be internalized by efferocytes? If $F$. alocis is viable within the apoptotic cells, can the bacterium survive and/or proliferate within the apoptotic cell phagosome?

As a crude way to determine the role of atmospheric oxygen on the neutrophil- $F$. alocis interaction, I performed the infection under aerobic or anaerobic conditions. The basal length of neutrophil lifespan was prolonged, but $F$. alocis challenge increased the number of viable neutrophils even further. Of course, longer timepoints and more precise environmental oxygen controls will be necessary to assess the full impact of $F$. alocismediated survival under hypoxic conditions. It was not surprising that anaerobic conditions 
prolonged the survival of $F$. alocis, but it was unexpected that in the 10 hour and overnight timepoints, only the $F$. alocis conditions that received neutrophils had CFU formation, suggesting that the bacteria are able to survive better in the presence of neutrophil-driven inflammation. Intracellular CFU could be detected in the overnight cultures, but since these assays were done in suspension it is not possible to guarantee the intracellular bacteria detected were present in neutrophils the entire culture period. While it will need to be tested in our system, I believe that the decreased bacterial killing under anaerobic conditions cannot be attributed to defects in neutrophil functions because of decreased availability of oxygen. Studies that have specifically tested neutrophil functionality describe that hypoxia does not affect phagocytosis, but enhances neutrophil degranulation, the autophagic capacity of neutrophils, and the killing of some microbes like Escherichia coli and $S$. aureus $[451,458]$. The only function that was impaired seemed to be the production of ROS, but in the case of $F$. alocis, the bacterium is able to grow better in the presence of hydrogen peroxide and at least aerobically, induces minimal ROS generation from neutrophils [58, 284].

The fate of the pathogen and the immune cell in which it resides in will highly depend on the type of efferocyte, host factors like genetic polymorphisms, and the type of cell death the immune cell containing the bacterium underwent [443]. For example, low virulence strains of Mycobacterium tuberculosis induce apoptosis in cells and when macrophages engulf these cells, the lysosomal fusion with the apoptotic cell phagosomes promotes bacterial killing [459]. However, virulent M. tuberculosis strains induce necrosis in neutrophils and when the necrotic neutrophils are internalized by macrophages, $M$. tuberculosis intracellular growth is promoted [460]. Thus, it is possible that despite 
efficiently engulfing $F$. alocis-challenged neutrophils, macrophages are not able to digest the cargo because the viable neutrophils did not trigger the signaling needed for full phagosome maturation and subsequent degradative capacity. To prove this, more studies are needed to characterize the macrophage phagosome that contains $F$. alocis-stimulated neutrophils. One of the first things to test would be ROS production and phagosome acidification, since they are essential for efficient proteolysis of apoptotic cells within efferosomes [447].

Instead of initiating a pro-resolution phenotype, the macrophages that ingested the F. alocis-laden neutrophils secrete a number of pro-inflammatory cytokines and chemokines that will likely contribute to drive the chronicity of periodontal inflammation. Another indirect consequence of defective efferocytosis is the development of autoimmune responses. Normally, efferocytosis helps mount an adaptive immune response against bacteria through the process of antigen cross-presentation [443]. However, when there is limited proteolysis of phagosomal cargo, there is enhanced presentation of antigens and increased potential for excessive activation of adaptive immune responses [443, 447]. To address this point, it will be interesting to see if the cross-presentation is different between macrophages that ingested untreated or F. alocis stimulated neutrophils.

Efferocytosis in the oral cavity has also been ill-defined, especially when it comes to the contribution of emerging oral pathogens. Despite being functional and viable, neutrophils that interact with $F$. alocis will be efficiently ingested by efferocytic macrophages. However, instead of initiating the resolution of inflammation, the macrophages will add to the pro-inflammatory milieu by secreting more cytokines and chemokines. Ultimately, these result begin to delineate how engulfing $F$. alocis-stimulated 
neutrophils can influence the action of efferocytic macrophages and how F. alocis may be contributing to the chronic dysregulated inflammation observed in periodontitis. 

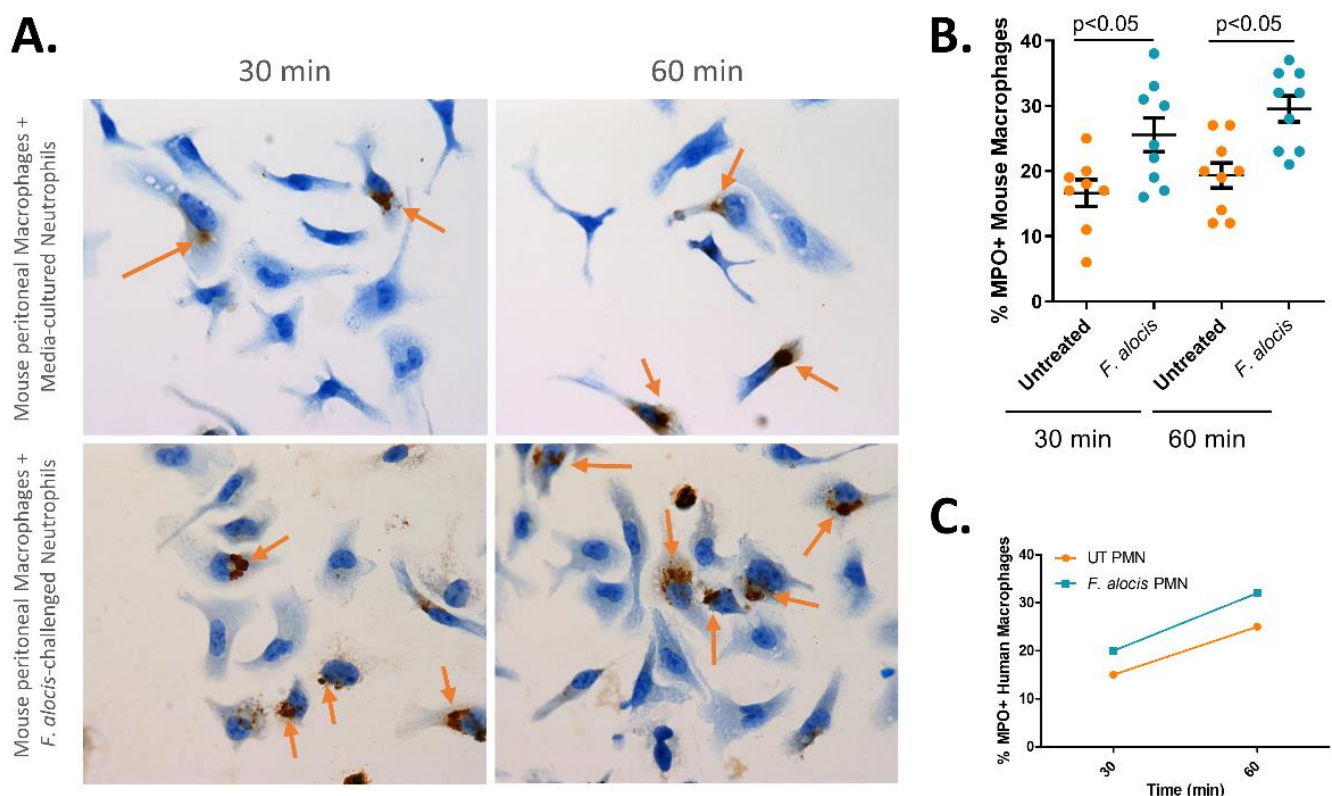

D.

Mouse peritoneal Macrophages + $18 \mathrm{H}$ Untreated Neutrophils

C.
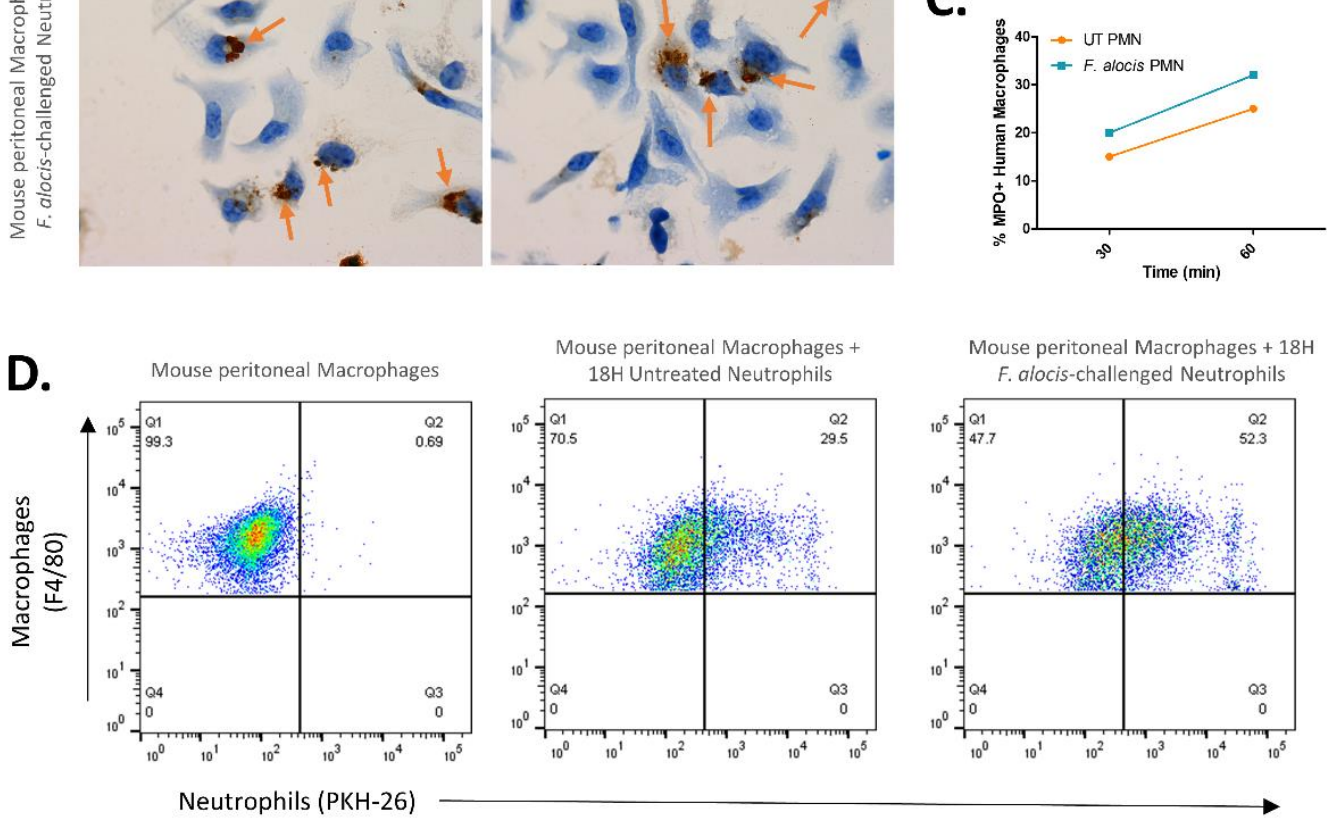

Mouse peritoneal Macrophages $+18 \mathrm{H}$ F. alocis-challenged Neutrophils

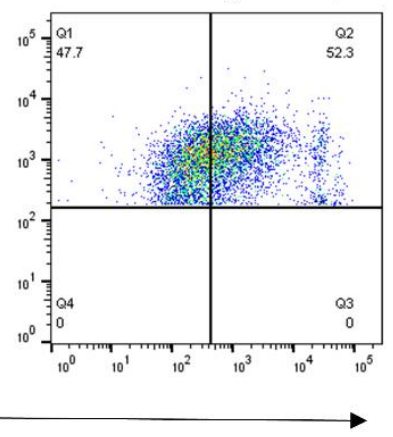

E.

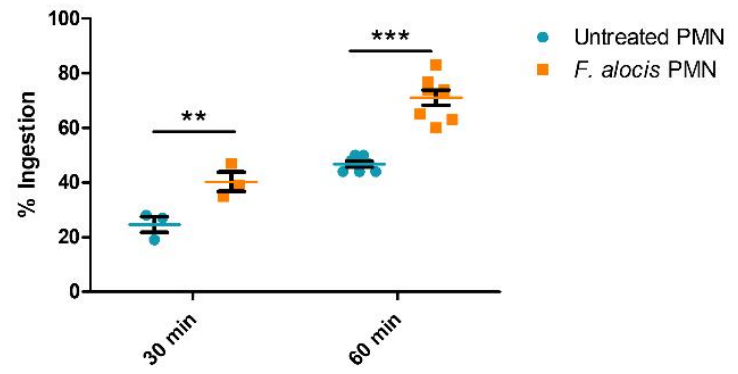

Figure 5.1: Efferocytosis of $\boldsymbol{F}$. alocis-challenged neutrophils is more efficient than media-cultured neutrophils. 
Figure 5.1: Neutrophils were aged for 18 hours in media or with $F$. alocis followed by a 30- or 60-minute pulse feeding of macrophages. (A) Representative images show MPO staining of mouse peritoneal macrophages after neutrophil feeding. Arrows indicate engulfed neutrophils. (B) 100 macrophages from each condition were qualified based on whether they had efferocytosed neutrophils. The percentage of myeloperoxidase (MPO) positive macrophages were plotted \pm SEM where each dot represents the macrophages from one mouse, conducted over 3 experimental replicates. A two-tailed student T-test was performed to determine statistical significance at each timepoint. (C) The efferocytosis assay and MPO staining was completed for one replicate using human neutrophils and human macrophages. Data are plotted as in B. (D) Flow cytometry dot plots show the gating strategy to determine the uptake of PKH-26 labeled neutrophils by F4/80 labeled macrophages. (E) Summarizes the \% macrophages that internalized neutrophils \pm SEM where each dot represents the macrophages from one mouse, conducted over 2 experimental replicates. A two-tailed student T-test was performed to determine statistical significance at each timepoint. $*=p>0.05, * *=p>0.01, * * *=p>0.001$. 
A.

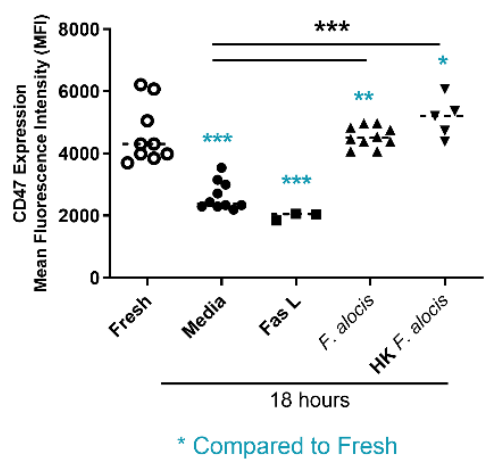

C.

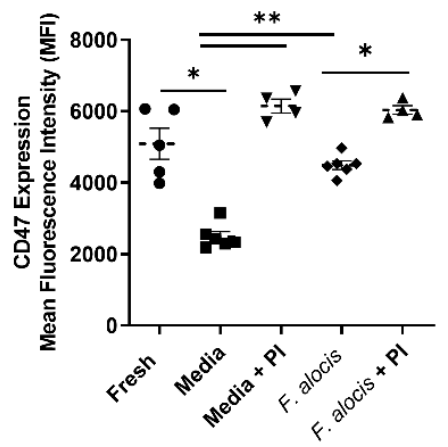

B.

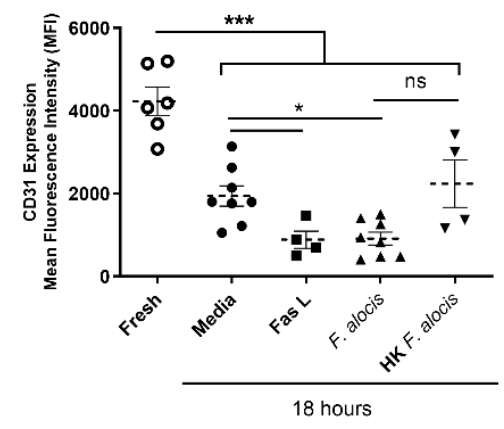

D.

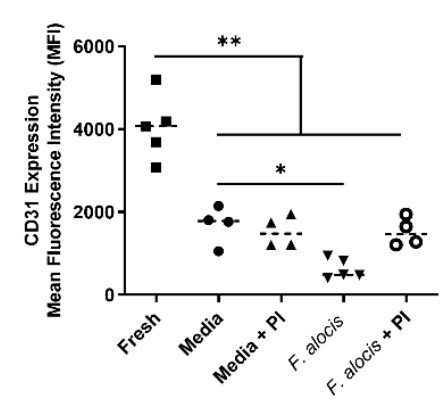

Figure 5.2: $F$. alocis-challenged human neutrophils expression of "don't eat me" signals. Fresh neutrophils or cells aged for 18 hours in media or with FasL, F. alocis or heat killed (HK) F. alocis (MOI 10) were stained for (A) CD47 or (B) CD31 expression. Data show the mean channel fluorescence of expression \pm SEM from 3-10 independent experiments. Then, In addition to the conditions from A and B, some neutrophils were also pre-treated with protease inhibitors (PI) followed by culture for 18 hours in media or with F. alocis and staining for (C) CD47 or (D) CD31 expression. One-way ANOVAs with Tukey post-hoc testing was performed on all plots to determine statistical significance. ${ }^{*}=$ $\mathrm{p}>0.05, * *=\mathrm{p}>0.01, * * *=\mathrm{p}>0.001$. 


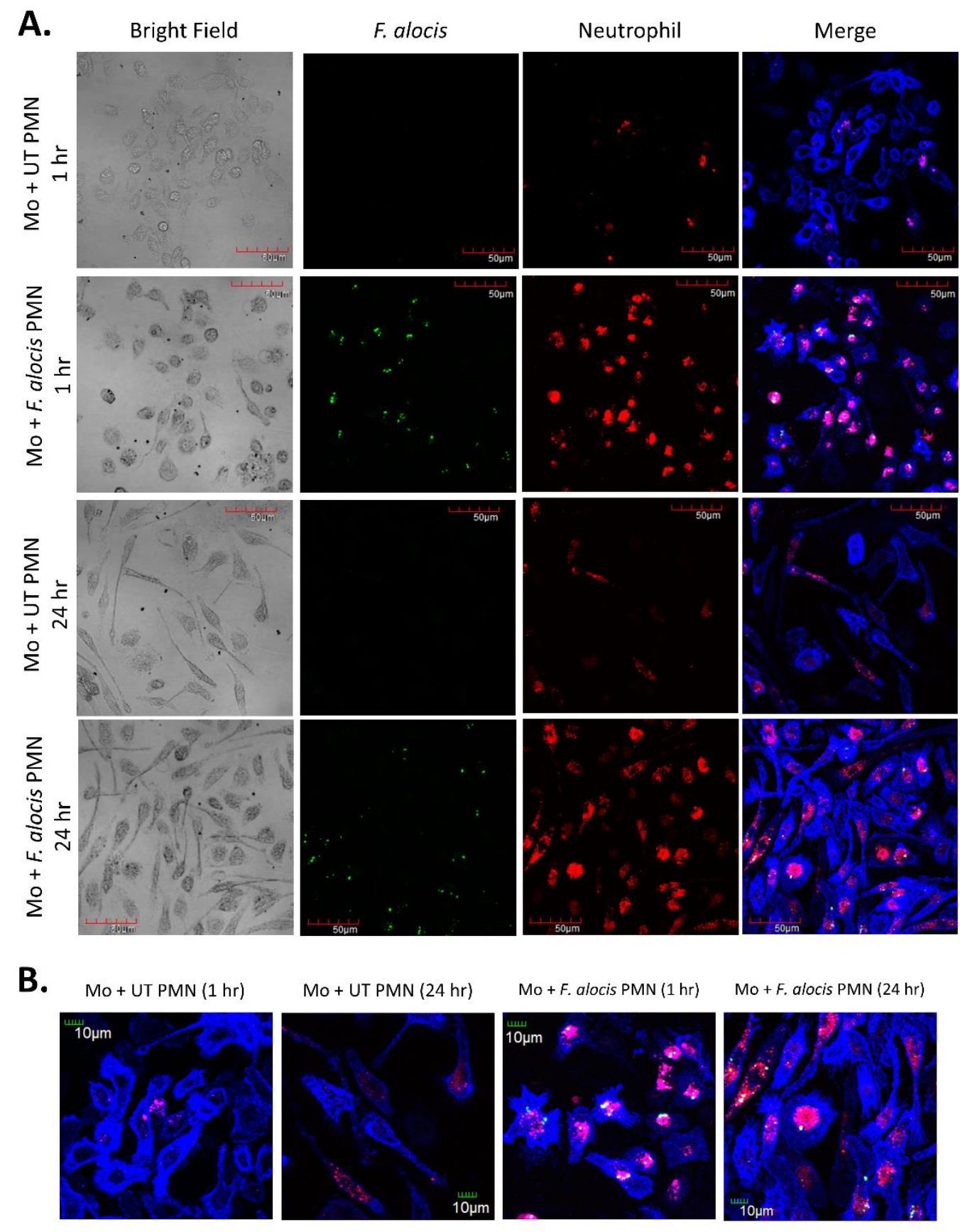

Figure 5.3: $F$. alocis-challenged human neutrophils persist in macrophages after 24 hours. 
Figure 5.3: PKH-26 labeled neutrophils (Red) were aged for 18 hours in media or with CFSE F. alocis (Green) and then fed to macrophages for 1 hour. After washing off noningested neutrophils, the cells were left to culture for an additional 23 hours or fixed and macrophages stained for F4/80 (Blue). (A) Shows representative Z-stack images of macrophages from these conditions. (B) Shows zoomed sections from the merged images in A. 
A.

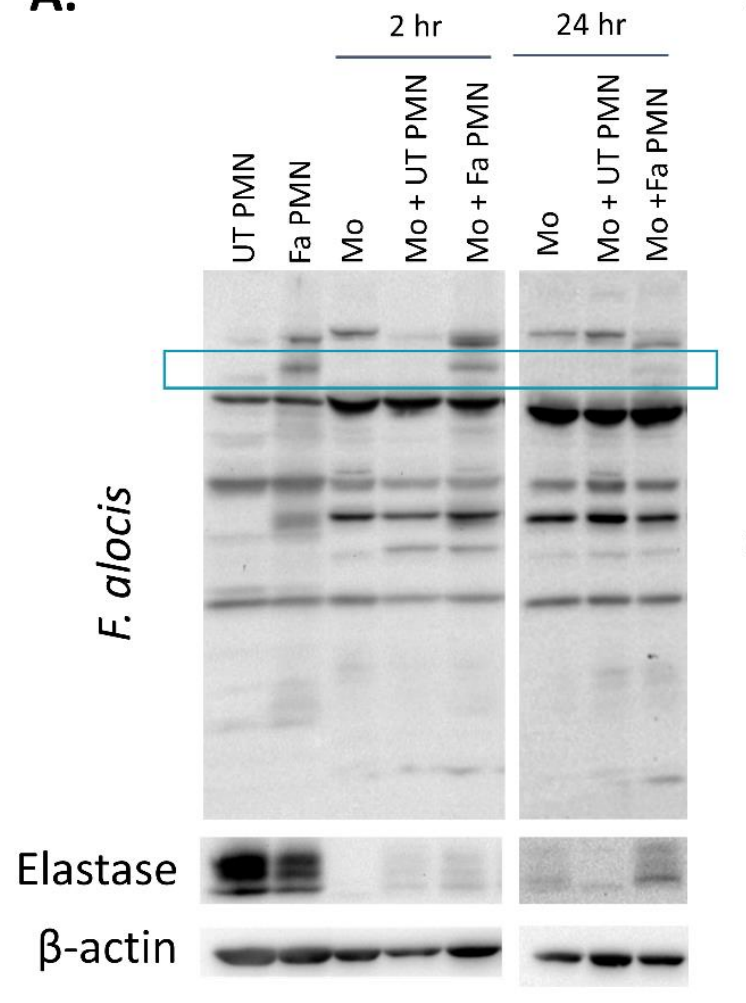

B.

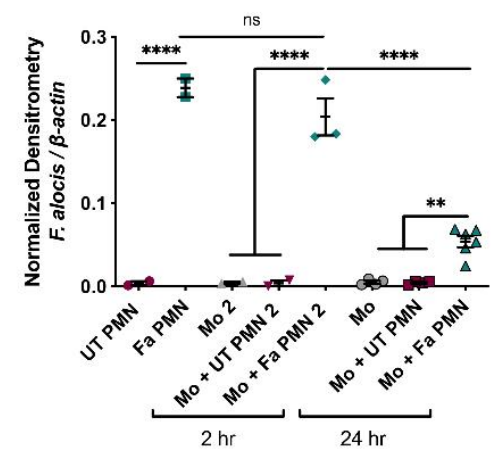

C.

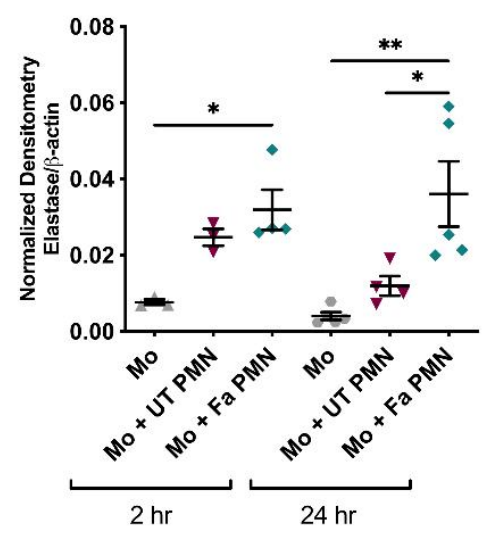

Figure 5.4: $F$. alocis challenged human neutrophils are not efficiently degraded by macrophages. 
Figure 5.4: Neutrophils (PMN) were aged for 18 hours in media (UT) or with $F$. alocis (Fa), and then fed to macrophages (Mo) for 1 hour. After washing off non-ingested neutrophils, the cells were left to culture for 1 or 23 more hours. (A) Whole cell lysates were probed for $F$. alocis, Elastase, or $\beta$ actin. Lysates are from the non-ingested neutrophils aged for 18 hours in media or with $F$. alocis, unchallenged macrophages (Mo), macrophages fed media-cultured neutrophils (Mo + UT PMN), or macrophages fed $F$. alocis-cultured neutrophils (Fa + UT PMN). Box highlights unique F. alocis protein used for blot quantification as region of interest (ROI). Data are plotted as the mean \pm SEM of the normalized densitometry of $(\mathbf{B})$ the boxed $F$. alocis protein or $(\mathbf{C})$ elastase. Each dot represents a mouse and data was collected from 2 experimental replicates. One-way ANOVAs with Tukey post-hoc testing were performed on B \& C to determine statistical significance. 
A.

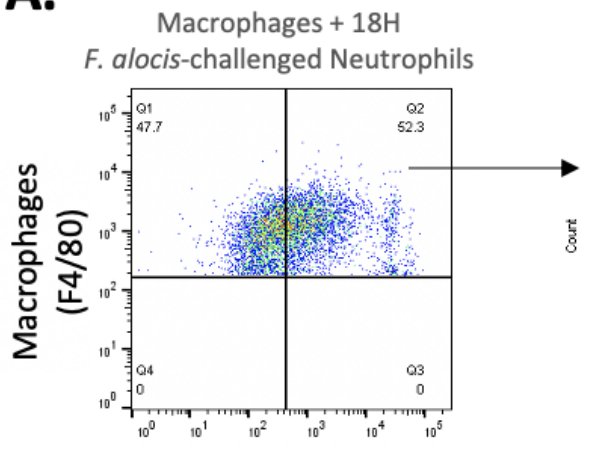

Neutrophils (PKH-26)

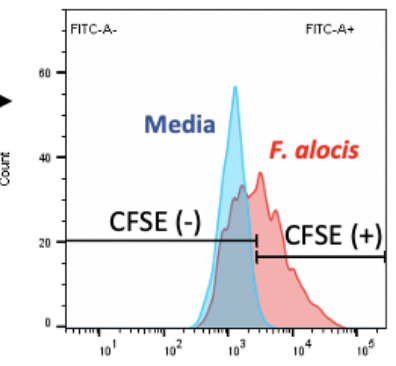

Intensity of CFSE
B.

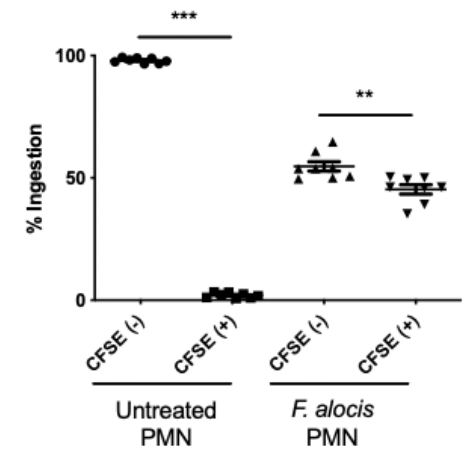

C.

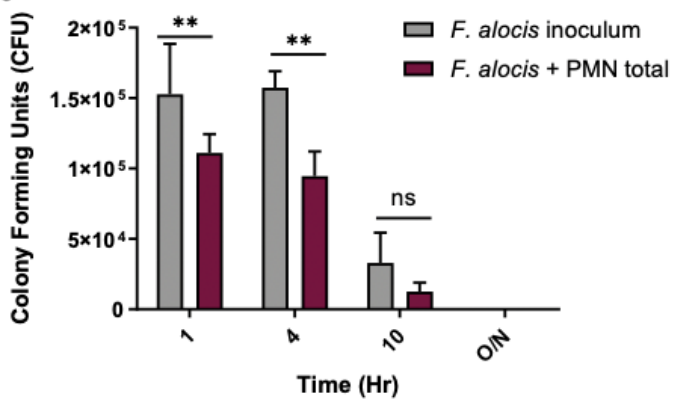

D.

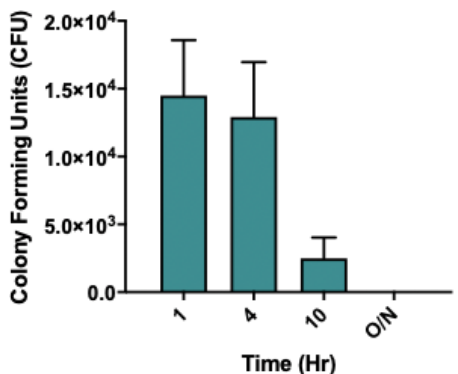

E.

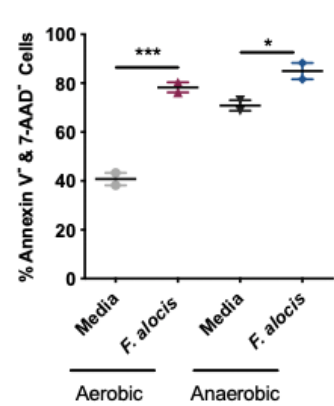

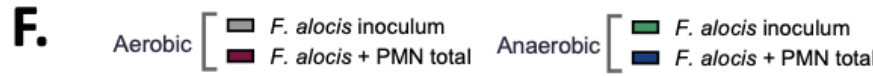

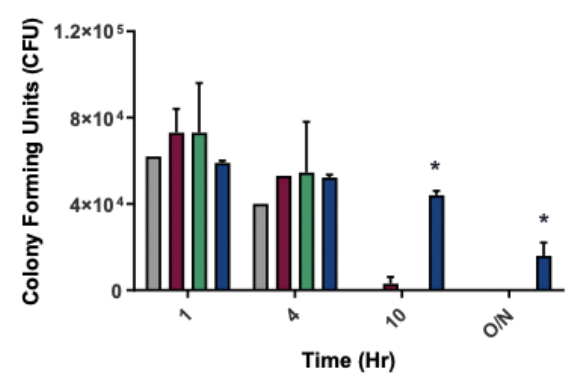

G. Aerobic Intracellular $\mathrm{Fa} \square$ Anaerobic Intracellular $\mathrm{Fa}$

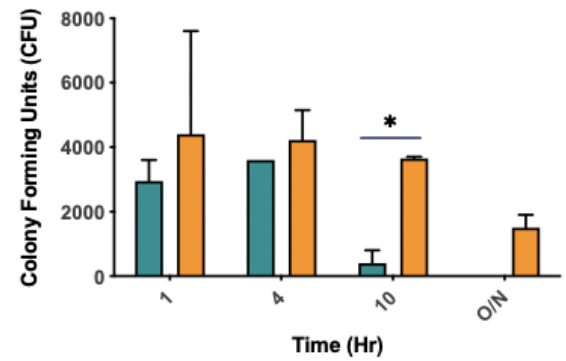

Figure 5.5: F. alocis neutrophils as "trojan horses". 
Figure 5.5: (A) Dot plots show gating strategy to determine the percent population of macrophages that engulfed neutrophils (F4/80+, PKH-26+, Q2). Q2 macrophages from both the media-treated neutrophils condition and CFSE-labeled $F$. alocis-treated neutrophil condition, were plotted by their CFSE intensity in a histogram. Two gates differentiate macrophages that ingested neutrophils that did not contain bacteria (CFSE(-)) and macrophages that ingested neutrophils that contained $F$. alocis $(\operatorname{CFSE}(+))$. (B) Shows the $\%$ of Q2 macrophages \pm SEM that ingested $F$. alocis negative CFSE(-) or $F$. alocis positive $\mathrm{CFSE}(+)$ neutrophils from both conditions. Each dot $(\mathrm{N}=6)$ represents a mouse from 2 experimental replicates. (C) Data show the total colony forming units (CFU) from the aerobic culture of $F$. alocis inoculum and $F$. alocis cultured with neutrophils for 1, 4, 10 hours and overnight. (D) Shows CFUs recovered from F. alocis cultured with neutrophils where extracellular bacteria was washed off. C and D show average CFUs \pm SEM from 3 independent experiments. (E) Neutrophil viability was assessed after 24 hours of aerobic or anaerobic culture in media or with $F$. alocis by Annexin V/7AAD staining. Data are plotted as the mean percent viable (Q4: Annexin V-, 7AAD-) neutrophils \pm SEM from two independent experiments. (F) Shows total colony forming units (CFU) from the culture of $F$. alocis inoculum and $F$. alocis cultured with neutrophils under aerobic and anaerobic conditions for the times shown. (G) Shows intracellular CFUs recovered after aerobic and anaerobic culture. Both $\mathrm{F}$ and $\mathrm{G}$ represent the average CFUs \pm SEM from 2 independent experiments. One-way ANOVAs with Tukey post-hoc testing was performed on B, D, and E to determine statistical significance. A two-way ANOVA with Bonferroni post-hoc testing was performed on $\mathrm{C}, \mathrm{F}$ and $\mathrm{G}$ to determine statistical significance. $*=\mathrm{p}>0.05$, $* *=\mathrm{p}>0.01, * * *=\mathrm{p}>0.001$ 

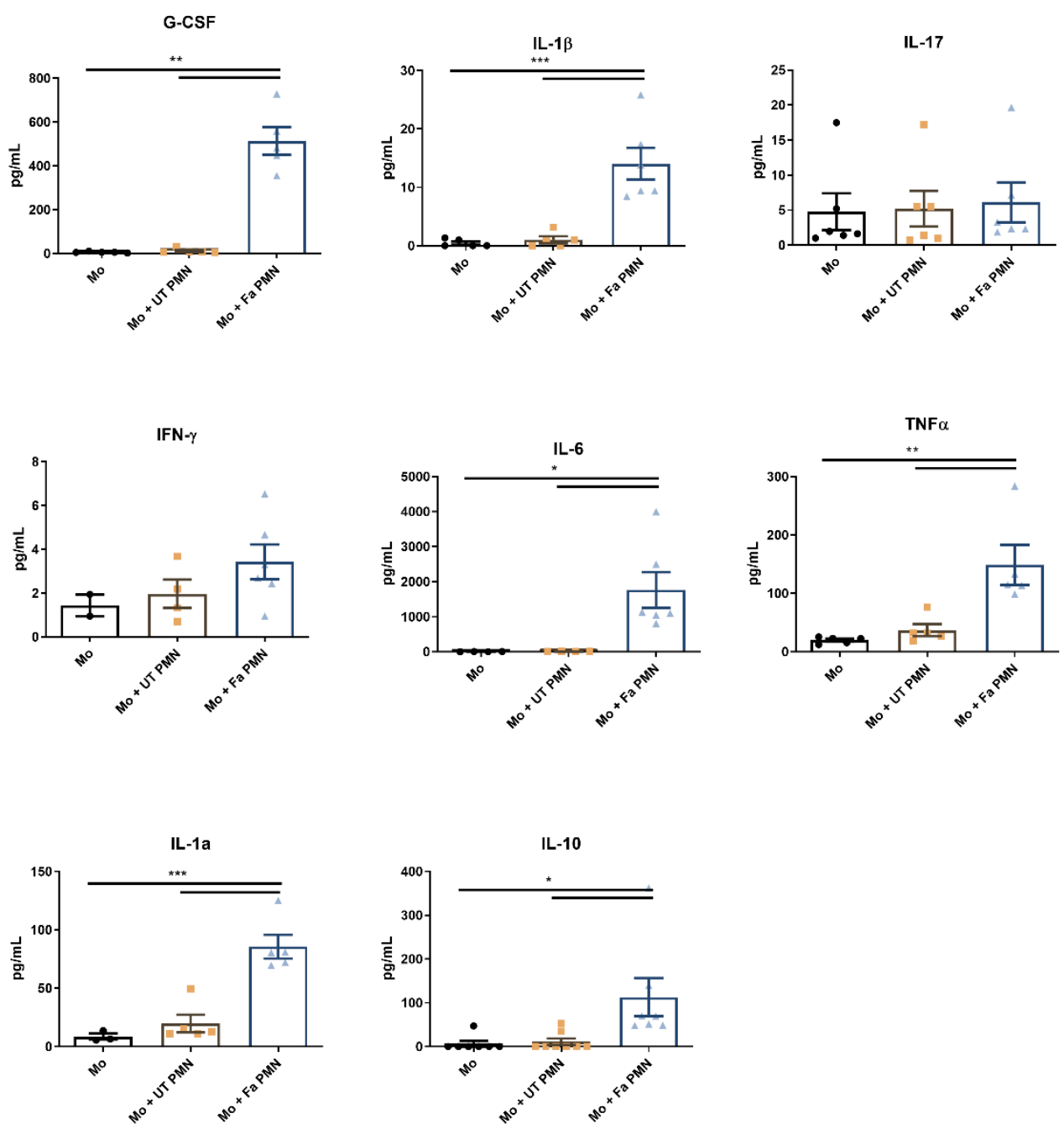

Figure 5.6: Cytokine profile of efferocytic macrophages after ingestion of $F$. alocis neutrophils. Neutrophils (PMN) were aged for 18 hours in media (UT) or with $F$. alocis $(\mathrm{Fa})$, and then fed to macrophages (Mo) for 1 hour. After washing off non-ingested neutrophils, the cells were left to culture for 23 more hours. The supernatants were collected from 7 mice over 2 independent experiments and tested using a 32-analyte multiplex array. The targets that could be detected are shown here. One-way ANOVAs with Tukey post-hoc testing were performed to determine statistical significance. $*=p>$ $0.05, * *=\mathrm{p}>0.01, * * *=\mathrm{p}>0.001$. 

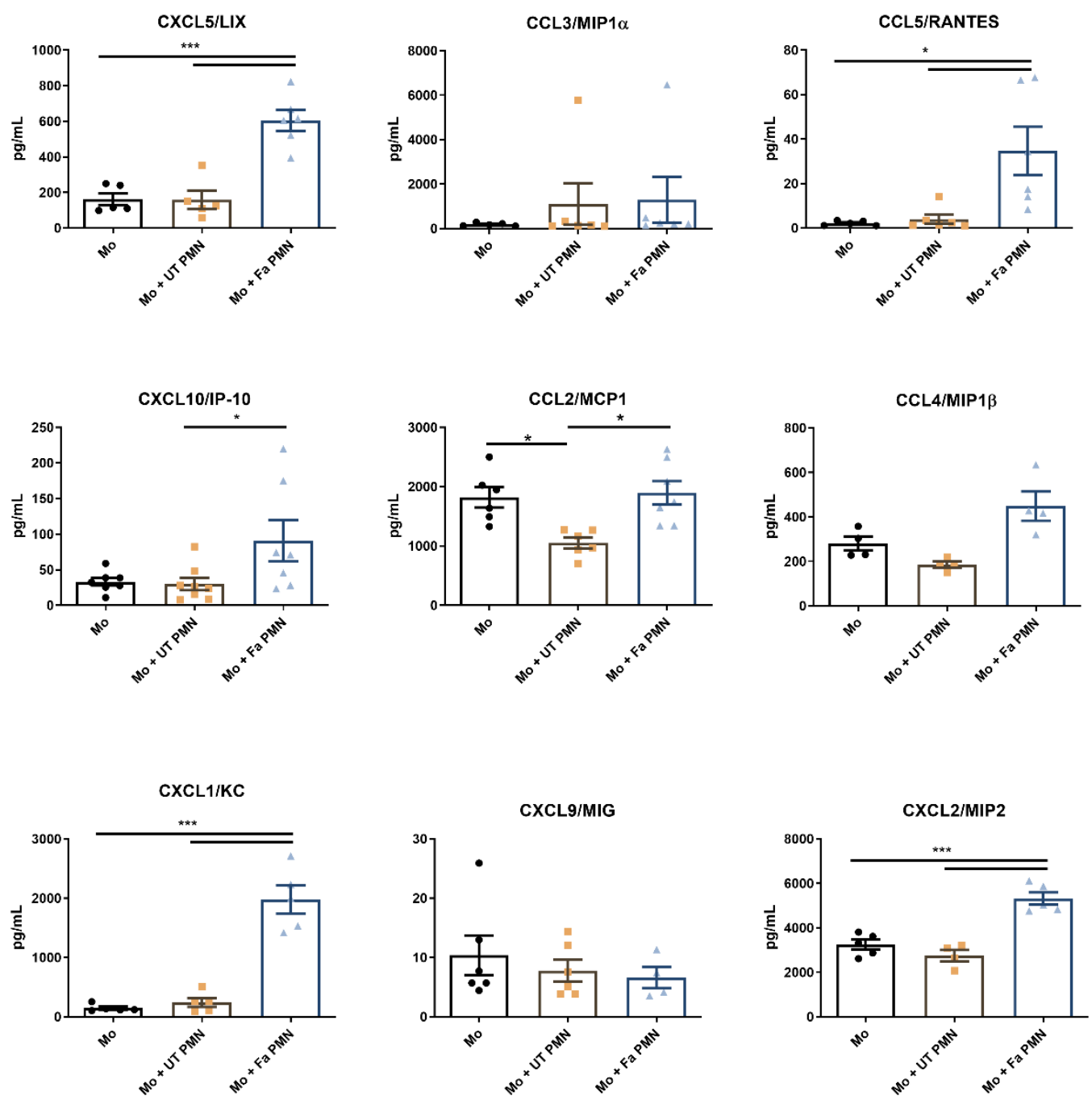

Figure 5.7: Chemokine profile of efferocytic macrophages after ingestion of $F$. alocis

neutrophils. Neutrophils (PMN) were aged for 18 hours in media (UT) or with F. alocis (Fa), and then fed to macrophages (Mo) for 1 hour. After washing off non-ingested neutrophils, the cells were left to culture for 23 more hours. The supernatants were collected from 7 mice over 2 independent experiments and tested using a 32-analyte multiplex array. The targets that could be detected are shown here. One-way ANOVAs with Tukey post-hoc testing were performed to determine statistical significance. $*=p>$ $0.05, * *=\mathrm{p}>0.01, * * *=\mathrm{p}>0.001$. 


\section{CHAPTER 6:}

\section{DISCUSSION AND FUTURE DIRECTIONS}

Periodontitis is an irreversible, bacteria-induced, chronic inflammatory disease that compromises the integrity of tooth-supporting tissues and adversely impacts systemic health. Neutrophils are the immune system's first line of defense against bacteria in the oral cavity, where their microbicidal functions typically protect the host against periodontal disease. However, periodontal pathogens have evolved mechanisms to resist neutrophil microbicidal mechanisms while still propagating inflammation, which provides essential nutrients for the bacteria to proliferate and cause disease. Advances in sequencing technologies have recognized several newly appreciated bacteria associated with periodontal lesions, like the Gram-positive anaerobic rod, Filifactor alocis.

With the discovery of these emerging oral bacterial species, there is also a growing need to (1) assess their pathogenic potential and (2) determine their contribution to disease progression in order to develop adequate therapies against periodontal disease and its associated comorbidities. Previous research from our laboratory initially characterized some of the pathogenic behaviors of F. alocis toward neutrophils. The work from this dissertation is an extension of this goal and was also aimed at understanding how the neutrophil functions were modulated. To get a global picture of changes in neutrophils during $F$. alocis challenge, I conducted a time course transcriptome study. Analysis of this dataset demonstrated that $F$. alocis has a pervasive effect on neutrophil gene expression. 
By combining results from the transcriptome and F. alocis-modulated neutrophil effector functions, here I will discuss our working model of how $F$. alocis may be contributing to the chronic inflammation and the progression of periodontal disease (Figure 6.1).

First of all, F. alocis is recognized by human neutrophils through ligation of TLR2/6. The resultant signaling modulates neutrophil migration patterns [275] and induces granule exocytosis. Both of these functions have implications for tissue destruction in the periodontium. Compounding the inflammatory response is the fact that previous work showed that $F$. alocis could survive within neutrophils up to 4 hours [284]. Using colony forming units, I confirmed these findings and found that under anaerobic conditions, viable F. alocis can be recovered after 24 hours of culture with human neutrophils. Additionally, the bacteria's survival is extended when neutrophils are present, suggesting that neutrophils contribute to favorable conditions for the bacterium and/or act as a niche for persistence.

The intracellular persistence of $F$. alocis in human neutrophils has been attributed to minimal ROS production and a delay in phagosome maturation [284]. Both of these phenotypes were confirmed in murine neutrophils, which is an important detail for when studies progress into the animal models of periodontitis. Regardless of defective TLR2 or MyD88 signaling, ROS production was not restored nor further dampened. However, granule fusion with the phagosome showed some interesting differences. Compared to wild-type neutrophils, the maturation of the phagosome was accelerated in F. alocis phagosomes from TLR2 deficient mice, whereas neutrophils from MyD88 ${ }^{-/-}$mice were not able to digest viable $F$. alocis after 24 hours. For optimal antimicrobial capacity, it is critical that both the NADPH oxidase is activated and neutrophil granules fuse with the phagosome [321]. Thus, more studies will be needed to tease apart the signaling cascades that lead to 
minimal ROS generation and a delay in granule fusion with the phagosome. Of note, the data from the transcriptome shows that $F$. alocis challenge significantly downregulates the expression of genes in the vesicle-mediated transport, regulated exocytosis and neutrophil degranulation. For example, multiple members of the Rab and Syntaxin families were differentially expressed, but it will be necessary to determine if their functions are impaired. Together with the measured decrease in granule mobilization due to $F$. alocis infection, this is a promising area that merits more research.

One of the top hits from the transcriptome was inflammation, which is expected in the context of a chronic inflammatory bacterial infection. Amongst the significant

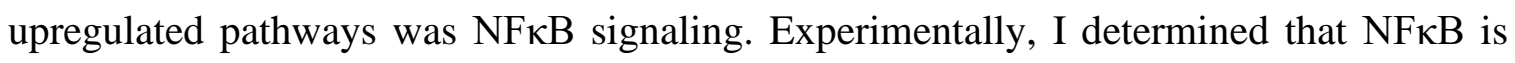
activated very quickly after $F$. alocis challenge, and that TLR2 and MyD88 signaling is critical for the production of cytokines and chemokines. These pro-inflammatory mediators are especially important in the context of periodontitis because the chemokines will recruit more inflammatory cells to the oral cavity, and the pro-inflammatory cytokines will prime incoming cells, further enhancing their capacity to drive tissue destruction. Additionally, I found that the interaction between neutrophils and F. alocis produces pro-survival soluble factors that extend the neutrophil lifespan, which also extends the time they can drive the dysregulated inflammatory environment.

Despite driving a pro-inflammatory program, $F$. alocis also seems to be striking a balance between pro- and anti-inflammatory responses. For example, in comparison to other oral pathogens, $F$. alocis does not induce NETs production and inhibits the release of NETs by the potent neutrophil activator, PMA [276]. Neutrophils stimulated with the oral pathogen also produced a significant amount of the anti-inflammatory cytokine, IL- 
10. Similarly, in the transcriptome data, the upregulated inflammatory pathways from early timepoints became significantly downregulated during the later time points, suggesting that F. alocis may be gaining control of the cell and dampening inflammatory processes at later timepoints. Data from this dissertation exemplified this concept further by showing that $F$. alocis dampened the activation of TNF $\alpha$-mediated MAPK signaling and as a result, limited the production of IL-8. At later timepoints, $F$. alocis challenge resulted in the significant downregulation of signal transduction pathways like MAPK, GPCR, Rho GTPases, PI3K, PTEN, AKT, and PKA, which could have significant impacts on neutrophil functions towards secondary stimuli.

I found that neutrophils stimulated with $F$. alocis for up to 18 hours retain their phagocytic capacity and their ability to generate ROS in response to secondary stimuli, like S. aureus. However, there are other neutrophil functions I have not assessed downstream of $F$. alocis stimulation. Furthermore, it would be interesting to assess whether neutrophil negative feedback loops are activated with different kinetics or to different magnitudes during $F$. alocis stimulation to enact the changes in responsiveness of these neutrophils. The microbiota associated with disease is influenced by the virulent traits of specialized microorganisms that raise the pathogenicity and survival of the entire community. While it has been shown that $F$. alocis can protect other species from complement killing [64], one of the aspects of $F$. alocis infection I have not addressed in depth is the bystander effects of $F$. alocis-influenced neutrophil functions. Are F. alocis-controlled neutrophils defective at eliminating other oral bacteria? At early timepoints, the dampened ROS response is limited to F. alocis phagosomes [284], but is the thwarted granule movement wide-spread in the cell and could it protect other internalized bacteria? 
Neutrophils that encounter $F$. alocis have prolonged lifespans and retain their functional capabilities for a longer time than unstimulated cells. The next step in the apoptosis story is to determine how/where the apoptotic pathways are specifically targeted by $F$. alocis, since caspase 3,8 , and 9 were minimally activated in $F$. alocis treated neutrophils. Furthermore, can $F$. alocis pre-treatment of the neutrophils prevent activation of apoptotic pathways by known cell death agonists? The effect on neutrophil survival was even more pronounced in anaerobic conditions. Since neutrophils encounter hypoxic conditions as periodontitis develops and the periodontal pocket deepens, more work to elucidate their responses in this environment will give a better picture of neutrophils behavior in the progression of the disease. Despite the extended lifespan and functional capacity of neutrophils when challenged with $F$. alocis, to our surprise, the infected neutrophils were more avidly engulfed by macrophages compared to media-cultured neutrophils. I now believe this effect is due to the loss of CD31 expression on the surface of F. alocis-challenged neutrophils, which is driven by an F. alocis-derived protease. More experiments are needed to prove our hypothesis, as well as characterize this protease.

The progression of neutrophil cell death into apoptosis and subsequent efferocytosis is supposed to be a non-inflammatory process. However, macrophages that engulfed $F$. alocis-challenged neutrophils produced a robust pro-inflammatory cytokine and chemokine response. Additionally, F. alocis-neutrophils were degraded to a lesser extent than media-cultured neutrophils. The differences in cargo degradation within efferosomes between untreated or $F$. alocis treated neutrophils is an area that warrants more characterization. Several markers are available to track the maturation of efferosomes like LC3, LAMP1, and lysotracker, so the presence of these should be assessed in our system. 
Decreased efferosome maturation has two important implications that should also be tested. The first is that under the correct oxygen conditions, F. alocis may be using neutrophils as "trojan horses" to enter a longer-lived host. Corroborating this hypothesis is unpublished data from our laboratory shows that $F$. alocis can also establish a niche within human macrophages. The second implication is that if dysregulated clearance of apoptotic cells can accelerate the progression of autoimmune disorders [427]. There is a strong correlation between limited proteolysis of phagosomal cargo and cross-presentation of antigenic peptides by major histocompatibility complex proteins that activate adaptive immune responses [447, 461]. Pathways for autoinflammatory diseases like rheumatoid arthritis were significant hits in the transcriptome of $F$. alocis-challenged neutrophils.

In conclusion, the data from this dissertation asserts that $F$. alocis manipulates neutrophil gene expression profiles, signaling cascades and antimicrobial mechanisms to survive intracellularly, extend neutrophil lifespan, and cause the release of proinflammatory cytokines. The nefarious effects of $F$. alocis on neutrophils also trickle down to efferocytic macrophages and potentiate more inflammation in the oral cavity. Finally, our results are the first to delineate the $F$. alocis strategies to control neutrophil effector functions by interrupting intracellular signaling and will enhance our understanding of how oral bacteria undermine the innate immune system. 


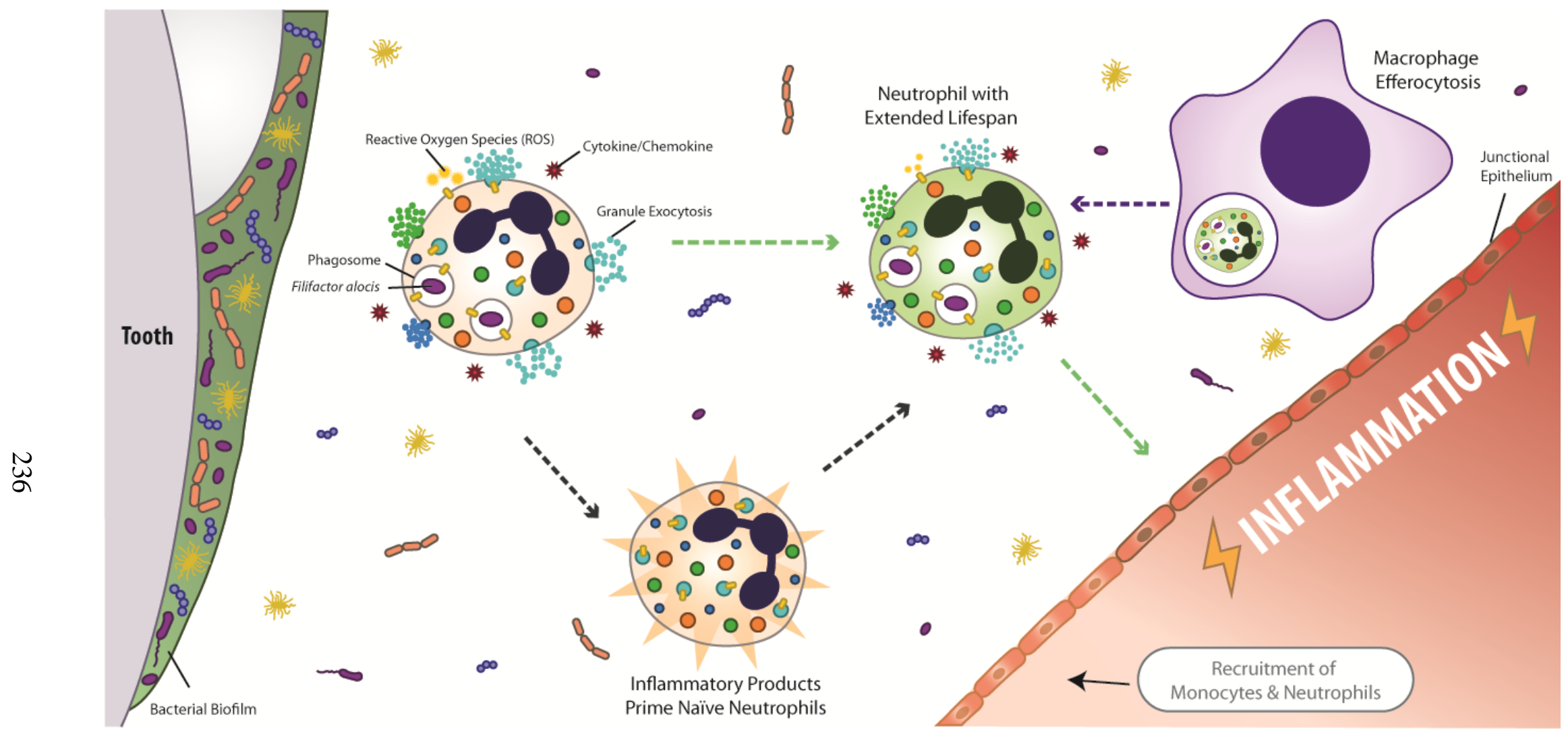

Figure 6.1 Working model of $F$. alocis effects on neutrophils. 


\section{REFERENCES}

1. $\quad$ Eke, P.I., et al., Periodontitis in US Adults: National Health and Nutrition Examination Survey 2009-2014. The Journal of the American Dental Association, 2018. 149(7): p. 576588.e6.

2. Pihlstrom, B.L., B.S. Michalowicz, and N.W. Johnson, Periodontal diseases. Lancet, 2005. 366(9499): p. 1809-20.

3. Douglass, C.W., Risk assessment and management of periodontal disease. The Journal of the American Dental Association, 2006. 137: p. S27-S32.

4. Lamont, R.J. and G. Hajishengallis, Polymicrobial synergy and dysbiosis in inflammatory disease. Trends Mol Med, 2015. 21(3): p. 172-83.

5. Short, F.L., S.L. Murdoch, and R.P. Ryan, Polybacterial human disease: the ills of social networking. Trends Microbiol, 2014. 22(9): p. 508-16.

6. Michie, K.L., D.M. Cornforth, and M. Whiteley, Bacterial tweets and podcasts \#signaling\#eavesdropping\#microbialfightclub. Mol Biochem Parasitol, 2016. 208(1): p. 41-8.

7. Murray, J.L., et al., Mechanisms of synergy in polymicrobial infections. J Microbiol, 2014. 52(3): p. 188-99.

8. Hajishengallis, G. and R.J. Lamont, Dancing with the Stars: How Choreographed Bacterial Interactions Dictate Nososymbiocity and Give Rise to Keystone Pathogens, Accessory Pathogens, and Pathobionts. Trends Microbiol, 2016. 24(6): p. 477-489.

9. Hajishengallis, G., Immunomicrobial pathogenesis of periodontitis: keystones, pathobionts, and host response. Trends in Immunology, 2013(Suppl. 11).

10. Hajishengallis, G. and R.J. Lamont, Beyond the red complex and into more complexity: the polymicrobial synergy and dysbiosis (PSD) model of periodontal disease etiology. Molecular Oral Microbiology, 2012. 27(6): p. 409-419.

11. Hajishengallis, G., et al., Low-abundance biofilm species orchestrates inflammatory periodontal disease through the commensal microbiota and complement. Cell Host Microbe, 2011. 10(5): p. 497-506.

12. Kebschull, M., R.T. Demmer, and P.N. Papapanou, "Gum Bug, Leave My Heart Alone!"Epidemiologic and Mechanistic Evidence Linking Periodontal Infections and Atherosclerosis. Journal of Dental Research, 2010. 89(9): p. 879-902.

13. Bingham, C.O.I. and M. Moni, Periodontal disease and rheumatoid arthritis: the evidence accumulates for complex pathobiologic interactions. Current Opinion in Rheumatology, 2013. 25(3): p. 345-353.

14. Hajishengallis, G., Periodontitis: from microbial immune subversion to systemic inflammation. Nat Rev Immunol, 2015. 15(1): p. 30-44.

15. Sureda, A., et al., Oral microbiota and Alzheimer's disease: Do all roads lead to Rome? Pharmacol Res, 2020. 151: p. 104582.

16. Offenbacher, S., et al., Results from the Periodontitis and Vascular Events (PAVE) Study: a pilot multicentered, randomized, controlled trial to study effects of periodontal therapy in a secondary prevention model of cardiovascular disease. J Periodontol, 2009. 80(2): p. 190-201.

17. Genco, R.J. and T.E. Van Dyke, Prevention: Reducing the risk of CVD in patients with periodontitis. Nat Rev Cardiol, 2010. 7(9): p. 479-80. 
18. Liu, H., R.W. Redline, and Y.W. Han, Fusobacterium nucleatum induces fetal death in mice via stimulation of TLR4-mediated placental inflammatory response. J Immunol, 2007. 179(4): p. 2501-8.

19. Kozarov, E.V., et al., Human atherosclerotic plaque contains viable invasive Actinobacillus actinomycetemcomitans and Porphyromonas gingivalis. Arterioscler Thromb Vasc Biol, 2005. 25(3): p. e17-8.

20. Arimatsu, K., et al., Oral pathobiont induces systemic inflammation and metabolic changes associated with alteration of gut microbiota. Sci Rep, 2014. 4: p. 4828.

21. Hajishengallis, G., Immunomicrobial pathogenesis of periodontitis: keystones, pathobionts, and host response. Trends Immunol, 2014. 35(1): p. 3-11.

22. Berezow, A.B. and R.P. Darveau, Microbial shift and periodontitis. Periodontol 2000, 2011. 55(1): p. 36-47.

23. Socransky, S.S. and A.D. Haffajee, Evidence of bacterial etiology: a historical perspective. Periodontol 2000, 1994. 5: p. 7-25.

24. Feres, M., et al., Microbiological basis for periodontal therapy. J Appl Oral Sci, 2004. 12(4): p. 256-66.

25. Listgarten, M.A., Structure of the microbial flora associated with periodontal health and disease in man. A light and electron microscopic study. J Periodontol, 1976. 47(1): p. 118.

26. Listgarten, M.A. and L. Hellden, Relative distribution of bacteria at clinically healthy and periodontally diseased sites in humans. J Clin Periodontol, 1978. 5(2): p. 115-32.

27. Tsai, C.Y., et al., A rapid DNA probe test compared to culture methods for identification of subgingival plaque bacteria. J Clin Periodontol, 2003. 30(1): p. 57-62.

28. Deng, W., et al., The use of molecular techniques based on ribosomal RNA and DNA for rumen microbial ecosystem studies: a review. Mol Biol Rep, 2008. 35(2): p. 265-74.

29. Anderson, I.C. and J.W. Cairney, Diversity and ecology of soil fungal communities: increased understanding through the application of molecular techniques. Environ Microbiol, 2004. 6(8): p. 769-79.

30. Dahlen, G. and A. Leonhardt, A new checkerboard panel for testing bacterial markers in periodontal disease. Oral Microbiol Immunol, 2006. 21(1): p. 6-11.

31. Socransky, S.S., et al., Microbial complexes in subgingival plaque. J Clin Periodontol, 1998. 25(2): p. 134-44.

32. Paster, B.J., et al., Bacterial diversity in human subgingival plaque. J Bacteriol, 2001. 183(12): p. 3770-83.

33. Paster, B.J., et al., The breadth of bacterial diversity in the human periodontal pocket and other oral sites. Periodontol 2000, 2006. 42: p. 80-7.

34. Kumar, P.S., et al., Changes in periodontal health status are associated with bacterial community shifts as assessed by quantitative 16S cloning and sequencing. J Clin Microbiol, 2006. 44(10): p. 3665-73.

35. Dewhirst, F.E., et al., The human oral microbiome. J Bacteriol, 2010. 192(19): p. 5002-17.

36. Abusleme, L., et al., The subgingival microbiome in health and periodontitis and its relationship with community biomass and inflammation. ISME J, 2013. 7(5): p. 1016-25.

37. Aas, J.A., et al., Defining the normal bacterial flora of the oral cavity. J Clin Microbiol, 2005. 43(11): p. 5721-32.

38. Griffen, A.L., et al., Distinct and complex bacterial profiles in human periodontitis and health revealed by $16 S$ pyrosequencing. ISME J, 2012. 6(6): p. 1176-85.

39. Krishnan, K., T. Chen, and B.J. Paster, A practical guide to the oral microbiome and its relation to health and disease. Oral Dis, 2017. 23(3): p. 276-286.

40. Socransky, S.S. and A.D. Haffajee, Periodontal microbial ecology. Periodontol 2000, 2005. 38: p. 135-87. 
41. Han, Y.W., Fusobacterium nucleatum: a commensal-turned pathogen. Curr Opin Microbiol, 2015. 23: p. 141-7.

42. Perez-Chaparro, P.J., et al., Newly identified pathogens associated with periodontitis: a systematic review. J Dent Res, 2014. 93(9): p. 846-58.

43. Cato, E.P., Moore, L. V. H., and Moore, W. E. C. , Fusobacterium alocis sp. nov. and Fusobacterium sulci sp. nov. from the human gingival sulcus. International Journal of Systemic Bacteriology, 1985. 35(4): p. 475-477.

44. Jalava, J. and E. Eerola, Phylogenetic analysis of Fusobacterium alocis and Fusobacterium sulci based on 16S rRNA gene sequences: proposal of Filifactor alocis (Cato, Moore and Moore) comb. nov. and Eubacterium sulci (Cato, Moore and Moore) comb. nov. Int J Syst Bacteriol, 1999. 49 Pt 4: p. 1375-9.

45. Kumar, P.S., et al., New bacterial species associated with chronic periodontitis. J Dent Res, 2003. 82(5): p. 338-44.

46. Deng, Z.L., et al., Dysbiosis in chronic periodontitis: Key microbial players and interactions with the human host. Sci Rep, 2017. 7(1): p. 3703.

47. Ikeda, E., et al., Japanese subgingival microbiota in health vs disease and their roles in predicted functions associated with periodontitis. Odontology, 2019.

48. Schlafer, S., et al., Filifactor alocis--involvement in periodontal biofilms. BMC Microbiol, 2010. 10: p. 66.

49. Siqueira, J.F., Jr. and I.N. Rocas, Simultaneous detection of Dialister pneumosintes and Filifactor alocis in endodontic infections by $16 S$ rDNA-directed multiplex PCR. J Endod, 2004. 30(12): p. 851-4.

50. Gomes, B.P., et al., Molecular analysis of Filifactor alocis, Tannerella forsythia, and treponema denticola associated with primary endodontic infections and failed endodontic treatment. J Endod, 2006. 32(10): p. 937-40.

51. Siqueira, J.F., Jr., et al., Bacteria in the apical root canal of teeth with primary apical periodontitis. Oral Surg Oral Med Oral Pathol Oral Radiol Endod, 2009. 107(5): p. 721-6.

52. Zhang, C., et al., Microbial diversity in failed endodontic root-filled teeth. Chin Med J (Engl), 2012. 125(6): p. 1163-8.

53. da Silva, E.S., et al., Microbiological diversity of peri-implantitis biofilm by Sanger sequencing. Clin Oral Implants Res, 2014. 25(10): p. 1192-9.

54. Schulz, S., et al., Comparison of the oral microbiome of patients with generalized aggressive periodontitis and periodontitis-free subjects. Arch Oral Biol, 2019. 99: p. 169176.

55. Liu, G., et al., Measuring the subgingival microbiota in periodontitis patients: Comparison of the surface layer and the underlying layers. Microbiol Immunol, 2020. 64(2): p. 99-112.

56. Naginyte, M., et al., Enrichment of periodontal pathogens from the biofilms of healthy adults. Sci Rep, 2019. 9(1): p. 5491.

57. Chen, H., et al., A Filifactor alocis-centered co-occurrence group associates with periodontitis across different oral habitats. Sci Rep, 2015. 5: p. 9053.

58. Aruni, A.W., F. Roy, and H.M. Fletcher, Filifactor alocis has virulence attributes that can enhance its persistence under oxidative stress conditions and mediate invasion of epithelial cells by porphyromonas gingivalis. Infect Immun, 2011. 79(10): p. 3872-86.

59. Wang, Q., et al., Oral community interactions of Filifactor alocis in vitro. PLoS One, 2013. 8(10): p. e76271.

60. Uematsu, H., et al., Degradation of arginine and other amino acids by butyrate-producing asaccharolytic anaerobic Gram-positive rods in periodontal pockets. Arch Oral Biol, 2003. 48(6): p. 423-9.

61. Aruni, A.W., et al., Filifactor alocis--a new emerging periodontal pathogen. Microbes Infect, 2015. 17(7): p. 517-30. 
62. Aruni, A.W., et al., Proteome analysis of coinfection of epithelial cells with Filifactor alocis and Porphyromonas gingivalis shows modulation of pathogen and host regulatory pathways. Infect Immun, 2014. 82(8): p. 3261-74.

63. Aruni, A.W., et al., Proteome variation among Filifactor alocis strains. Proteomics, 2012. 12(22): p. 3343-64.

64. Jusko, M., et al., FACIN, a Double-Edged Sword of the Emerging Periodontal Pathogen Filifactor alocis: A Metabolic Enzyme Moonlighting as a Complement Inhibitor. J Immunol, 2016. 197(8): p. 3245-3259.

65. Kim, H.Y., et al., Characterization and immunostimulatory activity of extracellular vesicles from Filifactor alocis. Mol Oral Microbiol, 2020. 35(1): p. 1-9.

66. Moffatt, C.E., et al., Filifactor alocis interactions with gingival epithelial cells. Mol Oral Microbiol, 2011. 26(6): p. 365-73.

67. Chioma, O., et al., Filifactor alocis collagenase can modulate apoptosis of normal oral keratinocytes. Mol Oral Microbiol, 2017. 32(2): p. 166-177.

68. Marchesan, J., et al., TLR4, NOD1 and NOD2 mediate immune recognition of putative newly identified periodontal pathogens. Mol Oral Microbiol, 2016. 31(3): p. 243-258.

69. Nokhbehsaim, M., et al., Regulation of matrix metalloproteinase-1 by Filifactor alocis in human gingival and monocytic cells. Clin Oral Investig, 2019.

70. Gu, Z., et al., Marijuana-Derived Cannabinoids Trigger a CB2/PI3K Axis of Suppression of the Innate Response to Oral Pathogens. Front Immunol, 2019. 10: p. 2288.

71. Han, Y.W. and X. Wang, Mobile microbiome: oral bacteria in extra-oral infections and inflammation. J Dent Res, 2013. 92(6): p. 485-91.

72. Ishihara, K., New approach for studying mobile genes using metagenomic analysis. Oral Dis, 2018. 24(4): p. 494-496.

73. Wang, Q., et al., Filifactor alocis infection and inflammatory responses in the mouse subcutaneous chamber model. Infect Immun, 2014. 82(3): p. 1205-12.

74. Gray, R.M. and M. Vidwans, Mixed anaerobic thoracic empyema: the first report of Filifactor alocis causing extra-oral disease. New Microbes New Infect, 2019. 29: p. 100528.

75. Hishiya, N., et al., Filifactor alocis brain abscess identified by $16 \mathrm{~S}$ ribosomal RNA gene sequencing: A case report. J Infect Chemother, 2020. 26(2): p. 305-307.

76. Summers, C., et al., Neutrophil kinetics in health and disease. Trends Immunol, 2010. 31(8): p. 318-24.

77. Delima, A.J. and T.E. Van Dyke, Origin and function of the cellular components in gingival crevice fluid. Periodontol 2000, 2003. 31: p. 55-76.

78. Kannengiesser, C., et al., Molecular epidemiology of chronic granulomatous disease in a series of 80 kindreds: identification of 31 novel mutations. Hum Mutat, 2008. 29(9): p. E132-49.

79. van de Vijver, E., et al., Hematologically important mutations: leukocyte adhesion deficiency (first update). Blood Cells Mol Dis, 2012. 48(1): p. 53-61.

80. $\quad$ Nauseef, W.M. and N. Borregaard, Neutrophils at work. Nat Immunol, 2014. 15(7): p. 60211.

81. Nathan, C., Neutrophils and immunity: challenges and opportunities. Nat Rev Immunol, 2006. 6(3): p. 173-82.

82. Filippi, M.D., Neutrophil transendothelial migration: updates and new perspectives. Blood, 2019. 133(20): p. 2149-2158.

83. Schmidt, S., M. Moser, and M. Sperandio, The molecular basis of leukocyte recruitment and its deficiencies. Mol Immunol, 2013. 55(1): p. 49-58.

84. Kolaczkowska, E. and P. Kubes, Neutrophil recruitment and function in health and inflammation. Nat Rev Immunol, 2013. 13(3): p. 159-75. 
85. Reichel, C.A., et al., Gelatinases mediate neutrophil recruitment in vivo: evidence for stimulus specificity and a critical role in collagen IV remodeling. J Leukoc Biol, 2008. 83(4): p. 864-74.

86. Parent, C.A., Making all the right moves: chemotaxis in neutrophils and Dictyostelium. Curr Opin Cell Biol, 2004. 16(1): p. 4-13.

87. Majumdar, R., M. Sixt, and C.A. Parent, New paradigms in the establishment and maintenance of gradients during directed cell migration. Curr Opin Cell Biol, 2014. 30: p. 33-40.

88. Levin, R., S. Grinstein, and J. Canton, The life cycle of phagosomes: formation, maturation, and resolution. Immunol Rev, 2016. 273(1): p. 156-79.

89. Allen, L.H. and A.K. Criss, Cell intrinsic functions of neutrophils and their manipulation by pathogens. Curr Opin Immunol, 2019. 60: p. 124-129.

90. El Benna, J., et al., Activation of p38 in stimulated human neutrophils: phosphorylation of the oxidase component p47phox by p38 and ERK but not by JNK. Arch Biochem Biophys, 1996. 334(2): p. 395-400.

91. el Benna, J., L.P. Faust, and B.M. Babior, The phosphorylation of the respiratory burst oxidase component p47phox during neutrophil activation. Phosphorylation of sites recognized by protein kinase $C$ and by proline-directed kinases. J Biol Chem, 1994. 269(38): p. 23431-6.

92. Groemping, Y., et al., Molecular basis of phosphorylation-induced activation of the NADPH oxidase. Cell, 2003. 113(3): p. 343-55.

93. Nauseef, W.M., How human neutrophils kill and degrade microbes: an integrated view. Immunol Rev, 2007. 219: p. 88-102.

94. Babior, B.M., J.D. Lambeth, and W. Nauseef, The neutrophil NADPH oxidase. Arch Biochem Biophys, 2002. 397(2): p. 342-4.

95. Nauseef, W.M., Detection of superoxide anion and hydrogen peroxide production by cellular NADPH oxidases. Biochim Biophys Acta, 2014. 1840(2): p. 757-67.

96. Borregaard, N. and J.B. Cowland, Granules of the human neutrophilic polymorphonuclear leukocyte. Blood, 1997. 89(10): p. 3503-21.

97. Sengelov, H., L. Kjeldsen, and N. Borregaard, Control of exocytosis in early neutrophil activation. J Immunol, 1993. 150(4): p. 1535-43.

98. Sengelov, H., et al., Mobilization of granules and secretory vesicles during in vivo exudation of human neutrophils. J Immunol, 1995. 154(8): p. 4157-65.

99. Lominadze, G., et al., Proteomic analysis of human neutrophil granules. Mol Cell Proteomics, 2005. 4(10): p. 1503-21.

100. Borregaard, N., Neutrophils, from marrow to microbes. Immunity, 2010. 33(5): p. 657-70.

101. Borregaard, N., O.E. Sørensen, and K. Theilgaard-Mönch, Neutrophil granules: a library of innate immunity proteins. Trends in Immunology, 2007. 28(8): p. 340-345.

102. Nauseef, W.M. and N. Borregaard, Neutrophils at work. Nat Immunol, 2014. 15(7): p. 602611.

103. Uriarte, S.M., et al., Comparison of Proteins Expressed on Secretory Vesicle Membranes and Plasma Membranes of Human Neutrophils. The Journal of Immunology, 2008. 180(8): p. 5575-5581.

104. Lominadze, G., et al., Proteomic Analysis of Human Neutrophil Granules. Molecular \& Cellular Proteomics, 2005. 4(10): p. 1503-1521.

105. Rørvig, S., et al., Proteome profiling of human neutrophil granule subsets, secretory vesicles, and cell membrane: correlation with transcriptome profiling of neutrophil precursors. Journal of Leukocyte Biology, 2013. 94(4): p. 711-721.

106. Saverymuttu, S.H., et al., The kinetics of 111indium distribution following injection of 111 indium labelled autologous granulocytes in man. Br J Haematol, 1985. 61(4): p. 67585. 
107. Kennedy, A.D. and F.R. DeLeo, Neutrophil apoptosis and the resolution of infection. Immunol Res, 2009. 43(1-3): p. 25-61.

108. Kobayashi, S.D., et al., Global changes in gene expression by human polymorphonuclear leukocytes during receptor-mediated phagocytosis: cell fate is regulated at the level of gene expression. Proc Natl Acad Sci U S A, 2002. 99(10): p. 6901-6.

109. Kobayashi, S.D., et al., Bacterial pathogens modulate an apoptosis differentiation program in human neutrophils. Proc Natl Acad Sci U S A, 2003. 100(19): p. 10948-53.

110. Fox, S., et al., Neutrophil apoptosis: relevance to the innate immune response and inflammatory disease. J Innate Immun, 2010. 2(3): p. 216-27.

111. Kobayashi, S.D., et al., Down-regulation of proinflammatory capacity during apoptosis in human polymorphonuclear leukocytes. J Immunol, 2003. 170(6): p. 3357-68.

112. Kobayashi, S.D., et al., An apoptosis-differentiation program in human polymorphonuclear leukocytes facilitates resolution of inflammation. J Leukoc Biol, 2003. 73(2): p. 315-22.

113. Fadok, V.A., et al., Macrophages that have ingested apoptotic cells in vitro inhibit proinflammatory cytokine production through autocrine/paracrine mechanisms involving TGF-beta, PGE2, and PAF. J Clin Invest, 1998. 101(4): p. 890-8.

114. Voll, R.E., et al., Immunosuppressive effects of apoptotic cells. Nature, 1997. 390(6658): p. 350-1.

115. Korns, D., et al., Modulation of macrophage efferocytosis in inflammation. Front Immunol, 2011. 2: p. 57.

116. Havens, A.M., et al., Stromal-derived factor-lalpha (CXCL12) levels increase in periodontal disease. J Periodontol, 2008. 79(5): p. 845-53.

117. Rijkschroeff, P., et al., Oral polymorphonuclear neutrophil characteristics in relation to oral health: a cross-sectional, observational clinical study. Int J Oral Sci, 2016. 8(3): p. 191-8.

118. Zadeh, H.H., F.C. Nichols, and K.T. Miyasaki, The role of the cell-mediated immune response to Actinobacillus actinomycetemcomitans and Porphyromonas gingivalis in periodontitis. Periodontol 2000, 1999. 20: p. 239-88.

119. Miyasaki, K.T., The neutrophil: mechanisms of controlling periodontal bacteria. J Periodontol, 1991. 62(12): p. 761-74.

120. Darveau, R.P., Periodontitis: a polymicrobial disruption of host homeostasis. Nat Rev Microbiol, 2010. 8(7): p. 481-90.

121. Uriarte, S.M., J.S. Edmisson, and E. Jimenez-Flores, Human neutrophils and oral microbiota: a constant tug-of-war between a harmonious and a discordant coexistence. 2016. 273(1): p. 282-298.

122. Hajishengallis, G., et al., Immune and regulatory functions of neutrophils in inflammatory bone loss. Semin Immunol, 2016. 28(2): p. 146-58.

123. Silva, L.M., L. Brenchley, and N.M. Moutsopoulos, Primary immunodeficiencies reveal the essential role of tissue neutrophils in periodontitis. Immunol Rev, 2019. 287(1): p. 226235.

124. Lee, W., et al., Evidence of a direct relationship between neutrophil collagenase activity and periodontal tissue destruction in vivo: role of active enzyme in human periodontitis. $\mathrm{J}$ Periodontal Res, 1995. 30(1): p. 23-33.

125. Hernandez, M., et al., Associations between matrix metalloproteinase- 8 and -14 and myeloperoxidase in gingival crevicular fluid from subjects with progressive chronic periodontitis: a longitudinal study. J Periodontol, 2010. 81(11): p. 1644-52.

126. Sochalska, M. and J. Potempa, Manipulation of Neutrophils by Porphyromonas gingivalis in the Development of Periodontitis. Front Cell Infect Microbiol, 2017. 7: p. 197.

127. Miralda, I., S.M. Uriarte, and K.R. McLeish, Multiple Phenotypic Changes Define Neutrophil Priming. Front Cell Infect Microbiol, 2017. 7: p. 217. 
128. Guentsch, A., et al., Neutrophils in chronic and aggressive periodontitis in interaction with Porphyromonas gingivalis and Aggregatibacter actinomycetemcomitans. J Periodontal Res, 2009. 44(3): p. 368-77.

129. Allen, E.M., et al., Oxidative and inflammatory status in Type 2 diabetes patients with periodontitis. J Clin Periodontol, 2011. 38(10): p. 894-901.

130. Dias, I.H., et al., Activation of the neutrophil respiratory burst by plasma from periodontitis patients is mediated by pro-inflammatory cytokines. J Clin Periodontol, 2011. 38(1): p. 17.

131. Ling, M.R., I.L. Chapple, and J.B. Matthews, Peripheral blood neutrophil cytokine hyperreactivity in chronic periodontitis. Innate Immun, 2015. 21(7): p. 714-25.

132. Ryder, M.I., Comparison of neutrophil functions in aggressive and chronic periodontitis. Periodontol 2000, 2010. 53: p. 124-37.

133. Eskan, M.A., et al., The leukocyte integrin antagonist Del-1 inhibits IL-17-mediated inflammatory bone loss. Nat Immunol, 2012. 13(5): p. 465-73.

134. Chapple, I.L. and J.B. Matthews, The role of reactive oxygen and antioxidant species in periodontal tissue destruction. Periodontol 2000, 2007. 43: p. 160-232.

135. Fine, N., et al., Distinct Oral Neutrophil Subsets Define Health and Periodontal Disease States. J Dent Res, 2016. 95(8): p. 931-8.

136. Bender, J.S., H. Thang, and M. Glogauer, Novel rinse assay for the quantification of oral neutrophils and the monitoring of chronic periodontal disease. J Periodontal Res, 2006. 41(3): p. 214-20.

137. Landzberg, M., et al., Quantifying oral inflammatory load: oral neutrophil counts in periodontal health and disease. J Periodontal Res, 2015. 50(3): p. 330-6.

138. Roberts, H.M., et al., Impaired neutrophil directional chemotactic accuracy in chronic periodontitis patients. J Clin Periodontol, 2015. 42(1): p. 1-11.

139. Jo, A.R., et al., Mechanisms of IL-8 suppression by Treponema denticola in gingival epithelial cells. Immunol Cell Biol, 2014. 92(2): p. 139-47.

140. Brissette, C.A., et al., Treponema denticola does not induce production of common innate immune mediators from primary gingival epithelial cells. Oral Microbiol Immunol, 2008. 23(6): p. 474-81.

141. Darveau, R.P., et al., Local chemokine paralysis, a novel pathogenic mechanism for Porphyromonas gingivalis. Infect Immun, 1998. 66(4): p. 1660-5.

142. Van Dyke, T.E., et al., Inhibition of neutrophil chemotaxis by soluble bacterial products. J Periodontol, 1982. 53(8): p. 502-8.

143. Ashkenazi, M., R.R. White, and D.K. Dennison, Neutrophil modulation by Actinobacillus actinomycetemcomitans. I. Chemotaxis, surface receptor expression and F-actin polymerization. J Periodontal Res, 1992. 27(4 Pt 1): p. 264-73.

144. Jones, M.M., S.T. Vanyo, and M.B. Visser, The Msp Protein of Treponema denticola Interrupts Activity of Phosphoinositide Processing in Neutrophils. Infect Immun, 2019. 87(11).

145. Magalhaes, M.A., et al., The major outer sheath protein of Treponema denticola selectively inhibits Racl activation in murine neutrophils. Cell Microbiol, 2008. 10(2): p. 344-54.

146. Jones, M.M., S.T. Vanyo, and M.B. Visser, The $C$-terminal region of the major outer sheath protein of Treponema denticola inhibits neutrophil chemotaxis. Mol Oral Microbiol, 2017. 32(5): p. 375-389.

147. Visser, M.B., et al., Treponema denticola major outer sheath protein impairs the cellular phosphoinositide balance that regulates neutrophil chemotaxis. PLoS One, 2013. 8(6): p. e66209.

148. Puthengady Thomas, B., et al., Modulation of human neutrophil functions in vitro by Treponema denticola major outer sheath protein. Infect Immun, 2006. 74(3): p. 1954-7. 
149. Darveau, R.P., et al., Ability of bacteria associated with chronic inflammatory disease to stimulate E-selectin expression and promote neutrophil adhesion. Infect Immun, 1995. 63(4): p. 1311-7.

150. Madianos, P.N., P.N. Papapanou, and J. Sandros, Porphyromonas gingivalis infection of oral epithelium inhibits neutrophil transepithelial migration. Infect Immun, 1997. 65(10): p. 3983-90.

151. Huang, G.T., et al., Differential expression of interleukin-8 and intercellular adhesion molecule-1 by human gingival epithelial cells in response to Actinobacillus actinomycetemcomitans or Porphyromonas gingivalis infection. Oral Microbiol Immunol, 1998. 13(5): p. 301-9.

152. Huang, G.T., et al., Interleukin-8 and intercellular adhesion molecule 1 regulation in oral epithelial cells by selected periodontal bacteria: multiple effects of Porphyromonas gingivalis via antagonistic mechanisms. Infect Immun, 2001. 69(3): p. 1364-72.

153. Hirschfeld, J., et al., Effects of Aggregatibacter actinomycetemcomitans leukotoxin on neutrophil migration and extracellular trap formation. J Oral Microbiol, 2016. 8: p. 33070.

154. Jusko, M., et al., A metalloproteinase karilysin present in the majority of Tannerella forsythia isolates inhibits all pathways of the complement system. J Immunol, 2012. 188(5): p. 2338-49.

155. Jusko, M., et al., A Metalloproteinase Mirolysin of Tannerella forsythia Inhibits All Pathways of the Complement System. J Immunol, 2015. 195(5): p. 2231-40.

156. Wingrove, J.A., et al., Activation of complement components $C 3$ and $C 5$ by a cysteine proteinase (gingipain-1) from Porphyromonas (Bacteroides) gingivalis. J Biol Chem, 1992. 267(26): p. 18902-7.

157. Popadiak, K., et al., Biphasic effect of gingipains from Porphyromonas gingivalis on the human complement system. J Immunol, 2007. 178(11): p. 7242-50.

158. Maekawa, T., et al., Porphyromonas gingivalis manipulates complement and TLR signaling to uncouple bacterial clearance from inflammation and promote dysbiosis. Cell Host Microbe, 2014. 15(6): p. 768-78.

159. Makkawi, H., et al., Porphyromonas gingivalis Stimulates TLR2-PI3K Signaling to Escape Immune Clearance and Induce Bone Resorption Independently of MyD88. Front Cell Infect Microbiol, 2017. 7: p. 359.

160. Shin, J., S. Ji, and Y. Choi, Ability of oral bacteria to induce tissue-destructive molecules from human neutrophils. Oral Dis, 2008. 14(4): p. 327-34.

161. Lingaas, E., et al., Demonstration of the in vitro phagocytosis of Treponema denticola by human polymorphonuclear neutrophils. Acta Pathol Microbiol Immunol Scand B, 1983. 91(5): p. 333-7.

162. Kobayashi, T., et al., Effective in vitro clearance of Porphyromonas gingivalis by Fc alpha receptor I (CD89) on gingival crevicular neutrophils. Infect Immun, 2001 . 69(5): p. 293542.

163. Guentsch, A., et al., Cleavage of IgG1 in gingival crevicular fluid is associated with the presence of Porphyromonas gingivalis. J Periodontal Res, 2013. 48(4): p. 458-65.

164. Vincents, B., et al., Cleavage of IgG1 and IgG3 by gingipain K from Porphyromonas gingivalis may compromise host defense in progressive periodontitis. FASEB J, 2011. 25(10): p. 3741-50.

165. Nauseef, W.M., The phagocyte NOX2 NADPH oxidase in microbial killing and cell signaling. Current Opinion in Immunology, 2019. 60: p. 130-140.

166. Kanzaki, H., et al., Pathways that Regulate ROS Scavenging Enzymes, and Their Role in Defense Against Tissue Destruction in Periodontitis. Front Physiol, 2017. 8: p. 351.

167. Esterbauer, H., R.J. Schaur, and H. Zollner, Chemistry and biochemistry of 4hydroxynonenal, malonaldehyde and related aldehydes. Free Radic Biol Med, 1991. 11(1): p. 81-128. 
168. Wells, P.G., et al., Oxidative stress in developmental origins of disease: teratogenesis, neurodevelopmental deficits, and cancer. Toxicol Sci, 2009. 108(1): p. 4-18.

169. Chang, M.C., et al., Butyrate induces reactive oxygen species production and affects cell cycle progression in human gingival fibroblasts. J Periodontal Res, 2013. 48(1): p. 66-73.

170. Yu, J.Y., et al., Continuous presence of $H(2) O(2)$ induces mitochondrial-mediated, MAPKand caspase-independent growth inhibition and cytotoxicity in human gingival fibroblasts. Toxicol In Vitro, 2012. 26(4): p. 561-70.

171. Ha, H., et al., Reactive oxygen species mediate RANK signaling in osteoclasts. Exp Cell Res, 2004. 301(2): p. 119-27.

172. Matthews, J.B., et al., Hyperactivity and reactivity of peripheral blood neutrophils in chronic periodontitis. Clin Exp Immunol, 2007. 147(2): p. 255-64.

173. Yamazaki, T., et al., Surface protease of Treponema denticola hydrolyzes C3 and influences function of polymorphonuclear leukocytes. Microbes Infect, 2006. 8(7): p. 175863.

174. Moriguchi, K., et al., Energy dispersive spectroscopy-scanning transmission electron microscope observations of free radical production in human polymorphonuclear leukocytes phagocytosing non-opsonized Tannerella forsythia. Microsc Res Tech, 2017. 80(6): p. 555-562.

175. Katsuragi, H., et al., Intracellular production and extracellular release of oxygen radicals by PMNs and oxidative stress on PMNs during phagocytosis of periodontopathic bacteria. Odontology, 2003. 91(1): p. 13-8.

176. Hirschfeld, J., et al., Modulation of Neutrophil Extracellular Trap and Reactive Oxygen Species Release by Periodontal Bacteria. Infect Immun, 2017. 85(12).

177. Kurgan, S., et al., Strain-Specific Impact of Fusobacterium nucleatum on Neutrophil Function. J Periodontol, 2017. 88(4): p. 380-389.

178. Johnstone, A.M., et al., A hyperactive neutrophil phenotype in patients with refractory periodontitis. J Periodontol, 2007. 78(9): p. 1788-94.

179. Aboodi, G.M., M.B. Goldberg, and M. Glogauer, Refractory periodontitis population characterized by a hyperactive oral neutrophil phenotype. J Periodontol, 2011. 82(5): p. 726-33.

180. Nussbaum, G. and L. Shapira, How has neutrophil research improved our understanding of periodontal pathogenesis? J Clin Periodontol, 2011. 38 Suppl 11: p. 49-59.

181. Ambati, M., et al., Evaluation of oxidative stress in chronic periodontitis patients following systemic antioxidant supplementation: A clinical and biochemical study. J Nat Sci Biol Med, 2017. 8(1): p. 99-103.

182. Choi, J.I., et al., Isolation, expression, and nucleotide sequence of the sod gene from Porphyromonas gingivalis. Infect Immun, 1991. 59(4): p. 1564-6.

183. Diaz, P.I., P.S. Zilm, and A.H. Rogers, Fusobacterium nucleatum supports the growth of Porphyromonas gingivalis in oxygenated and carbon-dioxide-depleted environments. Microbiology, 2002. 148(Pt 2): p. 467-472.

184. Balashova, N.V., et al., Interaction between leukotoxin and $\mathrm{Cu}, \mathrm{Zn}$ superoxide dismutase in Aggregatibacter actinomycetemcomitans. Infect Immun, 2007. 75(9): p. 4490-7.

185. Diaz, P.I., P.S. Zilm, and A.H. Rogers, The response to oxidative stress of Fusobacterium nucleatum grown in continuous culture. FEMS Microbiol Lett, 2000. 187(1): p. 31-4.

186. Sztukowska, M., et al., Role of rubrerythrin in the oxidative stress response of Porphyromonas gingivalis. Mol Microbiol, 2002. 44(2): p. 479-88.

187. Johnson, N.A., Y. Liu, and H.M. Fletcher, Alkyl hydroperoxide peroxidase subunit C $(a h p C)$ protects against organic peroxides but does not affect the virulence of Porphyromonas gingivalis W83. Oral Microbiol Immunol, 2004. 19(4): p. 233-9.

188. Diaz, P.I., et al., Studies on NADH oxidase and alkyl hydroperoxide reductase produced by Porphyromonas gingivalis. Oral Microbiol Immunol, 2004. 19(3): p. 137-43. 
189. Smalley, J.W. and T. Olczak, Heme acquisition mechanisms of Porphyromonas gingivalis - strategies used in a polymicrobial community in a heme-limited host environment. Mol Oral Microbiol, 2017. 32(1): p. 1-23.

190. Rangarajan, M., et al., Hemin binding by Porphyromonas gingivalis strains is dependent on the presence of A-LPS. Mol Oral Microbiol, 2017. 32(5): p. 365-374.

191. Diamond, G., N. Beckloff, and L.K. Ryan, Host defense peptides in the oral cavity and the lung: similarities and differences. J Dent Res, 2008. 87(10): p. 915-27.

192. Puklo, M., et al., Analysis of neutrophil-derived antimicrobial peptides in gingival crevicular fluid suggests importance of cathelicidin LL-37 in the innate immune response against periodontogenic bacteria. Oral Microbiol Immunol, 2008. 23(4): p. 328-35.

193. Turkoglu, O., et al., Gingival crevicular fluid levels of cathelicidin LL-37 and interleukin18 in patients with chronic periodontitis. J Periodontol, 2009. 80(6): p. 969-76.

194. Lee, S.H., et al., Antibacterial and lipopolysaccharide (LPS)-neutralising activity of human cationic antimicrobial peptides against periodontopathogens. Int J Antimicrob Agents, 2010. 35(2): p. 138-45.

195. Musrati, A.A., et al., Morphological and functional adaptations of Fusobacterium nucleatum exposed to human neutrophil Peptide-1. Anaerobe, 2016. 39: p. 31-8.

196. Ding, Y., et al., Release and activation of human neutrophil matrix metallo- and serine proteinases during phagocytosis of Fusobacterium nucleatum, Porphyromonas gingivalis and Treponema denticola. J Clin Periodontol, 1997. 24(4): p. 237-48.

197. Sheikhi, M., A. Gustafsson, and C. Jarstrand, Cytokine, elastase and oxygen radical release by Fusobacterium nucleatum-activated leukocytes: a possible pathogenic factor in periodontitis. J Clin Periodontol, 2000. 27(10): p. 758-62.

198. Ji, S., et al., Susceptibility of various oral bacteria to antimicrobial peptides and to phagocytosis by neutrophils. J Periodontal Res, 2007. 42(5): p. 410-9.

199. Koziel, J., et al., Proteolytic inactivation of LL-37 by karilysin, a novel virulence mechanism of Tannerella forsythia. J Innate Immun, 2010. 2(3): p. 288-93.

200. Ksiazek, M., et al., Miropin, a novel bacterial serpin from the periodontopathogen Tannerella forsythia, inhibits a broad range of proteases by using different peptide bonds within the reactive center loop. J Biol Chem, 2015. 290(1): p. 658-70.

201. Carlisle, M.D., R.N. Srikantha, and K.A. Brogden, Degradation of human alpha- and betadefensins by culture supernatants of Porphyromonas gingivalis strain 381. J Innate Immun, 2009. 1(2): p. 118-22.

202. Gutner, M., et al., Saliva enables the antimicrobial activity of LL-37 in the presence of proteases of Porphyromonas gingivalis. Infect Immun, 2009. 77(12): p. 5558-63.

203. Kuula, H., et al., Human beta-defensin-1 and -2 and matrix metalloproteinase-25 and -26 expression in chronic and aggressive periodontitis and in peri-implantitis. Arch Oral Biol, 2008. 53(2): p. 175-86.

204. Odell, E.W. and P.J. Wu, Susceptibility of Porphyromonas gingivalis and P. asaccharolytica to the non-oxidative killing mechanisms of human neutrophils. Arch Oral Biol, 1992. 37(8): p. 597-601.

205. Shelburne, C.E., et al., Induction of \{beta\}-defensin resistance in the oral anaerobe Porphyromonas gingivalis. Antimicrob Agents Chemother, 2005. 49(1): p. 183-7.

206. Sela, M.N., Role of Treponema denticola in periodontal diseases. Crit Rev Oral Biol Med, 2001. 12(5): p. 399-413.

207. Sela, M.N., et al., Lipoproteins of Treponema denticola: their effect on human polymorphonuclear neutrophils. J Periodontal Res, 1997. 32(5): p. 455-66.

208. Gursoy, U.K., et al., Molecular forms and fragments of salivary MMP-8 in relation to periodontitis. J Clin Periodontol, 2018. 45(12): p. 1421-1428. 
209. Claesson, R., et al., Release and activation of matrix metalloproteinase 8 from human neutrophils triggered by the leukotoxin of Actinobacillus actinomycetemcomitans. J Periodontal Res, 2002. 37(5): p. 353-9.

210. Nicu, E.A., et al., Characterization of oral polymorphonuclear neutrophils in periodontitis patients: a case-control study. BMC Oral Health, 2018. 18(1): p. 149.

211. Ujiie, Y., et al., Neutrophil elastase is involved in the initial destruction of human periodontal ligament. J Periodontal Res, 2007. 42(4): p. 325-30.

212. Karim, A.Y., et al., A novel matrix metalloprotease-like enzyme (karilysin) of the periodontal pathogen Tannerella forsythia ATCC 43037. Biol Chem, 2010. 391(1): p. 10517.

213. Williams, S.E., et al., SLPI and elafin: one glove, many fingers. Clin Sci (Lond), 2006. 110(1): p. 21-35.

214. Yin, L., et al., Differential effects of periopathogens on host protease inhibitors SLPI, elafin, SCCA1, and SCCA2. J Oral Microbiol, 2010. 2.

215. Into, T., et al., Arginine-specific gingipains from Porphyromonas gingivalis deprive protective functions of secretory leucocyte protease inhibitor in periodontal tissue. Clin Exp Immunol, 2006. 145(3): p. 545-54.

216. Laugisch, O., et al., Periodontal pathogens affect the level of protease inhibitors in gingival crevicular fluid. Mol Oral Microbiol, 2012. 27(1): p. 45-56.

217. Sollberger, G., D.O. Tilley, and A. Zychlinsky, Neutrophil Extracellular Traps: The Biology of Chromatin Externalization. Developmental Cell, 2018. 44(5): p. 542-553.

218. White, P.C., et al., Neutrophil Extracellular Traps in Periodontitis: A Web of Intrigue. J Dent Res, 2016. 95(1): p. 26-34.

219. Vitkov, L., et al., Extracellular neutrophil traps in periodontitis. J Periodontal Res, 2009. 44(5): p. 664-72.

220. Hirschfeld, J., et al., Neutrophil extracellular trap formation in supragingival biofilms. Int J Med Microbiol, 2015. 305(4-5): p. 453-63.

221. Alyami, H.M., et al., Role of NOD1/NOD2 receptors in Fusobacterium nucleatum mediated NETosis. Microb Pathog, 2019. 131: p. 53-64.

222. Palmer, L.J., et al., Influence of complement on neutrophil extracellular trap release induced by bacteria. J Periodontal Res, 2016. 51(1): p. 70-6.

223. Jayaprakash, K., et al., The role of phagocytosis, oxidative burst and neutrophil extracellular traps in the interaction between neutrophils and the periodontal pathogen Porphyromonas gingivalis. Mol Oral Microbiol, 2015. 30(5): p. 361-75.

224. Bryzek, D., et al., Triggering NETosis via protease-activated receptor (PAR)-2 signaling as a mechanism of hijacking neutrophils function for pathogen benefits. PLoS Pathog, 2019. 15(5): p. e1007773.

225. Palmer, L.J., et al., Extracellular deoxyribonuclease production by periodontal bacteria. J Periodontal Res, 2012. 47(4): p. 439-45.

226. Doke, M., et al., Nucleases from Prevotella intermedia can degrade neutrophil extracellular traps. Mol Oral Microbiol, 2017. 32(4): p. 288-300.

227. Aliko, A., et al., Discovery of Novel Potential Reversible Peptidyl Arginine Deiminase Inhibitor. Int J Mol Sci, 2019. 20(9).

228. Vitkov, L., et al., Periodontal sources of citrullinated antigens and TLR agonists related to RA. Autoimmunity, 2018. 51(6): p. 304-309.

229. Stobernack, T., et al., A Secreted Bacterial Peptidylarginine Deiminase Can Neutralize Human Innate Immune Defenses. mBio, 2018. 9(5).

230. Cooper, P.R., L.J. Palmer, and I.L. Chapple, Neutrophil extracellular traps as a new paradigm in innate immunity: friend or foe? Periodontol 2000, 2013. 63(1): p. 165-97.

231. Eick, S., et al., Phagocytosis of periodontopathogenic bacteria by crevicular granulocytes is depressed in progressive periodontitis. Infection, 2000. 28(5): p. 301-4. 
232. Yoneda, M., K. Maeda, and M. Aono, Suppression of bactericidal activity of human polymorphonuclear leukocytes by Bacteroides gingivalis. Infect Immun, 1990. 58(2): p. 406-11.

233. Kadowaki, T., et al., Suppression of pathogenicity of Porphyromonas gingivalis by newly developed gingipain inhibitors. Mol Pharmacol, 2004. 66(6): p. 1599-606.

234. Mangan, D.F., et al., Lectinlike interactions of Fusobacterium nucleatum with human neutrophils. Infect Immun, 1989. 57(11): p. 3601-11.

235. Lai, P.C., M.R. Schibler, and J.D. Walters, Azithromycin enhances phagocytic killing of Aggregatibacter actinomycetemcomitans Y4 by human neutrophils. J Periodontol, 2015. 86(1): p. 155-61.

236. Permpanich, P., M.J. Kowolik, and D.M. Galli, Resistance of fluorescent-labelled Actinobacillus actinomycetemcomitans strains to phagocytosis and killing by human neutrophils. Cell Microbiol, 2006. 8(1): p. 72-84.

237. Pabst, M.J., et al., Inhibition of neutrophil and monocyte defensive functions by nicotine. $\mathrm{J}$ Periodontol, 1995. 66(12): p. 1047-55.

238. Gaibani, P., et al., Killing of Treponema denticola by mouse peritoneal macrophages. J Dent Res, 2010. 89(5): p. 521-6.

239. Jung, Y.J., H.K. Jun, and B.K. Choi, Gingipain-dependent augmentation by Porphyromonas gingivalis of phagocytosis of Tannerella forsythia. Mol Oral Microbiol, 2016. 31(6): p. 457-471.

240. Rotstein, O.D., et al., Succinic acid, a metabolic by-product of Bacteroides species, inhibits polymorphonuclear leukocyte function. Infect Immun, 1985. 48(2): p. 402-8.

241. Rotstein, O.D., P.E. Nasmith, and S. Grinstein, The Bacteroides by-product succinic acid inhibits neutrophil respiratory burst by reducing intracellular pH. Infect Immun, 1987. 55(4): p. 864-70.

242. Eftimiadi, C., et al., Short-chain fatty acids produced by anaerobic bacteria alter the physiological responses of human neutrophils to chemotactic peptide. J Infect, 1987. 14(1): p. 43-53.

243. Hajishengallis, G., New developments in neutrophil biology and periodontitis. Periodontol 2000, 2020. 82(1): p. 78-92.

244. Burns, E., et al., TLR2-dependent inflammatory response to Porphyromonas gingivalis is MyD88 independent, whereas MyD88 is required to clear infection. J Immunol, 2010. 184(3): p. 1455-62.

245. Tamassia, N., et al., Cytokine production by human neutrophils: Revisiting the "dark side of the moon". European Journal of Clinical Investigation, 2018. 48(S2): p. e12952.

246. Cassatella, M.A., Neutrophil-Derived Proteins: Selling Cytokines by the Pound, in Advances in Immunology, F.J. Dixon, Editor. 1999, Academic Press. p. 369-509.

247. Tecchio, C. and M.A. Cassatella, Neutrophil-derived chemokines on the road to immunity. Seminars in Immunology, 2016. 28(2): p. 119-128.

248. Scapini, P. and M.A. Cassatella, Social networking of human neutrophils within the immune system. Blood, 2014. 124(5): p. 710-719.

249. Cassatella, M.A., et al., Biological Roles of Neutrophil-Derived Granule Proteins and Cytokines. Trends Immunol, 2019. 40(7): p. 648-664.

250. Tamassia, N., et al., Cytokine production by human neutrophils: Revisiting the "dark side of the moon". Eur J Clin Invest, 2018. 48 Suppl 2: p. e12952.

251. Polak, D., et al., Virulence mechanism of bacteria in mixed infection: attenuation of cytokine levels and evasion of polymorphonuclear leukocyte phagocytosis. J Periodontol, 2013. 84(10): p. 1463-8.

252. Yoshimura, A., et al., Secretion of IL-1 beta, TNF-alpha, IL-8 and IL-1ra by human polymorphonuclear leukocytes in response to lipopolysaccharides from periodontopathic bacteria. J Periodontal Res, 1997. 32(3): p. 279-86. 
253. Vashishta, A., et al., Putative Periodontal Pathogens, Filifactor Alocis and Peptoanaerobacter Stomatis, Induce Differential Cytokine and Chemokine Production by Human Neutrophils. Pathogens, 2019. 8(2).

254. Tecchio, C., A. Micheletti, and M.A. Cassatella, Neutrophil-derived cytokines: facts beyond expression. Front Immunol, 2014. 5: p. 508.

255. Calzetti, F., et al., The importance of being "pure" neutrophils. J Allergy Clin Immunol, 2017. 139(1): p. 352-355 e6.

256. Li, J., et al., Necroptosis in the periodontal homeostasis: Signals emanating from dying cells. Oral Dis, 2018. 24(6): p. 900-907.

257. Song, B., et al., Programmed cell death in periodontitis: recent advances and future perspectives. Oral Dis, 2017. 23(5): p. 609-619.

258. Bantel, H., et al., Caspase activation is involved in chronic periodontitis. FEBS Lett, 2005. 579(25): p. 5559-64.

259. Jun, H.K., Y.J. Jung, and B.K. Choi, Treponema denticola, Porphyromonas gingivalis, and Tannerella forsythia induce cell death and release of endogenous danger signals. Arch Oral Biol, 2017. 73: p. 72-78.

260. Lakschevitz, F.S., G.M. Aboodi, and M. Glogauer, Oral neutrophil transcriptome changes result in a pro-survival phenotype in periodontal diseases. PLoS One, 2013. 8(7): p. e68983.

261. Jewett, A., et al., Induction of apoptotic cell death in peripheral blood mononuclear and polymorphonuclear cells by an oral bacterium, Fusobacterium nucleatum. Infect Immun, 2000. 68(4): p. 1893-8.

262. Coxon, A., et al., A novel role for the beta 2 integrin CD11b/CD18 in neutrophil apoptosis: a homeostatic mechanism in inflammation. Immunity, 1996. 5(6): p. 653-66.

263. Hotta, K., et al., The loss of susceptibility to apoptosis in exudated tissue neutrophils is associated with their nuclear factor-kappa B activation. Eur J Pharmacol, 2001. 433(1): p. 17-27.

264. Gamonal, J., et al., Delayed neutrophil apoptosis in chronic periodontitis patients. J Clin Periodontol, 2003. 30(7): p. 616-23.

265. Galicia, J.C., et al., Neutrophils rescue gingival epithelial cells from bacterial-induced apoptosis. J Leukoc Biol, 2009. 86(1): p. 181-6.

266. Hiroi, M., et al., Inhibition by Porphyromonas gingivalis LPS of apoptosis induction in human peripheral blood polymorphonuclear leukocytes. Anticancer Res, 1998. 18(5A): p. 3475-9.

267. Preshaw, P.M., R.E. Schifferle, and J.D. Walters, Porphyromonas gingivalis lipopolysaccharide delays human polymorphonuclear leukocyte apoptosis in vitro. J Periodontal Res, 1999. 34(4): p. 197-202.

268. Johansson, A., Aggregatibacter actinomycetemcomitans leukotoxin: a powerful tool with capacity to cause imbalance in the host inflammatory response. Toxins (Basel), 2011. 3(3): p. 242-59.

269. Edmisson, J.S., et al., Filifactor alocis modulates human neutrophil antimicrobial functional responses. Cellular Microbiology, 2018. 20(6): p. e12829.

270. Armstrong, C.L., et al., Filifactor alocis Promotes Neutrophil Degranulation and Chemotactic Activity. Infection and Immunity, 2016. 84(12): p. 3423-3433.

271. Armstrong, C.L., et al., Filifactor alocis manipulates human neutrophils affecting their ability to release neutrophil extracellular traps induced by PMA. Innate Immunity, 2018. 24(4): p. 210-220.

272. Godovikova, V., M.P. Goetting-Minesky, and J.C. Fenno, Composition and localization of Treponema denticola outer membrane complexes. Infect Immun, 2011. 79(12): p. 486875. 
273. Gosling, P.T., et al., Immunohistological analysis of Tannerella forsythia-induced lesions in a murine model. Oral Microbiol Immunol, 2005. 20(1): p. 25-30.

274. Lenzo, J.C., et al., Determination of Active Phagocytosis of Unopsonized Porphyromonas gingivalis by Macrophages and Neutrophils Using the pH-Sensitive Fluorescent Dye pHrodo. Infect Immun, 2016. 84(6): p. 1753-1760.

275. Armstrong, C.L., et al., Filifactor alocis Promotes Neutrophil Degranulation and Chemotactic Activity. Infect Immun, 2016. 84(12): p. 3423-3433.

276. Armstrong, C.L., et al., Filifactor alocis manipulates human neutrophils affecting their ability to release neutrophil extracellular traps induced by PMA. Innate Immun, 2018. 24(4): p. 210-220.

277. Jimenez Flores, E., et al., Peptoanaerobacter stomatis Primes Human Neutrophils and Induces Granule Exocytosis. Infect Immun, 2017. 85(7).

278. Siqueira, J.F., Jr. and I.N. Rocas, Detection of Filifactor alocis in endodontic infections associated with different forms of periradicular diseases. Oral Microbiol Immunol, 2003. 18(4): p. 263-5.

279. Spooner, R., et al., In Situ Anabolic Activity of Periodontal Pathogens Porphyromonas gingivalis and Filifactor alocis in Chronic Periodontitis. Sci Rep, 2016. 6: p. 33638.

280. Belstrom, D., et al., Metagenomic and metatranscriptomic analysis of saliva reveals disease-associated microbiota in patients with periodontitis and dental caries. NPJ Biofilms Microbiomes, 2017. 3: p. 23.

281. Scott, D.A. and J. Krauss, Neutrophils in periodontal inflammation. Front Oral Biol, 2012. 15: p. 56-83.

282. Uriarte, S.M., J.S. Edmisson, and E. Jimenez-Flores, Human neutrophils and oral microbiota: a constant tug-of-war between a harmonious and a discordant coexistence. Immunol Rev, 2016. 273(1): p. 282-98.

283. Amulic, B., et al., Neutrophil function: from mechanisms to disease. Annu Rev Immunol, 2012. 30: p. 459-89.

284. Edmisson, J.S., et al., Filifactor alocis modulates human neutrophil antimicrobial functional responses. Cell Microbiol, 2018. 20(6): p. e12829.

285. Jordan, J.D., E.M. Landau, and R. Iyengar, Signaling networks: the origins of cellular multitasking. Cell, 2000. 103(2): p. 193-200.

286. Kyriakis, J.M. and J. Avruch, Mammalian MAPK signal transduction pathways activated by stress and inflammation: a 10-year update. Physiol Rev, 2012. 92(2): p. 689-737.

287. Cloutier, A., et al., Differential involvement of NF-kappaB and MAP kinase pathways in the generation of inflammatory cytokines by human neutrophils. J Leukoc Biol, 2007. 81(2): p. 567-77.

288. Cloutier, A., et al., Inflammatory cytokine production by human neutrophils involves C/EBP transcription factors. J Immunol, 2009. 182(1): p. 563-71.

289. Simard, F.A., et al., MEK-independent ERK activation in human neutrophils and its impact on functional responses. J Leukoc Biol, 2015. 98(4): p. 565-73.

290. Roy, C.R. and E.S. Mocarski, Pathogen subversion of cell-intrinsic innate immunity. Nat Immunol, 2007. 8(11): p. 1179-87.

291. Arthur, J.S. and S.C. Ley, Mitogen-activated protein kinases in innate immunity. Nat Rev Immunol, 2013. 13(9): p. 679-92.

292. Kim, K.H., et al., Mycobacterium tuberculosis Eis protein initiates suppression of host immune responses by acetylation of DUSP16/MKP-7. Proc Natl Acad Sci U S A, 2012. 109(20): p. 7729-34.

293. Subrahmanyam, Y.V., et al., RNA expression patterns change dramatically in human neutrophils exposed to bacteria. Blood, 2001. 97(8): p. 2457-68. 
294. Borjesson, D.L., et al., Insights into pathogen immune evasion mechanisms: Anaplasma phagocytophilum fails to induce an apoptosis differentiation program in human neutrophils. J Immunol, 2005. 174(10): p. 6364-72.

295. Wright, H.J., et al., Fusobacterium nucleatum regulation of neutrophil transcription. J Periodontal Res, 2011. 46(1): p. 1-12.

296. Schwartz, J.T., et al., Francisella tularensis alters human neutrophil gene expression: insights into the molecular basis of delayed neutrophil apoptosis. J Innate Immun, 2013. 5(2): p. 124-36.

297. Uriarte, S.M., et al., Granule exocytosis contributes to priming and activation of the human neutrophil respiratory burst. J Immunol, 2011. 187(1): p. 391-400.

298. SenGupta, S., et al., A Pseudomonas aeruginosa hepta-acylated lipid A variant associated with cystic fibrosis selectively activates human neutrophils. Journal of Leukocyte Biology, 2016. 100(5): p. 1047-1059.

299. Babraham Bioinformatics - FastQC A Quality Control tool for High Throughput Sequence Data. 2019; Available from: https://www.bioinformatics.babraham.ac.uk/projects/fastqc/.

300. Kim, D., et al., TopHat2: accurate alignment of transcriptomes in the presence of insertions, deletions and gene fusions. Genome Biol, 2013. 14(4): p. R36.

301. Trapnell, C., et al., Differential analysis of gene regulation at transcript resolution with RNA-seq. Nat Biotechnol, 2013. 31(1): p. 46-53.

302. Trapnell, C., et al., Differential gene and transcript expression analysis of RNA-seq experiments with TopHat and Cufflinks. Nat Protoc, 2012. 7(3): p. 562-78.

303. Arruda-Silva, F., et al., Human Neutrophils Produce CCL23 in Response to Various TLRAgonists and TNFalpha. Front Cell Infect Microbiol, 2017. 7: p. 176.

304. Winkles, J.A., The TWEAK-Fn14 cytokine-receptor axis: discovery, biology and therapeutic targeting. Nat Rev Drug Discov, 2008. 7(5): p. 411-25.

305. Liu, H., et al., TWEAK/Fn14 promotes oxidative stress through AMPK/PGClalpha/MnSOD signaling pathway in endothelial cells. Mol Med Rep, 2018. 17(1): p. 1998-2004.

306. Yang, J., et al., High TNFRSF12A level associated with MMP-9 overexpression is linked to poor prognosis in breast cancer: Gene set enrichment analysis and validation in largescale cohorts. PLoS One, 2018. 13(8): p. e0202113.

307. Zhu, C., et al., TWEAK/Fn14 interaction induces proliferation and migration in human airway smooth muscle cells via activating the NF-kappaB pathway. J Cell Biochem, 2018. 119(4): p. 3528-3536.

308. Huang da, W., B.T. Sherman, and R.A. Lempicki, Bioinformatics enrichment tools: paths toward the comprehensive functional analysis of large gene lists. Nucleic Acids Res, 2009. 37(1): p. 1-13.

309. Huang da, W., B.T. Sherman, and R.A. Lempicki, Systematic and integrative analysis of large gene lists using DAVID bioinformatics resources. Nat Protoc, 2009. 4(1): p. 44-57.

310. Wu, S.Y., et al., Cell Intrinsic Galectin-3 Attenuates Neutrophil ROS-Dependent Killing of Candida by Modulating CR3 Downstream Syk Activation. Front Immunol, 2017. 8: p. 48.

311. Konkel, J.E., C. O'Boyle, and S. Krishnan, Distal Consequences of Oral Inflammation. Front Immunol, 2019. 10: p. 1403.

312. Nahas, N., et al., Tyrosine phosphorylation and activation of a new mitogen-activated protein (MAP)-kinase cascade in human neutrophils stimulated with various agonists. Biochem J, 1996. 318 ( Pt 1): p. 247-53.

313. McLeish, K.R., et al., Activation of mitogen-activated protein kinase cascades during priming of human neutrophils by TNF-alpha and GM-CSF. J Leukoc Biol, 1998. 64(4): p. 537-45.

314. Graves, D.T. and D. Cochran, The contribution of interleukin-1 and tumor necrosis factor to periodontal tissue destruction. J Periodontol, 2003. 74(3): p. 391-401. 
315. Singh, P., et al., Salivary TNF-alpha: A potential marker of periodontal destruction. J Indian Soc Periodontol, 2014. 18(3): p. 306-10.

316. Fortin, C.F., et al., A class IA PI3K controls inflammatory cytokine production in human neutrophils. Eur J Immunol, 2011. 41(6): p. 1709-19.

317. Cassatella, M.A., Neutrophil-derived proteins: selling cytokines by the pound. Adv Immunol, 1999. 73: p. 369-509.

318. Newburger, P.E., Y.V. Subrahmanyam, and S.M. Weissman, Global analysis of neutrophil gene expression. Curr Opin Hematol, 2000. 7(1): p. 16-20.

319. Kobayashi, S.D. and F.R. DeLeo, Role of neutrophils in innate immunity: a systems biology-level approach. Wiley Interdiscip Rev Syst Biol Med, 2009. 1(3): p. 309-333.

320. Rungelrath, V., S.D. Kobayashi, and F.R. DeLeo, Neutrophils in innate immunity and systems biology-level approaches. Wiley Interdiscip Rev Syst Biol Med, 2020. 12(1): p. e1458.

321. Nauseef, W.M., The phagocyte NOX2 NADPH oxidase in microbial killing and cell signaling. Curr Opin Immunol, 2019. 60: p. 130-140.

322. Johnson, M.B., et al., Opa+ Neisseria gonorrhoeae exhibits reduced survival in human neutrophils via Src family kinase-mediated bacterial trafficking into mature phagolysosomes. Cell Microbiol, 2015. 17(5): p. 648-65.

323. Taheri, N., A. Fahlgren, and M. Fallman, Yersinia pseudotuberculosis Blocks Neutrophil Degranulation. Infect Immun, 2016. 84(12): p. 3369-3378.

324. Kilpatrick, L.E., et al., Regulation of TNF mediated antiapoptotic signaling in human neutrophils: role of delta-PKC and ERK1/2. J Leukoc Biol, 2006. 80(6): p. 1512-21.

325. Futosi, K., S. Fodor, and A. Mocsai, Neutrophil cell surface receptors and their intracellular signal transduction pathways. Int Immunopharmacol, 2013. 17(3): p. 638-50.

326. Satoh, T. and S. Akira, Toll-Like Receptor Signaling and Its Inducible Proteins. Microbiol Spectr, 2016. 4(6).

327. Hayashi, F., T.K. Means, and A.D. Luster, Toll-like receptors stimulate human neutrophil function. Blood, 2003. 102(7): p. 2660-9.

328. Janke, M., et al., Selective and direct activation of human neutrophils but not eosinophils by Toll-like receptor 8. J Allergy Clin Immunol, 2009. 123(5): p. 1026-33.

329. Berger, M., et al., Neutrophils express distinct RNA receptors in a non-canonical way. $\mathrm{J}$ Biol Chem, 2012. 287(23): p. 19409-17.

330. Zimmermann, M., et al., Chromatin remodelling and autocrine TNFalpha are required for optimal interleukin-6 expression in activated human neutrophils. Nat Commun, 2015. 6: p. 6061.

331. Kawai, T. and S. Akira, Signaling to NF-kappaB by Toll-like receptors. Trends Mol Med, 2007. 13(11): p. 460-9.

332. Sabroe, I., et al., Toll-like receptor (TLR)2 and TLR4 in human peripheral blood granulocytes: a critical role for monocytes in leukocyte lipopolysaccharide responses. $\mathrm{J}$ Immunol, 2002. 168(9): p. 4701-10.

333. Lin, S.C., Y.C. Lo, and H. Wu, Helical assembly in the MyD88-IRAK4-IRAK2 complex in TLR/IL-1R signalling. Nature, 2010. 465(7300): p. 885-90.

334. Balka, K.R. and D. De Nardo, Understanding early TLR signaling through the Myddosome. J Leukoc Biol, 2019. 105(2): p. 339-351.

335. Takaoka, A., et al., Integral role of IRF-5 in the gene induction programme activated by Toll-like receptors. Nature, 2005. 434(7030): p. 243-9.

336. Sabroe, I., et al., Selective roles for Toll-like receptor (TLR)2 and TLR4 in the regulation of neutrophil activation and life span. J Immunol, 2003. 170(10): p. 5268-75.

337. Strassheim, D., et al., Phosphoinositide 3-kinase and Akt occupy central roles in inflammatory responses of Toll-like receptor 2-stimulated neutrophils. J Immunol, 2004. 172(9): p. 5727-33. 
338. Aliprantis, A.O., et al., The apoptotic signaling pathway activated by Toll-like receptor-2. EMBO J, 2000. 19(13): p. 3325-36.

339. Sabroe, I., S.K. Dower, and M.K. Whyte, The role of Toll-like receptors in the regulation of neutrophil migration, activation, and apoptosis. Clin Infect Dis, 2005. 41 Suppl 7: p. S421-6.

340. Hirschfeld, M., et al., Cutting edge: repurification of lipopolysaccharide eliminates signaling through both human and murine toll-like receptor 2. J Immunol, 2000. 165(2): p. 618-22.

341. Hajjar, A.M., et al., Cutting edge: functional interactions between toll-like receptor (TLR) 2 and TLR1 or TLR6 in response to phenol-soluble modulin. J Immunol, 2001. 166(1): p. 15-9.

342. Whitmore, L.C., et al., A Common Genetic Variant in TLRI Enhances Human Neutrophil Priming and Impacts Length of Intensive Care Stay in Pediatric Sepsis. J Immunol, 2016. 196(3): p. 1376-86.

343. Wilde, I., et al., Direct stimulatory effects of the TLR2/6 ligand bacterial lipopeptide MALP-2 on neutrophil granulocytes. Med Microbiol Immunol, 2007. 196(2): p. 61-71.

344. McGuire, V.A. and J.S. Arthur, Subverting Toll-Like Receptor Signaling by Bacterial Pathogens. Front Immunol, 2015. 6: p. 607.

345. Mocsai, A., et al., G-protein-coupled receptor signaling in Syk-deficient neutrophils and mast cells. Blood, 2003. 101(10): p. 4155-63.

346. Mocsai, A., et al., Adhesion-dependent degranulation of neutrophils requires the Src family kinases Fgr and Hck. J Immunol, 1999. 162(2): p. 1120-6.

347. Yu, Z., T. Chen, and X. Cao, Neutrophil sensing of cytoplasmic, pathogenic DNA in a cGAS-STING-independent manner. Cell Mol Immunol, 2016. 13(4): p. 411-4.

348. Jyoti, A., et al., Interaction of inducible nitric oxide synthase with rac2 regulates reactive oxygen and nitrogen species generation in the human neutrophil phagosomes: implication in microbial killing. Antioxid Redox Signal, 2014. 20(3): p. 417-31.

349. Evans, T.J., et al., Cytokine-treated human neutrophils contain inducible nitric oxide synthase that produces nitration of ingested bacteria. Proc Natl Acad Sci U S A, 1996. 93(18): p. 9553-8.

350. Webb, J.L., J.M. Polak, and T.J. Evans, Effect of adhesion on inducible nitric oxide synthase (iNOS) production in purified human neutrophils. Clin Exp Immunol, 2001. 123(1): p. 42-8.

351. Saini, R. and S. Singh, Inducible nitric oxide synthase: An asset to neutrophils. J Leukoc Biol, 2019. 105(1): p. 49-61.

352. Miralda, I., S.M. Uriarte, and K.R. McLeish, Multiple Phenotypic Changes Define Neutrophil Priming. Frontiers in Cellular and Infection Microbiology, 2017. 7.

353. Erbeck, K., J.B. Klein, and K.R. McLeish, Differential uncoupling of chemoattractant receptors from $G$ proteins in retinoic acid-differentiated HL-60 granulocytes. J Immunol, 1993. 150(5): p. 1913-21.

354. Underhill, D.M., Macrophage recognition of zymosan particles. J Endotoxin Res, 2003. 9(3): p. 176-80.

355. Lawrence, T., The nuclear factor NF-kappaB pathway in inflammation. Cold Spring Harb Perspect Biol, 2009. 1(6): p. a001651.

356. De Nardo, D., Toll-like receptors: Activation, signalling and transcriptional modulation. Cytokine, 2015. 74(2): p. 181-9.

357. Hajishengallis, G., et al., Immune and regulatory functions of neutrophils in inflammatory bone loss. Seminars in Immunology, 2016. 28(2): p. 146-158.

358. Prince, L.R., et al., The role of TLRs in neutrophil activation. Curr Opin Pharmacol, 2011. 11(4): p. 397-403. 
359. Fang, L., et al., TLR2 mediates phagocytosis and autophagy through JNK signaling pathway in Staphylococcus aureus-stimulated RAW264.7 cells. Cell Signal, 2014. 26(4): p. 806-14.

360. Shen, Y., et al., Toll-like receptor 2- and MyD88-dependent phosphatidylinositol 3-kinase and Racl activation facilitates the phagocytosis of Listeria monocytogenes by murine macrophages. Infect Immun, 2010. 78(6): p. 2857-67.

361. Acorci-Valerio, M.J., et al., Role of TLR2 and TLR4 in human neutrophil functions against Paracoccidioides brasiliensis. Scand J Immunol, 2010. 71(2): p. 99-108.

362. Behera, A.K., et al., MyD88 deficiency results in tissue-specific changes in cytokine induction and inflammation in interleukin-18-independent mice infected with Borrelia burgdorferi. Infect Immun, 2006. 74(3): p. 1462-70.

363. Behera, A.K., et al., Identification of a TLR-independent pathway for Borrelia burgdorferiinduced expression of matrix metalloproteinases and inflammatory mediators through binding to integrin alpha 3 beta 1. J Immunol, 2006. 177(1): p. 657-64.

364. Bolz, D.D., et al., MyD88 plays a unique role in host defense but not arthritis development in Lyme disease. J Immunol, 2004. 173(3): p. 2003-10.

365. Liu, N., et al., Myeloid differentiation antigen 88 deficiency impairs pathogen clearance but does not alter inflammation in Borrelia burgdorferi-infected mice. Infect Immun, 2004. 72(6): p. 3195-203.

366. Blander, J.M. and R. Medzhitov, Regulation of phagosome maturation by signals from tolllike receptors. Science, 2004. 304(5673): p. 1014-8.

367. Henneke, P., et al., Cellular activation, phagocytosis, and bactericidal activity against group B streptococcus involve parallel myeloid differentiation factor 88-dependent and independent signaling pathways. J Immunol, 2002. 169(7): p. 3970-7.

368. Marr, K.A., et al., Differential role of MyD88 in macrophage-mediated responses to opportunistic fungal pathogens. Infect Immun, 2003. 71(9): p. 5280-6.

369. Andrews, K., et al., TLR2 regulates neutrophil recruitment and cytokine production with minor contributions from TLR9 during hypersensitivity pneumonitis. PLoS One, 2013. 8(8): p. e73143.

370. Duggan, J.M., et al., Synergistic interactions of TLR2/6 and TLR9 induce a high level of resistance to lung infection in mice. J Immunol, 2011. 186(10): p. 5916-26.

371. Zhang, Q., et al., Circulating mitochondrial DAMPs cause inflammatory responses to injury. Nature, 2010. 464(7285): p. 104-7.

372. van Bruggen, R., et al., Toll-like receptor responses in IRAK-4-deficient neutrophils. J Innate Immun, 2010. 2(3): p. 280-7.

373. Pattabiraman, G., et al., IRAK4 activity controls immune responses to intracellular bacteria Listeria monocytogenes and Mycobacterium smegmatis. J Leukoc Biol, 2018. 104(4): p. 811-820.

374. Picard, C., et al., Clinical features and outcome of patients with IRAK-4 and MyD88 deficiency. Medicine (Baltimore), 2010. 89(6): p. 403-25.

375. Brzezinska, A.A., et al., Signalling mechanisms for Toll-like receptor-activated neutrophil exocytosis: key roles for interleukin-1-receptor-associated kinase-4 and phosphatidylinositol 3-kinase but not Toll/IL-1 receptor (TIR) domain-containing adaptor inducing IFN-beta (TRIF). Immunology, 2009. 127(3): p. 386-97.

376. He, J., et al., Munc13-4 interacts with syntaxin 7 and regulates late endosomal maturation, endosomal signaling, and TLR9-initiated cellular responses. Mol Biol Cell, 2016. 27(3): p. 572-87.

377. Hajishengallis, G., Complement and periodontitis. Biochem Pharmacol, 2010. 80(12): p. 1992-2001.

378. Underhill, D.M., et al., The Toll-like receptor 2 is recruited to macrophage phagosomes and discriminates between pathogens. Nature, 1999. 401(6755): p. 811-5. 
379. Ozinsky, A., et al., The repertoire for pattern recognition of pathogens by the innate immune system is defined by cooperation between toll-like receptors. Proc Natl Acad Sci U S A, 2000. 97(25): p. 13766-71.

380. O'Connell, C.M., et al., Localization of TLR2 and MyD88 to Chlamydia trachomatis inclusions. Evidence for signaling by intracellular TLR2 during infection with an obligate intracellular pathogen. J Biol Chem, 2006. 281(3): p. 1652-9.

381. Cervantes, J.L., et al., Phagosomal signaling by Borrelia burgdorferi in human monocytes involves Toll-like receptor (TLR) 2 and TLR8 cooperativity and TLR8-mediated induction of IFN-beta. Proc Natl Acad Sci U S A, 2011. 108(9): p. 3683-8.

382. Ip, W.K., et al., Mannose-binding lectin enhances Toll-like receptors 2 and 6 signaling from the phagosome. J Exp Med, 2008. 205(1): p. 169-81.

383. Hoebe, K., et al., CD36 is a sensor of diacylglycerides. Nature, 2005. 433(7025): p. 5237.

384. Jimenez-Dalmaroni, M.J., et al., Soluble CD36 ectodomain binds negatively charged diacylglycerol ligands and acts as a co-receptor for TLR2. PLoS One, 2009. 4(10): p. e7411.

385. Tan, Y. and J.C. Kagan, Microbe-inducible trafficking pathways that control Toll-like receptor signaling. Traffic, 2017. 18(1): p. 6-17.

386. Athens, J.W., et al., Leukokinetic studies. IV. The total blood, circulating and marginal granulocyte pools and the granulocyte turnover rate in normal subjects. J Clin Invest, 1961. 40: p. 989-95.

387. Hsieh, M.M., et al., Prevalence of neutropenia in the U.S. population: age, sex, smoking status, and ethnic differences. Ann Intern Med, 2007. 146(7): p. 486-92.

388. Giammarco, S., et al., Hyperleukocytosis and leukostasis: management of a medical emergency. Expert Rev Hematol, 2017. 10(2): p. 147-154.

389. Savill, J.S., et al., Macrophage phagocytosis of aging neutrophils in inflammation. Programmed cell death in the neutrophil leads to its recognition by macrophages. J Clin Invest, 1989. 83(3): p. 865-75.

390. Savill, J.S., P.M. Henson, and C. Haslett, Phagocytosis of aged human neutrophils by macrophages is mediated by a novel "charge-sensitive" recognition mechanism. J Clin Invest, 1989. 84(5): p. 1518-27.

391. Taylor, R.C., S.P. Cullen, and S.J. Martin, Apoptosis: controlled demolition at the cellular level. Nat Rev Mol Cell Biol, 2008. 9(3): p. 231-41.

392. McCracken, J.M. and L.A. Allen, Regulation of human neutrophil apoptosis and lifespan in health and disease. J Cell Death, 2014. 7: p. 15-23.

393. Salvesen, G.S. and C.S. Duckett, IAP proteins: blocking the road to death's door. Nat Rev Mol Cell Biol, 2002. 3(6): p. 401-10.

394. Hrdinka, M. and M. Yabal, Inhibitor of apoptosis proteins in human health and disease. Genes Immun, 2019. 20(8): p. 641-650.

395. Nadeau, K.C., et al., Idiopathic neutropenia of childhood is associated with Fas/FasL expression. Clin Immunol, 2008. 129(3): p. 438-47.

396. Tait, S.W. and D.R. Green, Mitochondria and cell death: outer membrane permeabilization and beyond. Nat Rev Mol Cell Biol, 2010. 11(9): p. 621-32.

397. Green, D.R. and G. Kroemer, The pathophysiology of mitochondrial cell death. Science, 2004. 305(5684): p. 626-9.

398. Dzhagalov, I., A. St John, and Y.W. He, The antiapoptotic protein Mcl-1 is essential for the survival of neutrophils but not macrophages. Blood, 2007. 109(4): p. 1620-6.

399. Milot, E. and J.G. Filep, Regulation of neutrophil survival/apoptosis by Mcl-1. ScientificWorldJournal, 2011. 11: p. 1948-62.

400. Moulding, D.A., et al., BCL-2 family expression in human neutrophils during delayed and accelerated apoptosis. J Leukoc Biol, 2001. 70(5): p. 783-92. 
401. Kim, J.S., et al., Caspase-3 activity and expression of Bcl-2 family in human neutrophils by Helicobacter pylori water-soluble proteins. Helicobacter, 2001. 6(3): p. 207-15.

402. Leuenroth, S.J., et al., The loss of Mcl-1 expression in human polymorphonuclear leukocytes promotes apoptosis. J Leukoc Biol, 2000. 68(1): p. 158-66.

403. Moulding, D.A., et al., Mcl-1 expression in human neutrophils: regulation by cytokines and correlation with cell survival. Blood, 1998. 92(7): p. 2495-502.

404. Kato, T., et al., Cyclic AMP delays neutrophil apoptosis via stabilization of Mcl-1. FEBS Lett, 2006. 580(19): p. 4582-6.

405. Scaffidi, C., et al., Analysis of the CD95 (APO-1/Fas) death-inducing signaling complex by high-resolution two-dimensional gel electrophoresis. Methods Enzymol, 2000. 322: p. 363-73.

406. Korsmeyer, S.J., et al., Pro-apoptotic cascade activates BID, which oligomerizes BAK or $B A X$ into pores that result in the release of cytochrome c. Cell Death Differ, 2000. 7(12): p. 1166-73.

407. Schwartz, J.T., et al., Francisella tularensis inhibits the intrinsic and extrinsic pathways to delay constitutive apoptosis and prolong human neutrophil lifespan. J Immunol, 2012. 188(7): p. 3351-63.

408. Scheel-Toellner, D., et al., Early events in spontaneous neutrophil apoptosis. Biochem Soc Trans, 2004. 32(Pt3): p. 461-4.

409. Serrao, K.L., et al., Neutrophils induce apoptosis of lung epithelial cells via release of soluble Fas ligand. Am J Physiol Lung Cell Mol Physiol, 2001. 280(2): p. L298-305.

410. Jonsson, H., P. Allen, and S.L. Peng, Inflammatory arthritis requires Foxo3a to prevent Fas ligand-induced neutrophil apoptosis. Nat Med, 2005. 11(6): p. 666-71.

411. Watson, R.W., et al., Neutrophils undergo apoptosis following ingestion of Escherichia coli. J Immunol, 1996. 156(10): p. 3986-92.

412. Kobayashi, S.D., et al., Gene expression profiling provides insight into the pathophysiology of chronic granulomatous disease. J Immunol, 2004. 172(1): p. 636-43.

413. Zhang, B., et al., Elucidation of molecular events leading to neutrophil apoptosis following phagocytosis: cross-talk between caspase 8, reactive oxygen species, and MAPK/ERK activation. J Biol Chem, 2003. 278(31): p. 28443-54.

414. Egners, A., M. Erdem, and T. Cramer, The Response of Macrophages and Neutrophils to Hypoxia in the Context of Cancer and Other Inflammatory Diseases. Mediators Inflamm, 2016. 2016: p. 2053646.

415. Hannah, S., et al., Hypoxia prolongs neutrophil survival in vitro. FEBS Lett, 1995. 372(23): p. 233-7.

416. Colotta, F., et al., Modulation of granulocyte survival and programmed cell death by cytokines and bacterial products. Blood, 1992. 80(8): p. 2012-20.

417. Daffern, P.J., M.A. Jagels, and T.E. Hugli, Multiple epithelial cell-derived factors enhance neutrophil survival. Regulation by glucocorticoids and tumor necrosis factor-alpha. Am J Respir Cell Mol Biol, 1999. 21(2): p. 259-67.

418. Kotone-Miyahara, Y., et al., Short-term delay of Fas-stimulated apoptosis by GM-CSF as a result of temporary suppression of FADD recruitment in neutrophils: evidence implicating phosphatidylinositol 3-kinase and MEK1-ERK1/2 pathways downstream of classical protein kinase C. J Leukoc Biol, 2004. 76(5): p. 1047-56.

419. Lee, A., M.K. Whyte, and C. Haslett, Inhibition of apoptosis and prolongation of neutrophil functional longevity by inflammatory mediators. J Leukoc Biol, 1993. 54(4): p. 283-8.

420. Perianayagam, M.C., et al., C5a delays apoptosis of human neutrophils via an extracellular signal-regulated kinase and Bad-mediated signalling pathway. Eur J Clin Invest, 2004. 34(1): p. 50-6. 
421. DeLeo, F.R., Modulation of phagocyte apoptosis by bacterial pathogens. Apoptosis, 2004. 9(4): p. 399-413.

422. Kobayashi, S.D., N. Malachowa, and F.R. DeLeo, Influence of Microbes on Neutrophil Life and Death. Front Cell Infect Microbiol, 2017. 7: p. 159.

423. Murphy, M.P. and E. Caraher, Mcl-1 is vital for neutrophil survival. Immunol Res, 2015. 62(2): p. 225-33.

424. Galluzzi, L., et al., Guidelines for the use and interpretation of assays for monitoring cell death in higher eukaryotes. Cell Death Differ, 2009. 16(8): p. 1093-107.

425. Mayadas, T.N. and X. Cullere, Neutrophil beta2 integrins: moderators of life or death decisions. Trends Immunol, 2005. 26(7): p. 388-95.

426. Mcllwain, D.R., T. Berger, and T.W. Mak, Caspase functions in cell death and disease. Cold Spring Harb Perspect Biol, 2013. 5(4): p. a008656.

427. Hochreiter-Hufford, A. and K.S. Ravichandran, Clearing the dead: apoptotic cell sensing, recognition, engulfment, and digestion. Cold Spring Harb Perspect Biol, 2013. 5(1): p. a008748.

428. Crawford, J.M., J.M. Wilton, and P. Richardson, Neutrophils die in the gingival crevice, periodontal pocket, and oral cavity by necrosis and not apoptosis. J Periodontol, 2000. 71(7): p. 1121-9.

429. Gardai, S.J., et al., Oxidants inhibit ERK/MAPK and prevent its ability to delay neutrophil apoptosis downstream of mitochondrial changes and at the level of XIAP. J Biol Chem, 2004. 279(43): p. 44695-703.

430. Simons, M.P., et al., Neisseria gonorrhoeae delays the onset of apoptosis in polymorphonuclear leukocytes. Cell Microbiol, 2006. 8(11): p. 1780-90.

431. van Zandbergen, G., et al., Chlamydia pneumoniae multiply in neutrophil granulocytes and delay their spontaneous apoptosis. J Immunol, 2004. 172(3): p. 1768-76.

432. Acorci, M.J., et al., Inhibition of human neutrophil apoptosis by Paracoccidioides brasiliensis: role of interleukin-8. Scand J Immunol, 2009. 69(2): p. 73-9.

433. Chen, S.M., et al., Identification of a granulocytotropic Ehrlichia species as the etiologic agent of human disease. J Clin Microbiol, 1994. 32(3): p. 589-95.

434. Dumler, J.S., et al., Reorganization of genera in the families Rickettsiaceae and Anaplasmataceae in the order Rickettsiales: unification of some species of Ehrlichia with Anaplasma, Cowdria with Ehrlichia and Ehrlichia with Neorickettsia, descriptions of six new species combinations and designation of Ehrlichia equi and 'HGE agent' as subjective synonyms of Ehrlichia phagocytophila. Int J Syst Evol Microbiol, 2001. 51(Pt 6): p. 21452165.

435. Yoshiie, K., et al., Intracellular infection by the human granulocytic ehrlichiosis agent inhibits human neutrophil apoptosis. Infect Immun, 2000. 68(3): p. 1125-33.

436. Scaife, H., et al., Anaplasma phagocytophilum reduces neutrophil apoptosis in vivo. Infect Immun, 2003. 71(4): p. 1995-2001.

437. Klein, M.B., et al., The agent of human granulocytic ehrlichiosis induces the production of myelosuppressing chemokines without induction of proinflammatory cytokines. J Infect Dis, 2000. 182(1): p. 200-5.

438. Kim, H.Y. and Y. Rikihisa, Expression of interleukin-1beta, tumor necrosis factor alpha, and interleukin-6 in human peripheral blood leukocytes exposed to human granulocytic ehrlichiosis agent or recombinant major surface protein P44. Infect Immun, 2000. 68(6): p. 3394-402.

439. Mott, J. and Y. Rikihisa, Human granulocytic ehrlichiosis agent inhibits superoxide anion generation by human neutrophils. Infect Immun, 2000. 68(12): p. 6697-703.

440. Wang, T., et al., Superoxide anion production during Anaplasma phagocytophila infection. J Infect Dis, 2002. 186(2): p. 274-80. 
441. de la Fuente, J., et al., Anaplasma phagocytophilum Uses Common Strategies for Infection of Ticks and Vertebrate Hosts. Trends Microbiol, 2016. 24(3): p. 173-180.

442. Morioka, S., C. Maueroder, and K.S. Ravichandran, Living on the Edge: Efferocytosis at the Interface of Homeostasis and Pathology. Immunity, 2019. 50(5): p. 1149-1162.

443. Doran, A.C., A. Yurdagul, Jr., and I. Tabas, Efferocytosis in health and disease. Nat Rev Immunol, 2019.

444. Headland, S.E. and L.V. Norling, The resolution of inflammation: Principles and challenges. Semin Immunol, 2015. 27(3): p. 149-60.

445. Morioka, S., et al., Efferocytosis induces a novel SLC program to promote glucose uptake and lactate release. Nature, 2018. 563(7733): p. 714-718.

446. Tajbakhsh, A., et al., The role of nuclear factors as "Find-Me"/alarmin signals and immunostimulation in defective efferocytosis and related disorders. Int Immunopharmacol, 2020. 80: p. 106134.

447. Bagaitkar, J., et al., NADPH oxidase activation regulates apoptotic neutrophil clearance by murine macrophages. Blood, 2018. 131(21): p. 2367-2378.

448. Zeng, M.Y., et al., An efferocytosis-induced, IL-4-dependent macrophage-iNKT cell circuit suppresses sterile inflammation and is defective in murine CGD. Blood, 2013. 121(17): p. 3473-83.

449. Guzik, K., et al., A new insight into phagocytosis of apoptotic cells: proteolytic enzymes divert the recognition and clearance of polymorphonuclear leukocytes by macrophages. Cell Death Differ, 2007. 14(1): p. 171-82.

450. Guzik, K. and J. Potempa, Friendly fire against neutrophils: proteolytic enzymes confuse the recognition of apoptotic cells by macrophages. Biochimie, 2008. 90(2): p. 405-15.

451. Lodge, K.M., et al., The Impact of Hypoxia on Neutrophil Degranulation and Consequences for the Host. Int J Mol Sci, 2020. 21(4).

452. Campbell, E.L., et al., Transmigrating neutrophils shape the mucosal microenvironment through localized oxygen depletion to influence resolution of inflammation. Immunity, 2014. 40(1): p. 66-77.

453. Loesche, W.J., et al., Relationship between oxygen tension and subgingival bacterial flora in untreated human periodontal pockets. Infect Immun, 1983. 42(2): p. 659-67.

454. Barclay, A.N. and T.K. Van den Berg, The interaction between signal regulatory protein alpha (SIRPalpha) and CD47: structure, function, and therapeutic target. Annu Rev Immunol, 2014. 32: p. 25-50.

455. Brown, S., et al., Apoptosis disables CD31-mediated cell detachment from phagocytes promoting binding and engulfment. Nature, 2002. 418(6894): p. 200-3.

456. Korkmaz, B., et al., Neutrophil elastase, proteinase 3, and cathepsin $G$ as therapeutic targets in human diseases. Pharmacol Rev, 2010. 62(4): p. 726-59.

457. Vandivier, R.W., et al., Elastase-mediated phosphatidylserine receptor cleavage impairs apoptotic cell clearance in cystic fibrosis and bronchiectasis. J Clin Invest, 2002. 109(5): p. 661-70.

458. McGovern, N.N., et al., Hypoxia selectively inhibits respiratory burst activity and killing of Staphylococcus aureus in human neutrophils. J Immunol, 2011. 186(1): p. 453-463.

459. Martin, C.J., et al., Efferocytosis is an innate antibacterial mechanism. Cell Host Microbe, 2012. 12(3): p. 289-300.

460. Dallenga, T., et al., M. tuberculosis-Induced Necrosis of Infected Neutrophils Promotes Bacterial Growth Following Phagocytosis by Macrophages. Cell Host Microbe, 2017. 22(4): p. 519-530 e3.

461. Blander, J.M., The comings and goings of MHC class I molecules herald a new dawn in cross-presentation. Immunol Rev, 2016. 272(1): p. 65-79. 


\section{CURRICULUM VITAE}

Irina Miralda Molina

Louisville, KY | (502)645-8355 | irinamiralda@gmail.com

\section{$\underline{\text { Education }}$}

Doctorate | Expected April 2020 | University of Louisville

- Microbiology \& Immunology

- Dissertation Topic: Emerging Oral Bacteria and Innate Immunity

Masters | April 2017 | University of Louisville

- Microbiology \& Immunology

- GPA: 3.747

Bachelor's in science | May 2014 | University of Louisville

- Microbiology \& Immunology

- GPA: 3.791

\section{Employment \& Research experience}

2015-2020 Research Fellow, Departments of Medicine and Microbiology and Immunology, School of Medicine, University of Louisville, KY.

2014-2015 Graduate student researcher, Department of Medicine, School of Medicine, University of Louisville, KY.

2014-2015 Neonatal Intensive Care Unit Lactation Technician

$2014 \quad$ Undergraduate Honor Thesis

2012-2014 Undergraduate Researcher, Departments of Medicine and Microbiology and Immunology, School of Medicine, University of Louisville, KY.

2013-2014 Emergency Department Medical Scribe

2012 Internship through Summer Research Opportunity Program with Dr. Silvia Uriarte.

\section{Teaching experience}


Fall 2019 "In vitro analysis of Immune Cell Functions" Lecturer in Microbiology \& Immunology Methods course for Graduate Students at University of Louisville.

2013-2014 STEM Undergraduate Teaching Assistant

\section{$\underline{\text { Mentoring experience }}$}

2018-2020 Max Rogers, undergraduate student. Project title: "Filifactor alocis: resilience and manipulation against neutrophil effector functions in a dysbiotic oral environment." Defending Undergraduate Honors Thesis Spring 2020.

Fall 2019 Tyrell Jamison, graduate student. Project title: "Differential MAPK signaling in human neutrophils by viable and heat-killed Filifactor alocis."

Summer 2019 Bertha Aguazul, undergraduate student. Project title: "Reactive Oxygen Species generation against the emerging oral pathogen, Selenomonas sputigena."

Spring 2019 Alexa Smothers, high school student. Project title: "Effect of Cinnamaldehyde on Streptococcus gordonii." $3^{\text {rd }}$ Place Winner in Microbiology Category of Louisville Regional Science \& Engineering Fair

Summer 2017 Thomas Case, undergraduate student. Project title: "SOCS 1 Role in Manipulation of Neutrophil Signaling by Oral Pathogen, Filifactor alocis."

\section{$\underline{\text { Service \& Volunteering }}$}

2019 \& 2020 Science Ambassador for Louisville Regional Science \& Engineering Fair 2019 Organized activity titled, "A Closer Look: Bacteria \& Blood Cells" for the Girls Rule! STEM-H Summit for girls in grades 3-12 on behalf of Society for Advancement of Chicanos, Latinos and Native Americans in Science (SACNAS) Chapter.

2017-Present Founding member and current vice-president of the first Society for Advancement of Chicanos, Latinos and Native Americans in Science (SACNAS) chapter in the state of Kentucky.

2018-2019 Volunteer \& member of Organization Assisting and Serving International Students (OASIS) 
2017-2018 President for University of Louisville Microbiology \& Immunology Student Organization (MISO)

2016-2019 Junior Editor for the Society for Leukocyte Biology newsletter.

\section{Awards \& Honors}

2020 Recipient of the John Richard Binford Memorial Award that recognizes a doctoral degree recipient who excels in scholarship and has contributed to other areas within the discipline such as leadership, teaching, or service.

2019 Student travel award winner for annual meeting of the Society for Leukocyte Biology: "Tissue Specific Immunity: Translating our Discoveries."

2019 Invited participant for St. Jude Children Hospital's National Graduate Student Symposium

2018 Student travel award winner for annual meeting of the Society for Leukocyte Biology: "Myeloid Cells: Development, Environment, and Inflammation."

2018 Recognized for Excellence in Graduate Diversity by the University of Louisville School of Interdisciplinary and Graduate Studies.

2017 Completed the School on Interdisciplinary and Graduate Studies Publishing Academy

2016 John Wallace Scholarship for 2016 Autumn Immunology Conference.

2016 Travel Scholarship for 2016 Society for Advancement of Chicanos, Latinos and Native Americans in Science (SACNAS)- The national diversity in STEM Conference.

2015 Student travel Award Winner for 48th annual meeting of the Society for Leukocyte Biology: Immunity in Health and Disease

2015 Graduate Student Fellowship from Integrated Program in Biomedical Sciences (IPIBS)

2014 Student travel Award Winner for 47th annual meeting of the Society for Leukocyte Biology: Development of Innate Immunity

2014 David G. Smith Memorial Award winner for outstanding research achievements as an undergraduate

2014 N.V LeBre Award Winner for the most significant work in some aspect of Biology by an undergraduate

2013 Student travel award recipient for 46th annual meeting of the Society for Leukocyte Biology: Regulators of Innate Cell Plasticity

\section{Presentations (First Author)}

2019 Society of Leukocyte Biology $52^{\text {nd }}$ annual meeting's Poster Talk session. Oral Presentation: "The TLR2 Tango: Contributions of human neutrophils and the putative oral pathogen Filifactor alocis to periodontitis" 
2019 Poster presenter at St. Jude's National Graduate Student Symposium.

"Signaling tug-of-war between Filifactor alocis and Neutrophils."

2018 Poster presenter in the annual meeting of the Society for Leukocyte Biology. "Filifactor alocis Activates a MyD88-independent, TLR-NFאB Signaling Axis in Neutrophils."

2016 Autumn Immunology Conference Oral Presentation: "Role of complement in neutrophil responses against environmental mycobacteria" and poster presentation.

2015 Society of Leukocyte Biology $48^{\text {th }}$ annual meeting's Poster Talk session. Oral Presentation: "Gelatinase Granules not generation of reactive oxygen species, participates in human neutrophil killing of environmental mycobacteria."

2015 Poster presenter in Research! Louisville.

2014 Society of Leukocyte Biology $47^{\text {th }}$ annual meeting's concurrent session "The Human Microbiome in Health and Disease." Oral presentation:

Phosphoinositol lipoarabinomannan involvement in Mycobacterium smegmatis modulation of neutrophil functions.

2014 Poster presenter in Research! Louisville.

2014 Poster presenter at Undergraduate Research and Community Engagement Symposium

2013 Poster presenter in the 46th annual meeting of the Society for Leukocyte Biology: Regulators of Innate Cell Plasticity

2012 Poster presentation at conclusion of SROP in Louisville, KY.

\section{$\underline{\text { Professional memberships }}$}

2016 Member, Society for Advancement of Chicanos, Latinos and Native Americans in Science (SACNAS)

2013 Member, Society of Leukocyte Biology (SLB)

\section{$\underline{\text { Peer-reviewed Publications }}$}

Miralda I, Vashishta A., Rogers MR, Rouchka EC, Li X, Waigel S, Lamont RJ, Uriarte SM (2020). Whole transcriptome analysis reveals that Filifactor alocis modulates TNF $\alpha$-stimulated MAPK activation in human neutrophils. Front. Immunol. Accepted.

Miralda I, Klaes CK, Graham JE, and Uriarte SM (2020). Human Neutrophil Granule Exocytosis in Response to Mycobacterium smegmatis. Pathogens 9(2). doi: 10.3390/pathogens9020123. 
Vashishta A, Jimenez-Flores E, Klaes CK, Tian S, Miralda I, Lamont RJ, Uriarte SM, (2019). Putative Periodontal Pathogens, Filifactor alocis and Peptoanaerobacter Stomatis, Induce Differential Cytokine and Chemokine Production by Human Neutrophils. Pathogens. doi:10.3390/pathogens8020059. PMID: 31052371

Zeng MY, Miralda I, Armstrong CL, Uriarte SM, Bagaitkar J. (2019). Review: The diverse roles of NADPH oxidase and derivative ROS in modulating neutrophil effector functions and host inflammation. Mol Oral Microbiol. Journal of Molecular Oral Microbiology. doi: 10.1111/omi.12252. PMID 30632295

Bagaitkar J, Huang J, Zeng MY, Pech NK, Monlish DA, Perez-Zapata LJ, Miralda I, Schuettpelz LG, Dinauer MC., (2018). NADPH oxidase activation regulates apoptotic neutrophil clearance by murine macrophages. Blood. doi: 10.1182/blood-2017-09-809004. PMID: 29618478

Edmisson JS, Tian S, Armstrong CL, Vashishta A, Klaes CK, Miralda I, Jimenez-Flores E, Le J, Wang Q, Lamont RJ, Uriarte SM., (2018). Filifactor alocis modulates human neutrophil antimicrobial functional responses. Cell Microbiol. doi: 10.1111/cmi.12829. PMID: 29377528

Miralda, I., Uriarte, S.M., McLeish, K.R., (2017). Review: Multiple Phenotypic Changes Define Neutrophil Priming. Front. Cell. Infect. Microbiol. doi:10.3389/fcimb.2017.00217. PMID: 28611952

Castor, D.J., Powell, D.W., Miralda, I., Ward, R.A., McLeish, K.R., (2017). Review: Reexamining Neutrophil Participation in Glomerulonephritis. J. Am. Soc. Nephrol. doi:10.1681/ASN.2016121271. PMID: 28620081

Armstrong, C.L., Miralda, I., Neff, A.C., Tian, S., Vashishta, A., Perez, L., et al. (2016). Filifactor alocis Promotes Neutrophil Degranulation and Chemotactic Activity. Infect Immun 84(12), 3423-3433. doi: 10.1128/IAI.00496-16. PMID: 27647870

\section{$\underline{\text { Book Chapters }}$}

Miralda I, Vashishta A, and Uriarte SM.: Neutrophil interaction with emerging oral pathogens: A novel view of the disease paradigm. In: Oral Mucosal Immunity and Microbiome, Eds. George Belibasakis and George Hajishengallis. Springer (2019), In press.

\section{$\underline{\text { Research Support }}$}

2017 -2019 R01 DE024509-S1 Research Supplement to Promote Diversity in HealthRelated Research (NIH-NIDCR)

2014-2016 Graduate Student Fellowship from Integrated Program in Biomedical Sciences (IPIBS) 